\title{
MODOS NÃO-LINEARES DE VIBRAÇÃO E CONTROLE ATIVO DE RISERS
}

Tese de Doutorado

Área de Concentração:

Engenharia de Estruturas

Orientador:

Prof. Dr. Carlos Eduardo Nigro Mazzilli

Tese apresentada à Escola Politécnica

da Universidade de São Paulo para obtenção

do Título de Doutor em Engenharia Civil.

São Paulo 
Este exemplar foi revisado e alterado em relação à versão original, sob responsabilidade única do autor e com a anuência de seu orientador.

São Paulo, 26 de março de 2009.

Assinatura do autor

Assinatura do orientador

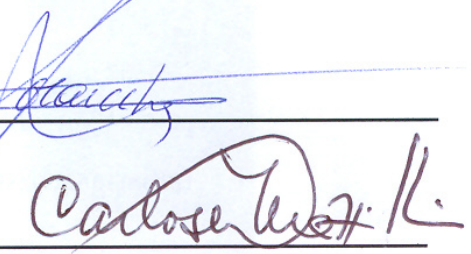

FICHA CATALOGRÁFICA

Sanches, César Tarabay

Modos não-lineares de vibração e controle ativo de risers I

C.T. Sanches. -- ed.rev. -- São Paulo, 2009. $170 \mathrm{p}$.

Tese (Doutorado) - Escola Politécnica da Universidade de São Paulo. Departamento de Engenharia de Estruturas e Geotéc-nica.

1. Engenharia de estruturas e fundações 2. Engenharia naval e oceânica 3. Engenharia mecânica I. Universidade de São Paulo. Escola Politécnica. Departamento de Engenharia de Estrutu ras e Geotécnica II. t. 
"A prayer for the wild at heart, kept in cages." Tennessee Williams

"Mas, indo bem fundo, chego muito pensativa à conclusão de que não existe nada mais difícil que entregar-se totalmente. Essa dificuldade é uma das dores humanas." Clarice Lispector. 
À minha grande e eterna paixão, Ana. Aos meus eternos amores:

Pai, Mãe, Caio, Vó, Kauã e Branquinha. 


\section{Agradecimentos}

Antes de tudo, gostaria de dizer muito obrigado ao meu orientador, Professor Carlos Eduardo Nigro Mazzilli. Sem ele, este texto não estaria aqui. Foram muitos altos e baixos, típicos de uma jornada ao desconhecido. Imagino que não tenha sido nada fácil para ele a empreitada de me orientar. Agradeço muito todo o esforço e paciência dele nesta árdua tarefa.

Agradeço de forma especial à minha família: Pai, Mãe, Caio e ao meu, recém chegado e muito amado, sobrinho e afilhado, Kauã. Eles são parte de minha história e, portanto, parte de mim mesmo. Agradeço ao meu Pai pelo exemplo de perseverança e força. Mesmo diante das situações mais adversas, ele nunca fraquejou. Espero que minha Mãe esteja vendo esta minha realização e que isto seja motivo de alegria para ela. Agradeço à minha Avó, que tão pacientemente me ensinou minhas primeiras lições. Não posso deixar de agradecer à Branquinha por ter me ensinado a ser mais humano. Obrigado à Ilda pelo calor humano. Agradeço ao Zé e à Beta por terem sempre me tratado como filho e pelos deliciosos almoços de Domingo. Ao Pedro, obrigado por ter vindo de Lorena para prestigiar minha defesa. Ao Giuseppe e à Nayse, agradeço por terem me recebido de braços abertos. Agradeço à Vó Nayse e à Mainha por me tratarem como neto e pelo exemplo de força que elas são.

Ao meu velho amigo Campello, sou grato por ter caminhado comigo, lado a lado, durante todo o tempo. Agradeço ao meu grande amigo Odulpho pela ajuda com os multi-modos e, principalmente, pelo convívio e amizade de tantos anos. Agradeço ao meu caro amigo lisboeta, Carlos Tiago, que, além de me receber e apresentar Lisboa, sempre me incentivou a concluir o doutorado. Agradeço aos meus grandes amigos de Aberdeen, “The Cheap Guys": Paolo, Christian, Ralf e Mario. Sem eles não teria sido possível sobreviver ao intimidador e gélido cinza de Aberdeen. Agradeço ao meu amigo e parceiro de pesquisa, Marko Keber, pelo apoio durante os trabalhos e pelas aulas sobre a Eslovênia. Um muito obrigado, também, ao meu grande amigo Januário pelo companheirismo e apoio durante todos esses anos. Ao Márcio, agradeço a ajuda na longa e intrigante caminhada ao autoconhecimento. 
Magali, muito obrigado por ter me auxiliado a atravessar os vales. Obrigado aos meus caros amigos Henrique e Estela que resistiram bravamente às longas horas da defesa e às cervejas que se seguiram. Agradeço ao Cristiano Schmidt pela amizade e suporte aos trabalhos desenvolvidos no laboratório.

Agradeço à CAPES pelo apoio a este trabalho. Ao Departamento de Estruturas e Fundações da EPUSP, agradeço pelo incentivo e suporte. Esta pesquisa foi, em sua maior parte, elaborada no LMC (Laboratório de Mecânica Computacional) da EPUSP, que proporcionou os recursos materiais necessários para a realização dos trabalhos. Além disso, o LMC teve - e tem - papel fundamental na criação de um ambiente cientificamente instigante, pois permite o convívio de colegas pesquisadores, estimulando a discussão científica e o florescimento de novas idéias.

Agradeço ao Professor Raúl González pelo exemplo de espírito científico e por ter me auxiliado e apoiado durante os desenvolvimentos de sistemas de controle utilizando MEF. Um muito obrigado aos Professores Celso Pesce e Clóvis Martins pelos esclarecimentos sobre o comportamento dinâmico de risers e pelo apoio dado a esta pesquisa especialmente enquanto estive em Lisboa para o ISOPE.

Agradeço à Universidade de Aberdeen por ter me recebido em suas dependências e proporcionado um ambiente agradabilíssimo para o desenvolvimento dos trabalhos científicos. Boa parte deste trabalho foi desenvolvida no Fraser Noble Building e no Hub. Agradeço de forma especial ao Professor Marian Wiercigroch, que, além de me co-orientar enquanto estava em Aberdeen, sempre apoiou e incentivou este trabalho. Sou muito grato, também, à Royal Society pelo apoio e incentivo a esta pesquisa, especialmente, com relação aos desenvolvimentos realizados em Aberdeen.

Agradeço a todos que, conscientemente ou não, fizeram parte desta aventura. 


\section{Agradecimento Especial}

Estas linhas se fazem poucas para expressar minha gratidão para com esta pessoa. Sem ela, nada faria sentido. O sol a pino no Leblon seria gélido. A aconchegante e viva Amsterdam seria fria e distante. A Torre Eiffel apenas um amontoado de metal. O apaixonante e alaranjado pôr-do-sol em Aberdeen seria cinza e sem vida.

Ela me apoiou nos momentos mais difícieis: nas frustrações, nas perdas, nas dores... me acompanhou nas maiores alegrias e realizações... também dividimos o simples cotidiano... quebrando a cabeça para fazer um novo prato ou dividindo a pipoca no cinema...

Ela, mais do que qualquer outra pessoa, sabe como foi absorvente e intensa a elaboração deste trabalho. Ela sabe das angústias e ansiedades... Ela me apoiou e teve uma enorme força, que não sei de onde ela consegue ... Ela, mais do que eu mesmo, acreditava...

Por isso tudo, e muito mais, dedico este trabalho e agradeço imensamente à minha sempre amada Ana. 
CÉSAR TARABAY SANCHES

MODOS NÃO-LINEARES DE VIBRAÇÃO E CONTROLE ATIVO DE RISERS

Tese de Doutorado

Área de Concentração:

Engenharia de Estruturas

Orientador:

Prof. Dr. Carlos Eduardo Nigro Mazzilli

São Paulo

2009 


\section{Sumário}

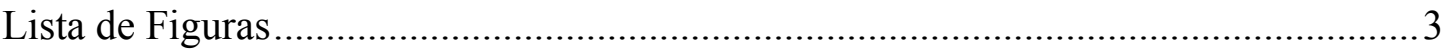

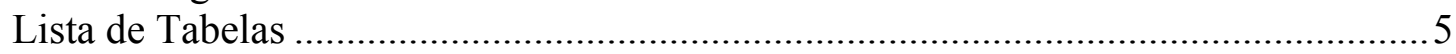

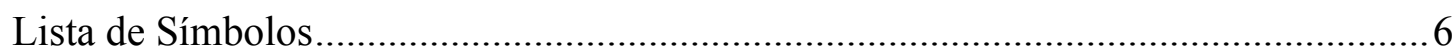

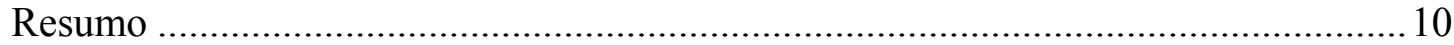

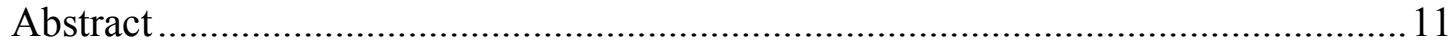

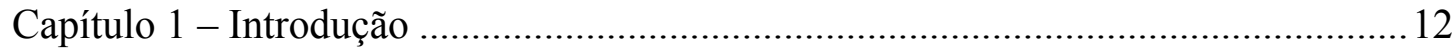

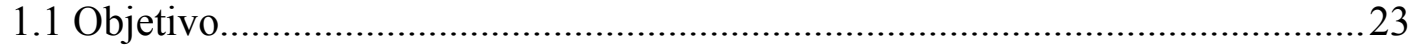

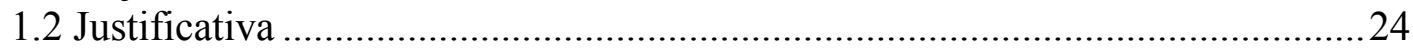

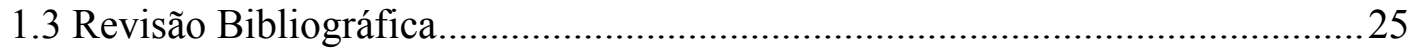

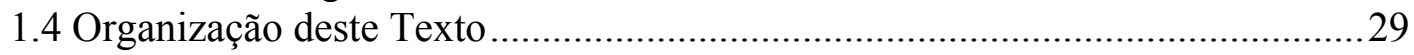

Capítulo 2 - Modos Não-Lineares de Vibração ......................................................... 31

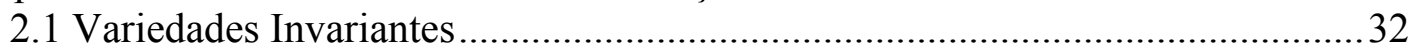

2.1.1 Variedades Invariantes em Sistemas Lineares........................................... 32

2.1.2 Variedades Invariantes em Sistemas Não-Lineares ....................................39

2.2 Sistema Discretizado pelo Método dos Elementos Finitos................................48

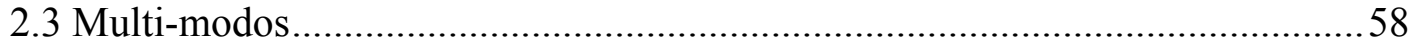

Capítulo 3 - Risers Retos Sujeitos a Carregamentos Axiais .......................................63

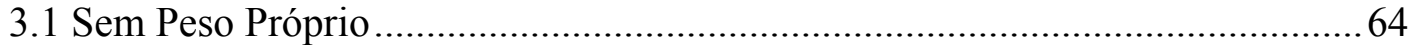

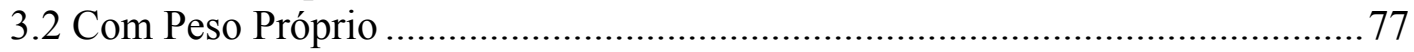

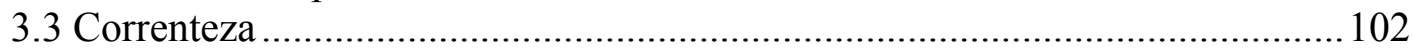

3.3.1 Vibração Induzida por Vórtices - Riser Reto ..........................................109

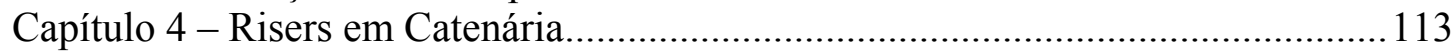

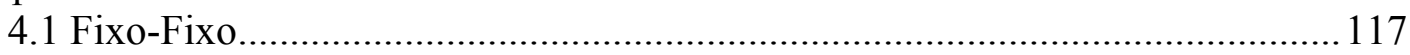

4.1.1 Vibração Induzida por Vórtices - Riser em Catenária ............................ 121

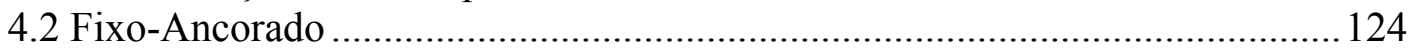

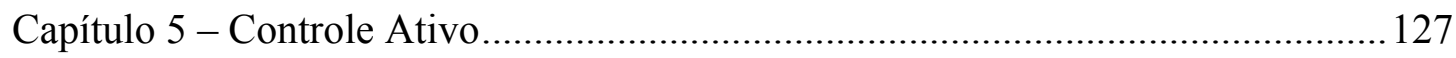

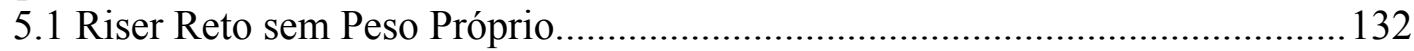

5.2 Riser Reto com Peso Próprio ................................................................................... 138

5.3 Riser em Catenária Com Extremos Fixos, VIV e Controle .............................. 143

5.4 Controle Ótimo - Regulador Quadrático Linear ............................................. 146

5.4.1 "Riser" Reto sem Peso Próprio .................................................................. 149

5.4.2 "Riser” Reto com Peso Próprio................................................................ 153

5.4.3 Riser Reto com VIV e Controle.............................................................. 158

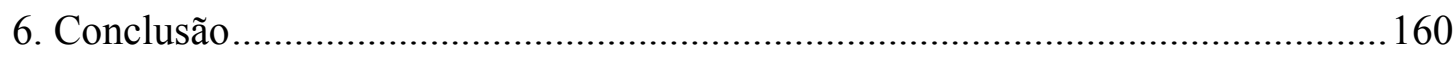

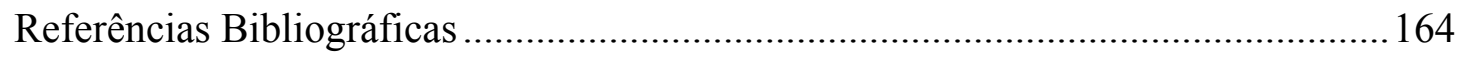




\section{Lista de Figuras}

1.1 - Campo de exploração em águas profundas com Riser Towers........................... 15

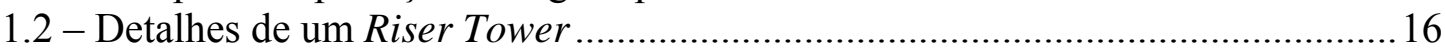

1.3 - Campo de exploração em águas profundas com SCR's .................................. 17

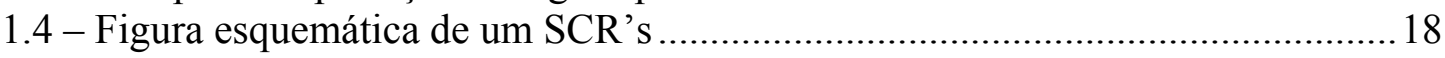

1.5 - Comparação de custo entre Riser Tower e SCR ...............................................19

1.6 - Espectro de energia de onda característica para mar bi-modal..........................21

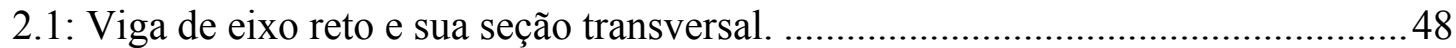

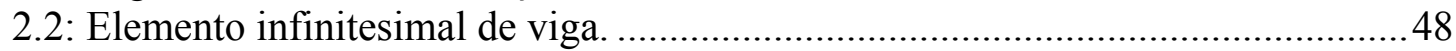

2.3: Elemento finito de viga com seis graus de liberdade........................................51

3.1 - Riser reto esquemático; Modelo de Bernoulli-Euler ............................................64

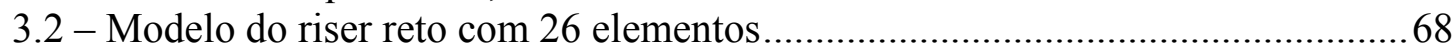

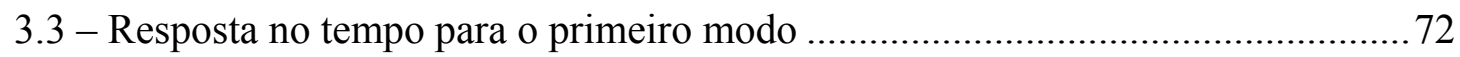

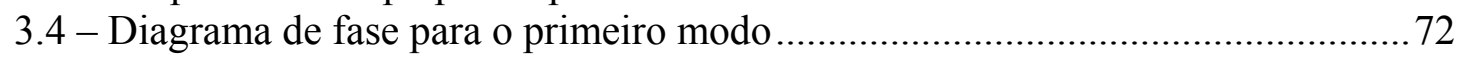

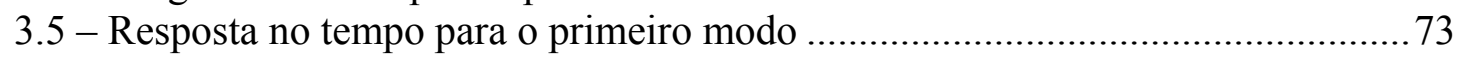

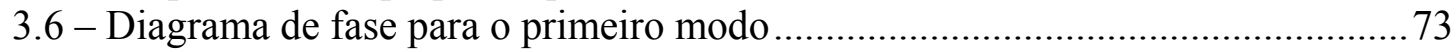

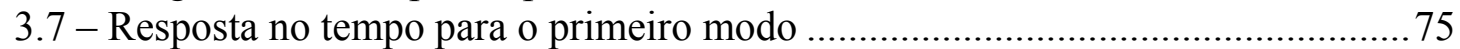

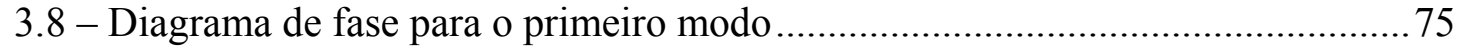

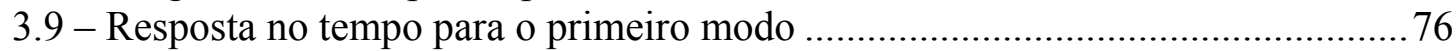

3.10 - Diagrama de fase para o primeiro modo ............................................................. 76

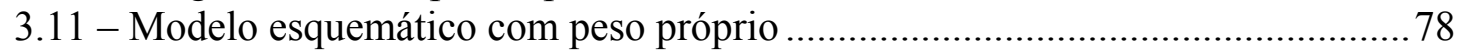

3.12 - Diagrama de fase adimensional para a resposta via MEF.............................. 86

3.13 - Resposta no tempo para o primeiro modo .................................................... 88

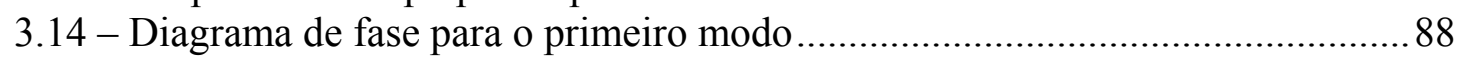

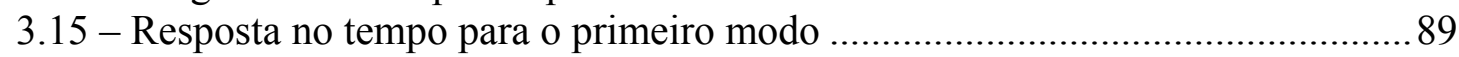

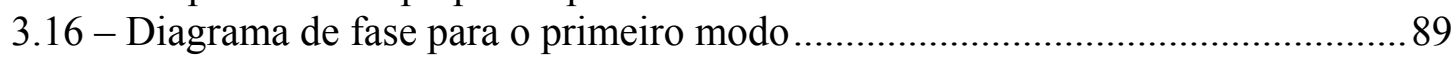

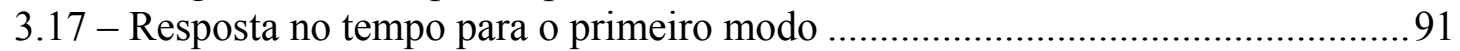

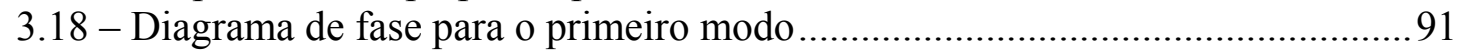

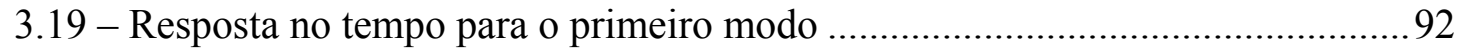

3.20 - Diagrama de fase para o primeiro modo....................................................... 92

3.21 - Forma modal do primeiro modo de vibração normal não-linear.......................93

3.22 - Diagramas de fase para o multi-modo não-linear .......................................... 99

3.23 - Diagramas de fase para as interações multi-modais ..................................... 100

3.24 - Fotografias do multi-modo (primeiro e terceiro modos) ................................ 101

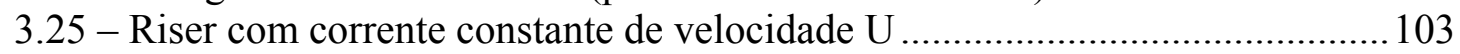

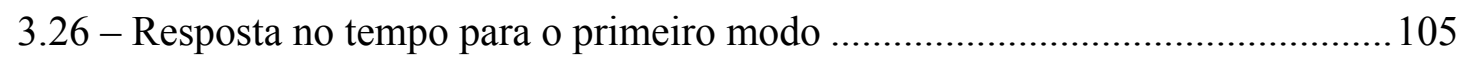

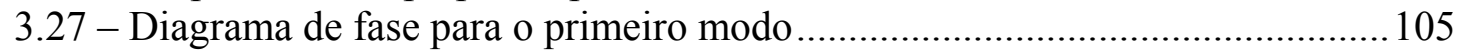

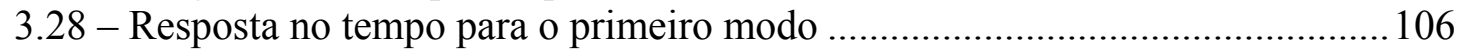

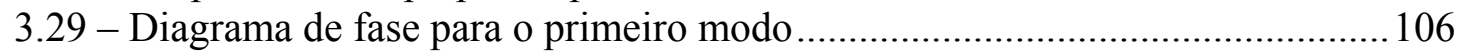

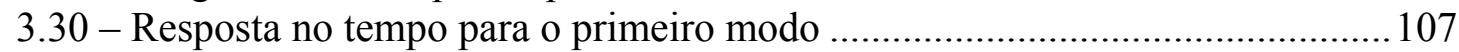

3.31 - Diagrama de fase para o primeiro modo......................................................... 107

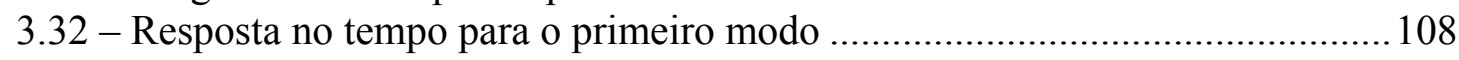

3.33 - Diagrama de fase para o primeiro modo...................................................... 108

3.34 - St e Cd para um corpo cilindríco; esteira de Von Kármán ............................. 109

3.35 - Resposta no tempo para VIV, riser reto ...................................................... 111

3.36 - Diagrama de fase para VIV, riser reto ....................................................... 111 
4.1 - Modelo esquemático de riser em catenária .................................................. 114

4.2 - Modelo de elementos finitos com numeração dos graus de liberdade.............. 114

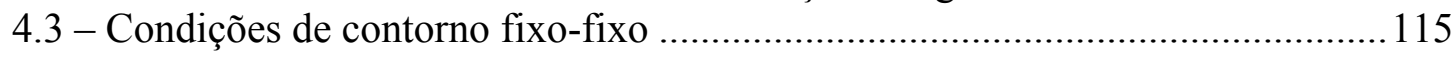

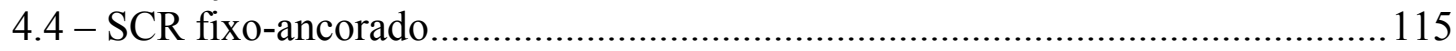

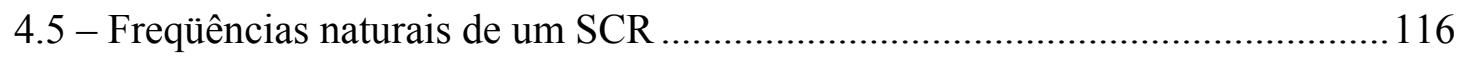

4.6 - Forma modal do primeiro modo .................................................................. 117

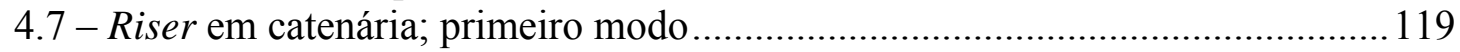

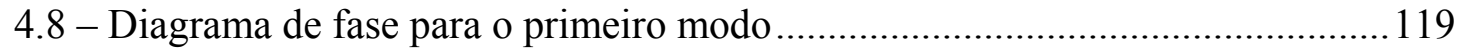

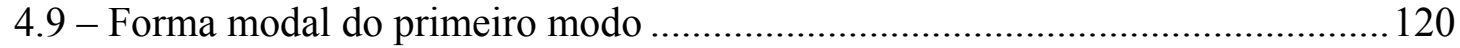

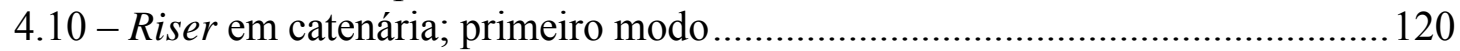

4.11 - Diagrama de fase para o primeiro modo ...................................................... 121

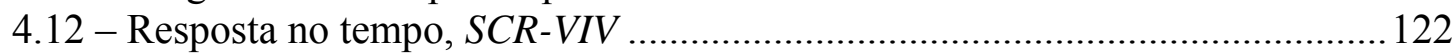

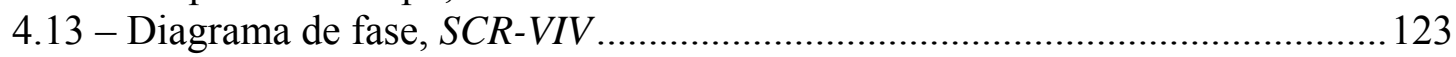

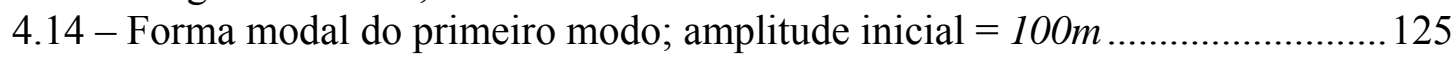

4.15 - Riser em catenária; primeiro modo; amplitude inicial de $100 \mathrm{~m}$.................... 125

4.16 - Diagrama de fase para o primeiro modo; amplitude inicial de $100 \mathrm{~m}$............. 126

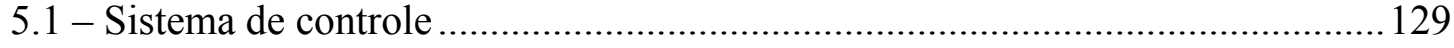

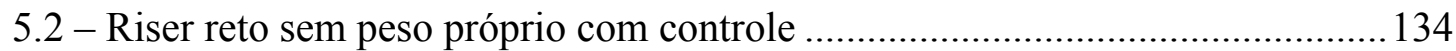

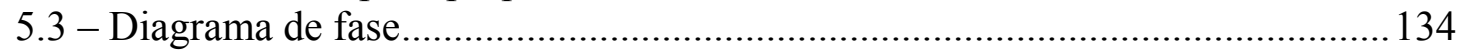

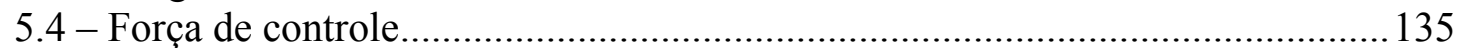

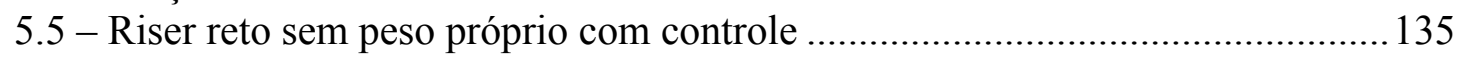

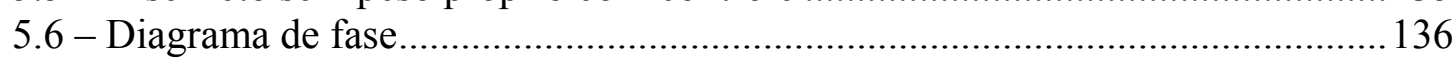

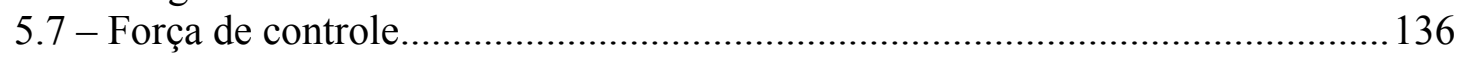

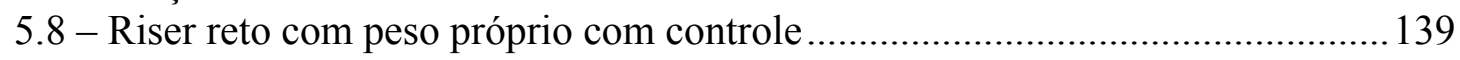

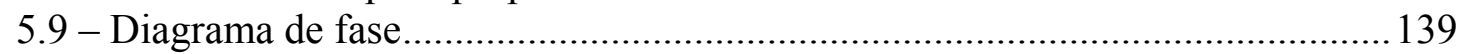

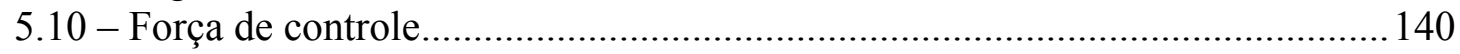

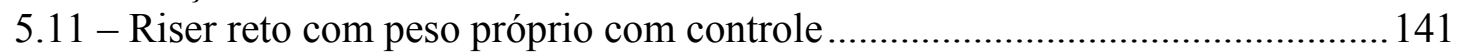

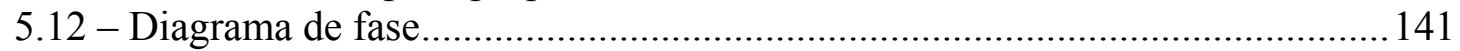

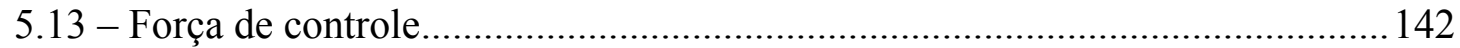

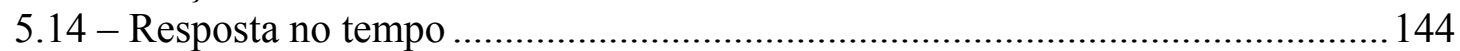

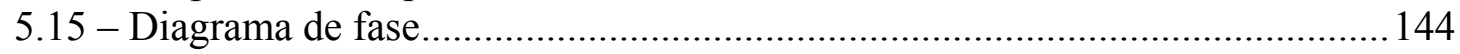

5.16 - Riser reto sem peso próprio com controle ótimo ......................................... 150

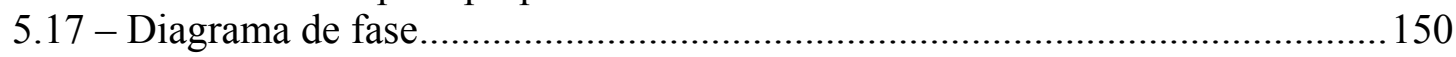

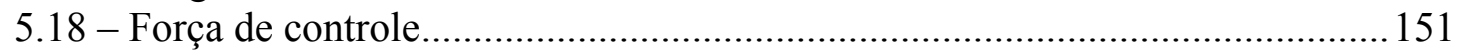

5.19 - Riser" reto sem peso próprio com controle ótimo ….................................... 152

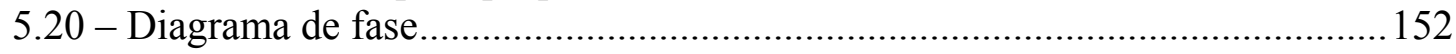

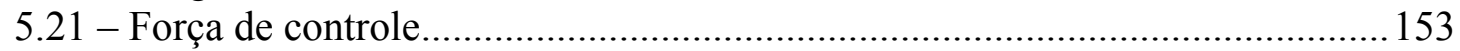

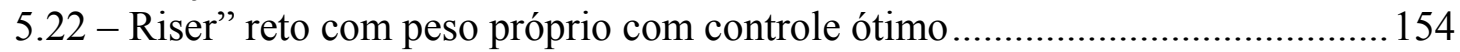

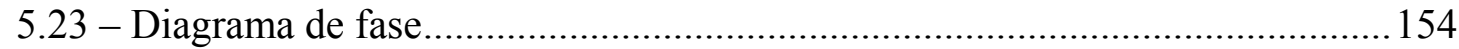

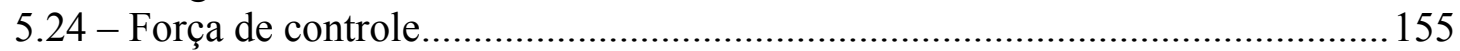

5.25 - Riser" reto com peso próprio com controle ótimo ....................................... 156

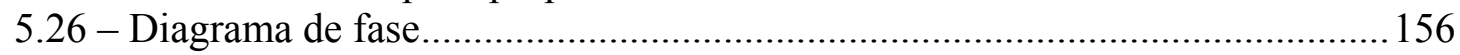

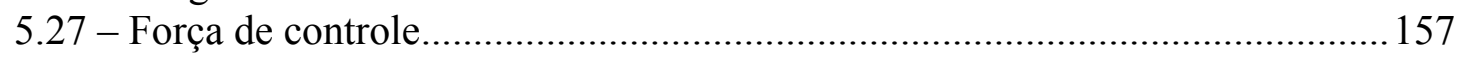

5.28 - Resposta no tempo, riser reto com controle.............................................. 159

5.29 - Diagrama de Fase, riser reto com controle ................................................. 159 


\section{Lista de Tabelas}

3.1 - Propriedades físicas do riser 68

3.2 - Propriedades físicas do riser. .84

3.3 - Propriedades físicas do riser. 102

4.1 - Propriedades físicas do riser 


\section{Lista de Símbolos}

\begin{tabular}{|c|c|}
\hline$a_{i j}, b_{i j}$ & coeficientes das relações modais \\
\hline$a^{i}$ & constante \\
\hline$b^{i}$ & constante \\
\hline $\mathrm{c}_{\mathrm{r}}$ & escalares \\
\hline $\mathrm{f}_{\mathrm{i}}$ & aceleração correspondente à i-ésima coordenada generalizada \\
\hline$f_{u}$ & força de controle modal \\
\hline $\mathrm{k}$ & Rigidez da mola \\
\hline l & comprimento do elemento \\
\hline $\mathrm{m}$ & massa concentrada \\
\hline $\mathrm{m}_{\mathrm{rs}}^{0}$ & elemento da matriz de massa da dinâmica linear \\
\hline $\mathrm{n}_{\mathrm{ij}}$ & elemento da matriz de relações modais \\
\hline$p_{i}$ & deslocamentos generalizados \\
\hline $\mathrm{s}$ & constante \\
\hline$S^{r}$ & Número complexo associado ao modo " $r$ " \\
\hline$\overline{\mathrm{S}}^{\mathrm{r}}$ & Número complexo conjugado associado ao modo " $\mathrm{r}$ " \\
\hline $\mathrm{u}, U$ & $\begin{array}{l}\text { deslocamento modal, deslocamento longitudinal de um ponto da barra, força } \\
\text { de controle, velocidade da corrente }\end{array}$ \\
\hline$\overline{\mathrm{u}}$ & deslocamento longitudinal de um ponto do eixo da barra \\
\hline $\mathrm{u}_{\mathrm{rs}}^{0}$ & elemento da matriz de rigidez da teoria linear \\
\hline $\mathrm{V}$ & velocidade modal, deslocamento transversal de um ponto da barra \\
\hline$\overline{\mathrm{v}}$ & deslocamento transversal de um ponto do eixo da barra \\
\hline $\mathrm{x}$ & abscissa axial, deslocamento em relação à posição indeformada da mola \\
\hline $\mathrm{x}_{\mathrm{I}}$ & Posição de equilíbrio estático estável \\
\hline $\mathrm{X}_{\mathrm{II}}$ & Posição de equilíbrio estático instável \\
\hline $\mathrm{x}_{\text {III }}$ & Posição de equilíbrio estático estável \\
\hline $\mathrm{x}_{0 \lim }$ & amplitude correspondente à freqüência nula \\
\hline $\mathrm{x}_{\lim }$ & amplitude correspondente à freqüência nula \\
\hline $\mathrm{x}_{0 \lim }^{\prime}$ & amplitude correspondente à freqüência nula \\
\hline
\end{tabular}




\begin{tabular}{|c|c|}
\hline $\mathrm{y}_{0}$ & Número real \\
\hline $\mathrm{t}$ & Tempo \\
\hline A & área da seção transversal \\
\hline$A_{i}$ & termo constante da equação de movimento de primeira ordem \\
\hline$B_{i j}$ & coeficientes de termos lineares da equação de primeira ordem \\
\hline $\mathrm{C}_{\mathrm{ij}}$ & coeficientes de termos lineares da equação de primeira ordem \\
\hline $\mathrm{D}_{\mathrm{ij}}^{0}$ & coeficientes de termos lineares das equações de segunda ordem \\
\hline $\mathrm{D}_{\mathrm{ijk}}^{1}$ & coeficientes de termos quadráticos das equações de segunda ordem \\
\hline $\mathrm{D}_{\mathrm{ijk} \mathrm{k}}^{2}$ & coeficientes de termos cúbicos das equações de segunda ordem \\
\hline $\mathrm{E}$ & Módulo de elasticidade do material \\
\hline $\mathrm{E}_{\mathrm{ijm}}$ & coeficientes de termos quadráticos da equação de primeira ordem \\
\hline $\mathrm{F}_{\mathrm{i}}$ & i-ésima componente do campo vetorial $\mathbf{F}$ \\
\hline $\mathrm{F}_{\mathrm{ijm}}$ & coeficientes de termos quadráticos da equação de primeira ordem \\
\hline $\mathrm{G}_{\mathrm{ijm}}$ & coeficientes de termos quadráticos da equação de primeira ordem \\
\hline $\mathrm{H}_{\mathrm{ijmp}}$ & coeficientes de termos de quarta ordem da equação de movimento \\
\hline I & momento de inércia da seção transversal \\
\hline $\mathrm{K}_{\mathrm{ij}}^{0}$ & coeficientes de termos lineares das equações de segunda ordem \\
\hline $\mathrm{K}_{\mathrm{ij \textrm {k }}}^{1}$ & coeficientes de termos quadráticos das equações de segunda ordem \\
\hline $\mathrm{K}_{\mathrm{ijkl}}^{2}$ & coeficientes de termos cúbicos das equações de segunda ordem \\
\hline $\mathrm{L}$ & comprimento da barra \\
\hline $\mathrm{L}_{\mathrm{ijmp}}$ & coeficientes de termos de quarta ordem da equação de movimento \\
\hline $\mathrm{M}_{\mathrm{ij}}^{0}$ & coeficientes de termos lineares das equações de segunda ordem \\
\hline $\mathrm{M}_{\mathrm{ijk}}^{1}$ & coeficientes de termos quadráticos das equações de segunda ordem \\
\hline $\mathrm{M}_{\mathrm{ijk} k \mathrm{l}}^{2}$ & coeficientes de termos cúbicos das equações de segunda ordem \\
\hline $\mathrm{N}_{\mathrm{ijmp}}$ & coeficientes de termos de quarta ordem da equação de movimento \\
\hline $\mathrm{R}_{\mathrm{ijmp}}$ & coeficientes de termos de quarta ordem da equação de movimento \\
\hline $\mathrm{S}_{\mathrm{r}}$ & subespaço do espaço de fase associado ao r-ésimo modo \\
\hline
\end{tabular}




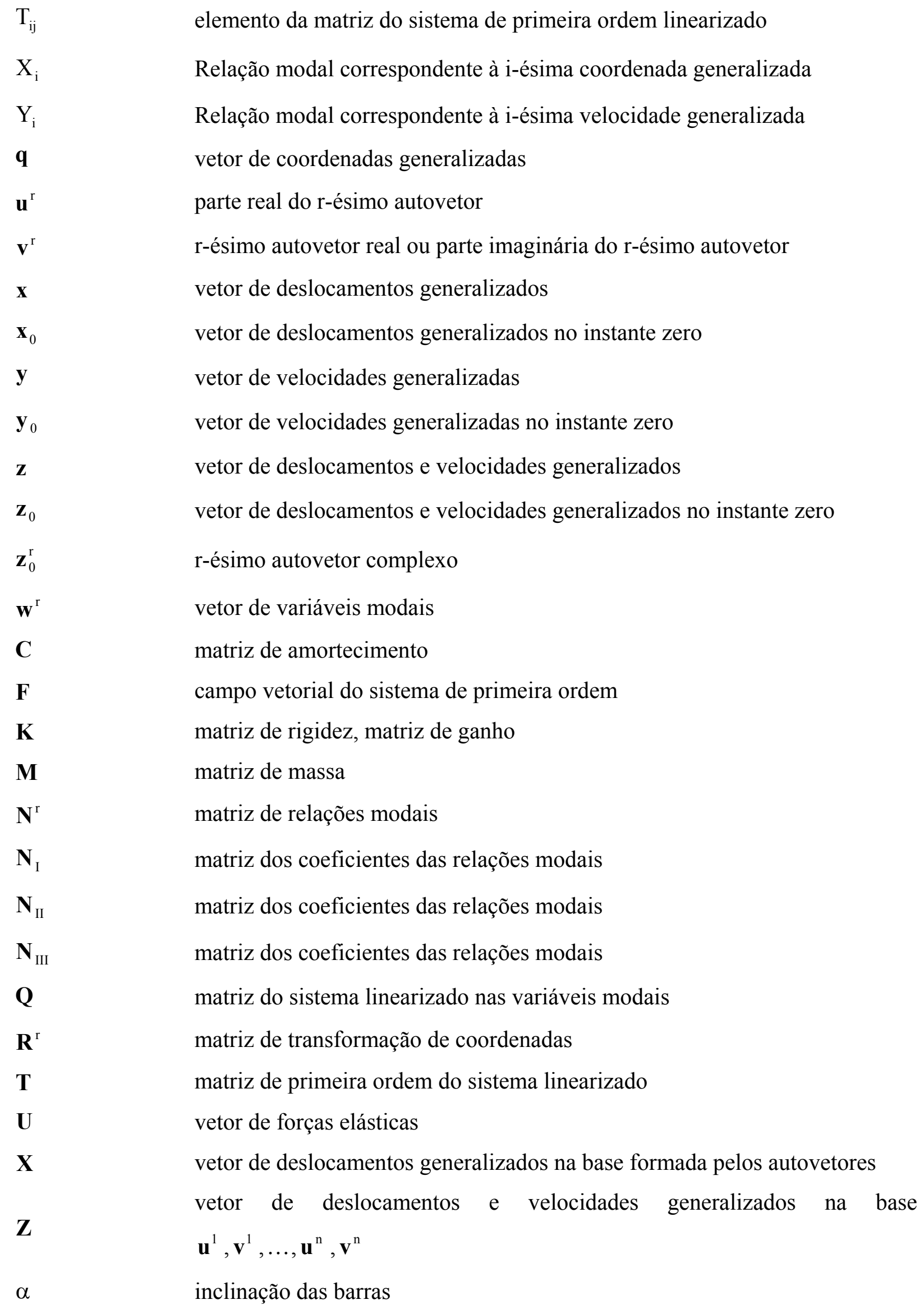




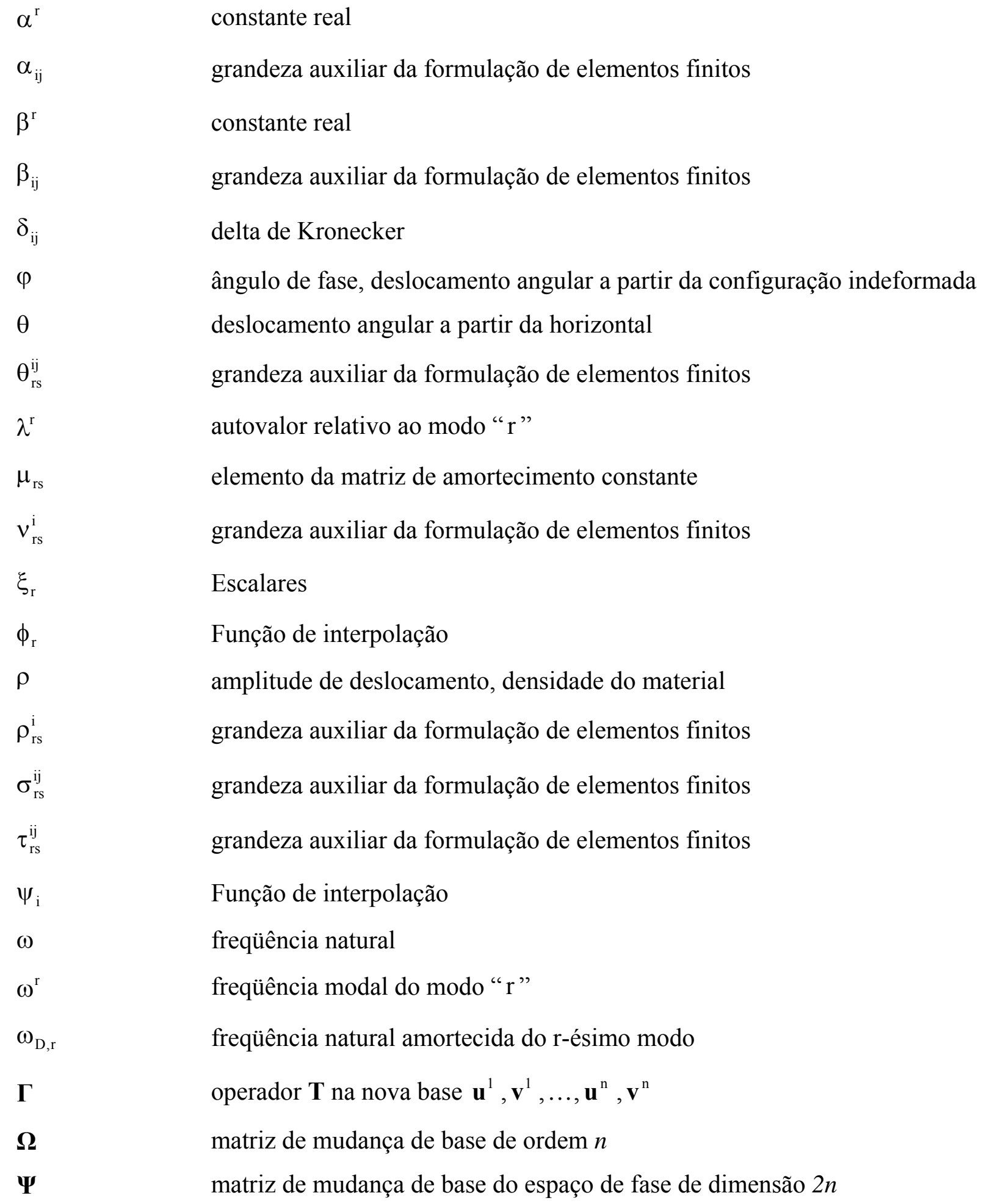




\section{Resumo}

Este trabalho tem por objetivos: o estudo do comportamento dinâmico nãolinear de risers oceânicos, utilizando modos não-lineares de vibração, e o controle ativo das vibrações neles induzidas. Uma formulação não-linear do método dos elementos finitos é utilizada para a modelagem dos sistemas estruturais com vários graus de liberdade. Pela aplicação do método das variedades invariantes ao modelo de elementos finitos, determinam-se os modos normais não-lineares. Para o caso de ressonâncias internas, pode ser necessário determinar multi-modos não-lineares, o que se faz pela aplicação do método das múltiplas escalas.

Apreciável esforço científico ainda é necessário para uma compreensão profunda da dinâmica de risers. Muitas das questões em aberto estão relacionadas a aplicações em águas ultraprofundas. Recentemente, novas reservas de óleo e gás foram descobertas no mar brasileiro em águas ultraprofundas, com lâminas d'água superiores a $2500 \mathrm{~m}$. Assim, a indústria offshore se depara com um novo conjunto de problemas e desafios relacionados à dinâmica não-linear de risers. Desta forma, a análise modal não-linear pode ter um importante papel na modelagem de RiserTower's, SCR's (Steel Catenary Riser), entre outros sistemas oceânicos. Um procedimento completo consideraria diversos aspectos como movimentos induzidos por ondas (WIM - Wave Induced Motion) e por correntes oceânicas. Nesta última categoria, ressaltam-se as vibrações induzidas por vórtices (VIV - Vortex Induced Vibration). A todos esses fenômenos se somam o escoamento interno, deslocamento imposto, contato com o solo marinho, etc.

Nota-se que a análise modal não-linear é adequada para representar o comportamento da dinâmica global dos risers por meio de modelos matemáticos com poucos graus de liberdade. Diferenças relevantes entre as abordagens linear e nãolinear são encontradas na forma modal e nos diagramas de fase. Tais diferenças podem ser importantes quando o arrasto e a inércia do fluido forem considerados. Multi-modos são utilizados para abordar ressonâncias internas utilizando análises acopladas que são importantes no caso de excitação multi-modal de modos altos por VIV. De fato, modos não-lineares podem ser úteis na elaboração de modelos matematicamente reduzidos que apresentem uma resposta qualitativa e quantitativamente coerente com a resposta da análise de risers sob carregamentos reais.

A análise modal não-linear é uma técnica inovadora que permite um aprofundamento do entendimento do comportamento da dinâmica dos risers e a elaboração de modelos matemáticos com poucos graus de liberdade. Por serem modelos menores, consomem menos tempo de processamento levando a respostas mais rápidas, essenciais para sistemas de controle eficientes. Alguns exemplos de sistemas de controle são estudados neste trabalho. 


\section{Abstract}

The present work has the following objectives: deep understanding of the nonlinear structural behaviour of riser dynamics, by using non-linear modal analysis, and the study of these structural systems when an active control system is coupled. The finite element method provides a set of non-linear equations of motion for the structural system considered. These non-linear equations of motion are handled by the so-called invariant manifold approach: non-linear vibration modes are defined as movements whose trajectories in phase space are confined to a two-dimensional invariant manifold. In addition, the method of multiple scales is used to determine the non-linear multi-modes, when internal resonances come into play.

Riser dynamics still demands great research efforts, many of the open topics being related to deep and ultra-deep water applications. The offshore industry has already begun to explore in water depths at the limits of the current technology and has plans to access sites over 2500 metre-deep. Therefore, offshore industry will face new challenges concerning the non-linear dynamics of risers. Non-linear modal analysis will play an important role in the project development of hybrid towers, SCR's and so on. A complete design procedure considers several issues associated with the dynamic response to wave-induced motions (WIM) of the Floating Production Unity (FPU) and to ocean currents. In this latter category one may cite vortex-induced motions (VIM) of spar-type FPUs and vortex-induced vibration (VIV).

Results point out that non-linear modal analysis is adequate to capture the global dynamic behaviour of risers. Relevant differences with respect to linear analysis might then appear in the modal shape and phase trajectories. Such differences might be important when drag and inertia fluid loads will be further considered. Multi-modes should be considered to approach internal resonance. Coupling analysing may be relevant in the case of multi-modal excitation of higher modes in VIV. Non-linear modes are believed to play an important role in degree-of-freedom model reduction, thus allowing for good qualitative and quantitative response analysis of risers under sea loading.

Non-linear modal analysis is an innovative technique that allows for deeper understanding of riser dynamics. In addition, reduced models can be found in order to diminish the processing time. Reducing the calculation time is very important when control systems are considered. This work studies some examples of risers that are controlled by active systems, which are considerably affected by the processing time. 


\section{Capítulo 1}

\section{Introdução}

A exploração de óleo e gás em águas profundas e ultraprofundas tem sido alvo de atenção e de investimentos crescentes. Alguns fatores que impulsionam tal fenômeno estão associados ao declínio das taxas de crescimento de produção de óleo e ao aumento significativo do preço do barril de petróleo, quando se observa a tendência dominante das últimas décadas. Este último pode ser associado à falta de investimentos em prospecção, produção, refinarias, baixa capacidade de armazenamento, aceleração do consumo de óleo e derivados e esgotamento das reservas de energia não-renováveis.

O declínio na produção está associado à maturação de importantes campos em produção, especialmente, nos países ditos de primeiro mundo que, via de regra, são os maiores consumidores. Como resposta a esta situação, diversas companhias saem em busca de novas bacias produtoras, muitas vezes localizadas em países em desenvolvimento. Entretanto, tais empresas encontram-se em desvantagem estratégica frente às companhias nacionais que, obviamente, possuem o apoio de seus governos e, além disso, possuem investimentos extensivos nas suas regiões.

Uma maneira de contornar tais dificuldades se dá pela formação de consórcios entre empresas estrangeiras e as companhias nacionais, onde estas últimas possuem participação majoritária. Estes blocos mostram-se interessantes uma vez que viabilizam os desenvolvimentos de novas tecnologias, além do intercâmbio das tecnologias dentro e fora do país.

O Mar do Norte, por exemplo, atingiu seu pico de produção em 1999. Da mesma forma, outros importantes campos produtores atingiram a maturidade e impulsionaram a indústria do óleo para novas descobertas, dentre as quais se destacam países como Angola, Nigéria e Brasil. Neste último ressalta-se a recente descoberta na Bacia de Santos, o campo de Tupi. Tal reserva localiza-se a aproximadamente $6000 \mathrm{~m}$ 
da superfície do mar, sendo que desta profundidade total $2000 \mathrm{~m}$ correspondem à lâmina d'água.

Como já mencionado anteriormente, devido às condições econômicas e geopolíticas, diversas instituições privadas e públicas têm lançado esforços para o desenvolvimento de tecnologias para solução dos problemas originados da exploração em condições cada vez mais severas. Este cenário de desafios justifica o presente trabalho na medida em que este desenvolve uma ferramenta para a análise de determinados sistemas estruturais submetidos a condições extremas de solicitação oriundas da exploração em água profundas.

A exploração em águas ultraprofundas leva os sistemas estruturais a regimes não-lineares de reposta dinâmica que antes podiam ser negligenciados em condições mais amenas de solicitação. No entanto, tais efeitos não-lineares tornam-se significativos em função, por exemplo, do aumento do comprimento dos risers, das velocidades das correntes oceânicas, do escoamento interno, da diversidade e intensidade das ondas, das condições de contacto com o leito marinho, etc. Como resultado de tais efeitos, fenômenos indesejados podem ocorrer, tais como a redução da vida útil dos elementos, falhas, infiltrações e vazamentos.

Diante deste cenário, fazem-se necessários modelos matemáticos $\mathrm{e}$ computacionais cada vez mais sofisticados capazes de representar adequadamente os sistemas reais. A partir de tais modelos, novos procedimentos e códigos podem ser elaborados levando em conta efeitos, até então, não completamente conhecidos.

O trabalho utiliza o método dos elementos finitos para a discretização inicial dos sistemas estruturais, considerando a complexidade de vinculação, geometria e carregamento típica dos projetos de engenharia. Em seguida, a análise modal nãolinear é aplicada para determinação das propriedades dinâmicas desses sistemas.

Projetos típicos de engenharia offshore apresentam complexidade elevada. Isto se deve à variedade de seus carregamentos, que podem ser oriundos de diversas origens, tais como ondas, correntes, ventos, etc., e à diversidade de sua geometria e 
propriedades físicas muitas vezes não-lineares. Geometrias complexas levam a modelos matemáticos com um grande número de graus de liberdade.

Este elevado número de graus de liberdade demanda considerável esforço computacional. Este esforço é tanto maior na medida em que condições de contorno complexas como as TDZ’s (Touch Down Zones), não-linearidades físicas e nãolinearidades geométricas são levadas em consideração. Além de aumentar o tempo de cálculo computacional, essa massa de dados dificulta a compreensão do comportamento principal dos sistemas estruturais ou a compreensão dos efeitos mais significativos na dinâmica de risers.

A abordagem via modos não-lineares de vibração tem por objetivo a redução do número de graus de liberdade dos modelos estruturais sem perder a capacidade de descrição do seu comportamento, ou característica, principal dos sistemas. Por esta abordagem alguns poucos modos não-lineares de vibração seriam suficientes para capturar o comportamento dos sistemas. Modos não-lineares são capazes de capturar a influência de modos superiores devido à consideração de termos não-lineares na sua formulação. Assim, um único modo não-linear pode guardar informações de dois ou mais modos lineares.

O elevado número de graus de liberdade de um sistema pode dificultar substancialmente a análise, por exemplo, de um sistema de controle ativo. Para este último a velocidade de obtenção da resposta é crítica para eficiência do controle estrutural. Quando maior o número de graus de liberdade, maior o tempo de cálculo e, portanto, maior o atraso na obtenção da resposta. Modos não-lineares podem desempenhar papel crucial na redução do volume de cálculos, levando a respostas mais rápidas do sistema de controle.

Além disso, os modos não-lineares de vibração permitem uma compreensão qualitativa da enorme massa de dados fornecida no modelo de elementos finitos. $\mathrm{O}$ volume de dados resultantes de uma análise no domínio do tempo é considerável mesmo para uma análise linear, sendo ainda maior para as análises não-lineares. A tarefa de interpretação dessa massa de dados é por vezes árdua e demorada. Assim os modos não-lineares poderiam ser utilizados como uma interessante ferramenta na 
compreensão da dinâmica não-linear dessas repostas no domínio do tempo. Não obstante, os modelos reduzidos oriundos dos modos não-lineares de vibração devem ser comparados com os resultados do modelo completo de elementos finitos para sua validação e calibragem.

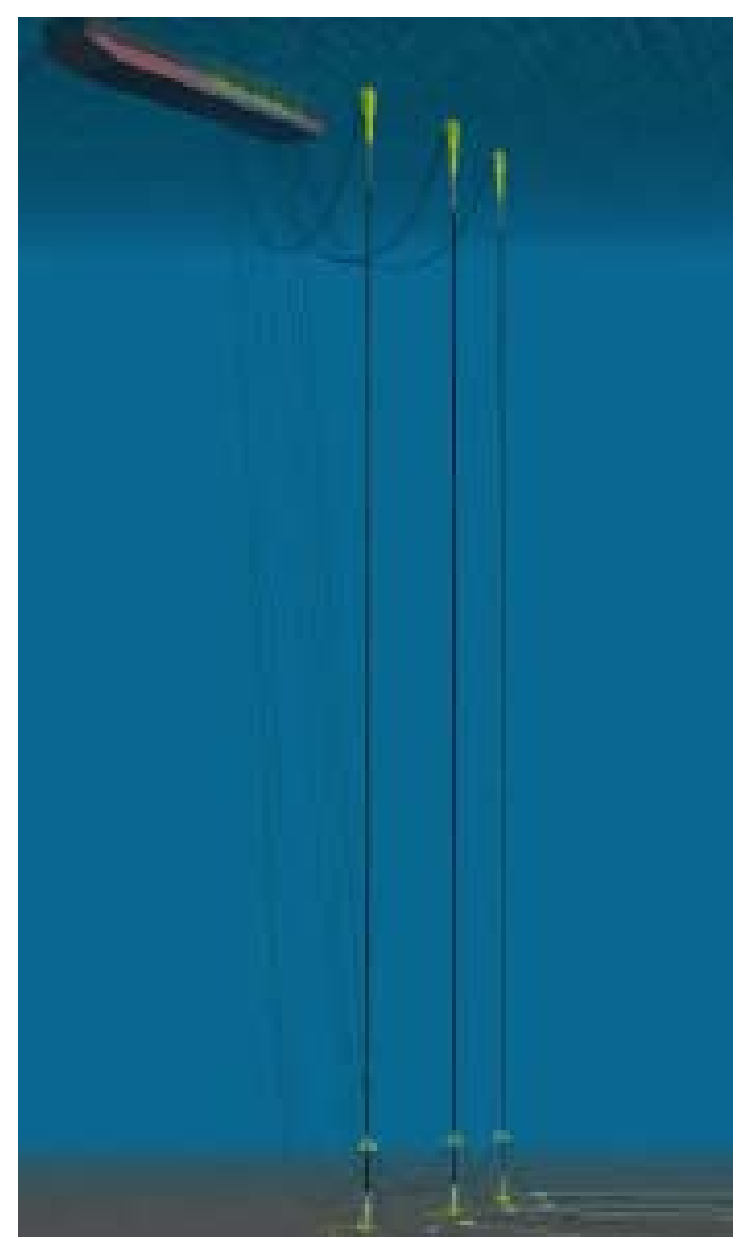

Figura 1.1 - Campo de exploração em águas profundas com Riser Towers.

A seleção do conceito de riser mais eficaz para um determinado campo de exploração é de extrema importância em projetos para águas profundas. Dois importantes candidatos são: o riser de aço em catenária (Steel Catenary Riser - SCR), que é lançado individualmente, e a torre de risers (Riser Tower), que consiste em um conjunto de risers empacotados numa torre vertical.

Ao longo dos últimos quinze anos aproximadamente, muitos esforços têm sido direcionados para o desenvolvimento de sistemas de exploração offshore em águas profundas e ultraprofundas. Inicialmente os risers utilizados para águas rasas foram utilizados para a exploração de reservas em águas profundas. Entretanto, a 
necessidade de desenvolver campos com profundidades superiores a mil metros de profundidade levou ao desenvolvimento de novos conceitos de risers, por exemplo, os SCR's e os Riser Towers.

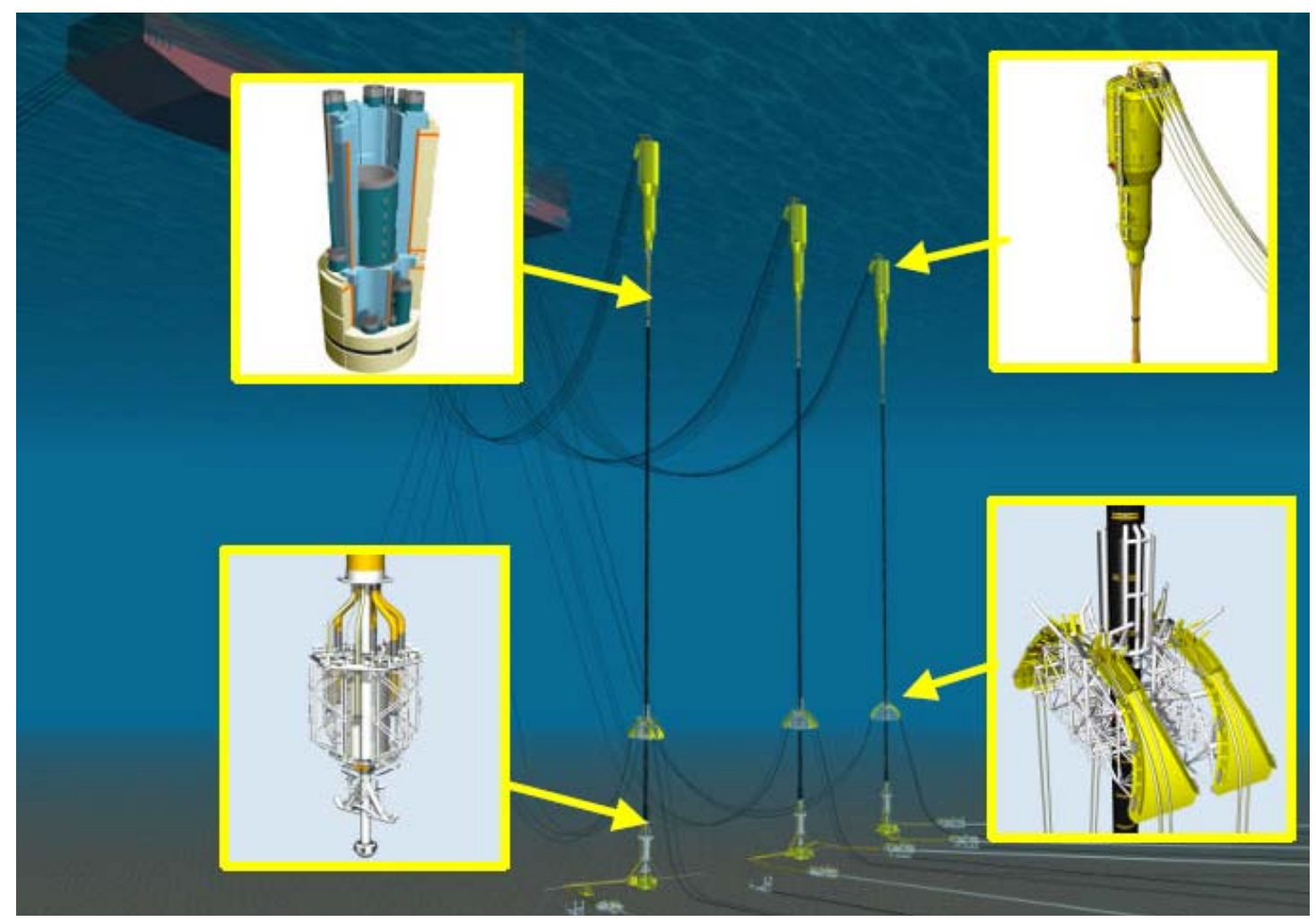

Figura 1.2 - Detalhes de um Riser Tower.

A figura 1.1 (ver [76]) apresenta os Riser Towers de forma geral, enquanto a figura 1.2 (ver [76]) mostra detalhes desse sistema. Já a figura 1.3 apresenta um campo de exploração com SCR's. A representação esquemática de um SCR pode ser apreciada na figura 1.4 .

O conceito de Riser Tower consiste em um conjunto de risers empacotados juntos em torno de um tubo central, que é fabricado e montado onshore. Este conjunto é utilizado para transportar fluidos ou para garantir adequada rigidez mecânica, ou para ambos. Esta coluna vertical é tracionada por meio de uma estrutura flutuante localizada no topo da coluna ou distribuída ao longo da coluna. A interface com o fundo do mar é estática e consiste em uma âncora sobre a qual a coluna de risers se conecta por meio de um dispositivo mecânico desenvolvido para absorver a inclinação devida ao movimento dinâmico da torre (flex joint). No fundo do mar os risers verticais e os flowlines horizontais se interconectam através de spools e diversas 
outras conexões. O topo do Riser Tower, que é posicionado a uma profundidade ótima para minimizar os efeitos das ondas e das correntes, é conectado à unidade flutuante através de uma série de tubos curtos flexíveis. Esses tubos são flexíveis e desenvolvidos para garantir a continuidade do fluxo e para absorver os movimentos de heave da unidade flutuante. O conceito de Riser Tower tem sua origem no Golfo do México, onde em 1988 um conjunto de risers empacotados foi instalado no Bloco 29 (The Green Canyon - Block 29).

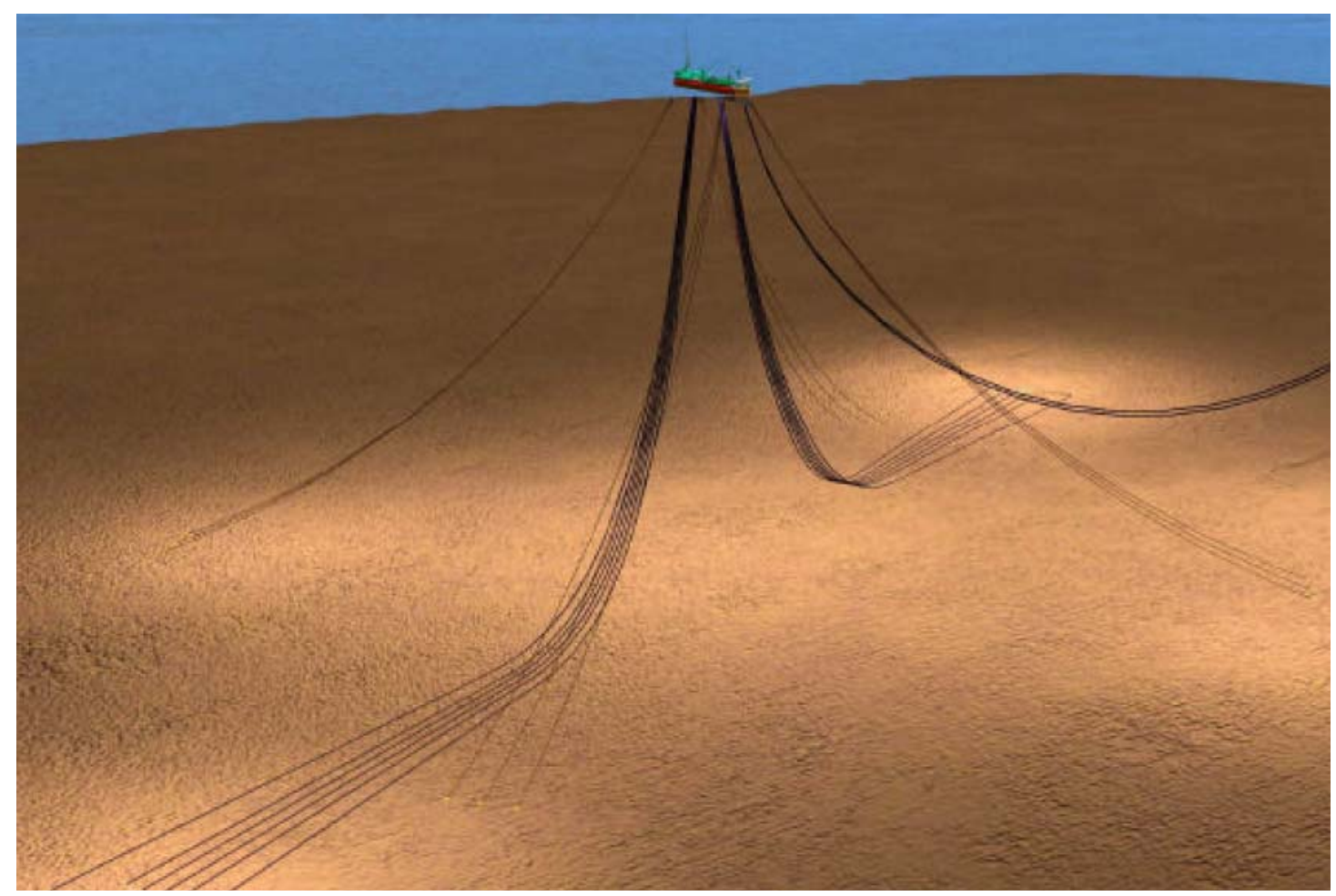

Figura 1.3 - Campo de exploração em águas profundas com SCR’s (ver [76]).

O conceito de SCR é, basicamente, uma extensão do conceito de steel flowline, que é levado à superfície do mar e conectado à unidade flutuante. Conseqüentemente, cada flowline exige um SCR individualmente. O SCR é instalado utilizando a tecnologia de pipelay que requer soldagem offshore, que é um ponto crítico. $\mathrm{O}$ primeiro SCR foi instalado no Golfo do México, no desenvolvimento de Auger, em 1994. Desde então, um número considerável tem sido instalado no Golfo do México e, principalmente, no Brasil. 


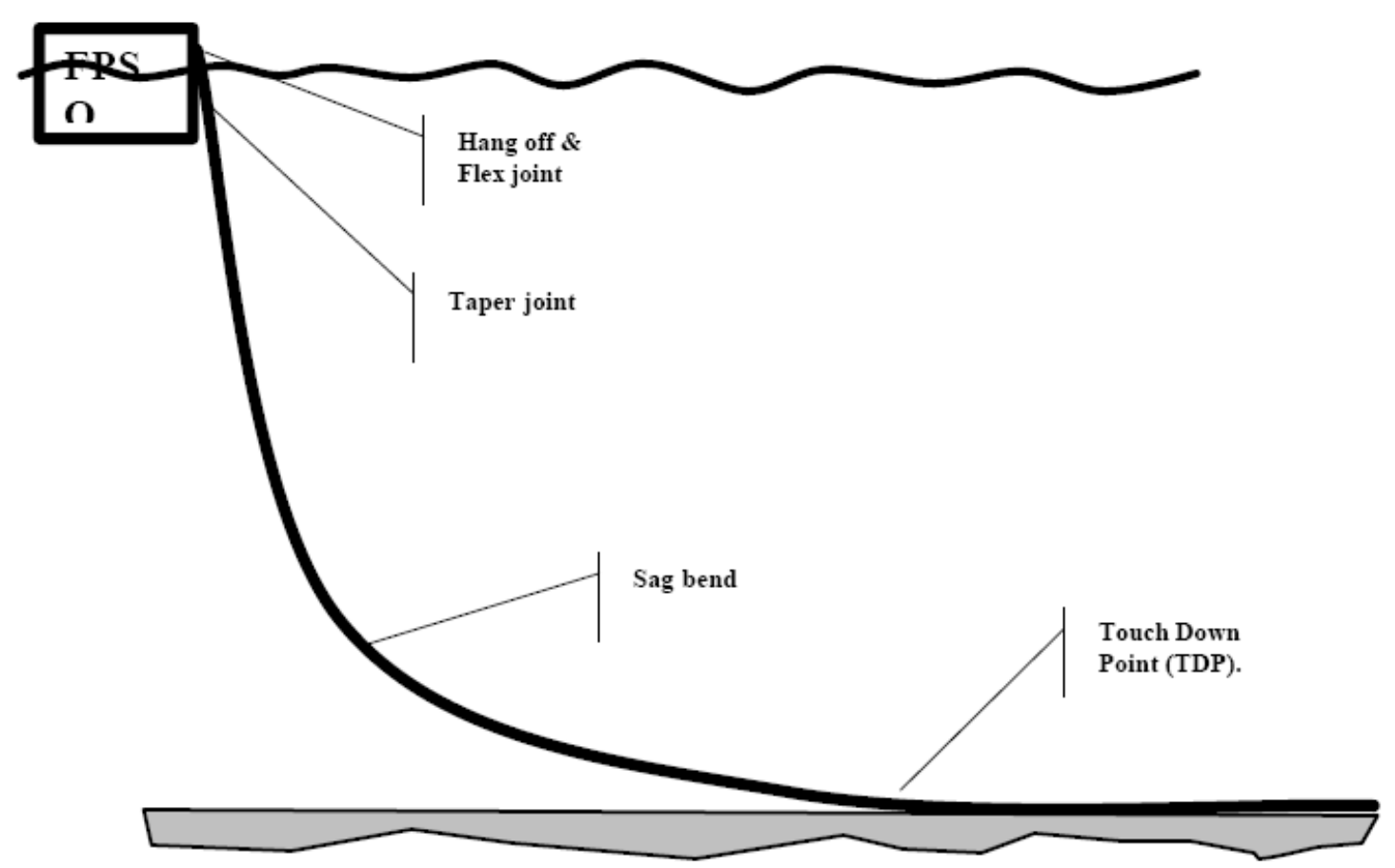

Figura 1.4 - Figura esquemática de um SCR’s (ver [76]).

O SCR é um único tubo suspenso em catenária a partir do suporte da unidade flutuante. O SCR deixa a unidade flutuante em catenária até tocar o fundo mar quando segue horizontalmente, conforme figura 1.4. A interface com a unidade flutuante consiste em uma estrutura de hang off e uma junta flexível para absorver as variações dinâmicas de momento geradas pela unidade flutuante. A interface com o fundo do mar é dinâmica. O ponto de toque (Touch Down Point - TDP) pode se mover tanto axialmente quanto lateralmente. A essa região de toque dá-se o nome de Touch Down Zone - TDZ. Um sistema de ancoragem pode, também, ser incorporado para estabilizar o riser axialmente. Essa interação cria uma região bastante sensível à fadiga que, freqüentemente, requer análise, seleção de material e processo de fabricação especiais. SCR's são estruturas dominadas pela fadiga, com os carregamentos hidrodinâmicos provenientes de ondas e correntes, incluindo aqueles gerados pela vibração induzida por vórtices (Vortex Induced Vibration - VIV), desempenhando um papel significativo no seu projeto e posterior fabricação. Geralmente, SCR's são fabricados offshore utilizando uma embarcação especial que normalmente possui uma extensão de instalação. Em geral, SCR's precisam ser instalados uma vez que a unidade flutuante de produção esteja posicionada. Isto envolve uma operação complicada entre a embarcação de pipelay e a unidade 
flutuante de produção, sendo que a embarcação de pipelay trabalha bastante próxima da unidade de produção.

Até a atualidade, o conceito de Riser Tower tem sido uma solução que responde favoravelmente a certas exigências que o tornam uma solução competitiva contra os SCR's e flexíveis, por exemplo, quanto ao congestionamento do leito marinho e exigências de isolamento térmico. Entretanto, Riser Towers tendem a ser soluções mais caras até profundidades de pelo menos $1000 \mathrm{~m}$, com a solução em SCR geralmente estimada em $20 \%$ mais barata.

A figura 1.5 ilustra a evolução do custo versus a profundidade para ambos SCR e Riser Tower. O custo varia linearmente para ambos os sistemas com uma inclinação maior para o SCR. Quando alcançada uma profundidade crítica, a taxa de crescimento do custo cresce significativamente para o SCR devido aos equipamentos de instalação utilizados para suportar altas trações. A mudança da taxa de crescimento depende de outros fatores além da profundidade; no entanto, para um SCR típico, a mudança ocorre ao redor de $1800 \mathrm{~m}$ de profundidade. Uma mudança na taxa de crescimento de custo do Riser Tower também ocorre devido a mudanças nas exigências de projeto em função da profundidade. Tal aumento da taxa de crescimento de custo é esperada para uma profundidade ao redor de $2500 \mathrm{~m}$.

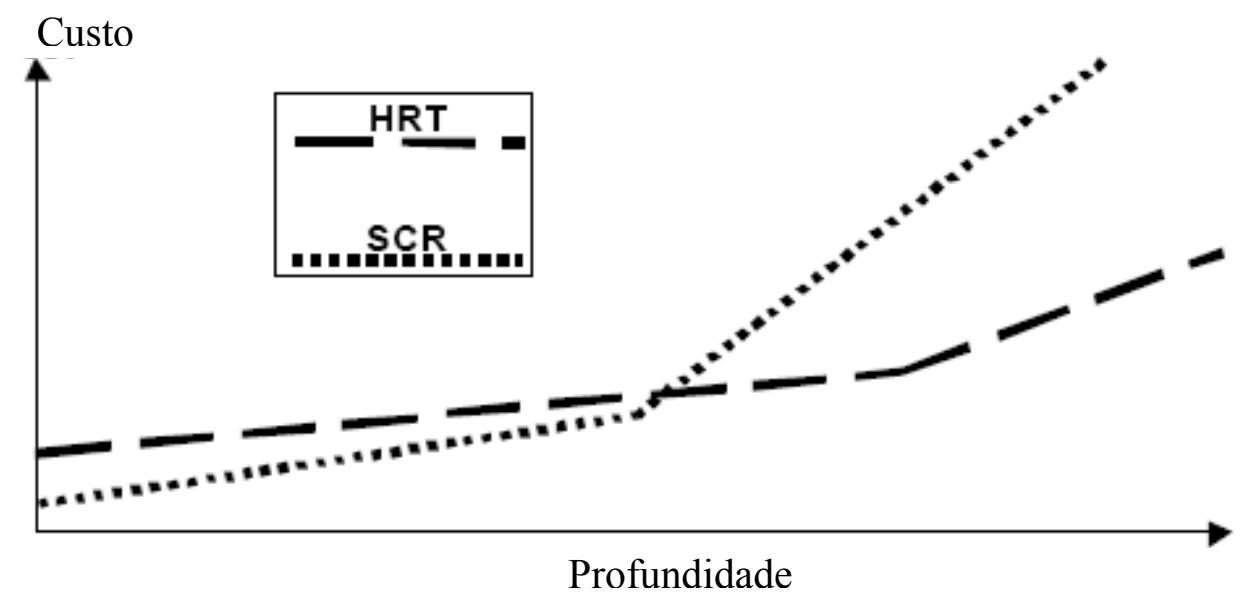

Figura 1.5 - Comparação de custo entre Riser Tower e SCR (ver [76]). 
Para os risers, de forma geral, o carregamento predominante é de natureza hidrodinâmica (Pesce [69]). Admitem-se para o projeto de risers as ondas de superfície, a correnteza oceânica e o vento. As ondas afetam o sistema estrutural de duas maneiras: uma diretamente, ao longo do comprimento do riser (mais propriamente junto à superfície, diminuindo sua influência com a profundidade), e outra indiretamente, pela alteração da posição da unidade flutuante, que serve de apoio à extremidade superior do riser, e pelo movimento imposto à unidade flutuante. A correnteza além de agir sobre a unidade flutuante, aciona o riser ao longo de seu comprimento, determinando a configuração estática e afetando a resposta dinâmica através das vibrações induzidas por vórtices (VIV). A ação do vento se dá de forma indireta através da movimentação da unidade flutuante. Há ainda o carregamento associado ao fluxo interno de óleo ou gás que pode ser relevante em determinadas condições.

Diversas escalas de tempo e comprimento estão presentes na dinâmica deste sistema complexo. A escala de tempo de variação da correnteza, por exemplo, é de cerca de dias ou de horas, em regiões influenciadas pela maré litorânea. As escalas de tempo características do vento são bem variadas, podendo ser bem pequenas (alguns segundos), quando associadas às rajadas. A escala de tempo característica dos movimentos das unidades flutuantes no plano horizontal (surge, sway, yaw), por sua vez, é de cerca de 2 a 3 minutos, posto que se caracterizam por apresentar elevadíssima inércia e pequena restauração propiciada pelo sistema de fundeio. Já os movimentos nos planos verticais (heave, roll, pitch) ocorrem em períodos característicos de alguns segundos (cerca de 20 para plataformas semi-submersíveis, 10 para navios e 2 segundos para TLP's).

Surge é o movimento de translação longitudinal; sway o lateral; yaw o de rotação em torno da vertical; heave corresponde à oscilação vertical; roll à rotação em torno do eixo longitudinal e pitch a rotação em torno do eixo transversal. 
A figura 1.6 apresenta a distribuição de energia de onda para o mar brasileiro na região da bacia de Campos. Como pode ser observado, trata-se de uma distribuição bi-modal com dois picos em torno das freqüências $0.1 \mathrm{~Hz}$ e $0.2 \mathrm{~Hz}$, correspondendo a períodos de $10 \mathrm{~s}$ e $5 \mathrm{~s}$, respectivamente. Além de bi-modal, as ondas na região da bacia de Campos também são bi-direcionais.

Em geral, as ondas de superfície caracterizam-se por espectros de energia contínuos de largura de banda relativamente estreita. Ondas de grande amplitude e comprimento apresentam períodos típicos da ordem de 10 a 15 segundos e ondas de pequenas amplitudes e pequenos comprimentos, períodos de cerca de 3 a 7 segundos. No entanto, diversos casos de carregamento devem ser considerados na estimativa da vida à fadiga do riser e, em alguns casos, essas relações entre períodos e amplitudes podem ser invertidas.

Energia de Onda

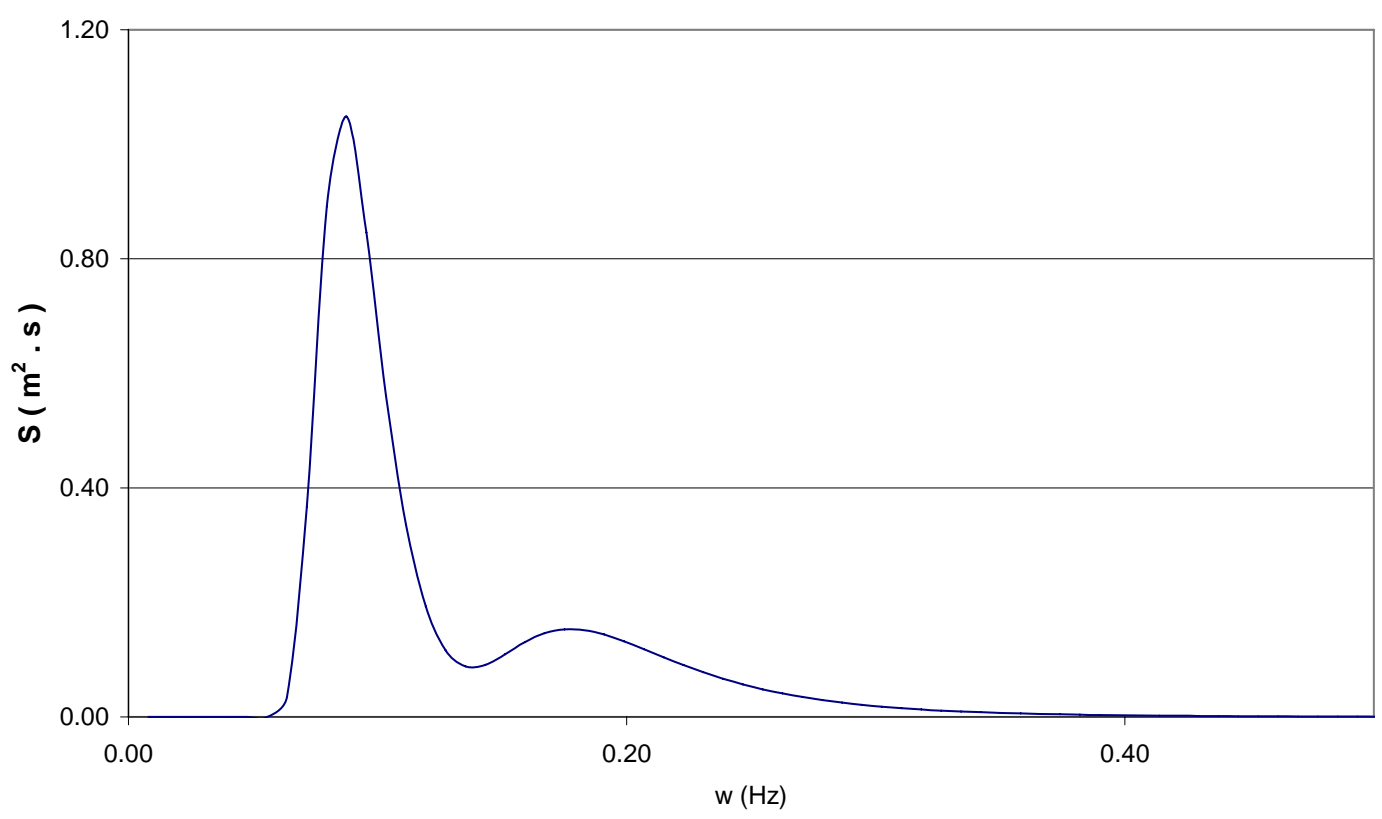

Figura 1.6 - Espectro de energia de onda característica para mar bi-modal.

Com relação às linhas elásticas dos risers, três são as principais escalas de comprimento presentes. A primeira é o próprio comprimento suspenso. A segunda, o diâmetro da linha, muito pequeno se comparado ao comprimento. A terceira escala é denominada "comprimento de flexão", e mede a distância característica dentro da qual o comportamento de viga, dominante junto às extremidades ou restrições geométricas 
intermediárias, é compatibilizado ao comportamento de catenária, que rege o comportamento global da linha. Os períodos naturais dos modos transversais baixos, com semi-comprimento de onda da ordem da profundidade, são dominados pela rigidez geométrica. Dependem do inverso da raiz quadrada da profundidade. Os modos transversais altos, com semi-comprimento de onda da ordem do comprimento de flexão, são dominados pela rigidez flexional, no entanto. Os modos longitudinais, associados à dinâmica de barra, são dominados pela rigidez axial. O período associado ao fenômeno de liberação de vórtices depende, finalmente, do diâmetro da linha e da intensidade da correnteza. Valores típicos podem ser encontrados na faixa abaixo de 20 segundos (Pesce [69]). 


\subsection{Objetivo}

Esta pesquisa tem por objetivo o estudo da dinâmica e do controle ativo de risers com comportamento não-linear, utilizando modos não-lineares de vibração para obtenção de modelos matemáticos reduzidos (com poucos graus de liberdade). Todos os modelos estudados são planos. Os modos normais não-lineares de vibração são obtidos utilizando-se a abordagem por variedades invariantes. Os multi-modos nãolineares de vibração são encontrados utilizando-se o método das múltiplas escalas. São estudadas ressonâncias internas por meio de modelo de modos acoplados.

Estudam-se primeiramente risers retos por uma abordagem analítica. As equações de movimento, os modos normais e os multi-modos são determinados analiticamente. Depois, para o mesmo sistema estrutural, determinam-se as equações de movimento, os modos normais e os multi-modos utilizando-se um sistema discretizado pelo método dos elementos finitos. As respostas das duas abordagens, analítica e método dos elementos finitos (MEF), são comparadas para diferentes geometrias e condições iniciais para vibrações livres.

Posteriormente abordam-se risers em catenária, porém apenas através do MEF. Determinam-se as formas modais, as respostas temporais e diagramas de fase para diferentes amplitudes. Em função destas últimas, encontram-se diferentes freqüências que são analisadas e relacionadas com diferentes propriedades e condições de contorno dos risers em estudo.

A partir das equações modais não-lineares dos risers introduz-se um sistema de controle ativo. O método de controle é o de alocação de pólos. O observador utilizado é o de Luenberger. Serão utilizados ganho linear e observador também linear, apesar de o sistema estrutural ser não-linear. Destaca-se a utilização de controle ótimo para alocação dos pólos. Utiliza-se o Regulador Linear Quadrático Determinístico (Linear Quadratic Regulator - LQR). As expressões finais, demonstrações e implementação computacional são apresentadas. Primeiramente, aborda-se a teoria considerando sistemas lineares e posteriormente estendem-se os conceitos para os sistemas não-lineares. 


\subsection{Justificativa}

Como abordado na introdução, devido às condições econômicas e geopolíticas correntes, o desenvolvimento de tecnologias para produção em águas profundas é prioridade na indústria petrolífera. Os aspectos técnicos envolvidos na execução de empreendimentos exploratórios cada vez mais ousados se mostram desafiadores. Este cenário de desafios justifica a presente pesquisa uma vez que esta tem por objetivo o desenvolvimento de ferramentas computacionais aplicadas à análise dinâmica nãolinear de risers e, além disso, à compreensão dos fenômenos envolvidos.

Primeiramente, apresenta-se um software de cálculo automático capaz de calcular os modos não-lineares normais de estruturas reticuladas sob protensão inicial. Este software, obviamente, é adequado para a determinação dos modos não-lineares de risers. Apenas modelos planos são processados pelo software.

Desenvolve-se, também, a análise e compreensão da dinâmica. Tais estudos se processam pela determinação de resposta no tempo e diagramas de fase que mostram as variações da freqüência com amplitude, valores de amplitudes máximos e mínimos, participação dos sistemas de amarração, etc.

Para o estudo do controle ativo de risers, utilizam-se rotinas em programas de matemática simbólica para o desenvolvimento dos observadores de estado. Os sistemas com controle são simulados e suas respostas são analisadas, de forma a mostrar a influência dos controles na dinâmica global. 


\subsection{Revisão Bibliográfica}

Rosenberg [75], definiu modos de vibração como sendo vibrações em uníssono de um sistema autônomo admissível. Os sistemas admissíveis são tais que, na ausência de esforços externos dinâmicos, possuem uma única configuração de equilíbrio, sendo permitido executar vibrações livres em torno da mesma e, na presença de excitação externa, realizar vibrações forçadas (em regime permanente). Por sua vez, as vibrações em uníssono devem ter as seguintes propriedades: o movimento das massas é equiperiódico; durante metade do período, o sistema passa precisamente uma vez pela configuração de equilíbrio; em um quarto de período, as velocidades das massas passam simultaneamente por zero, e os deslocamentos atingem os seus máximos; e o deslocamento de qualquer massa, em qualquer instante, pode ser determinado unicamente pelo deslocamento de uma das massas. Rosenberg aplicou o método geométrico, que consiste em primeiro calcular as relações entre as coordenadas generalizadas e, depois, a frequência modal. Uma extensão desta discussão pode ser encontrada em Pak e Rosenberg [66] (1968).

Shaw e Pierre (1991) [79], abordaram modos de vibração através das variedades invariantes. Na análise linear, modos de vibração são determinados a partir da solução de um problema de autovalores, sendo que os respectivos autovetores determinam as formas modais. Porém, Shaw e Pierre observaram que tal solução está contida numa superfície plana bidimensional no espaço de fase. Desta forma, sugeriram que a trajetória da solução modal de um sistema não-linear deveria estar contida numa superfície bidimensional no espaço de fase, que não necessariamente seria um plano. Isto significa dizer que, numa extensão dos modos lineares, as coordenadas generalizadas guardam entre si relações não-lineares para os sistemas não-lineares.

Em outros trabalhos, Shaw e Pierre, [80] (1993), [81] (1994) e [82] (1999), abordam sistemas massa-mola não-lineares através das variedades invariantes determinando os modos normais não-lineares desses sistemas estruturais. Também, abordam modelos reduzidos utilizando modos não-lineares. 
Nayfeh [61], em 1994, aborda problemas com vigas através de modos nãolineares e método das múltiplas escalas. Para determinar a dinâmica da variedade invariante é necessário resolver a equação do oscilador modal equivalente, o que Nayfeh faz através do método das múltiplas escalas.

Em outras publicações, Nayfeh [62] (1995), [63] (2000), utilizando o método das múltiplas escalas [60], determina os modos não-lineares de uma barra simplesmente engastada e estuda interações não-lineares analiticamente, computacionalmente e experimentalmente.

Outros autores se dedicam ao estudo de expansões locais dos modos nãolineares e à analise da estabilidade e outros métodos de análise dinâmica não-linear em [32], [45], [46], [54], [57], [58], [59] e [85]. Trabalhos com sistemas massa-mola e modos não-lineares podem ser encontrados em Vakakis [92] e [93], Gendelman et al [29], e King e Vakakis [39]. Em Lacarbonara et al [41], [42], encontra-se o estudo das ressonâncias internas para estruturas unidimensionais e estruturas abatidas.

As bases do desenvolvimento das equações não-lineares de movimento utilizadas neste trabalho podem ser encontradas em Mazzilli [48] (1990) e Brasil e Mazzilli [15]. Mazzilli, Soares e Baracho Neto publicam, em 2001, [49] artigo sobre redução de modelos discretizados pelo método dos elementos finitos utilizando modos não-lineares de vibração.

Em 2000, Soares et al [87] desenvolvem o cálculo dos modos não-lineares por meio de software de cálculo. Abordam-se sistemas formados por barras e discretizados pelo método dos elementos finitos. Utiliza-se a abordagem por variedades invariantes. Uma explanação mais extensa sobre o tópico pode ser apreciada em [86] (1998).

Baracho Neto [6] também aborda estruturas compostas por barras discretizadas por elementos finitos, porém determinando os modos normais e os multi-modos pelo método das múltiplas escalas. Encontram-se soluções temporais, recuperando-se posteriormente as relações modais, ou seja, as variedades invariantes. Outros trabalhos de Baracho Neto [5],[7] e [8] exploram modos normais não-lineares 
e multi-modos não-lineares com ressonâncias internas 1:3 e 1:2 utilizando o método das múltiplas escalas. Boivin et al [11], [12] e [13] também aborda ressonâncias internas e vibrações forçadas.

Pesce et al [70], em 1999, publicam trabalho sobre a determinação dos autovalores de risers em águas profundas. $\mathrm{O}$ trabalho apresenta uma solução analítica fechada. São abordados sistemas linearizados considerando a rigidez geométrica dos risers.

Pesce e Martins [71] em 2005 apresentam modelo de interação fluido estrutura. Trata-se de modelo numérico computacional para a determinação da dinâmica de risers.

Um ano depois, 2006, Pesce e Martins [72] publicam trabalho sobre a dinâmica local na região do TDP (Touch Down Point) relacionando esta última com a dinâmica global do riser. Ainda abordam a determinação dos modos de vibração e suas relações com as excitações provenientes de vibrações induzidas por vórtices VIV.

Em 1989, Martins [47] publica trabalho sobre o amortecimento ativo para redução de vibrações induzidas por vórtices (VIV) em risers. Silveira et al [84] investiga a tensão efetiva em risers submetidos à VIV.

Em Delft, Keber et al [35], 2006, apresentam trabalho aplicando a análise modal não-linear ao estudo da dinâmica de risers. As vibrações são induzidas por vórtices (VIV) e modos não-lineares são calculados por variedades invariantes.

Em 2007, trabalho apresentado por Keber et al [36] discute a dinâmica de risers considerando não-linearidades geométricas fracas e excitados por correntes oceânicas. Como seqüência deste trabalho, Keber et al [37] publicam estudo sobre a interação fluido-estrutura em risers excitados por vórtices oriundos de corrente oceânica. Na seqüência dos trabalhos, Keber [38] realiza estudo sobre a influência da tensão inicial em risers, com foco em como a protensão inicial atenua ou aumenta a amplitude da resposta temporal. 
Newberry et al [64] também propõem contribuições interessantes ao estudo de cabos submersos com ressonância interna 1:1.

Em Parra [67] e Blevins [10] valiosos desenvolvimentos teóricos e resultados experimentais podem ser apreciados no estudo das vibrações induzidas por vórtices $(V I V)$ em risers rígidos acoplados a um sistema de molas. Ainda em Blevins encontram-se resultados também para risers flexíveis.

Kuiper [40] publica resultado interessante, ainda não totalmente compreendido, sobre vibrações em water intake risers (risers para tomada de água) que sugerem a possível existência de ressonâncias internas em tais aplicações.

Mazzilli [50], 2007, publica abordagem analítica para o cáculo dos modos não-lineares em barras pré-tensionadas comparando as respostas analíticas com respostas obtidas via MEF e variedades invariantes. A determinação analítica dos modos não-lineares é feita através do método das múltiplas escalas. A extensão deste trabalho é apresentada em [51], com o cálculo de multi-modo, além dos modos normais, pela via analítica e utilizando $\mathrm{MEF}$, ambos utilizando o método das múltiplas escalas. Esse multi-modo acopla o primeiro e o terceiro modos de vibração que interagem fortemente devido à ressonância interna.

Em 1996, González Lima [30] apresenta trabalho sobre a translação de viga flexível em tempo mínimo. Neste estudo, valiosos conceitos relativos à utilização do controle ativo ótimo são detalhados. Adicionalmente, um modelo físico é construído e utilizado para obtenção de resultados experimentais que calibram o modelo teórico. Neste trabalho fica clara a construção do observador e suas implicações na dinâmica do sistema. O observador de estados utilizado neste texto é o observador de Luenberger [44]. Em Chang [17] e Ogata [65] encontram-se os desenvolvimentos relativos ao regulador quadrático linear (Linear Quadratic Regulator - LQR) adotados neste texto.

Preumont, [73] e [74] (2002, 1997), publica amplo trabalho em controle ativo de estruturas. Nesses trabalhos nota-se forte ênfase no estudo de cabos e estruturas 
associadas a estes. Diversas abordagens são apresentas utilizando sistemas SISO e MIMO com alocação de pólos utilizando regulador quadrático ótimo.

Gattulli ([24], [25], [26], [27] e [28]) publica extenso estudo sobre dinâmica não-linear, especialmente, de cabos. Também aborda o controle da vibração de cabos associados a outros sistemas estruturais. Tais controles podem ser passivos e ativos. Para ambos, resultados experimentais são gerados realimentando os modelos matemáticos. Srinil et al [88], [89] e [90] contribuem com estudo de cabos com ressonância interna.

Em Fujino et al [21], [22] encontram-se estudos interessantes com relação ao controle ativo de cabos submetidos à vibrações forçadas. Bossens e Preumont [14], Achkire e Preumont [1], [2] abordam o problema de cabos em pontos e seus sistemas de medição de vibrações oriundas de esforços aerodinâmicos (Fung [23]).

\subsection{Organização deste Texto}

O capítulo 2 aborda conceitos relativos a modos não-lineares de vibração. Inicia-se o capítulo com modos não-lineares normais e variedades invariantes. Em seguida disserta-se sobre o método dos elementos finitos e o elemento não-linear utilizado na discretização dos risers. Posteriormente, ainda no mesmo capítulo, procede-se ao desenvolvimento dos multi-modos não-lineares. Discutem-se ainda as implementações feitas para o cálculo de modos não-lineares de risers. Ressalta-se que o software aqui utilizado somente constrói modelos bidimensionais, ou seja, modelos planos.

O capítulo 3 trata de modos não-lineares de risers retos. São analisados risers sem peso próprio, com peso próprio e com corrente. Além disso, são determinados os multi-modos dos exemplos sem e com peso próprio. Comparações são feitas para diferentes amplitudes iniciais e os resultados são comparados entre si. Para o riser com corrente, é estudada sua dinâmica quando são considerados VIV's (vibrações induzidas por vórtices). 
No capítulo 4, determinam-se os modos não-lineares normais para risers em catenária (SCR's). Relações da freqüência com amplitude são abordadas, assim como as mudanças nas formais modais quando os efeitos não-lineares são considerados. Também se discute a capacidade de mesmo um único modo não-linear de vibração capturar influências de modos superiores. Além disso, para o SCR de extremidades fixas ou fixa-ancorada, são estudadas as vibrações induzidas por vórtices.

No capítulo 5, são introduzidos sistemas de controle ativo para os risers do capítulo 3. Comparam-se resultados com e sem controle: amplitudes, freqüências, respostas no tempo e diagramas de fase. Também se abordam os níveis de tensão e taxa de amortecimento para tais sistemas de controle. Estudam-se sistemas de controle ativo ótimo, as vibrações induzidas por vórtice para o riser reto com peso próprio e o $S C R$ com extremos fixos.

Por fim, o capítulo 6 traz as conclusões e trabalhos futuros. Fechando o texto encontram-se as referências usadas nesta pesquisa. 


\section{Capítulo 2}

\section{Modos Não-Lineares de Vibração}

Este capítulo tem por objetivo a conceituação e o estudo do procedimento de cálculo para modos não-lineares. Apresenta-se a abordagem por variedades invariantes para a determinação dos modos não-lineares normais. Primeiramente, aplica-se esse conceito para os sistemas lineares. Posteriormente, o método é estendido para os sistemas não-lineares. O sistema não-linear de segunda ordem com $n$ equações diferenciais é transformado em um sistema de primeira com $2 n$ equações diferenciais. A partir do sistema de primeira ordem determinam-se as relações modais e a equação não-linear do oscilador modal.

Discute-se, também, a discretização de sistemas estruturais pelo método dos elementos finitos, abordando as não-linearidades geométricas consideradas na formulação do elemento. Utiliza-se elemento plano de viga de eixo reto baseado na teoria de Bernoulli-Euler considerando em sua formulação não-linearidades geométricas. No final da seção, apresentam-se os desenvolvimentos matemáticos implementados no software de cálculo para a consideração de risers. Tais alterações tornam o software capaz de lidar com as tensões internas dos risers oriundas da configuração estática dessas estruturas.

Por fim, apresenta-se a determinação dos multi-modos. Estes últimos são calculados utilizando-se o método das múltiplas escalas.

Para o cáculo dos modos não-lineares normais foi gerado um software, em FORTRAN, chamado modonlr. Este software é uma versão atualizada do modonl, ver [86] e [77], que recebeu aprimoramentos para poder processar risers. Portanto, o modonlr é um subproduto deste trabalho. Para o cálculo dos multi-modos nãolineares, utilizou-se um software gerado por Baracho Neto [6]. Este capítulo apresenta as teorias e os modelos matemáticos utilizados na elaboração dos programas. Ambos os programas tratam, apenas, de modelos planos e vibrações livres. 


\subsection{Variedades Invariantes}

\subsubsection{Variedades Invariantes em Sistemas Lineares}

Considere-se um sistema estrutural linear, com $n$ graus de liberdade, que possua amortecimento proporcional ou não-proporcional. A equação matricial deste sistema pode ser escrita na forma:

$$
\mathbf{M} \ddot{\mathbf{x}}+\mathbf{C} \dot{\mathbf{x}}+\mathbf{K} \mathbf{x}=\mathbf{0}
$$

onde $\mathbf{M}$ é a matriz de massa, $\mathbf{C}$ é a matriz de amortecimento e $\mathbf{K}$ é a matriz de rigidez, todas quadradas de ordem $n$.

O equação 2.1 pode ser escrita na forma:

$$
\dot{\mathbf{z}}=\mathbf{T} \mathbf{z} .
$$

onde $\mathbf{z}=\left[\begin{array}{l}\mathbf{x} \\ \mathbf{y}\end{array}\right], \mathbf{y}=\dot{\mathbf{x}}, \mathbf{T}=\left[\begin{array}{cc}\mathbf{0} & \mathbf{I} \\ -\mathbf{M}^{-1} \mathbf{K} & -\mathbf{M}^{-1} \mathbf{C}\end{array}\right]$

Para o sistema 2.2 os deslocamentos generalizados para um determinado modo $r$ (ver [86]) pode ser escrito como função das variáveis modais na forma:

$$
\mathbf{z}^{r}=\mathbf{N}^{r} \mathbf{w}
$$

onde, $\mathbf{N}=\left\lfloor\begin{array}{ll}\mathbf{N}_{1}^{r} & \mathbf{N}_{2}^{r}\end{array}\right], \mathbf{N}_{1}^{r}$ e $\mathbf{N}_{2}^{r}$ são a parte real e a parte imaginária do autovetor $r$ da matriz $\mathbf{T} ; \mathbf{w}=\left[\begin{array}{l}u(t) \\ v(t)\end{array}\right]$, sendo $u(t)$ e $v(t)$ o deslocamento modal e a velocidade modal, respectivamente, com $v=\dot{u}$.

A equação 2.3 mostra que o vetor de deslocamentos e velocidades generalizados do r-ésimo modo $\mathbf{z}^{\mathrm{r}}$ é uma combinação linear de $\mathbf{N}_{1}^{\mathrm{r}}$ e $\mathbf{N}_{2}^{\mathrm{r}}$. Nota-se, 
portanto, que o movimento modal no espaço de fase de dimensão $2 n$, está restrito ao subespaço $S^{r}$ gerado por $\mathbf{N}_{1}^{r}$ e $\mathbf{N}_{2}^{r}$, isto é, dar uma condição inicial que pertença a $S^{r}$ faz com que todo o movimento se desenvolva neste subespaço. Desta forma, entendese que $\mathrm{S}^{\mathrm{r}}$ é um subespaço invariante ao fluxo.

Vale notar que $\mathbf{N}_{1}^{\mathrm{r}}$ e $\mathbf{N}_{2}^{\mathrm{r}}$ são vetores linearmente independentes e, portanto, distintos entre si. Logo, $\mathbf{N}_{1}^{\mathrm{r}}$ e $\mathbf{N}_{2}^{\mathrm{r}}$ podem apresentar deslocamentos generalizados distintos. Desta forma, $\mathbf{N}_{1}^{\mathrm{r}}$ e $\mathbf{N}_{2}^{\mathrm{r}}$ podem representar formas modais diferentes. As variáveis $u(t)$ e $v(t)$ são, como já mencionado anteriormente, respectivamente, o deslocamento modal e a velocidade modal. Portanto, quando $v(t)$ é nulo, $u(t)$ é diferente de zero sendo a forma modal definida pelo vetor $\mathbf{N}_{1}^{\mathrm{r}}$. Quando $u(t)$ é nulo, $v(t)$ é diferente de zero e a forma modal é definida pelo vetor $\mathbf{N}_{2}^{\mathrm{r}}$. Devido a esta alternância de formas modais, o movimento observado pode ser uma onda nãoestacionária, no caso de amortecimento não-proporcional, ( $\operatorname{se} \mathbf{N}_{2}^{r} \neq 0$ ), onde os deslocamentos generalizados podem não se anular no mesmo instante e, também, podem não atingir seus valores máximos no mesmo instante.

$\mathrm{O}$ subespaço $\mathrm{S}^{\mathrm{r}}$ pode ser entendido como uma variedade, do ponto de vista topológico [86]. Desta forma, entende-se que o subespaço $\mathrm{S}^{\mathrm{r}}$ é uma variedade invariante do sistema dinâmico. Conclui-se, portanto, que a solução modal para um sistema linear com amortecimento não-proporcional é um movimento que se processa numa variedade invariante bidimensional no espaço de fase [79]. Vale notar que a definição clássica de modos de vibração não se aplica a sistemas lineares com amortecimento não-proporcional.

Nos sistemas lineares, as variedades invariantes do sistema dinâmico são planos do espaço de fase que contêm o ponto de equilíbrio. Assim, todos os deslocamentos e velocidades generalizados podem ser escritos como uma função linear das variáveis modais, que são um deslocamento generalizado e sua respectiva velocidade. Partindo deste fato, é possível propor um procedimento diferente para o cálculo das soluções modais. Este novo procedimento consiste em determinar, primeiramente, as funções que relacionam deslocamentos e velocidades generalizados 
com as variáveis modais. Estas funções são ditas relações modais. Na verdade, tratase de determinar as variedades invariantes do sistema dinâmico. Uma vez encontradas as variedades, é possível definir a dinâmica do sistema através da equação do oscilador modal equivalente. Este procedimento é uma generalização do método geométrico de Rosenberg [75].

Sendo assim, as variáveis modais são escolhidas na seguinte forma:

$$
u(t)=x_{k}(t)
$$

e

$$
v(t)=y_{k}(t)=\dot{x}_{k}(t)
$$

sendo $\mathrm{u}(\mathrm{t})$ e $\mathrm{v}(\mathrm{t})$ as variáveis modais e o índice $k$ refere-se ao grau de liberdade escolhido para definição das variáveis modais.

Considerem-se dois vetores linearmente independentes $\mathbf{N}_{1}$ e $\mathbf{N}_{2}$ de dimensão $2 n$, que definem uma variedade invariante no espaço de fase. Pode-se escrever,

$$
\mathbf{z}=\mathbf{N} \mathbf{w}
$$

onde 


$$
\mathbf{N}=\left[\begin{array}{cc}
a_{11} & a_{21} \\
\vdots & \vdots \\
1 & 0 \\
\vdots & \vdots \\
a_{1 n} & a_{2 n} \\
b_{11} & b_{21} \\
\vdots & \vdots \\
0 & 1 \\
\vdots & \vdots \\
b_{1 n} & b_{2 n}
\end{array}\right]=\left[\begin{array}{ll}
\mathbf{N}_{1} & \mathbf{N}_{2}
\end{array}\right]
$$

$\mathrm{e}$

$$
\mathbf{w}=\left[\begin{array}{l}
u \\
v
\end{array}\right]=\left[\begin{array}{c}
x_{k} \\
\dot{x}_{k}
\end{array}\right]=\left[\begin{array}{l}
x_{k} \\
y_{k}
\end{array}\right] .
$$

Substituindo 2.6 em 2.2,

$$
\mathbf{N} \dot{\mathbf{w}}=\mathbf{T} \mathbf{N} \mathbf{w} .
$$

Observando a equação 2.9 , nota-se que a dependência com relação ao tempo está guardada nas variáveis $\mathbf{w}$ e $\dot{\mathbf{w}}$. Para eliminar-se a dependência do tempo, iniciase por se escrever $\dot{\mathbf{w}}$ na forma,

$$
\dot{\mathbf{w}}=\left[\begin{array}{c}
\dot{\mathrm{u}} \\
\dot{\mathrm{v}}
\end{array}\right]=\left[\begin{array}{c}
\mathrm{v} \\
\dot{\mathrm{v}}
\end{array}\right]=\left[\begin{array}{c}
\dot{\mathrm{z}}_{\mathrm{k}} \\
\dot{\mathrm{z}}_{\mathrm{n}+\mathrm{k}}
\end{array}\right] .
$$

Substituindo 2.6 à direita da igualdade em 2.2 vem,

$$
\dot{\mathbf{z}}=\mathbf{T} \mathbf{N} \mathbf{w} .
$$

A expressão 2.11 pode ser escrita de forma mais explícita como, 


$$
\left[\begin{array}{c}
\dot{z}_{1} \\
\vdots \\
\dot{z}_{k} \\
\vdots \\
\dot{z}_{n} \\
\vdots \\
\dot{z}_{n+k} \\
\vdots \\
\dot{z}_{2 n}
\end{array}\right]=\left[\begin{array}{cccc}
T_{11} & \cdots & \cdots & T_{1 n} \\
\vdots & & & \vdots \\
\vdots & & & \vdots \\
\vdots & & & \vdots \\
\vdots & & & \vdots \\
\vdots & & & \vdots \\
T_{n+k, 1} & \cdots & \cdots & T_{n+k, 2 n} \\
\vdots & & & \vdots \\
T_{2 n, 1} & \cdots & \cdots & T_{2 n, 2 n}
\end{array}\right]\left[\begin{array}{cc}
a_{11} & a_{12} \\
\vdots & \vdots \\
\vdots & \vdots \\
\vdots & \vdots \\
\vdots & \vdots \\
\vdots & \vdots \\
b_{2 n, 1} & b_{2 n, 2 n}
\end{array}\right]\left[\begin{array}{c}
u \\
v
\end{array}\right] .
$$

De 2.12, utilizando notação indicial, vem,

$$
\dot{z}_{n+k}=\dot{v}=T_{n+k, i}\left[n_{i 1} u+n_{i 2} v\right] .
$$

onde, $n_{i 1}$ e $n_{i 2}$ são os elementos da matriz $\mathbf{N}$.

Substituindo 2.13 em 2.10 tem-se,

$$
\dot{\mathbf{w}}=\left[\begin{array}{c}
\dot{\mathrm{u}} \\
\dot{\mathrm{v}}
\end{array}\right]=\left[\begin{array}{c}
\mathrm{v} \\
\dot{\mathrm{v}}
\end{array}\right]=\left[\begin{array}{c}
\dot{\mathrm{z}}_{\mathrm{k}} \\
\dot{\mathrm{z}}_{\mathrm{n}+\mathrm{k}}
\end{array}\right]=\left[\begin{array}{cc}
0 & 1 \\
\mathrm{~T}_{\mathrm{n}+\mathrm{k}, \mathrm{i}} \mathrm{n}_{\mathrm{i} 1} & \mathrm{~T}_{\mathrm{n}+\mathrm{k}, \mathrm{i}} \mathrm{n}_{\mathrm{i} 2}
\end{array}\right]\left[\begin{array}{c}
\mathrm{u} \\
\mathrm{v}
\end{array}\right]=\mathbf{Q} \mathbf{w},
$$

onde,

$$
\mathbf{Q}=\left[\begin{array}{cc}
0 & 1 \\
\mathrm{~T}_{\mathrm{n}+\mathrm{k}, \mathrm{i}} \mathrm{n}_{\mathrm{i} 1} & \mathrm{~T}_{\mathrm{n}+\mathrm{k}, \mathrm{i}} \mathrm{n}_{\mathrm{i} 2}
\end{array}\right] .
$$

Substituindo 2.14 em 2.9,

$$
\mathbf{N} \mathbf{Q} \mathbf{w}=\mathbf{T} \mathbf{N} \mathbf{w},
$$

e, portanto, dada a arbitrariedade de $\mathbf{w}$,

$$
\mathbf{N Q}=\mathbf{T} \mathbf{N}
$$


Após escrever $\dot{\mathbf{w}}$ como função de $\mathbf{w}$, e substituir na equação 2.9, obtém-se a expressão 2.17 que independe do tempo. Na verdade as variáveis em questão são apenas aquelas que descrevem as relações modais, isto é, são os elementos da matriz N . O problema agora se torna puramente geométrico, sendo que a determinação de $\mathbf{N}$ define a variedade invariante na qual se desenvolve o movimento.

Explicitando os elementos de 2.17, vem

$\left[\begin{array}{cc}\mathrm{n}_{11} & \mathrm{n}_{12} \\ \vdots & \vdots \\ 1 & 0 \\ \vdots & \vdots \\ \vdots & \vdots \\ \vdots & \vdots \\ 0 & 1 \\ \vdots & \vdots \\ \mathrm{n}_{2 \mathrm{n}, 1} & \mathrm{n}_{2 \mathrm{n}, 2 \mathrm{n}}\end{array}\right]\left[\begin{array}{cc}0 & 1 \\ \mathrm{~T}_{\mathrm{n}+\mathrm{k}, \mathrm{i}} \mathrm{n}_{\mathrm{i} 1} & \mathrm{~T}_{\mathrm{n}+\mathrm{k}, \mathrm{i}} \mathrm{n}_{\mathrm{i} 2}\end{array}\right]=\left[\begin{array}{cccc}\mathrm{T}_{11} & \cdots & \cdots & \mathrm{T}_{1 \mathrm{n}} \\ \vdots & & & \vdots \\ \mathrm{T}_{\mathrm{k} 1} & \cdots & \cdots & \mathrm{T}_{\mathrm{k}, 2 \mathrm{n}} \\ \vdots & & & \vdots \\ \vdots & & & \vdots \\ \vdots & & & \vdots \\ \mathrm{T}_{\mathrm{n}+\mathrm{k}, 1} & \cdots & \cdots & \mathrm{T}_{\mathrm{n}+\mathrm{k}, 2 \mathrm{n}} \\ \vdots & & & \vdots \\ \mathrm{T}_{2 \mathrm{n}, 1} & \cdots & \cdots & \mathrm{T}_{2 \mathrm{n}, 2 \mathrm{n}}\end{array}\right]\left[\begin{array}{cc}\mathrm{n}_{11} & \mathrm{n}_{12} \\ \vdots & \vdots \\ 1 & 0 \\ \vdots & \vdots \\ \vdots & \vdots \\ \vdots & \vdots \\ 0 & 1 \\ \mathrm{n}_{2 \mathrm{n}, 1} & \mathrm{n}_{2 \mathrm{n}, 2 \mathrm{n}}\end{array}\right] .18$

Lembrando 2.2, reescreve-se 2.18 como

$$
\left[\begin{array}{cc}
\mathrm{n}_{11} & \mathrm{n}_{12} \\
\vdots & \vdots \\
1 & 0 \\
\vdots & \vdots \\
\vdots & \vdots \\
\vdots & \vdots \\
0 & 1 \\
\vdots & \vdots \\
\mathrm{n}_{2 \mathrm{n}, 1} & \mathrm{n}_{2 \mathrm{n}, 2 \mathrm{n}}
\end{array}\right]\left[\begin{array}{cc}
0 & 1 \\
\mathrm{~T}_{\mathrm{n}+\mathrm{k}, \mathrm{i}} \mathrm{n}_{\mathrm{i} 1} & \mathrm{~T}_{\mathrm{n}+\mathrm{k}, \mathrm{i}} \mathrm{n}_{\mathrm{i} 2}
\end{array}\right]=\left[\begin{array}{cccccccc}
0 & \cdots & \cdots & 0 & 1 & \cdots & \cdots & 0 \\
\vdots & \cdots & \cdots & \vdots & \vdots & \ddots & & \vdots \\
\vdots & \cdots & \cdots & \vdots & \vdots & & \ddots & \vdots \\
0 & \cdots & \cdots & 0 & 0 & \cdots & \cdots & 1 \\
\mathrm{~T}_{\mathrm{n}+1,1} & \cdots & \cdots & \cdots & \cdots & \cdots & \cdots & \mathrm{T}_{\mathrm{n}+1,2 \mathrm{n}} \\
\vdots & \cdots & \cdots & \cdots & \cdots & \cdots & \cdots & \vdots \\
\mathrm{T}_{\mathrm{n}+\mathrm{k}, 1} & \cdots & \cdots & \cdots & \cdots & \cdots & \cdots & \mathrm{T}_{\mathrm{n}+\mathrm{k}, 2 \mathrm{n}} \\
\vdots & \cdots & \cdots & \cdots & \cdots & \cdots & \cdots & \vdots \\
\mathrm{T}_{2 \mathrm{n}, 1} & \cdots & \cdots & \cdots & \cdots & \cdots & \cdots & \mathrm{T}_{2 \mathrm{n}, 2 \mathrm{n}}
\end{array}\right]\left[\begin{array}{cc}
\mathrm{n}_{11} & \mathrm{n}_{12} \\
\vdots & \vdots \\
1 & 0 \\
\vdots & \vdots \\
\vdots & \vdots \\
\vdots & \vdots \\
0 & 1 \\
\vdots & \vdots \\
\mathrm{n}_{2 \mathrm{n}, 1} & \mathrm{n}_{2 \mathrm{n}, 2 \mathrm{n}}
\end{array}\right] .
$$

A partir de 2.19 nota-se que $\mathbf{N} \mathbf{Q}$ é uma matriz $2 n \times 2$, assim como $\mathbf{T} \mathbf{N}$. Logo, se numa primeira leitura conclui-se que sejam $4 n$ equações não-lineares e independentes, deve-se observar que as equações correspondentes às linhas $k$ e $n+k$ são previamente satisfeitas, e, desta maneira, restam $4 n-4$ equações. 
Vale lembrar que, para um sistema estrutural com $n$ graus de liberdade, existem $n$ modos de vibração e que, portanto, teremos $n$ subespaços $S^{r}$ que representam as variedades invariantes do problema. No entanto, cada subespaço $\mathrm{S}^{\mathrm{r}}$ é gerado a partir do conhecimento de dois vetores linearmente independentes $\mathbf{N}_{1}^{\mathrm{r}}$ e $\mathbf{N}_{2}^{\mathrm{r}}$, que são componentes de $\mathbf{N}^{\mathrm{r}}$. Assim, o sistema 2.17 deve apresentar $n$ soluções reais distintas para $\mathbf{N}$, correspondendo às $n$ variedades invariantes.

Uma vez determinadas as relações modais segue-se a determinação do oscilador modal equivalente. Particularizando 2.11 para o r-ésimo modo,

$$
\dot{\mathbf{z}}^{\mathrm{r}}=\mathbf{T} \mathbf{N}^{\mathrm{r}} \mathbf{w}^{\mathrm{r}}
$$

e observando que, como mostra 2.13,

$$
\ddot{\mathrm{u}}^{\mathrm{r}}=\dot{\mathrm{v}}^{\mathrm{r}}=\dot{\mathrm{z}}_{\mathrm{n}+\mathrm{k}}^{\mathrm{r}}=\mathrm{T}_{\mathrm{n}+\mathrm{k}, \mathrm{i}}\left[\mathrm{n}_{\mathrm{i} 1}^{\mathrm{r}} \mathrm{u}^{\mathrm{r}}+\mathrm{n}_{\mathrm{i} 2}^{\mathrm{r}} \mathrm{v}^{\mathrm{r}}\right] .
$$

A repetição de um índice $i$ denota a soma em $i$ até $n$ (notação indicial). Ao longo deste texto será utilizada esta notação, excetuando os casos onde, explicitamente, seja dito o contrário.

Obtém-se,

$$
\ddot{u}^{r}+2 \xi^{r} \omega^{r} \dot{u}^{r}+\left(\omega^{r}\right)^{2} u^{r}=0
$$

onde

$$
\omega^{\mathrm{r}}=\sqrt{-\mathrm{T}_{\mathrm{n}+\mathrm{k}, \mathrm{i}} \mathrm{n}_{\mathrm{i} 1}^{\mathrm{r}}}
$$

é a freqüência natural do modo $r$, e 


$$
\xi^{\mathrm{r}}=\frac{-\mathrm{T}_{\mathrm{n}+\mathrm{k}, \mathrm{i}} \mathrm{n}_{\mathrm{i} 2}^{\mathrm{r}}}{2 \omega^{\mathrm{r}}}
$$

é a taxa de amortecimento do modo em questão.

A solução do oscilador modal 2.22 é dada por:

$$
u^{r}(t)=e^{-\xi^{r} \omega^{r} t} \rho^{r} \cos \left(\omega_{d}^{r} t+\varphi^{r}\right)
$$

onde

$$
\omega_{d}^{r}=\omega^{r} \sqrt{1-\xi_{r}^{2}}
$$

é a freqüência amortecida do $r$-ésimo modo, e $\rho^{r}$ e $\varphi^{r}$ são constantes que dependem das condições iniciais.

Esta abordagem visa a encontrar primeiramente as relações modais e depois as equações que descrevem a dinâmica do modo para compor o movimento total.

A solução de 2.17, pode demandar um considerável esforço computacional. Logo, para os modos lineares de vibração, uma abordagem através de um problema de autovalores, pode representar um ganho, tendo em vista a existência de rotinas eficientes no tratamento de tais problemas, mesmo sendo $\mathbf{T}$ não simétrica.

Porém, esta visão geométrica será importante para a definição dos modos nãolineares, uma das extensões do conceito aqui apresentado.

\subsubsection{Variedades Invariantes em Sistemas Não-Lineares}

Neste trabalho, considera-se a definição proposta por Shaw e Pierre [79]: "Modo não-linear de vibração é um movimento não-forçado que se desenvolve em uma variedade invariante bidimensional no espaço de fase do sistema; essa variedade 
contém um ponto de equilíbrio estável e, nesse ponto, é tangente a um plano que corresponde a um autoespaço do sistema linearizado em torno do equilíbrio".

Pode-se entender modos não-lineares de vibração como "uma extensão natural do conceito de modos de vibração para sistemas não lineares". Em sistemas lineares, os deslocamentos e velocidades generalizados podem ser escritos como uma combinação linear de apenas um deslocamento generalizado e sua respectiva velocidade e, desta forma, o movimento fica restrito a um subespaço bidimensional e plano $\mathrm{S}^{\mathrm{r}}$ do espaço de fase de dimensão $2 n$. Para sistemas não-lineares, os deslocamentos e velocidades são escritos como função de um deslocamento generalizado e sua respectiva velocidade, porém estas funções são geralmente nãolineares. Em conseqüência, o subespaço $S^{r}$ mantém-se bidimensional, porém trata-se agora de uma superfície no espaço de fase, em geral, não-plana.

Para uma compreensão melhor, supõe-se o seguinte conjunto de equações diferenciais de movimento de segunda ordem de um sistema não-linear autônomo,

$$
\ddot{x}_{\mathrm{i}}=\mathrm{f}_{\mathrm{i}}\left(\mathrm{x}_{1}, \ldots, \mathrm{x}_{\mathrm{n}}, \mathrm{y}_{1}, \ldots, \mathrm{y}_{\mathrm{n}}\right) \text {, }
$$

ou, na forma de um sistema de primeira ordem:

$$
\left\{\begin{array}{l}
\dot{x}_{i}=\dot{y}_{i} \\
\dot{y}_{i}=f_{i}\left(x_{1}, \ldots, x_{n}, y_{1}, \ldots, y_{n}\right)
\end{array},\right.
$$

onde $\mathrm{i}=1, \ldots, \mathrm{n}$ e $\mathrm{f}_{\mathrm{i}}$ são funções de classe $\mathrm{C}^{1}$, sujeitas às seguintes condições iniciais

$$
f_{i}(0, \ldots, 0,0, \ldots, 0)=0
$$

$\operatorname{com} \mathrm{i}=1, \ldots, \mathrm{n}$.

$\mathrm{Na}$ forma matricial, tem-se 
onde,

$$
\begin{gathered}
\dot{\mathbf{z}}=\left[\begin{array}{c}
\dot{\mathrm{z}}_{1} \\
\vdots \\
\dot{\mathrm{z}}_{\mathrm{n}} \\
\dot{\mathrm{z}}_{\mathrm{n}+1} \\
\vdots \\
\dot{\mathrm{z}}_{2 \mathrm{n}}
\end{array}\right]=\left[\begin{array}{c}
\dot{\mathrm{x}}_{1} \\
\vdots \\
\dot{\mathrm{x}}_{\mathrm{n}} \\
\dot{\mathrm{y}}_{1} \\
\vdots \\
\dot{\mathrm{y}}_{\mathrm{n}}
\end{array}\right] \mathrm{e} \\
\mathbf{F}\left(\mathrm{z}_{1}, \ldots, \mathrm{z}_{2 \mathrm{n}}\right)=\left[\begin{array}{c}
\mathrm{z}_{\mathrm{n}+1} \\
\vdots \\
\mathrm{z}_{2 \mathrm{n}} \\
\mathrm{f}_{1}\left(\mathrm{z}_{1}, \ldots, \mathrm{z}_{2 \mathrm{n}}\right) \\
\vdots \\
\mathrm{f}_{\mathrm{n}}\left(\mathrm{z}_{1}, \ldots, \mathrm{z}_{2 \mathrm{n}}\right)
\end{array}\right] .
\end{gathered}
$$

O correspondente sistema linearizado de 2.30 pode ser determinado a partir de

$$
\dot{\mathbf{z}}=\frac{\partial}{\partial \mathbf{z}} \mathbf{F}(\mathbf{0}) \mathbf{z}
$$

Sendo

$$
\mathbf{T}=\frac{\partial}{\partial \mathbf{z}} \mathbf{F}(\mathbf{0})
$$

vem

$$
\dot{\mathbf{z}}=\mathbf{T} \mathbf{z} \text {. }
$$


Estendendo-se o conceito utilizado para os modos lineares, escrevem-se os deslocamentos e velocidades como funções de apenas um deslocamento generalizado e sua respectiva velocidade. Para tanto, escolhem-se:

$$
u(t)=x_{k}(t)
$$

e

$$
v(t)=y_{k}(t)=\dot{x}_{k}(t)
$$

sendo as variáveis modais.

Desta forma, durante um movimento modal qualquer deslocamento ou velocidade é uma função de $u$ e $v$,

$$
\begin{aligned}
& \mathrm{x}_{\mathrm{i}}=\mathrm{X}_{\mathrm{i}}(\mathrm{u}, \mathrm{v}), \\
& \mathrm{y}_{\mathrm{i}}=\mathrm{Y}_{\mathrm{i}}(\mathrm{u}, \mathrm{v}),
\end{aligned}
$$

$\operatorname{com} \mathrm{i}=1, \ldots, \mathrm{n}$.

e, em especial,

$$
\begin{aligned}
& \mathrm{x}_{\mathrm{k}}=\mathrm{X}_{\mathrm{k}}(\mathrm{u}, \mathrm{v})=\mathrm{u}, \\
& \mathrm{y}_{\mathrm{k}}=\mathrm{Y}_{\mathrm{k}}(\mathrm{u}, \mathrm{v})=\mathrm{v} .
\end{aligned}
$$

Substituindo as expressões 2.38 e 2.39 em 2.28 obtêm-se

$$
\begin{aligned}
& \dot{\mathrm{x}}_{\mathrm{i}}=\mathrm{y}_{\mathrm{i}}=\mathrm{Y}_{\mathrm{i}}(\mathrm{u}, \mathrm{v}) \\
& \dot{\mathrm{y}}_{\mathrm{i}}=\mathrm{f}_{\mathrm{i}}\left(\mathrm{x}_{1}, \ldots, \mathrm{x}_{\mathrm{n}}, \mathrm{y}_{1}, \ldots, \mathrm{y}_{\mathrm{n}}\right)=\mathrm{f}_{\mathrm{i}}\left(\mathrm{X}_{1}(\mathrm{u}, \mathrm{v}), \ldots, \mathrm{X}_{\mathrm{n}}(\mathrm{u}, \mathrm{v}), \mathrm{Y}_{1}(\mathrm{u}, \mathrm{v}), \ldots, \mathrm{Y}_{\mathrm{n}}(\mathrm{u}, \mathrm{v})\right)
\end{aligned} .
$$


Em notação matricial tem-se,

$$
\begin{aligned}
& \mathbf{x}=\mathbf{X}(\mathrm{u}, \mathrm{v}), \\
& \mathbf{y}=\mathbf{Y}(\mathrm{u}, \mathrm{v}),
\end{aligned}
$$

onde

$$
\mathbf{x}=\left[\begin{array}{c}
\mathrm{x}_{1} \\
\vdots \\
\mathrm{x}_{\mathrm{n}}
\end{array}\right], \quad \mathbf{X}(\mathrm{u}, \mathrm{v})=\left[\begin{array}{c}
\mathrm{X}_{1}(\mathrm{u}, \mathrm{v}) \\
\vdots \\
\mathrm{X}_{\mathrm{n}}(\mathrm{u}, \mathrm{v})
\end{array}\right], \quad \mathbf{y}=\left[\begin{array}{c}
\mathrm{y}_{1} \\
\vdots \\
\mathrm{y}_{\mathrm{n}}
\end{array}\right], \quad \text { e } \quad \mathbf{Y}(\mathrm{u}, \mathrm{v})=\left[\begin{array}{c}
\mathrm{Y}_{1}(\mathrm{u}, \mathrm{v}) \\
\vdots \\
\mathrm{Y}_{\mathrm{n}}(\mathrm{u}, \mathrm{v})
\end{array}\right]
$$

Utilizando 2.43 e 2.44 pode-se escrever $\mathbf{z}$ na forma,

$$
\mathbf{z}=\left[\begin{array}{l}
\mathbf{x} \\
\mathbf{y}
\end{array}\right]=\left[\begin{array}{l}
\mathbf{X}(\mathrm{u}, \mathrm{v}) \\
\mathbf{Y}(\mathrm{u}, \mathrm{v})
\end{array}\right]
$$

Deseja-se eliminar a dependência do tempo na expressão 2.30. Portanto, desenvolve-se a derivada no tempo de $\mathbf{z}$ na forma,

$$
\dot{\mathbf{z}}=\frac{\mathrm{d}}{\mathrm{dt}} \mathbf{z}=\frac{\partial \mathbf{z}}{\partial \mathbf{w}} \frac{\mathrm{d} \mathbf{w}}{\mathrm{dt}}=\left[\begin{array}{ll}
\frac{\partial \mathbf{X}}{\partial \mathrm{u}} & \frac{\partial \mathbf{X}}{\partial \mathrm{v}} \\
\frac{\partial \mathbf{Y}}{\partial \mathrm{u}} & \frac{\partial \mathbf{Y}}{\partial \mathrm{v}}
\end{array}\right]\left[\begin{array}{c}
\dot{\mathrm{u}} \\
\dot{\mathrm{v}}
\end{array}\right]=\left[\begin{array}{cc}
\frac{\partial \mathbf{X}(\mathrm{u}, \mathrm{v})}{\partial \mathrm{u}} & \frac{\partial \mathbf{X}(\mathrm{u}, \mathrm{v})}{\partial \mathrm{v}} \\
\frac{\partial \mathbf{Y}(\mathrm{u}, \mathrm{v})}{\partial \mathrm{u}} & \frac{\partial \mathbf{Y}(\mathrm{u}, \mathrm{v})}{\partial \mathrm{v}}
\end{array}\right]\left[\begin{array}{c}
\mathrm{v} \\
\mathrm{f}_{\mathrm{k}}
\end{array}\right]
$$

onde, $\mathrm{f}_{\mathrm{k}}=\mathrm{f}_{\mathrm{k}}\left(\mathrm{X}_{1}(\mathrm{u}, \mathrm{v}), \ldots, \mathrm{X}_{\mathrm{n}}(\mathrm{u}, \mathrm{v}), \mathrm{Y}_{1}(\mathrm{u}, \mathrm{v}), \ldots, \mathrm{Y}_{\mathrm{n}}(\mathrm{u}, \mathrm{v})\right)$.

O segundo membro da igualdade em 2.30 pode ser escrito: 


$$
\left[\begin{array}{c}
\mathrm{y}_{1} \\
\vdots \\
\mathrm{y}_{\mathrm{n}} \\
\mathrm{f}_{1}\left(\mathrm{x}_{1}, \ldots, \mathrm{x}_{\mathrm{n}}, \mathrm{y}_{1}, \ldots \mathrm{y}_{\mathrm{n}}\right) \\
\vdots \\
\mathrm{f}_{\mathrm{n}}\left(\mathrm{x}_{1}, \ldots, \mathrm{x}_{\mathrm{n}}, \mathrm{y}_{1}, \ldots \mathrm{y}_{\mathrm{n}}\right)
\end{array}\right]=\left[\begin{array}{c}
\mathrm{Y}_{1}(\mathrm{u}, \mathrm{v}) \\
\vdots \\
\mathrm{Y}_{\mathrm{n}}(\mathrm{u}, \mathrm{v}) \\
\mathrm{f}_{1}\left(\mathrm{X}_{1}(\mathrm{u}, \mathrm{v}), \ldots, \mathrm{X}_{\mathrm{n}}(\mathrm{u}, \mathrm{v}), \mathrm{Y}_{1}(\mathrm{u}, \mathrm{v}), \ldots \mathrm{Y}_{\mathrm{n}}(\mathrm{u}, \mathrm{v})\right) \\
\vdots \\
\mathrm{f}_{\mathrm{n}}\left(\mathrm{X}_{1}(\mathrm{u}, \mathrm{v}), \ldots, \mathrm{X}_{\mathrm{n}}(\mathrm{u}, \mathrm{v}), \mathrm{Y}_{1}(\mathrm{u}, \mathrm{v}), \ldots \mathrm{Y}_{\mathrm{n}}(\mathrm{u}, \mathrm{v})\right)
\end{array}\right]=\left[\begin{array}{c}
\mathbf{Y} \\
\mathbf{f}
\end{array}\right], 2.48
$$

onde,

$$
\begin{gathered}
\mathbf{Y}=\left[\begin{array}{c}
\mathrm{Y}_{1}(\mathrm{u}, \mathrm{v}) \\
\vdots \\
\mathrm{Y}_{\mathrm{n}}(\mathrm{u}, \mathrm{v})
\end{array}\right] \mathrm{e} \\
\mathbf{f}=\left[\begin{array}{c}
\mathrm{f}_{1}\left(\mathrm{X}_{1}(\mathrm{u}, \mathrm{v}), \ldots, \mathrm{X}_{\mathrm{n}}(\mathrm{u}, \mathrm{v}), \mathrm{Y}_{1}(\mathrm{u}, \mathrm{v}), \ldots \mathrm{Y}_{\mathrm{n}}(\mathrm{u}, \mathrm{v})\right) \\
\vdots \\
\mathrm{f}_{\mathrm{n}}\left(\mathrm{X}_{1}(\mathrm{u}, \mathrm{v}), \ldots, \mathrm{X}_{\mathrm{n}}(\mathrm{u}, \mathrm{v}), \mathrm{Y}_{1}(\mathrm{u}, \mathrm{v}), \ldots \mathrm{Y}_{\mathrm{n}}(\mathrm{u}, \mathrm{v})\right)
\end{array}\right]
\end{gathered}
$$

Reescreve-se 2.30 a partir de 2.47 e 2.48 obtendo-se

$$
\left[\begin{array}{cc}
\frac{\partial \mathbf{X}(\mathrm{u}, \mathrm{v})}{\partial \mathrm{u}} & \frac{\partial \mathbf{X}(\mathrm{u}, \mathrm{v})}{\partial \mathrm{v}} \\
\frac{\partial \mathbf{Y}(\mathrm{u}, \mathrm{v})}{\partial \mathrm{u}} & \frac{\partial \mathbf{Y}(\mathrm{u}, \mathrm{v})}{\partial \mathrm{v}}
\end{array}\right]\left[\begin{array}{c}
\mathrm{v} \\
\mathrm{f}_{\mathrm{k}}
\end{array}\right]=\left[\begin{array}{c}
\mathbf{Y} \\
\mathbf{f}
\end{array}\right]
$$

Nota-se em 2.51 a ausência da dependência do tempo o que faz com que o problema torne-se puramente geométrico. Escrevendo os elementos das matrizes de 2.51 tem-se, 
k-ésima linha $\longrightarrow\left[\begin{array}{cc}\frac{\partial \mathrm{X}_{1}(\mathrm{u}, \mathrm{v})}{\partial \mathrm{u}} & \frac{\partial \mathrm{X}_{1}(\mathrm{u}, \mathrm{v})}{\partial \mathrm{v}} \\ \vdots & \vdots \\ 1 & 0 \\ \vdots & \vdots \\ \frac{\partial \mathrm{X}_{\mathrm{n}}(\mathrm{u}, \mathrm{v})}{\partial \mathrm{u}} & \frac{\partial \mathrm{X}_{\mathrm{n}}(\mathrm{u}, \mathrm{v})}{\partial \mathrm{v}} \\ \frac{\partial \mathrm{Y}_{1}(\mathrm{u}, \mathrm{v})}{\partial \mathrm{u}} & \frac{\partial \mathrm{Y}_{1}(\mathrm{u}, \mathrm{v})}{\partial \mathrm{v}} \\ \vdots & \vdots \\ 0 & 1 \\ \vdots & \vdots \\ \frac{\partial \mathrm{Y}_{\mathrm{n}}(\mathrm{u}, \mathrm{v})}{\partial \mathrm{u}} & \frac{\partial \mathrm{Y}_{\mathrm{n}}(\mathrm{u}, \mathrm{v})}{\partial \mathrm{v}}\end{array}\right]\left[\begin{array}{c}\mathrm{v} \\ \mathrm{f}_{\mathrm{k}}(\mathrm{u}, \mathrm{v})\end{array}\right]=\left[\begin{array}{c}\mathrm{Y}_{1}(\mathrm{u}, \mathrm{v}) \\ \vdots \\ \mathrm{v} \\ \vdots \\ \mathrm{Y}_{\mathrm{n}}(\mathrm{u}, \mathrm{v}) \\ \mathrm{f}_{1}(\mathrm{u}, \mathrm{v}) \\ \vdots \\ \mathrm{f}_{\mathrm{k}}(\mathrm{u}, \mathrm{v}) \\ \vdots \\ \mathrm{f}_{\mathrm{n}}(\mathrm{u}, \mathrm{v})\end{array}\right]$

vale notar que,

$$
\begin{aligned}
& \frac{\partial \mathrm{X}_{\mathrm{k}}(\mathrm{u}, \mathrm{v})}{\partial \mathrm{u}}=1, \\
& \frac{\partial \mathrm{X}_{\mathrm{k}}(\mathrm{u}, \mathrm{v})}{\partial \mathrm{v}}=0, \\
& \frac{\partial \mathrm{Y}_{\mathrm{k}}(\mathrm{u}, \mathrm{v})}{\partial \mathrm{u}}=0, \mathrm{e} \\
& \frac{\partial \mathrm{Y}_{\mathrm{k}}(\mathrm{u}, \mathrm{v})}{\partial \mathrm{u}}=1 .
\end{aligned}
$$

Observando a relação 2.52, conclui-se que existem $2 n$-2 equações diferenciais não lineares independentes, uma vez que as equações correspondentes à linha $k$ são satisfeitas previamente. A solução desse sistema de equações não é um problema trivial. Porém, o sistema 2.52 deve fornecer $n$ soluções distintas e reais que devem corresponder aos $n$ modos de vibração do problema. Desta forma, escreve-se a equação do oscilador modal como: 


$$
\ddot{\mathrm{u}}^{\mathrm{r}}=\mathrm{f}_{\mathrm{k}}\left(\mathrm{X}_{1}^{\mathrm{r}}\left(\mathrm{u}^{\mathrm{r}}, \dot{\mathrm{u}}^{\mathrm{r}}\right), \ldots, \mathrm{X}_{\mathrm{n}}^{\mathrm{r}}\left(\mathrm{u}^{\mathrm{r}}, \dot{\mathrm{u}}^{\mathrm{r}}\right), \mathrm{Y}_{1}^{\mathrm{r}}\left(\mathrm{u}^{\mathrm{r}}, \dot{\mathrm{u}}^{\mathrm{r}}\right), \ldots, \mathrm{Y}_{\mathrm{n}}^{\mathrm{r}}\left(\mathrm{u}^{\mathrm{r}}, \dot{\mathrm{u}}^{\mathrm{r}}\right)\right)
$$

onde o índice $r$ corresponde ao $r$-ésimo modo de vibração.

Em alguns problemas, a descrição geométrica do modo não-linear pode coincidir com a linear, isto é, as funções que relacionam os deslocamentos e as velocidades generalizados com as variáveis modais são lineares. Estes modos de vibração são ditos similares. Porém, isto não implica dizer que a dinâmica do modo será linear, isto é, a equação do oscilador modal é não-linear. Portanto, a variedade invariante do sistema não-linear coincide integralmente com o plano que define o autoespaço do modo linearizado e, desta forma, o movimento modal se desenvolve dentro deste plano, porém a sua dinâmica é não-linear. Vale ressaltar que a escolha da variável modal é aleatória, porém deve-se tomar o cuidado de não escolher variáveis que correspondam a pontos nodais nas formas modais.

Devido à complexidade que o sistema 2.52 pode apresentar, torna-se conveniente uma solução aproximada. A aproximação que será feita adiante se utiliza de séries de potências para representar as relações modais, impondo-se respeito às equações diferenciais 2.40 que regem o sistema estrutural. Desta forma, tem-se:

$$
\begin{aligned}
& X_{i}(u, v)=a_{1 i} u+a_{2 i} v+a_{3 i} u^{2}+a_{4 i} u v+a_{5 i} v^{2}+a_{6 i} u^{3}+a_{7 i} u^{2} v+a_{8 i} u v^{2}+a_{9 i} v^{3} \\
& Y_{i}(u, v)=b_{1 i} u+b_{2 i} v+b_{3 i} u^{2}+b_{4 i} u v+b_{5 i} v^{2}+b_{6 i} u^{3}+b_{7 i} u^{2} v+b_{8 i} u v^{2}+b_{9 i} v^{3}
\end{aligned}
$$

com $i=1, \ldots, n$.

Portanto, as relações modais são aproximadas através de séries de potências de $u$ e $v$. As variáveis $a_{1 i}, \ldots, a_{9 i}$ e $b_{1 i}, \ldots, b_{9 i}$ são as incógnitas a determinar, pois são elas que definem as formas modais, isto é, as variedades invariantes.

Considera-se, também, que as funções $\mathrm{f}_{\mathrm{i}}\left(\mathrm{x}_{1}, \ldots, \mathrm{x}_{\mathrm{n}}, \mathrm{y}_{1}, \ldots, \mathrm{y}_{\mathrm{n}}\right)$, com $\mathrm{i}=1, \ldots, \mathrm{n}$ possam ser escritas como séries de potências de $x_{i}$ e $y_{i}$ na forma, 


$$
\begin{aligned}
f_{i}\left(x_{1}, \ldots, x_{n}, y_{1}, \ldots, y_{n}\right) & =B_{i j} x_{j}+C_{i j} y_{j}+ \\
& +E_{i j m} x_{j} x_{m}+F_{i j m} x_{j} y_{m}+G_{i j m} y_{j} y_{m}+ \\
& +H_{i j m p} x_{j} x_{m} x_{p}+L_{i j m p} x_{j} x_{m} y_{p}+N_{i j m p} x_{j} y_{m} y_{p}
\end{aligned}
$$

onde $i, j, m, p=1, \ldots, n$, em $B_{i j}, C_{i j}, E_{i j m}, F_{i j m}, G_{i j m}, H_{i j m p}, L_{i j m p}$ e $N_{i j m p}$.

Para determinação das relações modais substitui-se 2.58 e 2.59 em 2.51 e igualam-se os coeficientes das variáveis $\mathrm{u}, \mathrm{v}, \mathrm{u}^{2}, \mathrm{uv}, \mathrm{v}^{2}, \mathrm{u}^{3}, \mathrm{u}^{2} \mathrm{v}, \mathrm{uv}^{2}$ e $\mathrm{v}^{3}$ em ambos os membros, isto é, igualam-se os coeficientes dos termos até terceira potência. Encontra-se, portanto, um sistema de $18 n$ equações que têm por incógnitas os coeficientes $a_{i j}$ e $b_{i j}$, onde $i=1, \ldots, n$ e $j=1, \ldots, 9$. Metade dessas equações está associada à primeira equação de 2.51 :

$$
\frac{\partial \mathbf{X}(\mathrm{u}, \mathrm{v})}{\partial \mathrm{u}} \mathrm{v}+\frac{\partial \mathbf{X}(\mathrm{u}, \mathrm{v})}{\partial \mathrm{v}} \mathrm{f}_{\mathrm{k}}=\mathbf{Y}
$$

chamadas equações do primeiro grupo. Outra metade das equações está associada à segunda equação de 2.51:

$$
\frac{\partial \mathbf{Y}(\mathrm{u}, \mathrm{v})}{\partial \mathrm{u}} \mathrm{v}+\frac{\partial \mathbf{Y}(\mathrm{u}, \mathrm{v})}{\partial \mathrm{v}} \mathrm{f}_{\mathrm{k}}=\mathbf{f}
$$

chamadas equações do segundo grupo. Maiores detalhes na determinação das relações modais podem ser encontrados em Soares [86]. 


\subsection{Sistema Discretizado pelo Método dos Elementos Finitos}

Neste trabalho utiliza-se o elemento viga de eixo reto (Figura 2.1) formulado por Mazzilli [48]. Trata-se de um elemento baseado na teoria de Bernoulli-Euler que considera em sua formulação não-linearidades geométricas.
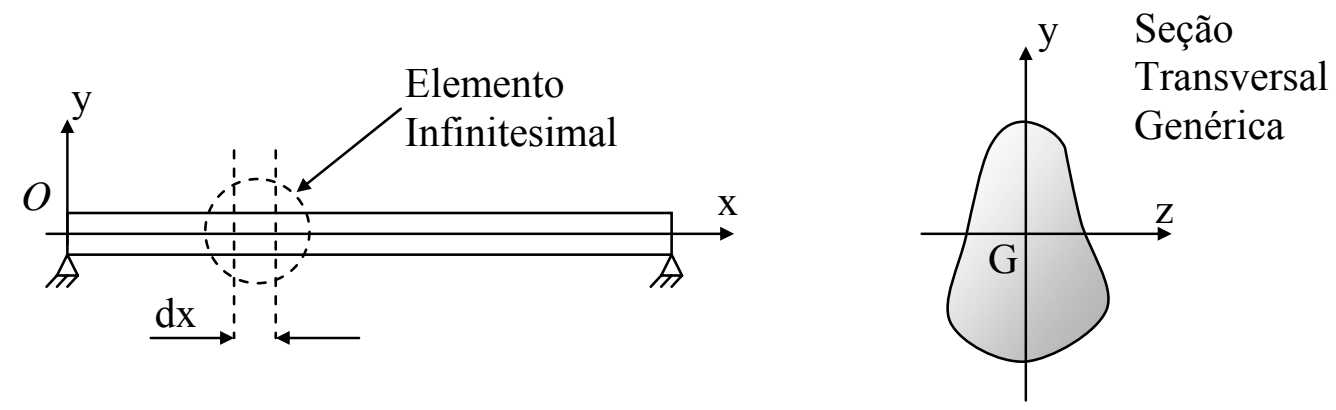

Figura 2.1: Viga de eixo reto e sua seção transversal.

A Figura 2.2 mostra um elemento plano de viga de comprimento infinitesimal primeiramente indeformado e, depois, submetido a uma deformação por flexão composta normal. Para seções genéricas, o movimento pode deixar de ser plano e a flexão normal pode ficar oblíqua. Na verdade, a formulação vale para flexão composta normal, por isso é conveniente considerar seção simétrica em relação ao eixo central de inércia Gy. A ordenada y mede a distância em relação ao eixo da viga. Os eixos $x$ e $y$ referem-se ao sistema local do elemento de viga. O ponto $O$ é origem do sistema local de coordenadas.
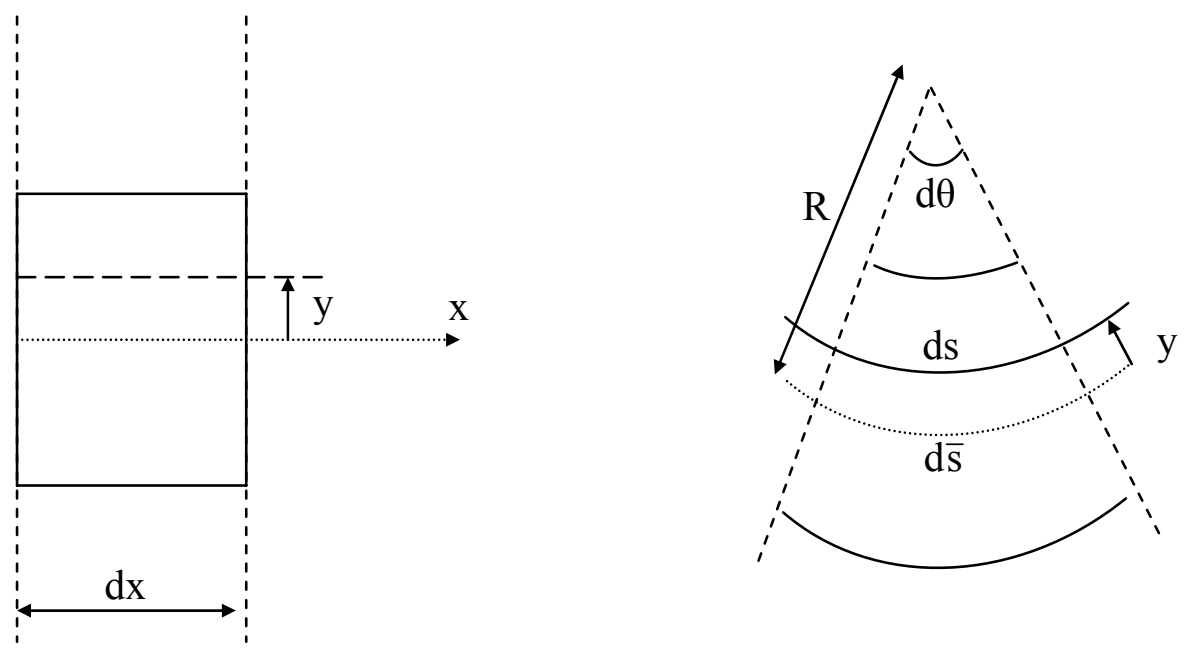

Figura 2.2: Elemento infinitesimal de viga. 
Na Figura 2.2 tem-se que:

$>$ ds é o comprimento de uma fibra sobre o eixo da viga na configuração deformada;

$>$ ds é o comprimento de uma fibra com ordenada y em relação ao eixo da viga na configuração deformada;

$>$ R é o raio de curvatura;

$>\mathrm{d} \theta$ é o ângulo entre as seções transversais.

Para proceder à formulação do elemento finito de viga não-linear, o que implica, em última análise, encontrar as suas matrizes de rigidez, de amortecimento e de massa, aplica-se a equação generalizada de Lagrange. Em um sistema com $n$ graus de liberdade tem-se:

$$
\frac{d}{d t}\left[\frac{\partial L}{\partial \dot{Q}_{r}}\right]-\left[\frac{\partial L}{\partial Q_{r}}\right]=N^{r}
$$

onde, $\mathrm{i}=1, \ldots, \mathrm{n}, \mathrm{N}^{\mathrm{r}}$ são as forças generalizadas, $\mathrm{Q}_{\mathrm{r}}$ os deslocamentos generalizados e L é o Lagrangeano do sistema,

$$
\mathrm{L}=\mathrm{T}-\mathrm{V}
$$

sendo $T$ a energia cinética e $V$ a energia potencial total.

Para vínculos holônomos, a posição do centro de massa de um elemento de massa infinitesimal dm em relação a um referencial inercial é dada pelo vetor:

$$
\mathbf{R}=\mathbf{R}\left(\mathrm{Q}_{1}, \ldots, \mathrm{Q}_{\mathrm{n}}, \mathrm{t}\right),
$$

Utilizando 2.64 a expressão da energia cinética pode ser escrita na forma, 


$$
\mathrm{T}=\frac{1}{2} \int_{\Omega}(\dot{\mathbf{R}} \cdot \dot{\mathbf{R}}) \mathrm{dm},
$$

onde $\Omega$ é a região ocupada pelo corpo indeformado e,

$$
\dot{\mathbf{R}}=\frac{\partial \mathbf{R}}{\partial \mathrm{Q}_{\mathrm{r}}} \dot{\mathrm{Q}}_{\mathrm{r}}+\frac{\partial \mathbf{R}}{\partial \mathrm{t}}
$$

Utilizando notação indicial, pode-se reescrever 2.65 como:

$$
\mathrm{T}=\frac{1}{2} \mathrm{~A}^{\mathrm{rs}} \dot{\mathrm{Q}}_{\mathrm{r}} \dot{\mathrm{Q}}_{\mathrm{s}}+\mathrm{B}^{\mathrm{r}} \dot{\mathrm{Q}}_{\mathrm{r}}+\frac{1}{2} \mathrm{C}
$$

onde,

$$
\begin{aligned}
\mathrm{A}^{\mathrm{rs}} & =\int_{\Omega}\left(\frac{\partial \mathbf{R}}{\partial \mathrm{Q}_{\mathrm{r}}} \cdot \frac{\partial \mathbf{R}}{\partial \mathrm{Q}_{\mathrm{s}}}\right) \mathrm{dm}, \\
\mathrm{B}^{\mathrm{r}} & =\int_{\Omega}\left(\frac{\partial \mathbf{R}}{\partial \mathrm{Q}_{\mathrm{r}}} \cdot \frac{\partial \mathbf{R}}{\partial \mathrm{t}}\right) \mathrm{dm}, \\
\mathrm{C} & =\int_{\Omega}\left(\frac{\partial \mathbf{R}}{\partial \mathrm{t}} \cdot \frac{\partial \mathbf{R}}{\partial \mathrm{t}}\right) \mathrm{dm} .
\end{aligned}
$$

Substituindo 2.67 em 2.62:

$$
\begin{aligned}
& \mathrm{A}^{\mathrm{rs}} \ddot{\mathrm{Q}}_{\mathrm{s}}+\left[\frac{\partial \mathrm{A}^{\mathrm{rs}}}{\partial \mathrm{Q}_{\mathrm{t}}}-\frac{1}{2} \frac{\partial \mathrm{A}^{\mathrm{st}}}{\partial \mathrm{Q}_{\mathrm{r}}}\right] \dot{\mathrm{Q}}_{\mathrm{s}} \dot{\mathrm{Q}}_{\mathrm{t}}+\frac{\partial \mathrm{A}^{\mathrm{rs}}}{\partial \mathrm{t}} \dot{\mathrm{Q}}_{\mathrm{s}}+\left[\frac{\partial \mathrm{B}^{\mathrm{r}}}{\partial \mathrm{Q}_{\mathrm{s}}}-\frac{\partial \mathrm{B}^{\mathrm{s}}}{\partial \mathrm{Q}_{\mathrm{r}}}\right] \dot{\mathrm{Q}}_{\mathrm{s}}+ \\
& +\frac{\partial \mathrm{B}^{\mathrm{r}}}{\partial \mathrm{t}}-\frac{1}{2} \frac{\partial \mathrm{C}}{\partial \mathrm{Q}_{\mathrm{r}}}=-\frac{\partial \mathrm{V}}{\partial \mathrm{Q}_{\mathrm{r}}}+\mathrm{N}^{\mathrm{r}}
\end{aligned}
$$

O elemento de viga possui 6 graus de liberdade $\overline{\mathrm{q}}_{1}, \ldots, \overline{\mathrm{q}}_{6}$ conforme Figura 2.3. Os deslocamentos $\overline{\mathrm{q}}_{1}$ e $\overline{\mathrm{q}}_{4}$ são os deslocamentos axiais correspondentes, 
respectivamente, ao nó inicial e ao nó final do elemento. Analogamente, os deslocamentos $\overline{\mathrm{q}}_{2}$ e $\overline{\mathrm{q}}_{5}$ são os deslocamentos transversais correspondentes, respectivamente, ao nó inicial e ao nó final do elemento. Os graus de liberdade $\overline{\mathrm{q}}_{3}$ e $\overline{\mathrm{q}}_{6}$ são, respectivamente, as rotações do nó inicial e do nó final do elemento.

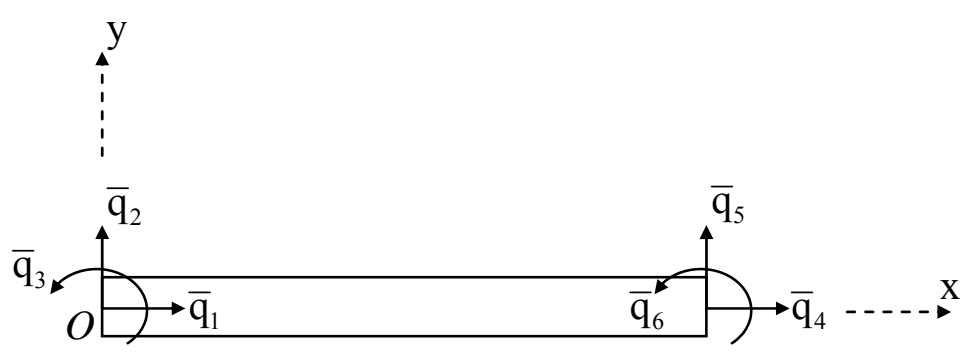

Figura 2.3: Elemento de viga com seis graus de liberdade.

Os deslocamentos horizontais e verticais do eixo da viga são dados por:

$$
\overline{\mathrm{u}}=\overline{\mathrm{q}}_{1} \phi_{1}+\ldots+\overline{\mathrm{q}}_{6} \phi_{6},
$$

$$
\overline{\mathrm{v}}=\overline{\mathrm{q}}_{1} \psi_{1}+\ldots+\overline{\mathrm{q}}_{6} \psi_{6},
$$

onde $\phi_{1}, \ldots, \phi_{6}$ e $\psi_{1}, \ldots, \psi_{6}$ são funções de forma dadas por: 


$$
\begin{array}{ll}
\psi_{1}(x)=0 & \phi_{1}(x)=1-\frac{x}{l} \\
\psi_{2}(x)=1-3 \frac{x^{2}}{l^{2}}+2 \frac{x^{3}}{l^{3}} & \phi_{2}(x)=0 \\
\psi_{3}(x)=x-2 \frac{x^{2}}{l}+\frac{x^{3}}{l^{2}} & \phi_{3}(x)=0 \\
\psi_{4}(x)=0 & \phi_{4}(x)=\frac{x}{l} \\
\psi_{5}(x)=3 \frac{x^{2}}{l^{2}}-2 \frac{x^{3}}{l^{3}} & \phi_{5}(x)=0 \\
\psi_{6}(x)=-\frac{x^{2}}{l}+\frac{x^{3}}{l^{2}} & \phi_{6}(x)=0
\end{array}
$$

A posição de qualquer ponto pertencente à viga é dada pelo vetor:

$$
\mathbf{R}=\left[\begin{array}{l}
\mathrm{x}+\mathrm{u} \\
\mathrm{y}+\mathrm{v}
\end{array}\right]
$$

Observando a Figura 2.2, a equação 2.75 pode ser escrita como,

$$
\mathbf{R}=\left[\begin{array}{c}
x+\bar{u}-y \operatorname{sen} \theta \\
y+\bar{v}+y \cos \theta-y
\end{array}\right]=\left[\begin{array}{c}
x+\bar{u}-y \operatorname{sen} \theta \\
\bar{v}+y \cos \theta
\end{array}\right]
$$

Na expressão 2.71, no lugar da energia potencial total (V) considerar-se-á a energia de deformação elástica (U), uma vez estabelecida que a parcela $\mathrm{N}^{\mathrm{r}}$ incluirá as forças conservativas. A energia de deformação é dada pela expressão:

$$
\mathrm{U}=\frac{\mathrm{E}}{2} \int_{\Omega} \varepsilon^{2} \mathrm{~d} \Omega
$$

onde, E é o módulo de elasticidade do material e $\varepsilon$ é dado por,

$$
\varepsilon=\bar{\lambda}-\mathrm{y} \theta^{\prime}-1
$$


onde, $\bar{\lambda}=\left[\left(1+\bar{u}^{\prime}\right)^{2}+\left(\bar{v}^{\prime}\right)^{2}\right]^{1 / 2}$ e $\theta^{\prime}=\frac{\mathrm{v}^{\prime \prime}\left(1+\overline{\mathrm{u}}^{\prime}\right)-\overline{\mathrm{u}}^{\prime \prime} \overline{\mathrm{v}}^{\prime}}{\left(1+\overline{\mathrm{u}}^{\prime}\right)^{2}+\left(\overline{\mathrm{v}}^{\prime}\right)^{2}}$.

Substituindo 2.76 e 2.77 em 2.71, encontra-se a equação de movimento da viga no sistema local, que é dada por

$$
\bar{m}_{r s} \ddot{\bar{q}}_{s}+\bar{d}_{r s} \dot{\bar{q}}_{s}+\bar{U}_{r}=\bar{f}_{i}
$$

onde, $\overline{\mathrm{m}}_{\mathrm{rs}}, \overline{\mathrm{d}}_{\mathrm{rs}}$ e $\overline{\mathrm{U}}_{\mathrm{r}}, \mathrm{r}, \mathrm{s}=1, \ldots, 6$ são, respectivamente, os termos da matriz de massa, da matriz de amortecimento e do vetor de forças elásticas do elemento. Ainda na equação $2.79, \bar{f}_{i}$ representa um termo do vetor das forças nodais do elemento no sistema local. Vale notar que, para determinar os modos não-lineares, a equação global de movimento deve ser igualada a zero, sendo, portanto, homogênea.

Em sistemas não-lineares, a equação de movimento tem a seguinte forma

$$
\overline{\mathbf{m}}(\overline{\mathbf{q}}) \ddot{\overline{\mathbf{q}}}+\overline{\mathbf{d}}(\overline{\mathbf{q}}, \dot{\overline{\mathbf{q}}}) \dot{\overline{\mathbf{q}}}+\overline{\mathbf{k}}(\overline{\mathbf{q}}) \overline{\mathbf{q}}=\overline{\mathbf{f}}
$$

Detalhes sobre a determinação de 2.80 podem ser encontrados em [48]. Reescrevendo 2.80 e utilizando notação indicial, vem

$$
\bar{m}_{i j} \ddot{\bar{q}}_{j}+\bar{d}_{i j} \dot{\bar{q}}_{j}+\bar{k}_{i j} \bar{q}_{j}=\bar{f}_{i},
$$

onde

$$
\begin{gathered}
\overline{\mathrm{m}}_{\mathrm{ij}}=\overline{\mathrm{M}}_{\mathrm{ij}}^{0}+\overline{\mathrm{M}}_{\mathrm{ijk}}^{1} \overline{\mathrm{q}}_{\mathrm{k}}+\overline{\mathrm{M}}_{\mathrm{ijk} l}^{2} \overline{\mathrm{q}}_{\mathrm{k}} \overline{\mathrm{q}}_{l}, \\
\overline{\mathrm{d}}_{\mathrm{ij}}=\overline{\mathrm{D}}_{\mathrm{ij}}^{0}+\overline{\mathrm{D}}_{\mathrm{ijk}}^{1} \dot{\overline{\mathrm{q}}}_{\mathrm{k}}+\overline{\mathrm{D}}_{\mathrm{ijk} l}^{1} \dot{\overline{\mathrm{q}}}_{\mathrm{k}} \overline{\mathrm{q}}_{l}, \\
\overline{\mathrm{k}}_{\mathrm{ij}}=\overline{\mathrm{K}}_{\mathrm{ij}}^{0}+\overline{\mathrm{K}}_{\mathrm{ijk}}^{1} \overline{\mathrm{q}}_{\mathrm{k}}+\overline{\mathrm{K}}_{\mathrm{ijk} l}^{2} \overline{\mathrm{q}}_{\mathrm{k}} \overline{\mathrm{q}}_{l} \mathrm{e}
\end{gathered}
$$




$$
\overline{\mathrm{k}}_{\mathrm{ij}} \overline{\mathrm{q}}_{\mathrm{j}}=\overline{\mathrm{U}}_{\mathrm{i}} \cdot
$$

A matriz de rotação que relaciona coodenadas locais com as coordenadas globais pode ser escrita na forma (ver [77]):

$$
\mathbf{a}=\left[\begin{array}{cccccc}
\cos \theta & \operatorname{sen} \theta & 0 & 0 & 0 & 0 \\
-\operatorname{sen} \theta & \cos \theta & 0 & 0 & 0 & 0 \\
0 & 0 & 1 & 0 & 0 & 0 \\
0 & 0 & 0 & \cos \theta & \operatorname{sen} \theta & 0 \\
0 & 0 & 0 & -\operatorname{sen} \theta & \cos \theta & 0 \\
0 & 0 & 0 & 0 & 0 & 1
\end{array}\right]
$$

Aplicando a matriz de rotação a (ver [77]) em 2.81 e realizando as transformações internas às matrizes, tem-se a seguinte equação de movimento do elemento de viga no sistema global:

$$
m_{i j} \ddot{q}_{j}+d_{i j} \dot{q}_{j}+k_{i j} q_{j}=f_{i},
$$

onde

$$
\begin{gathered}
\mathrm{m}_{\mathrm{ij}}=\mathrm{M}_{\mathrm{ij}}^{0}+\mathrm{M}_{\mathrm{ijk}}^{1} \mathrm{q}_{\mathrm{k}}+\mathrm{M}_{\mathrm{ijk} l}^{2} \mathrm{q}_{\mathrm{k}} \mathrm{q}_{l}, \\
\mathrm{~d}_{\mathrm{ij}}=\mathrm{D}_{\mathrm{ij}}^{0}+\mathrm{D}_{\mathrm{ijk}}^{1} \dot{\mathrm{q}}_{\mathrm{k}}+\mathrm{D}_{\mathrm{ijk} l}^{1} \dot{\mathrm{q}}_{\mathrm{k}} \mathrm{q}_{l}, \\
\mathrm{k}_{\mathrm{ij}}=\mathrm{K}_{\mathrm{ij}}^{0}+\mathrm{K}_{\mathrm{ijk}}^{1} \mathrm{q}_{\mathrm{k}}+\mathrm{K}_{\mathrm{ijk} l}^{2} \mathrm{q}_{\mathrm{k}} \mathrm{q}_{l},
\end{gathered}
$$

sendo

$$
\begin{gathered}
\mathrm{M}_{l \mathrm{k}}^{0}=\mathrm{a}_{l \mathrm{i}}^{\mathrm{t}} \overline{\mathrm{M}}_{\mathrm{ij}}^{0} \mathrm{a}_{\mathrm{jk}}, \\
\mathrm{M}_{\mathrm{n} \mathrm{m} l}^{1}=\mathrm{a}_{\mathrm{ni}}^{\mathrm{t}} \overline{\mathrm{M}}_{\mathrm{ijk}}^{1} \mathrm{a}_{\mathrm{k} l} \mathrm{a}_{\mathrm{jm}},
\end{gathered}
$$


$\mathrm{M}_{\mathrm{pomn}}^{2}=\mathrm{a}_{\mathrm{ni}}^{\mathrm{t}} \overline{\mathrm{M}}_{\mathrm{ijkl}}^{2} \mathrm{a}_{\mathrm{km}} \mathrm{a}_{l \mathrm{n}} \mathrm{a}_{\mathrm{jo}}$

$\mathrm{D}_{l \mathrm{k}}^{0}=\mathrm{a}_{l \mathrm{i}}^{\mathrm{t}} \overline{\mathrm{D}}_{\mathrm{ij}}^{0} \mathrm{a}_{\mathrm{jk}}$

$$
\mathrm{D}_{\mathrm{n} \mathrm{m} l}^{1}=\mathrm{a}_{\mathrm{ni}}^{\mathrm{t}} \overline{\mathrm{D}}_{\mathrm{ijk}}^{1} \mathrm{a}_{\mathrm{k} l} \mathrm{a}_{\mathrm{jm}}
$$

$\mathrm{D}_{\mathrm{pomn}}^{2}=\mathrm{a}_{\mathrm{n} i}^{\mathrm{t}} \overline{\mathrm{D}}_{\mathrm{ijkl} \mathrm{a}_{\mathrm{km}}}^{2} \mathrm{a}_{l \mathrm{n}} \mathrm{a}_{\mathrm{jo}}$

$$
\mathrm{K}_{l \mathrm{k}}^{0}=\mathrm{a}_{l \mathrm{i}}^{\mathrm{t}} \overline{\mathrm{K}}_{\mathrm{ij}}^{0} \mathrm{a}_{\mathrm{jk}}
$$

$$
\mathrm{K}_{\mathrm{nm} l}^{1}=\mathrm{a}_{\mathrm{ni}}^{\mathrm{t}} \overline{\mathrm{K}}_{\mathrm{ijk}}^{1} \mathrm{a}_{\mathrm{k} l} \mathrm{a}_{\mathrm{jm}}
$$

$$
\mathrm{K}_{\mathrm{pomn}}^{2}=\mathrm{a}_{\mathrm{ni}}^{\mathrm{t}} \overline{\mathrm{K}}_{\mathrm{ijk} l}^{2} \mathrm{a}_{\mathrm{km}} \mathrm{a}_{l \mathrm{n}} \mathrm{a}_{\mathrm{jo}} .
$$

Uma vez encontradas as equações de movimento dos elementos no sistema global segue-se a montagem da equação de movimento global. O sistema de equações encontrado é de segunda ordem. Para aplicar-se o procedimento proposto por Shaw e Pierre, é necessário transformar este sistema de $n$ equações diferenciais de segunda ordem em um sistema de $2 n$ equações diferenciais de primeira ordem.

Alguns sistemas estruturais podem apresentar uma configuração deformada inicial a partir da qual se deseja determinar os modos não-lineares. Esse é o caso dos exemplos tratados neste estudo: risers retos pré-tensionados e risers em catenária. Para tanto, admite-se que o vetor de deslocamentos generalizados possa ser escrito na forma:

$$
\mathbf{q}=\mathbf{q}_{C}+\mathbf{q}_{0},
$$

onde, $\mathbf{q}_{c}$ é o vetor de deslocamentos generalizados a partir da configuração deformada e $\mathbf{q}_{0}$ é o vetor de deslocamentos generalizados para a determinação da configuração deformada inicial. 
Substituindo 2.100 em 2.87, 2.88, 2.89 e 2.90 vem:

$$
m_{i j} \ddot{q}_{c_{j}}+d_{i j} \dot{q}_{c_{j}}+k_{i j}\left(q_{c_{j}}+q_{0_{j}}\right)=f_{i},
$$

onde

$$
\begin{gathered}
m_{i j}=M_{i j}^{0}+M_{i j k}^{1}\left(q_{c_{k}}+q_{0_{k}}\right)+M_{i j \mathrm{k} l}^{2}\left(q_{c_{k}}+q_{0_{k}}\right)\left(q_{c_{l}}+q_{0_{l}}\right), \\
d_{i j}=D_{i j}^{0}+D_{i j k}^{1}\left(\dot{q}_{c_{k}}+\dot{q}_{0_{k}}\right)+D_{i j k l}^{1}\left(\dot{q}_{c_{k}}+\dot{q}_{0_{k}}\right)\left(q_{c_{l}}+q_{0_{l}}\right), \\
k_{i j}=K_{i j}^{0}+K_{i j k}^{1}\left(q_{c_{k}}+q_{0_{k}}\right)+K_{i j \mathrm{j} l}^{2}\left(q_{c_{k}}+q_{0_{k}}\right)\left(q_{c_{l}}+q_{0_{l}}\right) .
\end{gathered}
$$

A equação 2.101 pode ser escrita na forma:

$$
m_{c_{i j}} \ddot{q}_{c_{j}}+d_{c_{i j}} \dot{q}_{c_{j}}+k_{c_{i j}} q_{c_{j}}=f_{i},
$$

com

$$
\begin{aligned}
& m_{C_{i j}}=\left(M_{i j}^{0}+M_{i j k}^{1 Q I}+M_{i \mathrm{jk} l}^{2 Q Q I}\right)+ \\
& +\left(M_{i j k}^{1}+M_{i \mathrm{jk} l}^{2 Q I}+M_{i \mathrm{jk} l}^{2 Q I I}\right) q_{c_{k}}+, \\
& +M_{i \mathrm{jk} l}^{2} q_{c_{k}} q_{c_{l}} \\
& d_{c_{i j}}=D_{i j}^{0}+\left(D_{i j k}^{1}+D_{i j k l}^{2} q_{0_{l}}\right) \dot{q}_{c_{k}}+D_{i j k l}^{2} \dot{q}_{c_{k}}, \\
& k_{i j}=\left(K_{i j}^{0}+K_{i j}^{1 Q}+K_{i j}^{2 Q Q I}+K_{i j}^{2 Q Q I I}+K_{i j}^{2 Q Q I I I}\right)+ \\
& +\left(K_{i j k}^{1}+K_{i j k}^{2 Q I}+K_{i j k}^{2 Q I I}+K_{i j k}^{2 Q I I I}\right) q_{c_{k}}+\text {, } \\
& +K_{i \mathrm{jk} l}^{2} q_{C_{k}} q_{C_{l}}
\end{aligned}
$$


onde

$$
\begin{aligned}
& M_{i j k}^{1} q_{0_{k}}=M_{i j}^{1 Q I} \\
& M_{i \mathrm{jk} l}^{2} q_{0_{k}} q_{0_{l}}=M_{i j}^{2 Q Q I} \\
& M_{i \mathrm{jk} l}^{2} q_{0_{k}}=M_{i j l}^{2 Q I} \\
& M_{i \mathrm{jk} l}^{2} q_{0_{l}}^{2}=M_{i j k}^{2 Q I I}
\end{aligned}
$$

e

$$
\begin{aligned}
& K_{i k}^{1 Q}=K_{i j k}^{1} q_{0} \\
& K_{i k}^{2 Q Q I}=K_{i j k l}^{2} q_{0_{l}} q_{0_{j}} \\
& K_{i l}^{2 Q Q I I}=K_{i j k l}^{2} q_{0_{k}} q_{0_{j}} \\
& K_{i j}^{2 Q Q I I I}=K_{i j k l}^{2} q_{0_{k}} q_{0_{l}} . \\
& K_{i k l}^{2 Q I}=K_{i j k l}^{2} q_{0_{j}} \\
& K_{i j k}^{2 Q I I}=K_{i j k l}^{2} q_{0_{l}} \\
& K_{i j l}^{2 Q I I I}=K_{i j k l}^{2} q_{0_{k}}
\end{aligned}
$$

As equações 2.100 a 2.110 apresentam as atualizações feitas no software modonl para gerar o software modonlr. 


\subsection{Multi-modos}

Segue-se a linha proposta por Shaw et al [11], [12], [13] e [79], em que os multi-modos são entendidos como uma extensão dos modos não-lineares singulares de vibração no caso em que há interação entre $n \geq 2$ desses modos. Tais interações podem ser fruto de situações de ressonância interna. Os movimentos oriundos se desenvolvem em uma variedade invariante no espaço de fase do sistema cuja dimensão é igual a duas vezes o número de modos que interagem entre si. Os multimodos são, localmente, expressos por combinações lineares dos modos lineares.

O desenvolvimento das expressões relacionadas aos multi-modos envolve grande trabalho algébrico e extensa manipulação de dados. Portanto, a opção no decorrer do texto será a de explicar a metodologia fazendo referência a resultados encontrados na literatura e, tanto quanto possível, omitindo a apresentação de extensas expressões intermediárias que não sejam absolutamente necessárias ao entendimento da técnica.

Considerem-se as equações de movimento de estruturas discretizadas pelo método dos elementos finitos:

$$
M_{r s} \ddot{p}_{s}+D_{r s} \dot{p}_{s}+U_{, r}=F_{r}
$$

com

$$
\begin{gathered}
M_{r s}={ }^{0} M_{r s}+{ }^{1} M_{r s}^{i} p_{i}+{ }^{2} M_{r s}^{i j} p_{i} p_{j}, \\
D_{r s}={ }^{0} D_{r s}+{ }^{1} D_{r s}^{i} \dot{p}_{i}+{ }^{2} D_{r s}^{i j} \dot{p}_{i} p_{j}, \\
U_{, r}={ }^{0} K_{r s} p_{s}+{ }^{1} K_{r s}^{i} p_{i} p_{s}+{ }^{2} K_{r s}^{i j} p_{i} p_{j} p_{s},
\end{gathered}
$$

onde M, D são, respectivamente, as matrizes de massa e de amortecimento viscoso e $\mathbf{U}$ e $\mathbf{F}$ os vetores de forças restauradoras elásticas e de carregamentos. Estudam-se apenas vetores de carregamentos nulos ou, quando se tratar de vibrações em torno da 
configuração deformada de equilíbrio, vetores de carregamentos estáticos, excetuando os exemplos onde seja dito explicitamente o contrário, como nos exemplos que consideram vibrações induzidas por vórtices. Os índices $r, s, i, j$ assumem valores de 1 a n, onde n é o número de graus de liberdade da estrutura.

Segundo o procedimento usual do método das múltiplas escalas [60], escrevem-se as coordenadas generalizadas em função do parâmetro adimensional $\varepsilon$ $(0<\varepsilon<<1)$

$$
p_{s}(t)=p_{s 0}+\varepsilon p_{s 1}+\varepsilon^{2} p_{s 2}+\varepsilon^{3} p_{s 3}+\ldots
$$

onde, $\quad p_{s 1}=p_{s 1}\left(T_{0}, T_{1}, T_{2}, \ldots\right), \quad p_{s 2}=p_{s 2}\left(T_{0}, T_{1}, T_{2}, \ldots\right)$ e $p_{s 3}=p_{s 3}\left(T_{0}, T_{1}, T_{2}, \ldots\right) ; \quad p_{s 0}$ caracteriza a configuração estática de equilíbrio; $s=1,2,3 \ldots$

Mostra-se que:

$$
\begin{gathered}
\frac{d}{d t}=D_{0}+\varepsilon D_{1}+\varepsilon^{2} D_{2}+\ldots \\
\frac{d^{2}}{d t^{2}}=D_{0}^{2}+2 \varepsilon D_{0} D_{1}+\varepsilon^{2}\left(2 D_{0} D_{2}+D_{1}^{2}\right)+\ldots
\end{gathered}
$$

onde $T_{i}=\varepsilon^{i} t$ e $D_{i}^{n}=\frac{d^{n}}{d T_{i}^{n}}$.

Substitui-se a equação 2.113 nas equações de movimento 2.111. Após a substituição, encontra-se a expressão com todos os termos em $\varepsilon, \varepsilon^{2}$ e $\varepsilon^{3}$. Coletando os termos em $\varepsilon$ encontra-se:

\section{Equações de ordem $\varepsilon$}




$$
{ }^{0} M_{r s} D_{0}^{2} p_{s 1}+{ }^{0} D_{r s} D_{0} p_{s 1}+{ }^{0} K_{r s} p_{s 1}=0
$$

Os autovalores são da seguinte forma:

$$
\Psi_{u}=\alpha_{u}+i \beta_{u}
$$

onde, $u$ indica o modo.

Caracterizando o primeiro modo ressonante pelo índice I e o segundo pelo índice II, a solução da equação 2.115 pode ser escrita na forma:

$$
p_{S 1}=A_{S 1}^{I} e^{\Psi_{I} T_{0}}+A_{S 1}^{I I} e^{\Psi_{I I} T_{0}}+\text { c.C. }
$$

onde $A_{s 1}^{I}=A_{s 1}^{I}\left(T_{1}, T_{2}, \ldots\right)$ e $A_{s 1}^{I I}=A_{s 1}^{I I}\left(T_{1}, T_{2}, \ldots\right)$.

Convém notar que é justamente esse o ponto em que se deve preparar as expressões a fim de que, nas ordens superiores, admitam a possibilidade de acoplamento modal, o que se consegue caracterizando a solução de ordem $\varepsilon$ não mais por apenas um modo, senão pelos dois modos acoplados (internamente ressonantes).

É nessa etapa que se garante que o multi-modo é tangente aos autoespaços do sistema linearizado. É interessante ressaltar que é essa seleção que confere ao multimodo seu caráter invariante, visto que confina o movimento (na ordem $\varepsilon$ ) aos modos lineares selecionados e, como se perceberá nas ordens $\varepsilon^{2}$ e $\varepsilon^{3}$, às combinações nãolineares desses.

Equações de ordem $\varepsilon^{2}$

Coletando os termos em $\varepsilon^{2}$ vem: 


$$
\begin{aligned}
& { }^{0} M_{r s}^{*} D_{0}^{2} p_{s 2}+{ }^{0} D_{r s}^{*} D_{0} p_{s 2}+{ }^{0} K_{r s}^{*} p_{s 2}= \\
& -2\left({ }^{0} M_{r s}+{ }^{1} M_{r s}^{i} p_{i 0}+{ }^{2} M_{r s}^{i j} p_{i 0} p_{j 0}\right) D_{0} D_{1} p_{s 1} \\
& -{ }^{0} D_{r s} D_{1} p_{s 1}- \\
& \left({ }^{1} M_{r s}^{i} p_{i 1}+{ }^{2} M_{r s}^{i j} p_{j 0} p_{i 1}+{ }^{2} M_{r s}^{j i} p_{j 0} p_{i 1}\right){ }^{1} M_{r s}^{i} p_{i 1} D_{0}^{2} p_{s 1}- \\
& \left({ }^{1} D_{r s}^{i}+{ }^{2} D_{r s}^{i j} p_{j 0}\right)\left(D_{0} p_{i 1} D_{0} p_{s 1}\right)- \\
& \left({ }^{1} K_{r s}^{i} p_{i 1}+{ }^{2} K_{r s}^{i j} p_{j 0} p_{i 1}+{ }^{2} K_{r s}^{i j} p_{j 0} p_{i 1}+{ }^{2} K_{r j}^{s i} p_{j 0} p_{i 1}\right) p_{s 1}
\end{aligned}
$$

onde ${ }^{0} M_{r s}^{*},{ }^{0} D_{r s}^{*}$ e ${ }^{0} K_{r s}^{*}$ são os elementos das matrizes de massa, amortecimento e rigidez escritas para a configuração deformada de equilíbrio (provenientes de teoria de segunda ordem).

A solução de 2.118 pode ser escrita na forma:

$$
\begin{aligned}
& p_{\mathrm{s} 2}=A_{\mathrm{S} 2}^{I} e^{\Psi_{I} T_{0}}+A_{\mathrm{S} 2}^{I I} e^{\Psi_{I I} T_{0}}+B_{\mathrm{S} 2}^{I I} e^{2 \Psi_{I I} T_{0}}+C_{\mathrm{S} 2} e^{\left(\Psi_{I}+\Psi_{I I}\right) T_{0}}+ \\
& F_{S 2}^{I} e^{2 \alpha_{I} T_{0}}+F_{S 2}^{I I} e^{2 \alpha_{I I} T_{0}}+\text { c.c. }
\end{aligned}
$$

A determinação de $A_{s 2}^{I}, A_{s 2}^{I I}, B_{s 2}^{I I}, C_{s 2}, F_{s 2}^{I}$ e $F_{s 2}^{I I}$ pode ser encontrada em [6].

\section{Equações de ordem $\varepsilon^{3}$}

Coletando-se os termos $\varepsilon^{3}$ vem: 


$$
\begin{aligned}
& { }^{0} M_{r s}^{*} D_{0}^{2} p_{s 3}+{ }^{0} D_{r s}^{*} D_{0} p_{s 3}+{ }^{0} K_{r s}^{*} p_{s 3}= \\
& -2{ }^{0} M_{r s}^{i j} D_{0} D_{2} p_{s 1}-{ }^{0} D_{r s}^{*} D_{2} p_{s 1}-2{ }^{1} M_{r s}^{i} p_{i 0} D_{0} D_{2} p_{s 1}- \\
& 2{ }^{2} M_{r s}^{i j} p_{i 0} p_{j 0} D_{0} D_{2} p_{s 1}-\left({ }^{2} M_{r s}^{i j}+{ }^{2} M_{r s}^{j i}\right) p_{j 0} p_{i 1} D_{0}^{2} p_{s 2}- \\
& \left({ }^{2} M_{r s}^{i j}+{ }^{2} M_{r s}^{j i}\right) p_{j 0} p_{i 2} D_{0}^{2} p_{s 1}-\left({ }^{2} D_{r s}^{i j}+{ }^{2} D_{r i}^{s j}\right) p_{j 0} D_{0} p_{i 1} D_{0} p_{s 2}- \\
& \left({ }^{2} K_{r s}^{i j}+{ }^{2} K_{r s}^{j i}+{ }^{2} K_{r i}^{j s}+{ }^{2} K_{r i}^{s j}+{ }^{2} K_{r j}^{i s}+{ }^{2} K_{r j}^{s i}\right) p_{j 0} p_{i 1} p_{s 2}- \\
& { }^{2} M_{r s}^{i j} p_{i 1} p_{j 1} D_{0}^{2} p_{s 1}-{ }^{2} D_{r s}^{i j} p_{j 1} D_{0} p_{i 1} D_{0} p_{s 1}-{ }^{2} K_{r s}^{i j} p_{i 1} p_{j 1} p_{s 1}- \\
& { }^{1} M_{r s}^{i} p_{i 1} D_{0}^{2} p_{s 2}-{ }^{1} M_{r s}^{i} p_{i 2} D_{0}^{2} p_{s 1}-\left({ }^{1} D_{r s}^{i}+{ }^{1} D_{r i}^{s}\right) D_{0} p_{i 1} D_{0} p_{s 2}- \\
& \left({ }^{1} K_{r s}^{i}+{ }^{1} K_{r i}^{s}\right) p_{i 1} p_{s 2}
\end{aligned}
$$

A solução de 2.120 pode ser escrita na forma:

$$
\begin{aligned}
& p_{s 3}=A_{S 3}^{I} e^{\Psi_{I} T_{0}}+A_{S 3}^{I I} e^{\Psi_{I I} T_{0}}+H_{S 3}^{I} e^{\left(2 \Psi_{I}+\Psi_{I I}\right) T_{0}}+H_{S 3}^{I I} e^{\left(\Psi_{I}+2 \Psi_{I I}\right) T_{0}}+ \\
& I_{S 3} e^{\left(\bar{\Psi}_{I}+2 \Psi_{I I}\right) T_{0}}+J_{S 3} e^{3 \Psi_{I I} T_{0}}+\text { c.c. }
\end{aligned}
$$

A determinação de $A_{s 3}^{I}, A_{s 3}^{I I}, H_{s 3}^{I}, H_{s 3}^{I I}, I_{s 3}$ e $J_{s 3}$ pode ser encontrada em [6]. A teoria e os modelos matemáticos apresentados nesta seção são as bases do programa para cálculo dos multi-modos não-lineares elaborado por Baracho Neto [6]. 


\section{Capítulo 3}

\section{Risers Retos Sujeitos a Carregamentos Axiais}

Este capítulo tem o objetivo de estudar os modos não-lineares de risers retos submetidos a diferentes condições de carregamento axial. Evidencia-se a importância de tais estruturas na exploração em águas ultra profundas em arranjos conhecidos como Riser Towers. Em geral, estes arranjos se demonstram soluções, além de tecnicamente desafiadoras, mais econômicas, se comparadas aos risers em catenária, para profundidades iguais ou superiores a $1800 \mathrm{~m}$.

Nas seções seguintes, serão abordados três casos de riser reto: o primeiro caso apresenta força normal constante de tração ao longo de todo o comprimento, ou seja, o peso próprio é desconsiderado; o exemplo seguinte trata do mesmo riser, porém com o acréscimo do peso próprio que leva à variação da força normal em função da profundidade; e no último caso admite-se também, além da tensão de tração e do peso próprio, a existência de corrente de velocidade constante ao longo do riser e a variação da protensão devido ao peso próprio.

O primeiro caso será estudado por duas abordagens. A primeira delas se trata de uma abordagem analítica. A segunda é feita utilizando-se o método dos elementos finitos. As duas abordagens apresentam boa concordância e resultados adequados para amplitudes reduzidas. Com o aumento das amplitudes, os dois métodos apresentam respostas distintas. O segundo exemplo também será abordado por ambas as metodologias, mas no terceiro caso será utilizado apenas o M.E.F., pois este método é mais adequado para sistemas complexos com relação a carregamentos, geometria, condições de contorno, etc. 


\subsection{Sem Peso Próprio}

Nesta seção, estuda-se um riser reto com extremidades articuladas, porém fixas após aplicação da protensão inicial $N_{0}$. Ressalta-se que se trata de uma simplificação do problema real, onde a embarcação impõe o deslocamento na superfície. O peso próprio é desconsiderado. A figura 3.1 apresenta o sistema estrutural de forma esquemática.
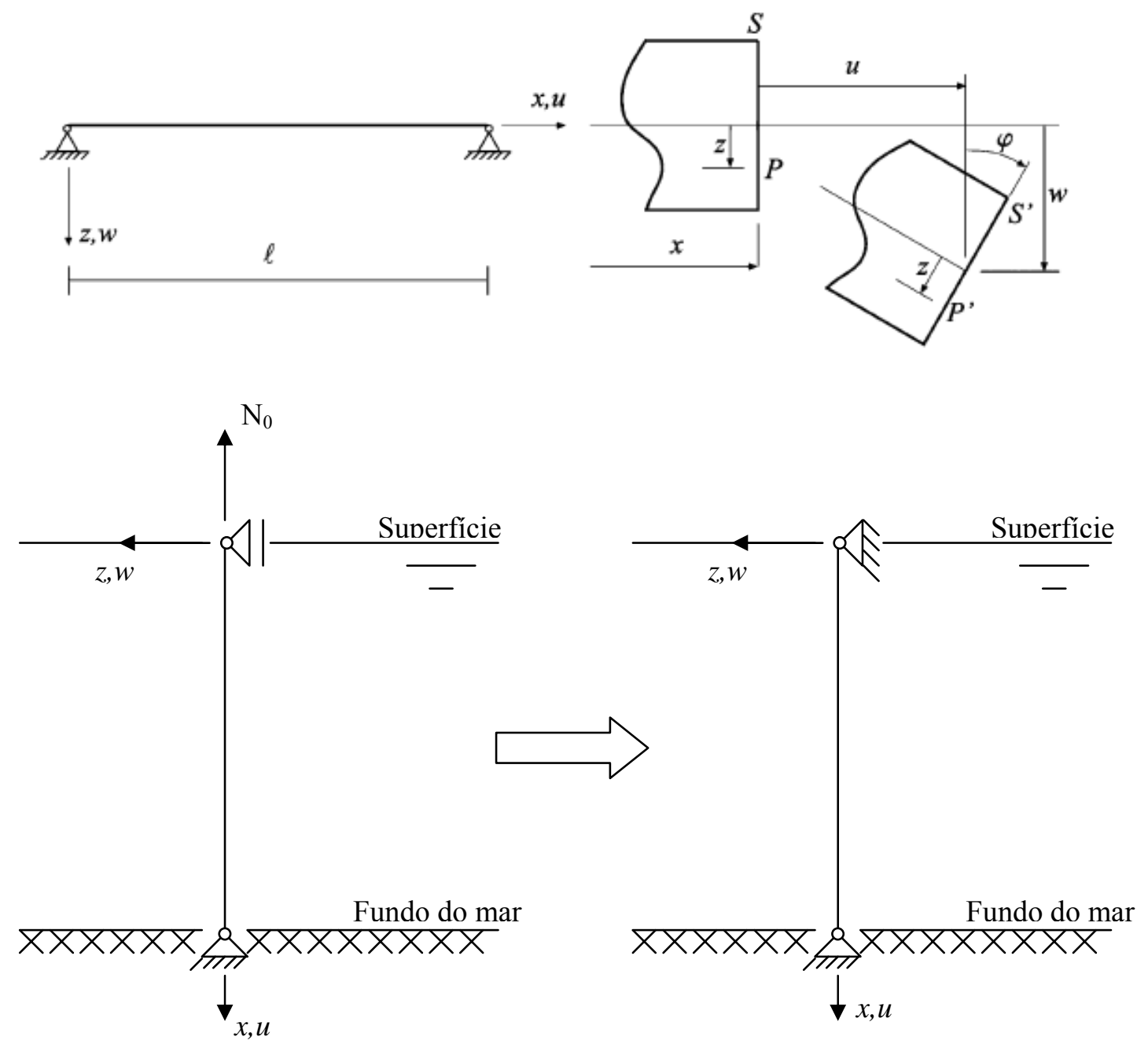

Figura 3.1- Riser reto esquemático carregado axialmente com força normal de tração constante $N_{0}$; Modelo de Bernoulli-Euler. 
Na figura 3.1, $x$ é eixo longitudinal do riser e $u$ é o deslocamento de um ponto $\mathrm{P}$ qualquer, pertencente ao eixo, nesta direção. O eixo perpendicular ao eixo $x$ é o eixo $z$ e $w$ é o deslocamento na direção de $z$ do ponto P. Denomina-se $\mathrm{S}$ uma seção transversal qualquer e $\varphi$ a rotação desta seção. P' e S' são as posições finais do ponto $\mathrm{P}$ e da seção transversal $\mathrm{S}$, respectivamente. $\mathrm{O}$ comprimento do riser é indicado por $\ell$. Assumindo a hipótese cinemática de Bernoulli-Euler (ver Mazzilli et al [51]) tem-se:

$$
\begin{aligned}
& u_{P}=u-z \sin \varphi \cong u-z w^{\prime} \\
& w_{P}=w+z(\cos \varphi-1) \cong w, \\
& \varphi=\arctan \left(\frac{w^{\prime}}{1+u^{\prime}}\right) \cong w^{\prime}
\end{aligned}
$$

onde o índice $P$ é relativo ao ponto $\mathrm{P}$ genérico.

A deformação, em um ponto $\mathrm{P}$ genérico do riser, é dada por:

$$
\varepsilon_{P}=u_{P}^{\prime}+\frac{1}{2}\left(u_{P}^{\prime}\right)^{2}+\frac{1}{2}\left(w_{P}^{\prime}\right)^{2} \cong u^{\prime}-z w^{\prime \prime}+\frac{1}{2}\left(w^{\prime}\right)^{2}=\varepsilon-z w^{\prime \prime}
$$

onde $\varepsilon$ é a deformação axial,

$$
\varepsilon=u^{\prime}+\frac{1}{2}\left(w^{\prime}\right)^{2}
$$

A equação de movimento transversal é derivada do princípio de Hamilton, ver [68]:

$$
m \ddot{w}+E I w^{I V}-E A\left[\frac{u_{\ell}-u_{0}}{\ell}+\frac{1}{2 \ell} \int_{0}^{\ell} w^{\prime 2} d x\right] w^{\prime \prime}=0,
$$

onde $E A$ e $E I$ são a rigidez axial e a rigidez à flexão; $m$ é a massa por unidade de comprimento. $\mathrm{O}$ deslocamento axial $u_{0}$ é o deslocamento axial imposto em $x=0$, ou seja, aplica-se uma força $N_{0}$ e permite-se que o vínculo tenha um deslocamento $u_{0}$ na 
direção $x$. Em seguida, restringe-se o vínculo, impedindo deslocamentos na direção $\mathrm{x}$ durante a dinâmica do sistema. Portanto, o riser apresenta uma configuração deformada estática inicial dada pela introdução de $u_{0}$ e, conseqüentemente, de $N_{0}$. Neste estudo $u_{\ell}=u(\ell, t)=0$.

A partir da equação 3.4 e com o auxílio das equações $3.1,3.2$ e 3.3 encontrase:

$$
m \ddot{w}+E I w^{i v}-N(w, t) w^{\prime \prime}=0,
$$

onde $N(w, t)$ é a força normal ou axial, e pode ser escrita como:

$$
N(w, t)=N_{0}+\frac{E A}{2 l} \int_{0}^{l} w^{\prime} d x
$$

sendo, $N_{0}$ a força normal constante ao longo do comprimento do riser originada pela introdução do deslocamento $u_{0}$. Por ora, o amortecimento será desconsiderado, sendo introduzido nos exemplos que estudam as vibrações induzidas por vórtices.

Aplicando o método das múltiplas escalas para a solução da equação 3.5 encontra-se:

$$
\omega_{k}=\frac{k \pi}{\ell} \sqrt{\frac{N_{0}}{m}+\frac{\alpha k^{2} \pi^{2}}{\ell^{2}}},
$$

onde $\omega_{k}$ é a freqüência do modo linear $k$ e $\alpha$ vale:

$$
\alpha=\frac{E I}{m} .
$$

A equação não-linear do oscilador modal é dada por (ver [51]) 


$$
\ddot{U}_{k}+\omega_{k}^{2} U_{k}+\Lambda_{k} U_{k}^{3}=0
$$

onde $U_{k}$ é o deslocamento modal e $\Lambda_{k}$ vale:

$$
\Lambda_{k}=\left(\frac{E A}{4 m}\right) \frac{k^{4} \pi^{4}}{\ell^{4}}
$$

A equação 3.9 é obtida aplicando o método das múltiplas escalas em 3.5. Admite-se que a solução de 3.5 é da forma:

$$
w(x, t)=\varepsilon w_{1}\left(x, T_{0}, T_{1} \ldots\right)+\varepsilon^{2} w_{2}\left(x, T_{0}, T_{1} \ldots\right)+\ldots
$$

onde $\quad T_{j}=\varepsilon^{j} t, \quad D_{j}^{q}=\frac{d^{q}}{d T_{j}^{q}}, \quad \frac{d}{d t}=D_{0}+\varepsilon D_{1}+\varepsilon^{2} D_{2}+\ldots$, $\frac{d^{2}}{d t^{2}}=D_{0}^{2}+\varepsilon 2 D_{0} D_{1}+\varepsilon^{2}\left(D_{1}^{2}+2 D_{0} D_{2}\right)+\ldots$

Substitui-se a expressão $3.11 \mathrm{em} \mathrm{3.5.} \mathrm{Coletando} \mathrm{os} \mathrm{termos} \mathrm{em} \varepsilon, \varepsilon^{2}$ e $\varepsilon^{3}$ e aplicando as condições de solvabilidade encontra-se 3.9. Maiores detalhes em [51].

As equações de 3.1 a 3.10 apresentam a abordagem analítica. Um segundo cálculo para a determinação dos modos não-lineares é feito utilizando-se o método dos elementos finitos para a discretização do sistema estrutural. Em seguida, determinam-se os modos não-lineares utilizando a abordagem por variedades invariantes utilizando-se o modonlr (relações modais e equação do oscilador modal).

O modelo de elementos finitos utilizado é composto de 26 elementos retos não-lineares, como é possível observar na figura 3.2, que, também, apresenta as condições de contorno e a numeração dos graus de liberdade. Trata-se de um modelo de complexidade computacional média. O processamento deste modelo levou aproximadamente 17 horas utilizando um computador com 2GB de memória RAM e um processador de $1.6 \mathrm{GHz}$. 
Ressalta-se que a formulação dos modos não-lineares utilizando o $\mathrm{MEF}$ (Método dos Elementos Finitos) demanda considerável esforço algébrico e computacional, como pode ser observado em [6], [77] e [86]. Com relação aos modelos computacionais, a determinação dos modos não-lineares, ou seja, as relações modais e equação do oscilador modal, é a etapa que exige grandes tempos de processamento. Em contrapartida, o tempo de simulação do sistema é bastante pequeno, mesmo se tratando de um sistema não-linear. Os grandes tempos de processamento se devem ao fato de o programa trabalhar com tensores até quarta ordem e inversão da matriz de massa. Claramente, uma otmização do programa seria bastante interessante e útil, podendo constar na lista de trabalhos futuros.

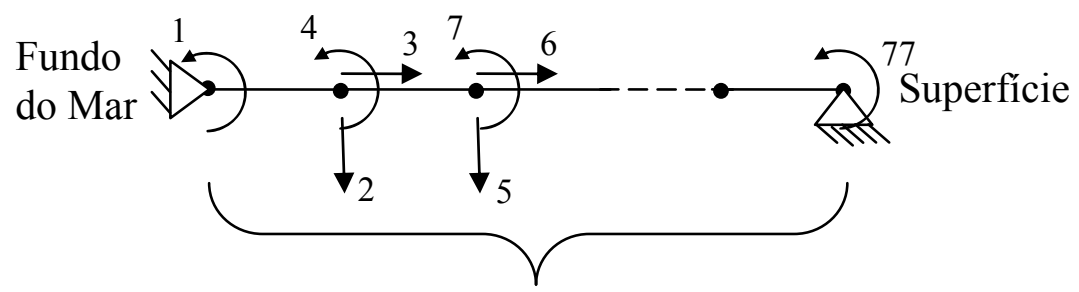

26 elementos

Figura 3.2 - Modelo do riser reto com 26 elementos e 77 graus de liberdade.

A tabela 3.1 apresenta as propriedades físicas do riser em estudo.

\begin{tabular}{|l|c|}
\hline Módulo de Young & $E=2,1 \times 10^{11} \mathrm{~N} / \mathrm{m}^{2}$ \\
\hline Comprimento & $\ell=1800 \mathrm{~m}$ \\
\hline Área da Seção Transversal & $A=1,1021 \times 10^{-2} \mathrm{~m}^{2}$ \\
\hline Momento de Inércia & $I=4,72143 \times 10^{-5} \mathrm{~m}^{4}$ \\
\hline Força Normal Inicial & $N_{0}=2 \times 10^{6} \mathrm{~N}$ \\
\hline $\begin{array}{l}\text { Massa por unidade de comprimento (água interna }+ \\
\text { massa adicionada) }\end{array}$ & $m=141,24 \mathrm{~kg} / \mathrm{m}$ \\
\hline
\end{tabular}

Tabela 3.1 - Propriedades físicas do riser.

Em todo este capítulo, será estudado o primeiro modo não-linear. Por simplicidade, omite-se, daqui por diante o índice $k$ que se refere ao modo desejado, ou seja, na ausência de índice, entende-se que $k=1$. Utilizando-se a equação 3.9, obtida 
através da abordagem analítica e os dados da tabela 3.1, encontra-se a seguinte equação do oscilador modal:

$$
\ddot{U}+4,32 \times 10^{-2} U+3,81 \times 10^{-5} U^{3}=0,
$$

onde $U$ é o deslocamento modal. Neste caso, $U$ foi escolhido de tal forma que ele é igual ao deslocamento transversal do ponto localizado na metade do comprimento do riser.

Através do M.E.F. e das variedades invariantes encontra-se a seguinte equação do oscilador modal:

$$
\begin{aligned}
& \ddot{U}+4,32 \times 10^{-2} U-1,91 \times 10^{-14} V+1,41 \times 10^{-15} U^{2}-3,97 \times 10^{-18} U V+2,98 \times 10^{-14} V^{2} \\
& -5,28 \times 10^{-5} U^{3}+1,53 \times 10^{-12} U^{2} V+6,31 \times 10^{-3} U V^{2}-1,18 \times 10^{-11} V^{3}=0
\end{aligned}
$$

onde $V$ é a velocidade modal, ou seja, $V=\dot{U}$.

Observando as equações dos osciladores modais 3.12 e 3.13 nota-se, em especial, a ausência do termo em $U V^{2}$ na equação 3.12 (analítica). Na equação 3.13, os coeficientes de $V, U^{2}, U V, V^{2}, U^{2} V$ e $V^{3}$ são, na verdade, nulos. Os coeficientes $10^{-12}, 10^{-14}, 10^{-15}$ e $10^{-18}$ são resíduos da solução numérica que, para estes coeficientes, tende a zero. De maneira simplificada, a equação 3.13 pode ser reescrita na forma:

$$
\ddot{U}+4,32 \times 10^{-2} U-5,28 \times 10^{-5} U^{3}+6,31 \times 10^{-3} U V^{2}=0
$$

Desta forma, fica evidente a principal diferença entre as duas abordagens, ou seja, o termo em $U V^{2}$, que a solução analítica não é capaz de capturar. O que se deve levar em consideração na apreciação das duas soluções é o valor das amplitudes. Em outras palavras, essas soluções são aproximações (uma analítica e a outra numérica) que têm suas validades restritas a determinados valores de amplitudes. Nos estudos 
que se seguem, verifica-se que a solução analítica apresenta bons resultados, em relação à resposta no tempo, até amplitudes da ordem de $15 \mathrm{~m}$ para este exemplo.

A integração das equações 3.12 e 3.14 foi feita através do método de RungeKutta. As figuras 3.3 e 3.4 mostram a resposta para os sistemas linear, M.E.F. e analítico. As condições iniciais (em $t=0$ ) adotadas foram $U_{0}=U(0)=10 \mathrm{~m}$ e $V_{0}=V(0)=0 \mathrm{~m} / \mathrm{s}$. Para todos os exemplos deste texto a velocidade modal inicial é nula. Portanto, nos próximos exemplos omite-se a velocidade modal inicial e subentende-se que ela valha zero. Os exemplos deste texto tratam, em sua mioria, de vibrações livres. Tais exemplos podem admitir amplitudes e velocidades excessivamente grandes que não correspondem aos valores de amplitude e velocidade encontrados rotineiramente em aplicações práticas. Estes valores excessivos têm a única finalidade de estudar os fenômenos não-lineares, a dinâmica não-linear dos risers e a robustez dos modelos matemáticos e computacionais aqui tratados.

Observando a figuras 3.3 fica evidente a boa concordância das duas soluções não-lineares (M.E.F. e analítica). Nota-se que ambas as soluções não-lineares apresentam uma freqüência maior que a solução linear. Entende-se, portanto, que as soluções não-lineares apontam um "hardening" (efeito da rigidez geométrica nãolinear) do sistema estrutural com o aumento da amplitude inicial. Este aumento da rigidez não é capturado pelo sistema linear. No entanto, as trajetórias no plano de fase apresentadas na figura 3.4, mostram a solução não-linear analítica próxima à solução linear. A solução não-linear analítica é representada, topologicamente, por um modo similar (ver [86]), ou seja, a solução linear e a solução não-linear analítica são similares topologicamente, mas possuem dinâmicas distintas. A similaridade topológica se deve ao fato de a solução não-linear analítica admitir que o primeiro modo normal não-linear de vibração seja senoidal. A solução não-linear utilizando MEF e variedaes invariantes não faz esta hipótese e, poratanto, sua forma modal não é similar.

Em razão desta simplificação adotada pela solução não-linear analítica, a equação do oscilador modal analítico apresenta um único termo não-linear, que é o termo em $U^{3}$. Enquanto na equação do oscilador modal via MEF, tem-se termos não- 
lineares em $U^{3}$ e em $U V^{2}$. Em ambos os casos esses termos tentam representar os mesmos fenômenos, porém no caso analítico esta tentativa é feita com um único termo e no caso numérico com dois. Claramente, a resposta analítica não está em condições de capturar os efeitos não-lineares oriundos da velocidade $V$, que estão associados aos efeitos inerciais introduzidos ne equação 2.79. No entanto, para valores de amplitude inferiores a $15 \mathrm{~m}$, estes efeitos não são significativos com relação à resposta no tempo.

Não obstante, as figuras 3.5 e 3.6 apresentam resultados para uma amplitude inicial de $15 \mathrm{~m}$. Novamente, as soluções não-lineares seguem concordando, porém, no final do intervalo de integração (fig. 3.5), já é possível notar uma pequena discrepância. A tendência de aumento da rigidez continua presente nas soluções nãolineares e, obviamente, não é percebida pela solução linear. Verifica-se também no diagrama de fase o distanciamento da solução não-linear via MEF das outras duas soluções, evidenciando que a solução via MEF possui uma topologia distinta e, portanto, não-similar. 


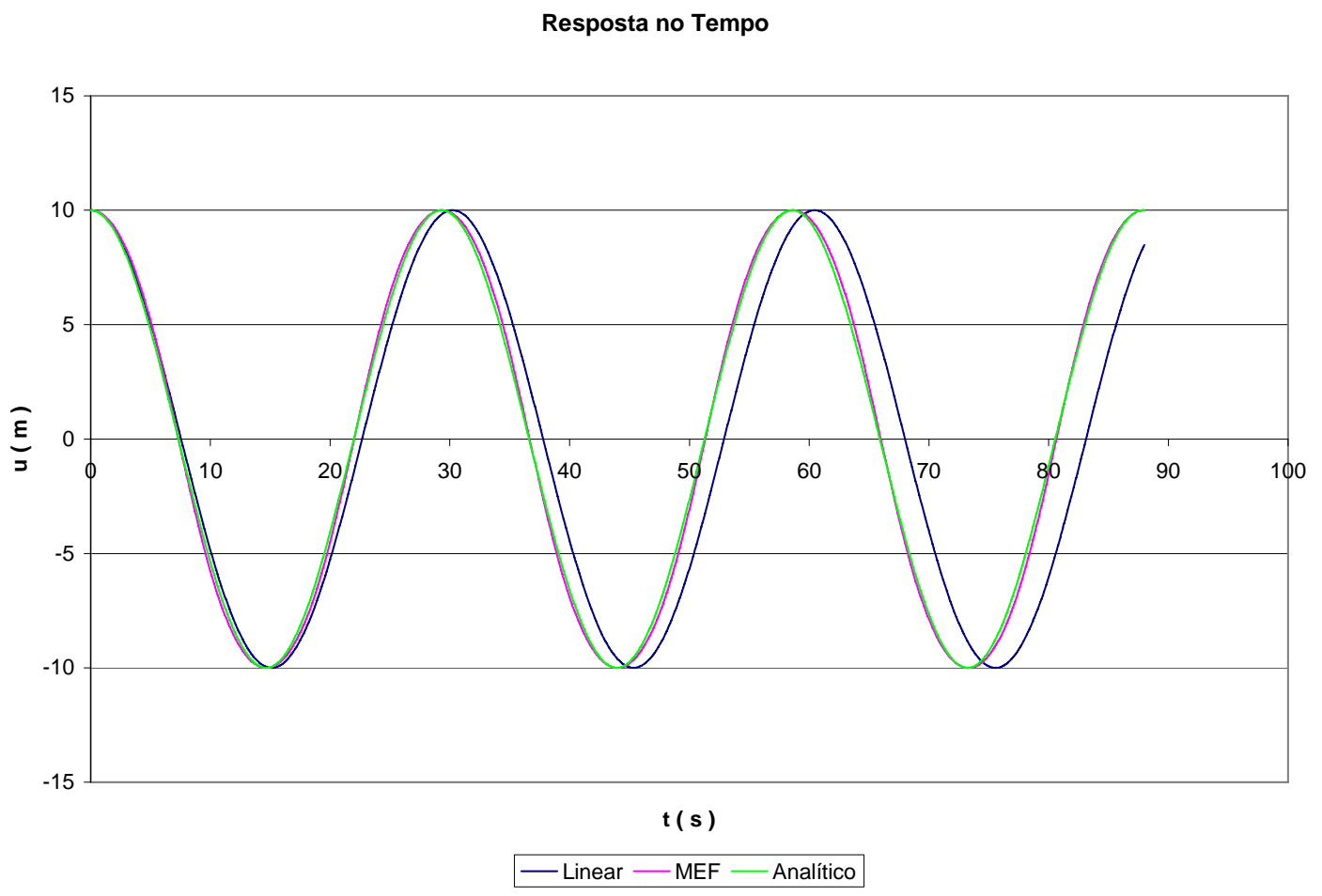

Figura 3.3 - Resposta no tempo para o primeiro modo; amplitude inicial de $10 \mathrm{~m}$. Períodos: 30.23 s (linear); 29.33 s (MEF); $29.30 \mathrm{~s} \mathrm{(analítico)}$

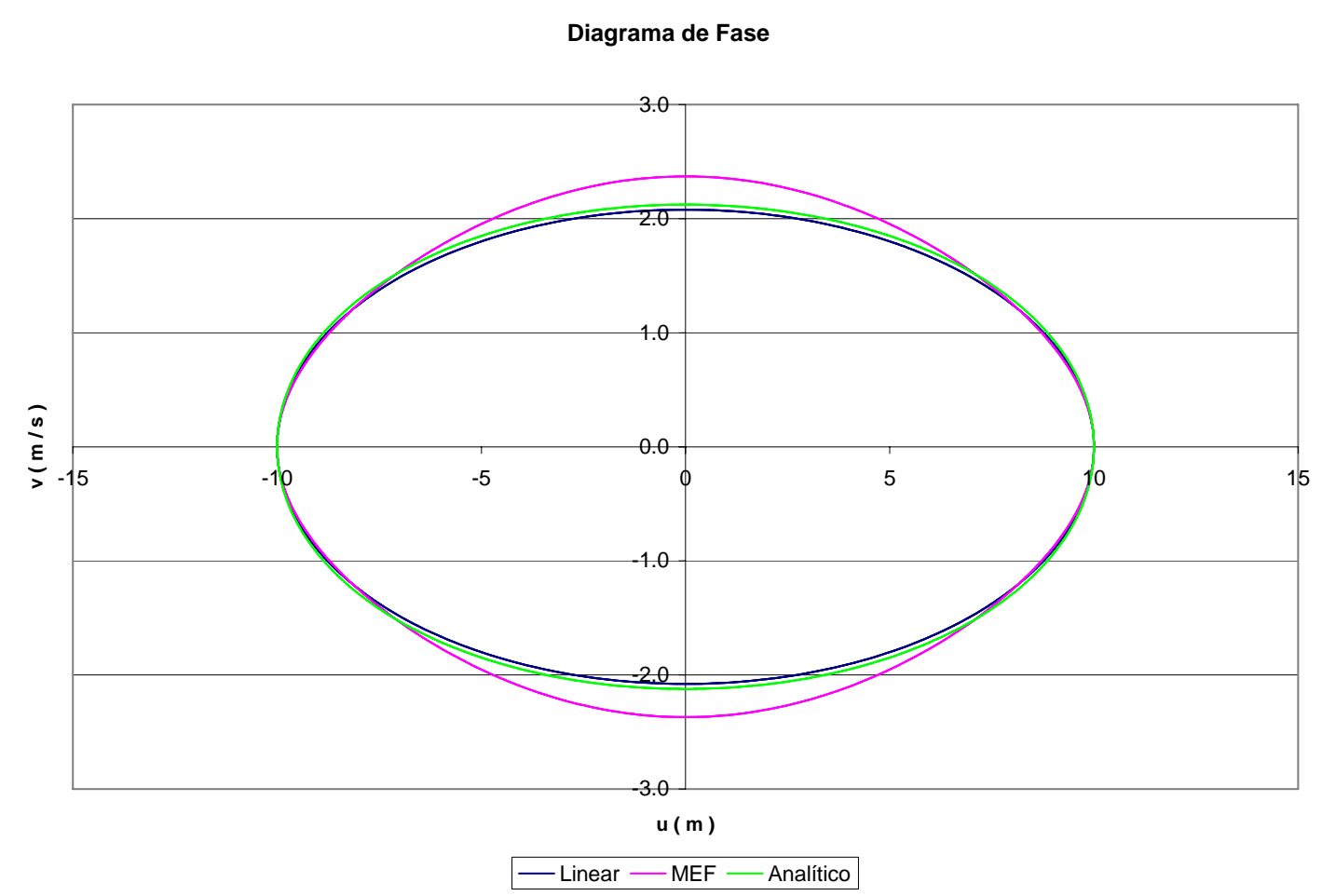

Figura 3.4 - Diagrama de fase para o primeiro modo; amplitude inicial de $10 \mathrm{~m}$. 
Resposta no Tempo

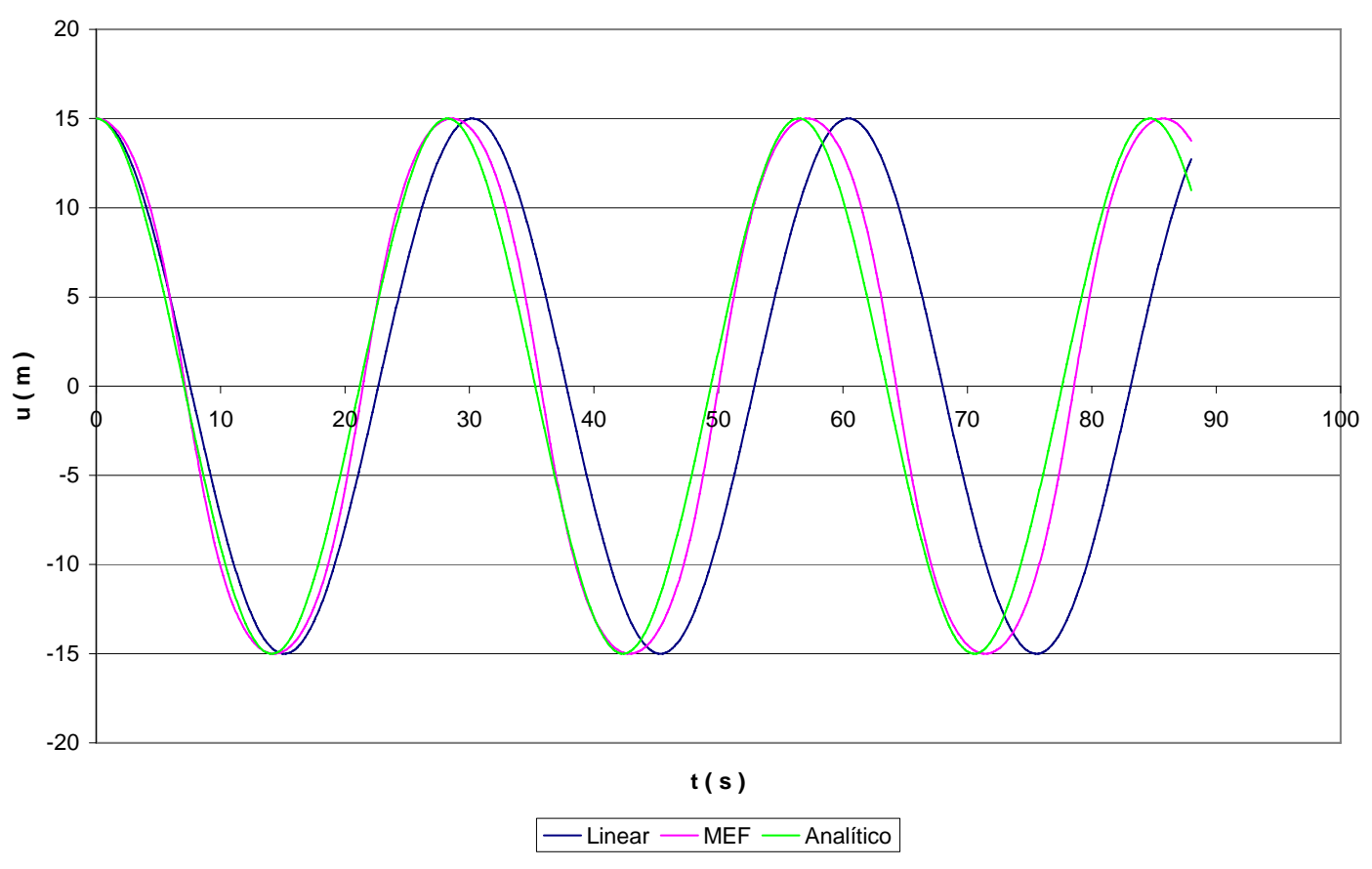

Figura 3.5 - Resposta no tempo para o primeiro modo; amplitude inicial de $15 \mathrm{~m}$. Períodos: 30.23 s (linear); 28.57 s (MEF); 28.23 s (analítico).

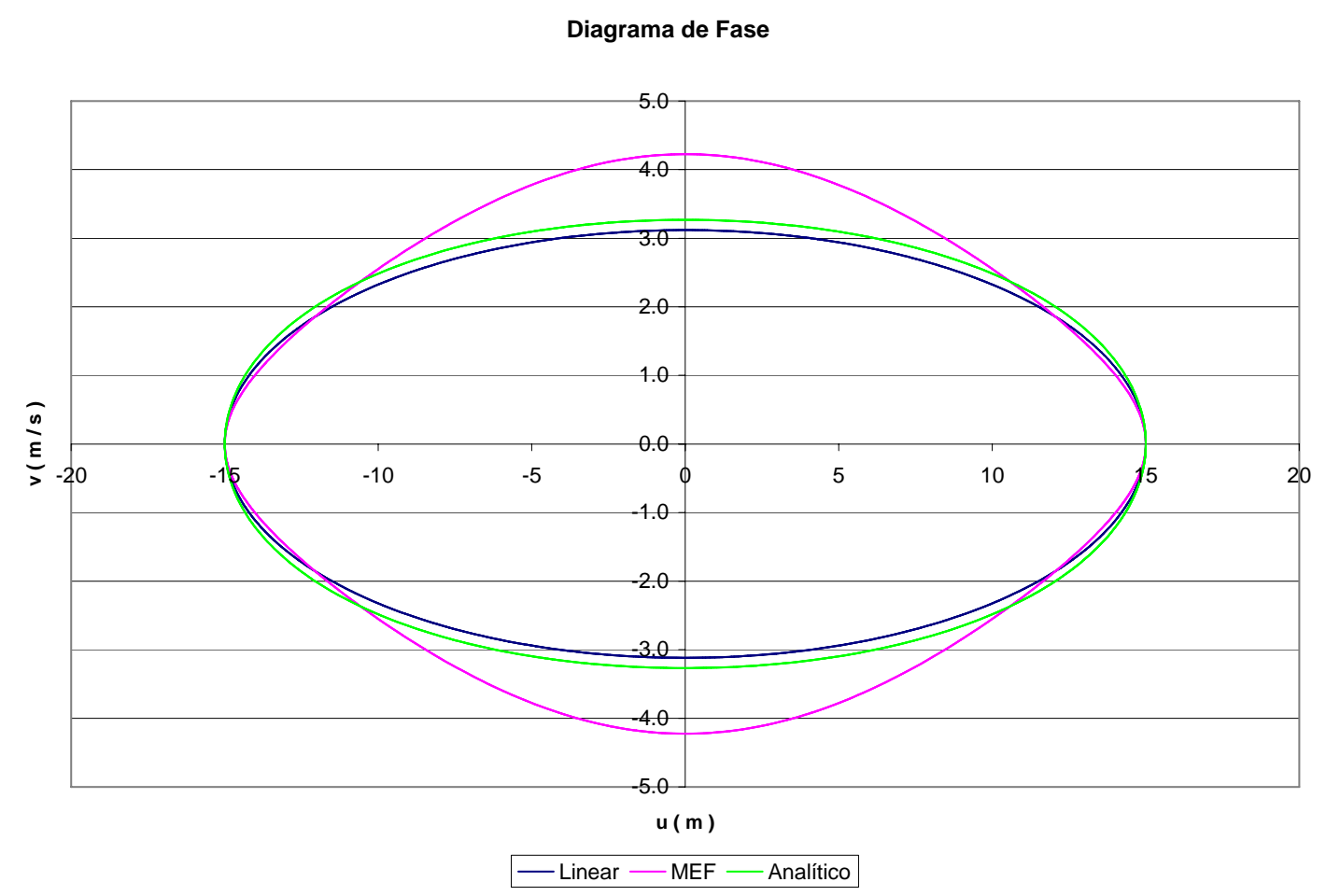

Figura 3.6 - Diagrama de fase para o primeiro modo; amplitude inicial de $15 \mathrm{~m}$. 
Com o intuito de investigar o aumento da rigidez, as figuras 3.7 e 3.8 apresentam as resposta para uma amplitude inicial de $20 \mathrm{~m}$. Neste caso, apesar de as soluções não-lineares continuarem capturando o enrijecimento da estrutura, é possível observar significativa diferença entre elas. Isto se deve fortemente à presença do termo em $U V^{2}$ na solução pelo M.E.F.. Este termo é influenciado pela velocidade ao quadrado que ganha mais importância quanto maior for a amplitude inicial.

Tomando-se uma amplitude inicial de $25 \mathrm{~m}$, obtêm-se as figuras 3.9 e 3.10 . Nelas se observa o completo descolamento entres as soluções não-lineares. Além disso, nota-se que a solução não-linear pelo M.E.F. apresenta uma freqüência inferior à freqüência do sistema linear. Curiosamente, este sistema estrutural inverte sua tendência de "hardening", passando a apresentar "softening". Este resultado mostra a importância do termo em $U V^{2}$. Quanto maiores as amplitudes, e conseqüentemente maiores as velocidades, maior é a influência deste termo. Esta influência também pode ser apreciada observando o diagrama de fase (fig. 3.10), que apresenta uma alteração significativa. Nota-se que a não-similaridade do modo de vibração nãolinear via MEF, juntamente com a influência do termo em $U V^{2}$, pode ser responsável pela a inversão da tendência de "hardening" na medida em este modo é capaz de sofrer a influência de modos superiores como, por exemplo, o terceiro modo. Esta influência pode diminuir a rigidez a flexão do sistema levando à tendência de "softening" observada nas figuras 3.9 e 3.10 . 
Resposta no Tempo

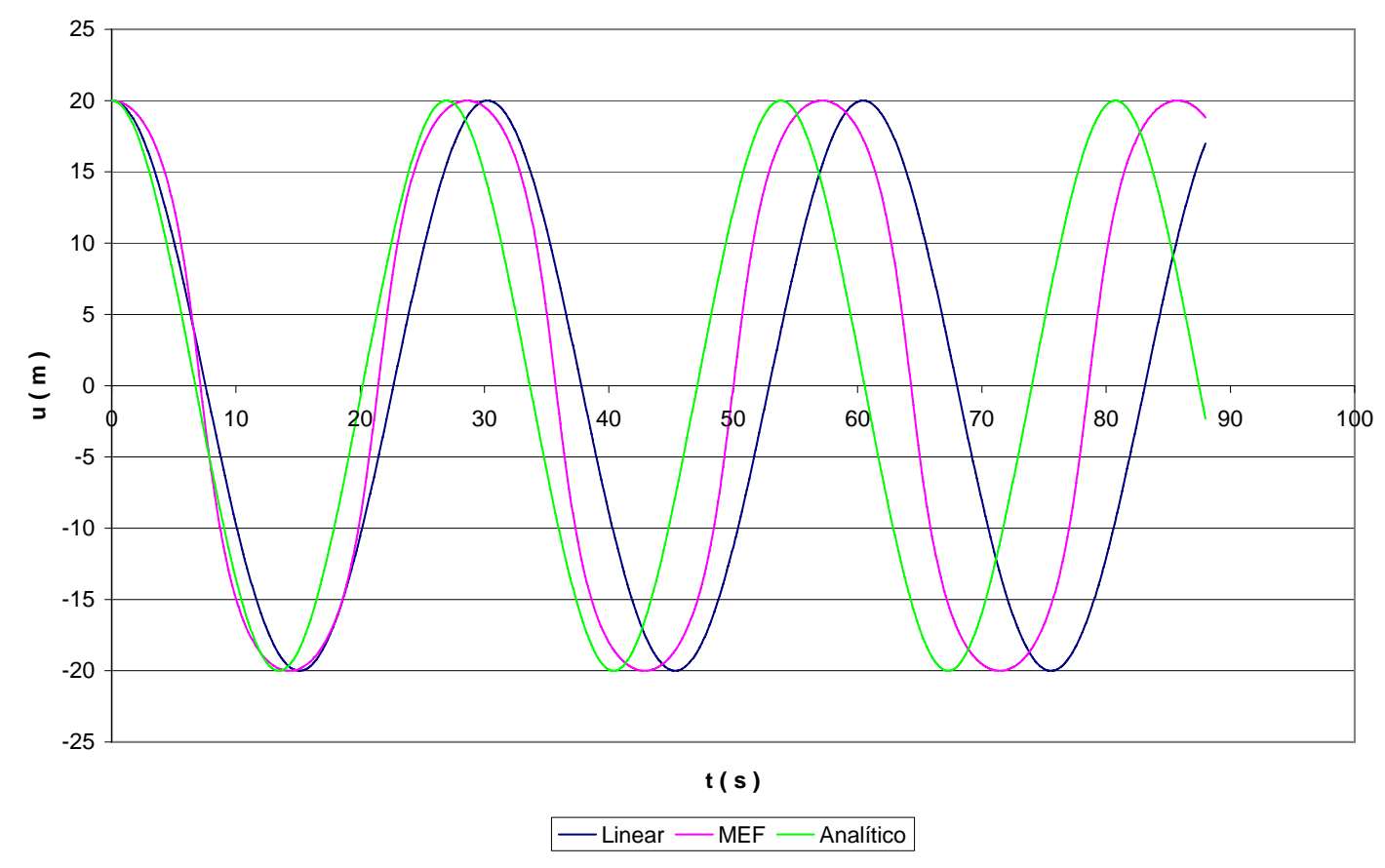

Figura 3.7 - Resposta no tempo para o primeiro modo; amplitude inicial de $20 \mathrm{~m}$. Períodos: 30.23 s (linear); 28.58 s (MEF); 26.93 s (analítico).

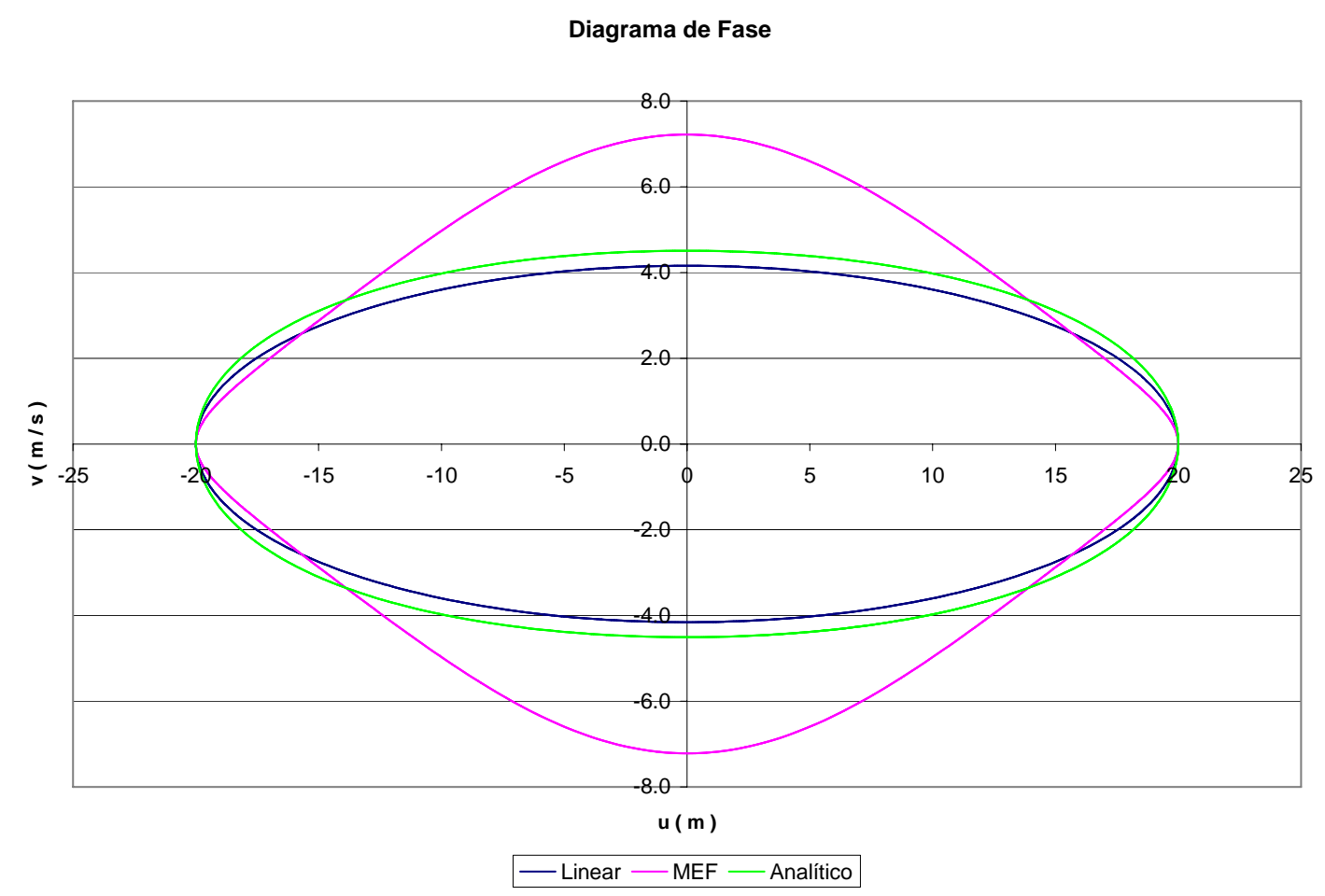

Figura 3.8 - Diagrama de fase para o primeiro modo; amplitude inicial de $20 \mathrm{~m}$. 


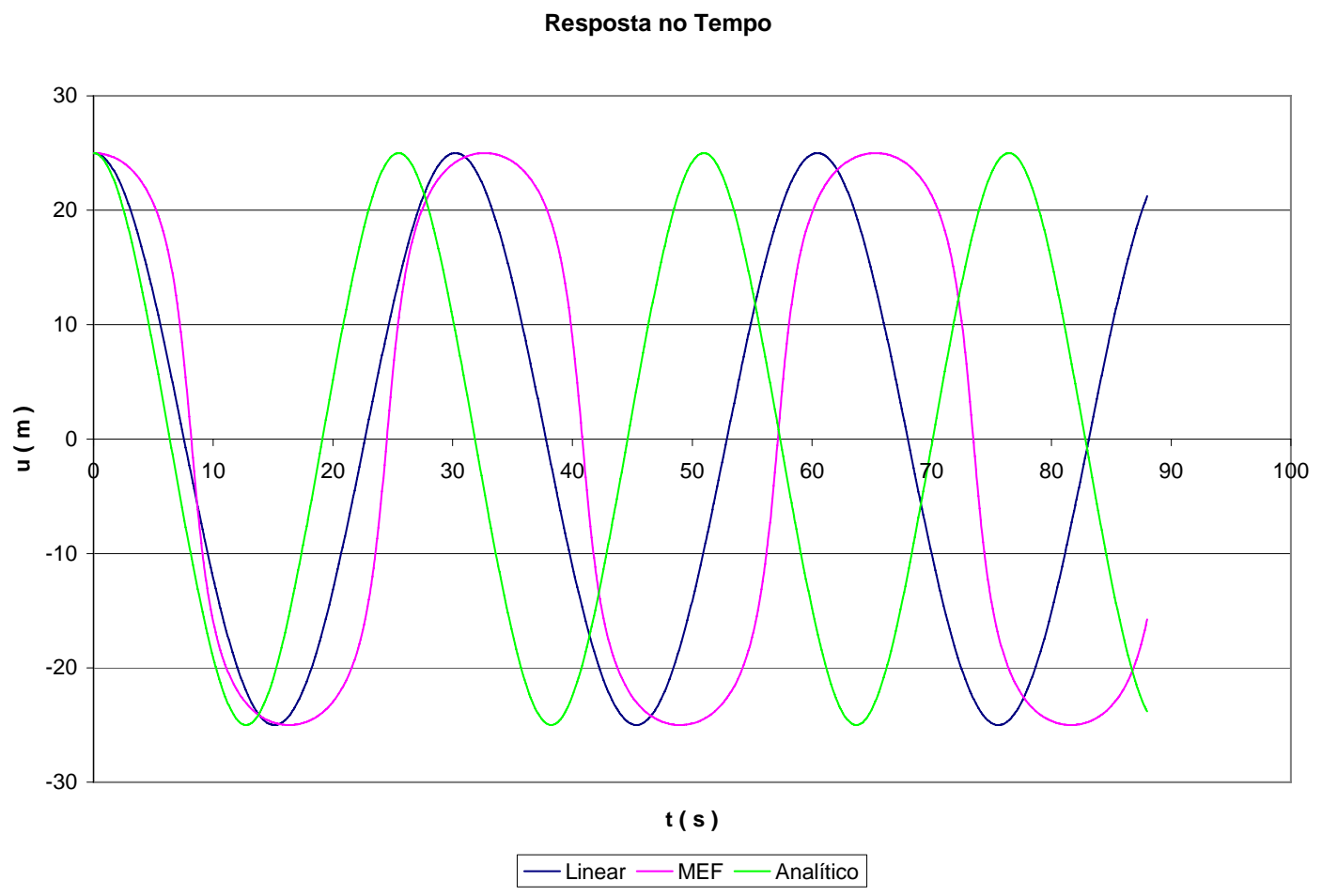

Figura 3.9 - Resposta no tempo para o primeiro modo; amplitude inicial de $25 \mathrm{~m}$. Períodos: $30.23 \mathrm{~s}$ (linear); $32.65 \mathrm{~s}$ (MEF); $25.50 \mathrm{~s}$ (analítico).

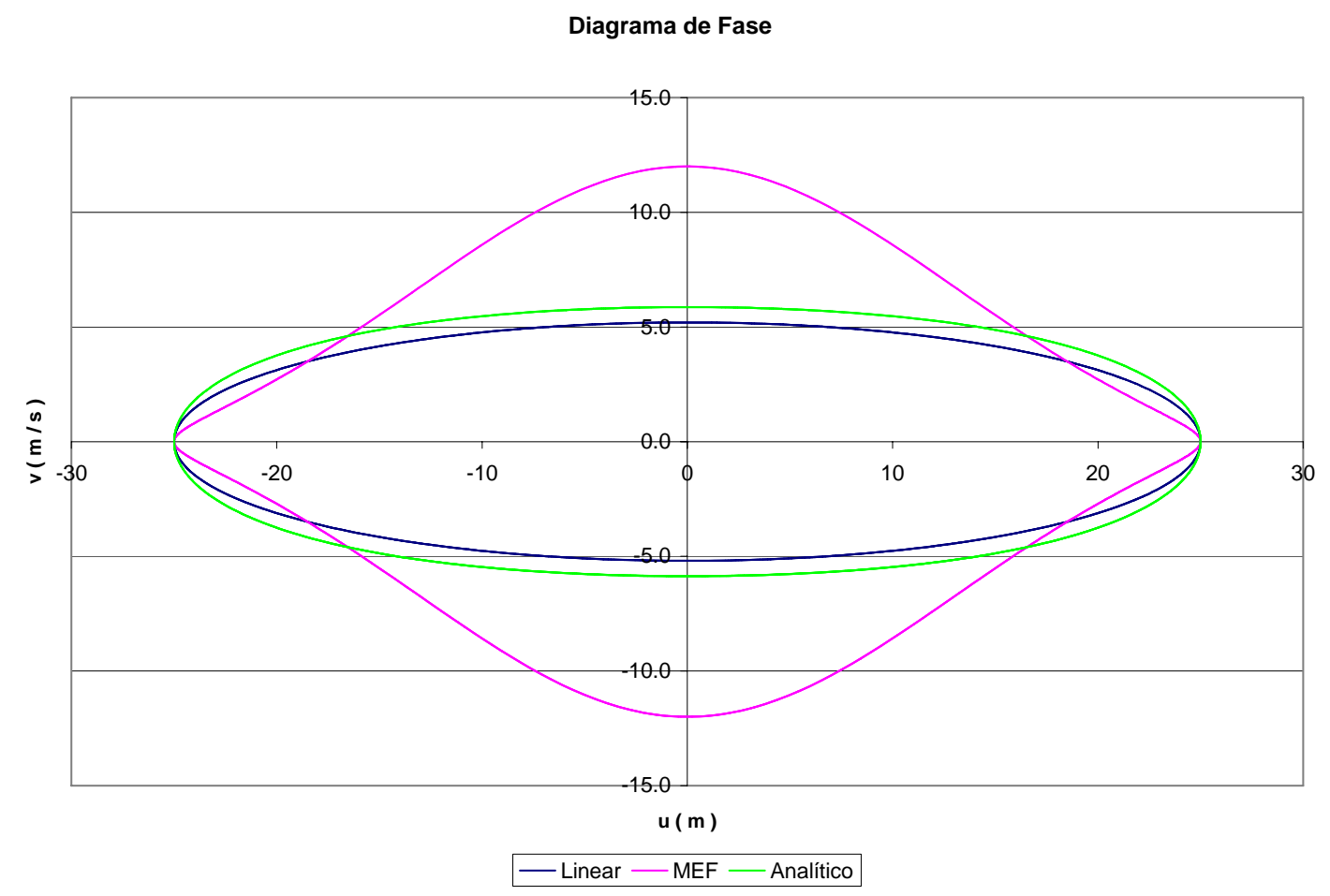

Figura 3.10 - Diagrama de fase para o primeiro modo; amplitude inicial de $25 \mathrm{~m}$. 


\subsection{Com Peso Próprio}

Nesta seção, analisa-se um riser com as mesmas propriedades físicas, geometria e condições de contorno da seção anterior. Porém, neste caso, considera-se seu peso próprio $p$. Admite-se que força normal inicial, na seção transversal junto à superfície, tenha valor $\mathrm{N}_{0}$. A força normal inicial ao longo do riser varia linearmente segundo a expressão:

$$
N(x, 0)=N(0,0)-p x
$$

onde $x$ é o eixo longitudinal com sentido da superfície para o fundo do mar.

A figura 3.11 mostra um arranjo esquemático do sistema estrutural em questão. Nesta seção, apresentam-se, através de uma abordagem analítica, as equações de movimento não-linear do riser reto considerando-se a influência do peso próprio.

Considerando as equações 3.1, 3.2 e 3.3, e, utilizando o princípio de Hamilton (maiores detalhes em Pars [68] e Meirovitch [54]), a seguinte equação de movimento transversal pode ser escrita (ver Mazzilli et al [51]):

$$
m \ddot{w}+E I w^{I V}-E A\left[\frac{u_{\ell}-u_{0}}{\ell}+\frac{1}{2 \ell} \int_{0}^{\ell} w^{\prime 2} d x+\frac{p}{E A}\left(\frac{\ell}{2}-x\right)\right] w^{\prime \prime}+p w^{\prime}=0 .
$$

Em qualquer instante, a força normal ao longo do riser pode ser calculada por:

$$
N(x, t)=N_{0}(t)-p x=-\frac{E A u_{0}}{\ell}+p\left(\frac{\ell}{2}-x\right)+\frac{E A}{2 \ell} \int_{0}^{\ell} w^{\prime 2} d x
$$



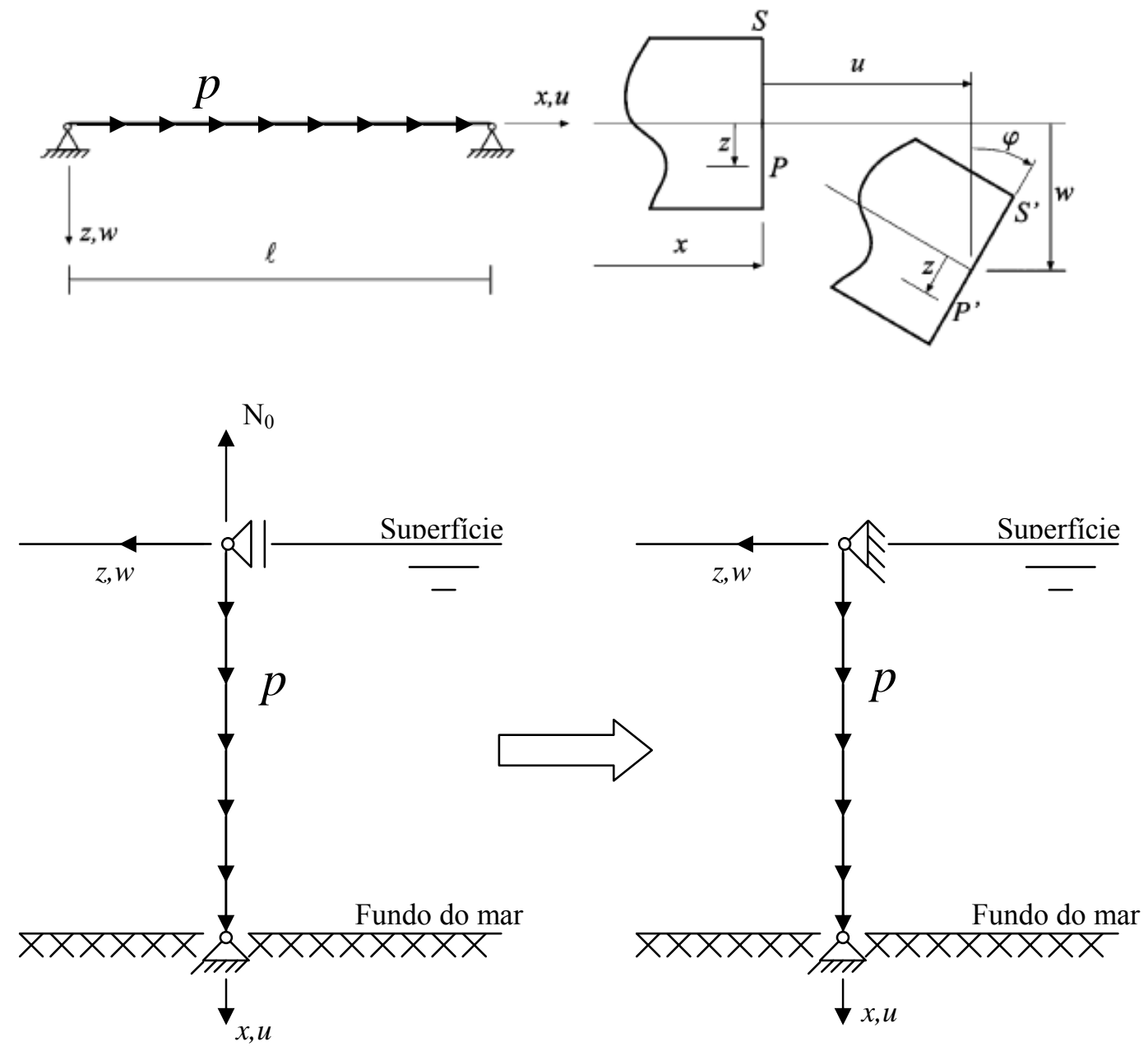

Figura 3.11 - Modelo esquemático com peso próprio.

Observando as equações 3.16 e 3.17, é possível reescrever 3.16 na forma:

$$
m \ddot{w}+E I w^{i v}-N(x, t) w^{\prime \prime}+p w^{\prime}=0 .
$$

Admite-se, agora, uma aproximação. Com o intuito de eliminar a dependência espacial de $N(x, t)$, toma-se o valor médio da força normal $\bar{N}$ ao longo do riser e substitui-se $\bar{N}$ na equação 3.18 para se chegar a:

$$
m \ddot{w}+E I w^{I V}-\bar{N} w^{\prime \prime}-\frac{E A}{2 \ell} w^{\prime \prime} \int_{0}^{\ell} w^{\prime} d x+p w^{\prime}=0,
$$


onde

$$
\bar{N}=N_{0}(0)-\frac{p \ell}{2} .
$$

O deslocamento axial pode ser escrito como:

$$
u(x, t)=-\frac{\bar{N}(\ell-x)}{E A}+\frac{p x(\ell-x)}{2 E A}-\frac{1}{2} \int_{0}^{x}\left(\frac{d w}{d \xi}\right)^{2} d \xi+\frac{x}{2 \ell} \int_{0}^{\ell} w^{\prime 2} d x
$$

A equação 3.19 é retomada na forma:

$$
\ddot{w}+\alpha w^{I V}-\beta w^{\prime \prime}-\mu w^{\prime \prime} \int_{0}^{\ell} w^{\prime 2} d x+\varepsilon^{3} \gamma w^{\prime}=0,
$$

onde

$$
\alpha=\frac{E I}{m} ; \quad \beta=\frac{\bar{N}}{m} ; \quad \mu=\frac{E A}{2 m \ell} ; \quad \varepsilon^{3} \gamma=\frac{p}{m}, \quad 0<\varepsilon<1 .
$$

A solução de 3.22, utilizando o método das múltiplas escalas, pode ser escrita na forma:

$$
w(x, t)=\varepsilon w_{1}\left(x, T_{0}, T_{1} \ldots\right)+\varepsilon^{2} w_{2}\left(x, T_{0}, T_{1} \ldots\right)+\ldots
$$

onde as seguintes escalas de tempo são assumidas:

$$
T_{j}=\varepsilon^{j} t
$$

$\operatorname{com} j=1,2,3$.

Os seguintes operadores diferenciais são intoduzidos: 


$$
\begin{aligned}
& D_{j}^{q}=\frac{d^{q}}{d T_{j}^{q}}, \\
& \frac{d}{d t}=D_{0}+\varepsilon D_{1}+\varepsilon^{2} D_{2}+\ldots, \\
& \frac{d^{2}}{d t^{2}}=D_{0}^{2}+\varepsilon 2 D_{0} D_{1}+\varepsilon^{2}\left(D_{1}^{2}+2 D_{0} D_{2}\right)+\ldots .
\end{aligned}
$$

Substituindo as expressões 3.24, 3.25 e 3.26 em 3.22 e coletando os de mesma ordem $\varepsilon^{j}$, é possível encontrar equações diferenciais cujas soluções e condições de solvabilidade permitem a determinação dos modos não-lineares.

\section{Solução de Ordem $\varepsilon$}

Coletando os termos em $\varepsilon$, vem:

$$
D_{0}^{2} w_{1}+\alpha w_{1}^{I V}-\beta w_{1}^{\prime \prime}=0
$$

As condições de contorno são:

$$
w_{1}(0)=w_{1}(\ell)=w_{1}^{\prime \prime}(0)=w_{1}^{\prime \prime}(\ell)=0
$$

A solução $w_{1}$ pode ser escrita na forma:

$w_{1}\left(x, T_{0}, T_{1} \ldots\right)=\sum_{k} w_{1 k}\left(x, T_{0}, T_{1} \ldots\right), \quad w_{1 k}\left(x, T_{0}, T_{1} \ldots\right)=A_{1 k}\left(T_{0}, T_{1} \ldots\right) \sin \frac{k \pi x}{\ell}$

Substituindo 3.29 em 3.27 vem:

$$
D_{0}^{2} A_{1 k}+\omega_{k}^{2} A_{1 k}=0, \quad \omega_{k}=\frac{k \pi}{\ell} \sqrt{\beta+\frac{\alpha k^{2} \pi^{2}}{\ell^{2}}}
$$

A solução de 3.30 pode ser escrita na forma complexa: 
Solução de Ordem $\varepsilon^{2}$

Coletando os termos em $\varepsilon^{2}$, vem:

$$
D_{0}^{2} w_{2 k}+\alpha w_{2 k}^{I V}-\beta w_{2 k}^{\prime \prime}=-2 D_{0} D_{1} w_{1 k}
$$

As condições de solvabilidade levam a $D_{1} Y_{k}=0$, então $Y_{k}=Y_{k}\left(T_{2}, \ldots\right)$. Portanto, a solução $w_{2 k}$ já está contida em $w_{1 k}$.

\section{Solução de Ordem $\varepsilon^{3}$}

Coletando os termos em $\varepsilon^{3}$, vem:

$$
\begin{aligned}
& D_{0}^{2} w_{3 k}+\alpha w_{3 k}^{I V}-\beta w_{3 k}^{\prime \prime}=-2 D_{0} D_{2} w_{1 k}+\mu w_{1 k}^{\prime \prime} \int_{0}^{\ell} w_{1 k}^{\prime 2} d x \\
& =\left[-2 i \omega_{k} e^{i \omega_{k} T_{0}} D_{2} Y_{k}-\Lambda_{k}\left(Y_{k}^{3} e^{i 3 \omega_{k} T_{0}}+3 Y_{k}^{2} \bar{Y}_{k} e^{i \omega_{k} T_{0}}\right)\right] \sin \frac{k \pi x}{\ell},
\end{aligned}
$$

onde

$$
\Lambda_{k}=\left(\frac{\mu \ell}{2}\right) \frac{k^{4} \pi^{4}}{\ell^{4}}=\left(\frac{E A}{4 m}\right) \frac{k^{4} \pi^{4}}{\ell^{4}} .
$$

As condições de solvabilidade levam a:

$$
-2 i \omega_{k} D_{2} Y_{k}-3 \Lambda_{k} Y_{k}^{2} \bar{Y}_{k}=0
$$

Para determinar a solução de $3.35, Y_{k}$ é escrito na forma: 


$$
Y_{k}=\frac{1}{2} a_{k} e^{i \theta_{k}}, \quad a_{k}, \theta_{k} \in \mathfrak{R}
$$

Pode-se determinar que:

$$
a_{k}=a_{k}\left(T_{3}\right), \quad \theta_{k}=\theta_{0 k}\left(T_{3}\right)+\frac{3 \Lambda_{k}}{8 \omega_{k}} a_{k}^{2} T_{2}
$$

Considerando as soluções $w_{1 k}, w_{2 k}$ e $w_{3 k}$, o k-ésimo modo não-linear é definido no tempo por:

$$
\begin{aligned}
w_{k}(x, t) & =\left(\varepsilon a_{k}\right)\left\{\cos \left(\Omega_{k} t+\theta_{0 k}\right)+\frac{\Lambda_{k}\left(\varepsilon a_{k}\right)^{2}}{32 \omega_{k}^{2}} \cos 3\left(\Omega_{k} t+\theta_{0 k}\right)\right\} \sin \frac{k \pi x}{\ell}+O\left(\varepsilon^{4}\right), \\
u_{k}(x, t) & =-\frac{k \pi}{8 \ell}\left(\varepsilon a_{k}\right)^{2}\left\{\cos \left(\Omega_{k} t+\theta_{0 k}\right)+\frac{\Lambda_{k}\left(\varepsilon a_{k}\right)^{2}}{32 \omega_{k}^{2}} \cos 3\left(\Omega_{k} t+\theta_{0 k}\right)\right\}^{2} \sin \frac{2 k \pi x}{\ell} \\
& \cong-\frac{k \pi}{16 \ell}\left(\varepsilon a_{k}\right)^{2}\left[1+\cos 2\left(\Omega_{k} t+\theta_{0 k}\right)\right] \sin \frac{2 k \pi x}{\ell}+O\left(\varepsilon^{4}\right),
\end{aligned}
$$

onde $\varepsilon a_{k}$ é a amplitude do k-ésimo modo linear associado.

A relação entre a freqüência não-linear e a amplitude pode ser escrita na forma:

$$
\Omega_{k}=\omega_{k}\left[1+\frac{3 \Lambda_{k}}{8 \omega_{k}^{2}}\left(\varepsilon a_{k}\right)^{2}\right]
$$

\section{Construção da Variedade Invariante}

Um modo não-linear pode ser caracterizado escrevendo-se os deslocamentos e velocidades generalizados como funções de duas variáveis modais escolhidas ad hoc. Com este intuito, consideram-se as seguintes variáveis modais: 


$$
U_{k}(t)=w_{k}(\bar{x}, t), \quad V_{k}(t)=\dot{U}_{k}(t)
$$

onde $\bar{x}$ é tal que,

$$
\sin \left(\frac{k \pi}{\ell}\right) \bar{x}=1 .
$$

Os deslocamentos do k-ésimo modo podem ser escritos em função das variáveis modais:

$$
w_{k}(x, t)=F_{1 k}^{w}(x) U_{k}(t), \quad u_{k}(x, t)=F_{3 k}^{u}(x) U_{k}^{2}(t)
$$

Observando a expressão 3.40, as velocidades podem ser escritas em termos das variáveis modais como:

$$
\dot{w}_{k}=G_{2 k}^{w} V_{k}, \quad \dot{u}_{k}=G_{4 k}^{u} U_{k} V_{k},
$$

onde

$$
F_{1 k}^{w}(x)=G_{2 k}^{w}=\sin \frac{k \pi x}{\ell} \text { e } F_{3 k}^{u}(x)=\frac{1}{2} G_{4 k}^{u}=-\frac{k \pi}{8 \ell} \sin \frac{2 k \pi x}{\ell} .
$$

A equação do oscilador modal pode ser escrita como:

$$
\ddot{U}_{k}+\omega_{k}^{2} U_{k}+\varepsilon^{2} \Lambda_{k} U_{k}^{3}=0
$$

Como na seção anterior, estuda-se o modelo de riser com peso próprio pela abordagem analítica (descrita acima) e pela abordagem via M.E.F. e variedades invariantes (utilizando-se o programa de computador modonlr). O modelo de elementos finitos utilizado é o mesmo da figura 3.2, que possui 26 elementos e 77 graus de liberdade. 
O modelo de elementos finitos utilizado é o mesmo utilizado na seção anterior, apresentado na figura 3.2, com a adição do peso próprio. A tabela 3.2 apresenta as propriedades físicas do riser.

\begin{tabular}{|l|c|}
\hline Módulo de Young & $E=2,1 \times 10^{11} \mathrm{~N} / \mathrm{m}^{2}$ \\
\hline Comprimento & $\ell=1800 \mathrm{~m}$ \\
\hline Área da Seção Transversal & $A=1,1021 \times 10^{-2} \mathrm{~m}^{2}$ \\
\hline Momento de Inércia & $I=4,72143 \times 10^{-5} \mathrm{~m}^{4}$ \\
\hline Força Normal Inicial (no topo) & $N_{0}=2 \times 10^{6} \mathrm{~N}$ \\
\hline Força Normal Inicial (na base) & $N_{\ell}=6,914 \times 10^{5} \mathrm{~N}$ \\
\hline $\begin{array}{l}\text { Massa por unidade de comprimento (água interna }+ \\
\text { massa adicionada) }\end{array}$ & $m=141,24 \mathrm{~kg} / \mathrm{m}$ \\
\hline Peso submerso do riser por unidade de comprimento & $p=727 \mathrm{~N} / \mathrm{m}$ \\
\hline
\end{tabular}

Tabela 3.2 - Propriedades físicas do riser.

Como já mencionado na seção anterior, estuda-se neste capítulo o primeiro modo; por simplicidade, omite-se o índice $k$ que se refere ao modo desejado, ou seja, na ausência de índice entende-se que $k=1$. Utilizando-se a equação 3.45 , obtida através da abordagem analítica e os dados da tabela 3.1, encontra-se a seguinte equação do oscilador modal para o riser reto com peso próprio:

$$
\ddot{U}+2,70 \times 10^{-2} U+3,81 \times 10^{-5} U^{3}=0,
$$

onde $U$ é o deslocamento modal. Neste caso, $U$ foi escolhido de tal forma que ele seja igual ao deslocamento transversal do ponto localizado na metade do comprimento do riser.

Comparando-se as equações 3.12 e 3.46 nota-se que os coeficientes de $U^{3}$ nas duas equações são iguais. Isto se deve ao fato do termo em $U^{3}$ representar a influência da rigidez à flexão, que se mantém inalterada do primeiro exemplo para o segundo. Já o coeficiente em $U^{2}$ se altera, uma vez que a freqüência linear é significativamente influenciada pela rigidez geométrica do sistema. Tal rigidez é influenciada pela variação da força normal, introduzida pela consideração do peso próprio do riser $p$. 
Através do M.E.F. e das variedades invariantes encontra-se a seguinte equação do oscilador modal para o riser reto com peso próprio:

$$
\begin{aligned}
& \ddot{U}+2,70 \times 10^{-2} U-1,85 \times 10^{-14} V+7,01 \times 10^{-15} U^{2}+1,09 \times 10^{-15} U V-2,73 \times 10^{-13} V^{2}, \\
& +2,52 \times 10^{-4} U^{3}-8,19 \times 10^{-14} U^{2} V-2,27 \times 10^{-2} U V^{2}+1,01 \times 10^{-12} V^{3}=0
\end{aligned}
$$

onde $V$ é a velocidade modal, ou seja, $V=\dot{U}$.

Novamente, nota-se a ausência do termo em $U V^{2}$ na equação 3.46 (analítica). Na equação 3.47, os coeficientes de $V, U^{2}, U V, V^{2}, U^{2} V$ e $V^{3}$ são desprezíveis. De maneira simplificada, a equação 3.47 pode ser reescrita na forma:

$$
\ddot{U}+2,70 \times 10^{-2} U+2,52 \times 10^{-4} U^{3}-2,27 \times 10^{-2} U V^{2}=0
$$

Comparando-se as equações 3.13 e 3.48 nota-se a inversão dos sinais dos termos em $U^{3}$ e $U V^{2}$. A equação 3.13 é o oscilador modal não-linear para o riser reto sem peso próprio e a equação 3.48 é o oscilador modal não-linear para o riser reto com peso próprio, ambas encontradas utilizando MEF e variedades invariantes. Apesar da inversão de sinais, ambos os termos em $U^{3}$ e $U V^{2}$ estão aclopados para representar o mesmo fenômeno da dinâmica não-linear de risers. O termo em $U V^{2}$ está relacionado aos efeitos inerciais introduzidos na equação 2.79 e, é análogo ao termo $\theta \dot{\theta}^{2}$, encontrado no estudo do pêndulo duplo (ver [16], [18], [33], [55], [94], [95] e [96]), que, também, representa a influência dos efeitos inerciais na dinâmica do sistema. Estes efeitos se tornam significativos quando considerado o peso próprio, pois este altera a rigidez do sistema ao longo do comprimento do riser. Assim, o período de vibração também se altera ao longo da estrutura dando origem às “travelling waves", ou seja, os pontos nodais se deslocam no tempo.

O modelo de elementos finitos é capaz de considerar a variação da força normal ao longo do riser e, portanto, leva a uma solução mais adequada do que a solução analítica apresentada neste texto. Uma formulação analítica que considera a 
variação da força normal e apresenta resultados coerentes com os resultados encontrados nesta tese, é apresentada em [53].

Adimensionalizando a equação 3.48 da seguinte forma:

$$
\hat{U}=\frac{U}{\ell} \quad \hat{V}=\frac{V}{\ell},
$$

e admitindo-se $\hat{U}(0)=0,01$ encontra-se o diagrama de fase apresentado na figura 3.12 que concorda com os resultados apresentados por Mazzilli e Wiercigroch [53].

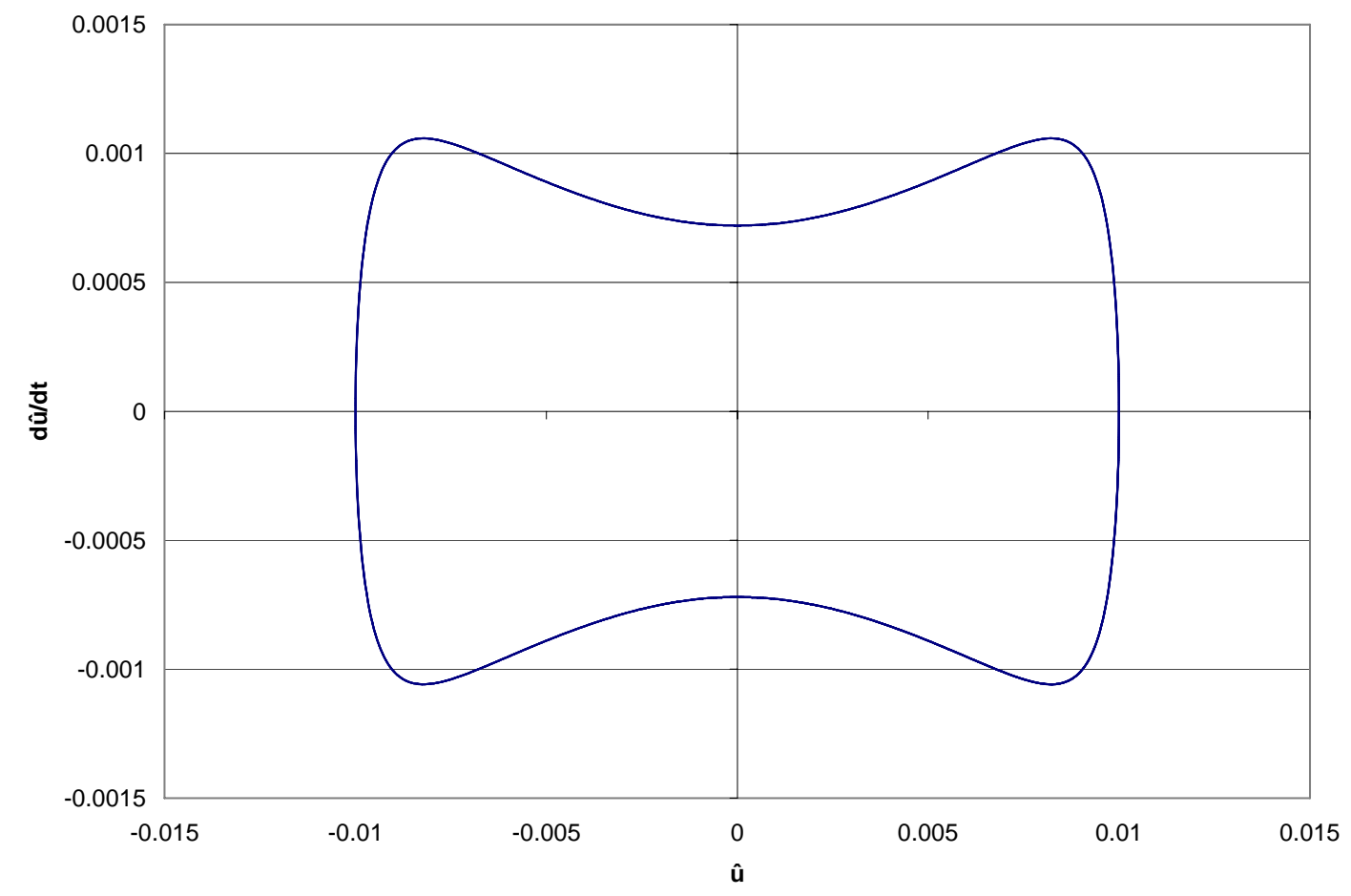

Figura 3.12 - Diagrama de fase adimensional para a resposta via MEF.

A integração das equações 3.46 e 3.48 foi feita através do método de RungeKutta. As figuras 3.13 e 3.14 mostram a resposta para os sistemas linear, M.E.F. e analítico. As condições iniciais (em $t=0$ ) adotadas foram $U_{0}=U(0)=10 \mathrm{~m} \mathrm{e}$ $V_{0}=V(0)=0 \mathrm{~m} / \mathrm{s}$. Para todos os exemplos deste capítulo a velocidade modal inicial é nula. Portanto, nos próximos exemplos omite-se a velocidade modal inicial e subentende-se que ela valha zero. 
Observando as figuras 3.13 e 3.14, verifica-se que a solução não-linear analítica não coincide, como esperado, com a solução linear. No entanto, a solução não-linear analítica também diverge da solução não-linear via M.E.F.. A freqüência encontrada pela solução não-linear analítica é maior que a freqüência do sistema linear. Contrariamente, a solução não-linear via M.E.F. encontra uma freqüência menor que a freqüência linear. Enquanto a solução analítica prevê um aumento da rigidez do sistema estrutural, a solução via M.E.F. aponta uma redução da rigidez desse mesmo sistema.

Essa diferença é explicada pelo termo em $U V^{2}$, que inexiste na solução analítica, no entanto exerce um papel importante na solução via M.E.F.. Tomando-se, por exemplo, na figura 3.14 (diagrama de fase), o intervalo $-5 m \leq U \leq 5 m$, observase uma redução significativa da velocidade modal para a solução pelo M.E.F. Tal redução implica aumento do período e conseqüente redução da freqüência. Apesar de o termo em $U^{3}$ levar ao aumento da freqüência, o termo em $U V^{2}$ supera essa tendência devido aos valores significativos de amplitude e velocidade. 


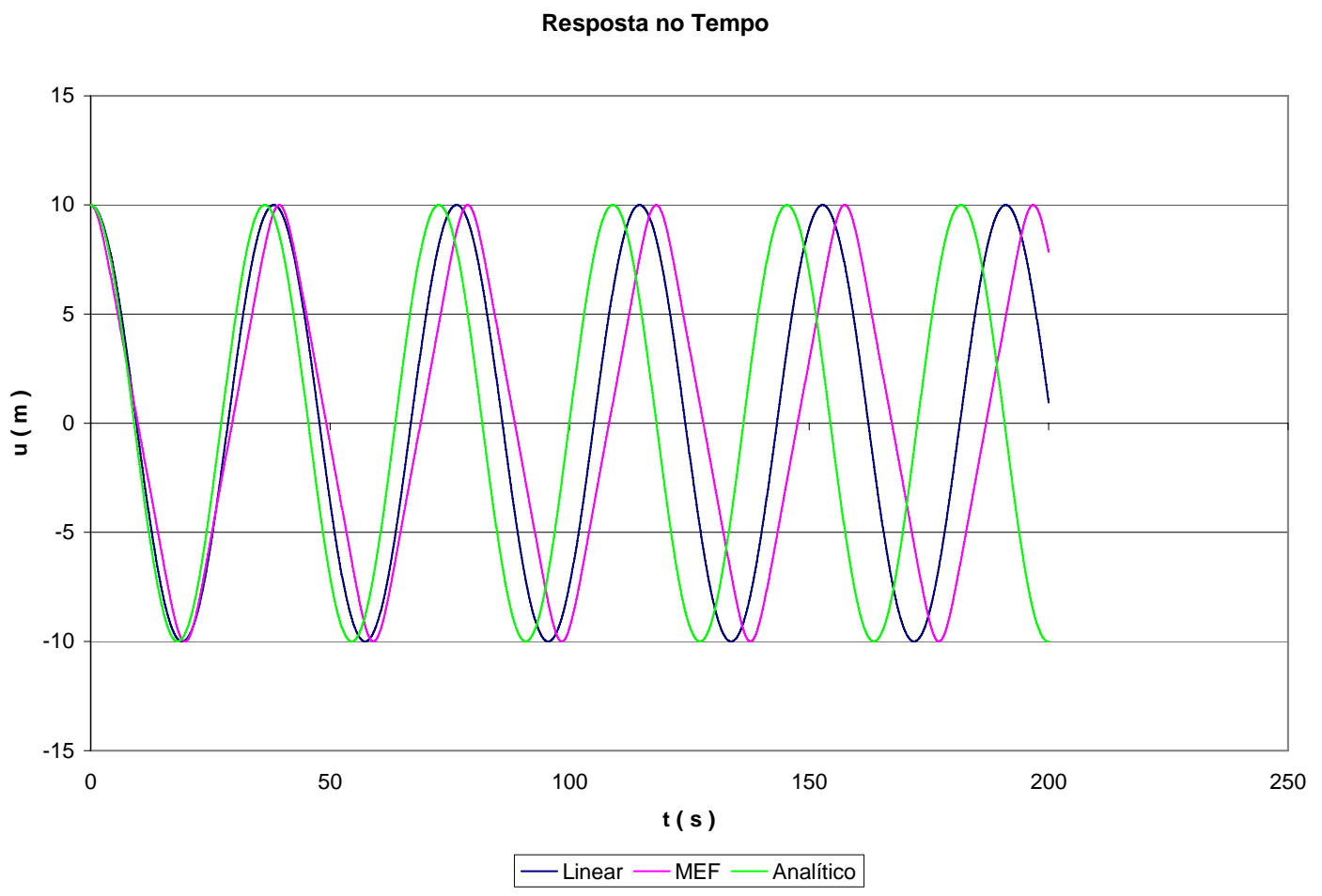

Figura 3.13 - Resposta no tempo para o primeiro modo; amplitude inicial de $10 \mathrm{~m}$. Períodos: 38,21 s (linear); 39,36 s (MEF); 36,35 s (analítico)

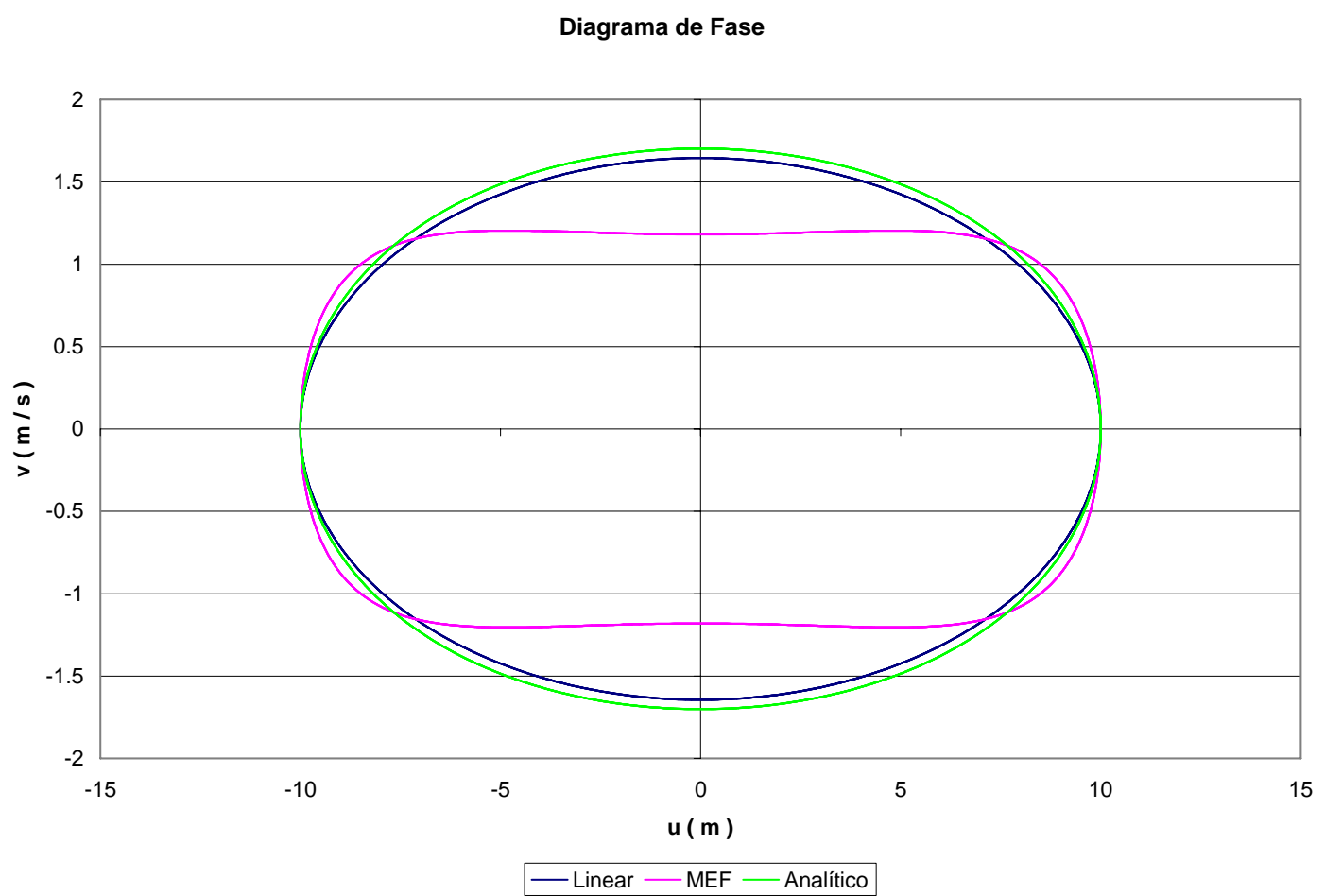

Figura 3.14 - Diagrama de fase para o primeiro modo; amplitude inicial de $10 \mathrm{~m}$. 


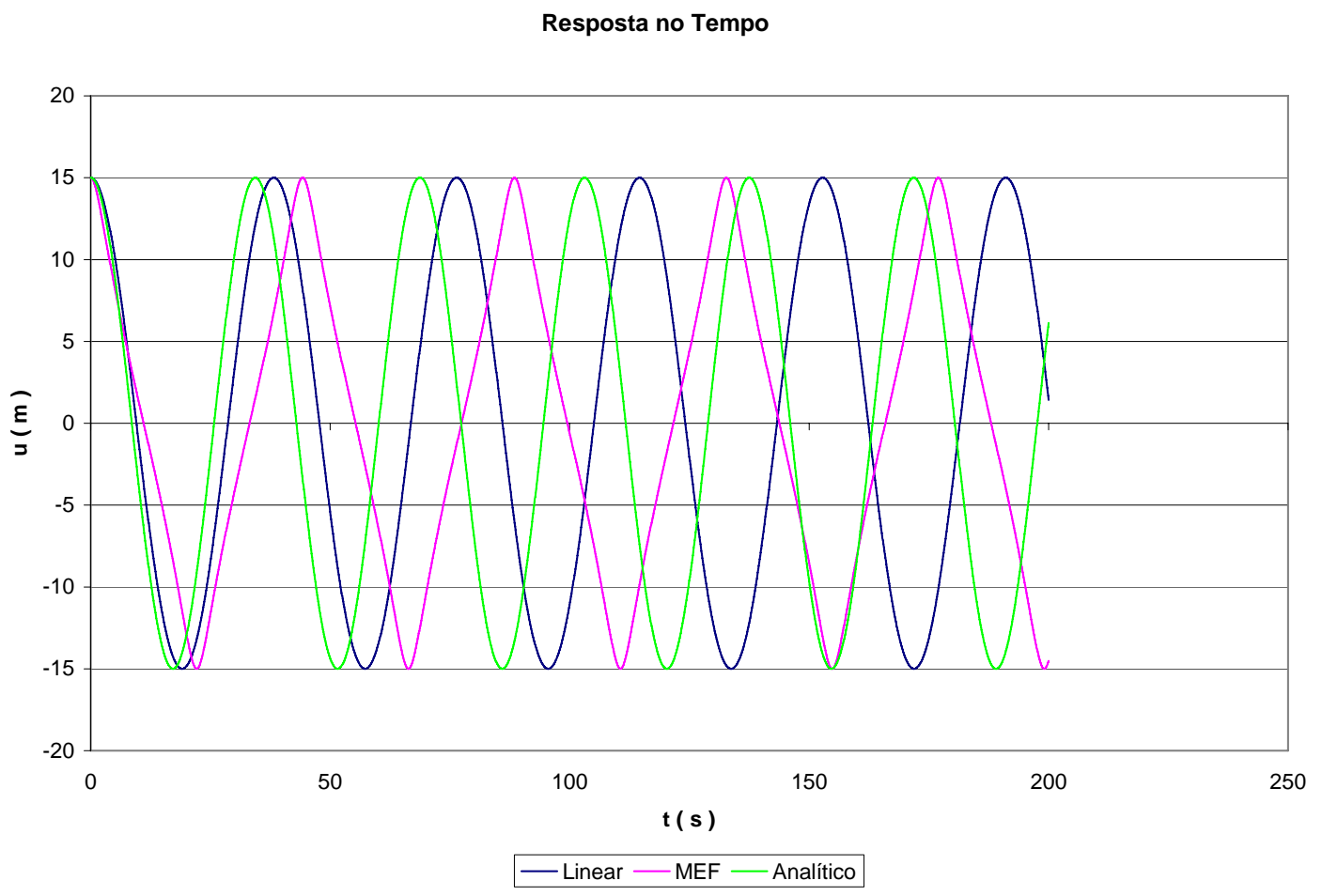

Figura 3.15 - Resposta no tempo para o primeiro modo; amplitude inicial de $15 \mathrm{~m}$. Períodos: 38,21 s (linear); 44,24 s (MEF); 34,36 s (analítico)

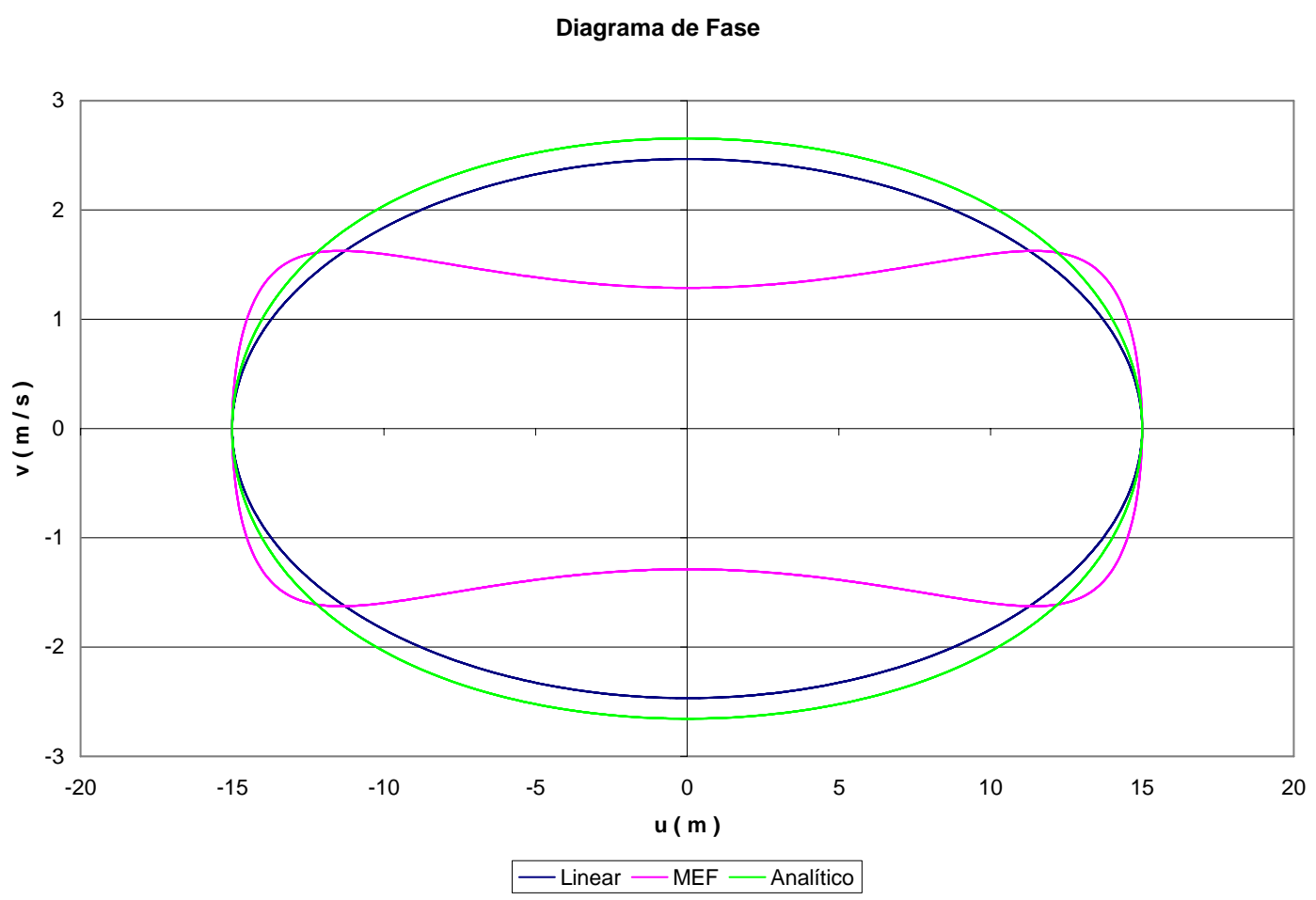

Figura 3.16 - Diagrama de fase para o primeiro modo; amplitude inicial de $15 \mathrm{~m}$. 
Não obstante, as figuras 3.15 e 3.16 apresentam resultados para uma amplitude inicial de $15 \mathrm{~m}$. Novamente, as soluções não-lineares discordam. A tendência de aumento da rigidez continua presente na solução analítica e o contrário na solução via M.E.F.

Com intuito de investigar o comportamento das duas soluções não-lineares, as figuras 3.17 e 3.18 apresentam as respostas para uma amplitude inicial de $20 \mathrm{~m}$. Neste caso, a solução não-linear analítica diverge substancialmente da linear, apresentando significativo enrijecimento. Em oposição, a solução via M.E.F. mostra queda significativa da freqüência e, portanto, diminuição da rigidez. Isto se deve fortemente, como dito anteriormente, à presença do termo em $U V^{2}$ na solução pelo M.E.F.

Uma situação ainda mais extrema é encontrada tomando-se uma amplitude inicial de $25 \mathrm{~m}$. Os resultados podem ser observados nas figuras 3.19 e 3.20. No diagrama de fase apresentado na figura 3.20 nota-se o forte "estrangulamento" da resposta do sistema não-linear via M.E.F. Para este nível de amplitude é possível notar, no caso da solução via M.E.F., a influência do terceiro modo linear no primeiro modo não-linear, ver figura 3.21. Vale ressaltar que até este ponto foram estudados apenas modos não-lineares normais e não multi-modos. No entanto, verifica-se a capacidade do primeiro modo normal ser influenciado pelo terceiro modo. Diante desta constatação, relembra-se que este sistema estrutural apresenta ressonância interna com relação racional entre as freqüências dos modos lineares e relações típicas que deflagram acoplamentos não-lineares, como as não-linearidades cúbicas e o acoplamento entre primeiro e terceiro modos não-lineares. Tal propriedade motiva o estudo de multi-modos a seguir. 


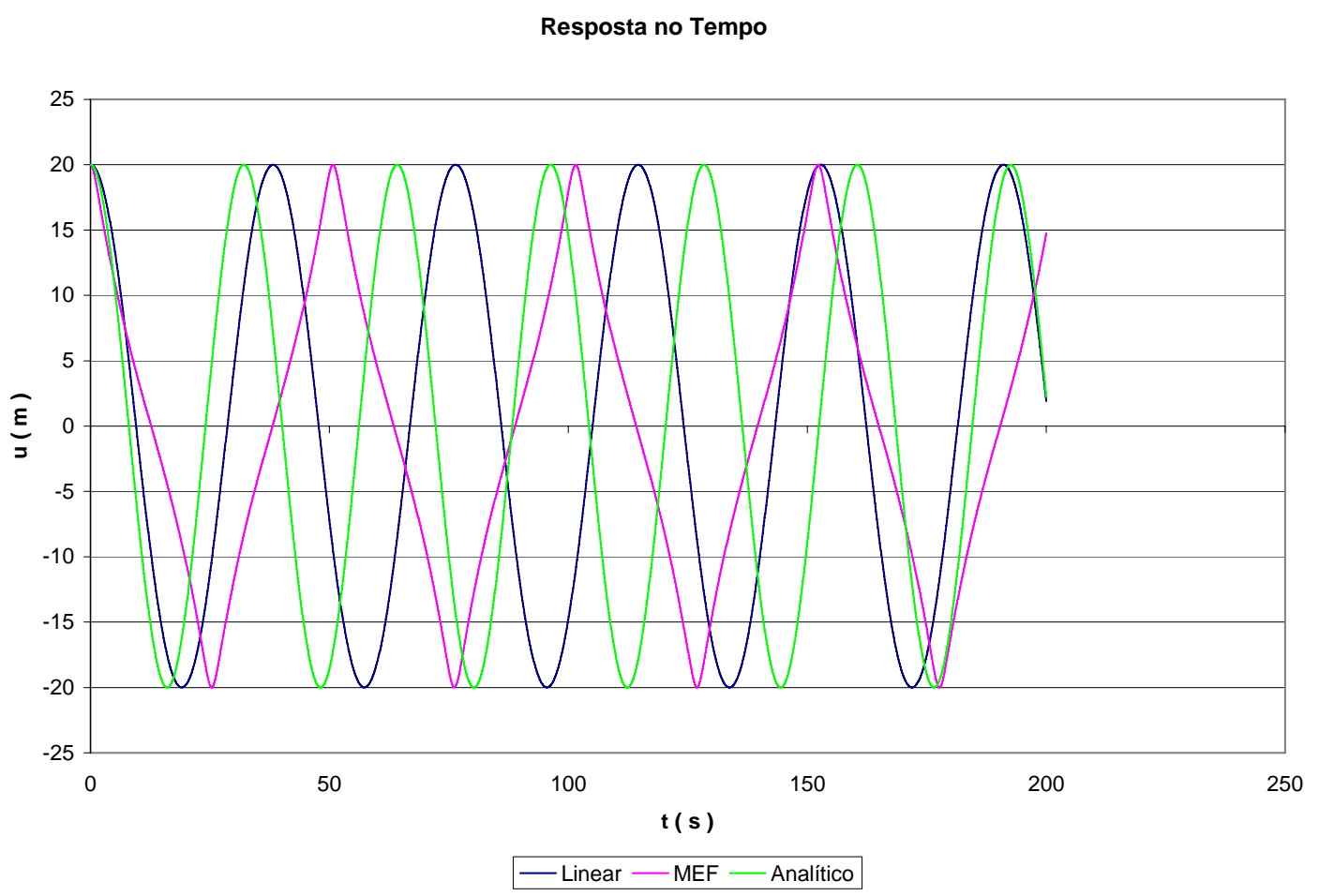

Figura 3.17 - Resposta no tempo para o primeiro modo; amplitude inicial de $20 \mathrm{~m}$. Períodos: 38,21 s (linear); 50,77 s (MEF); 32,10 s (analítico)

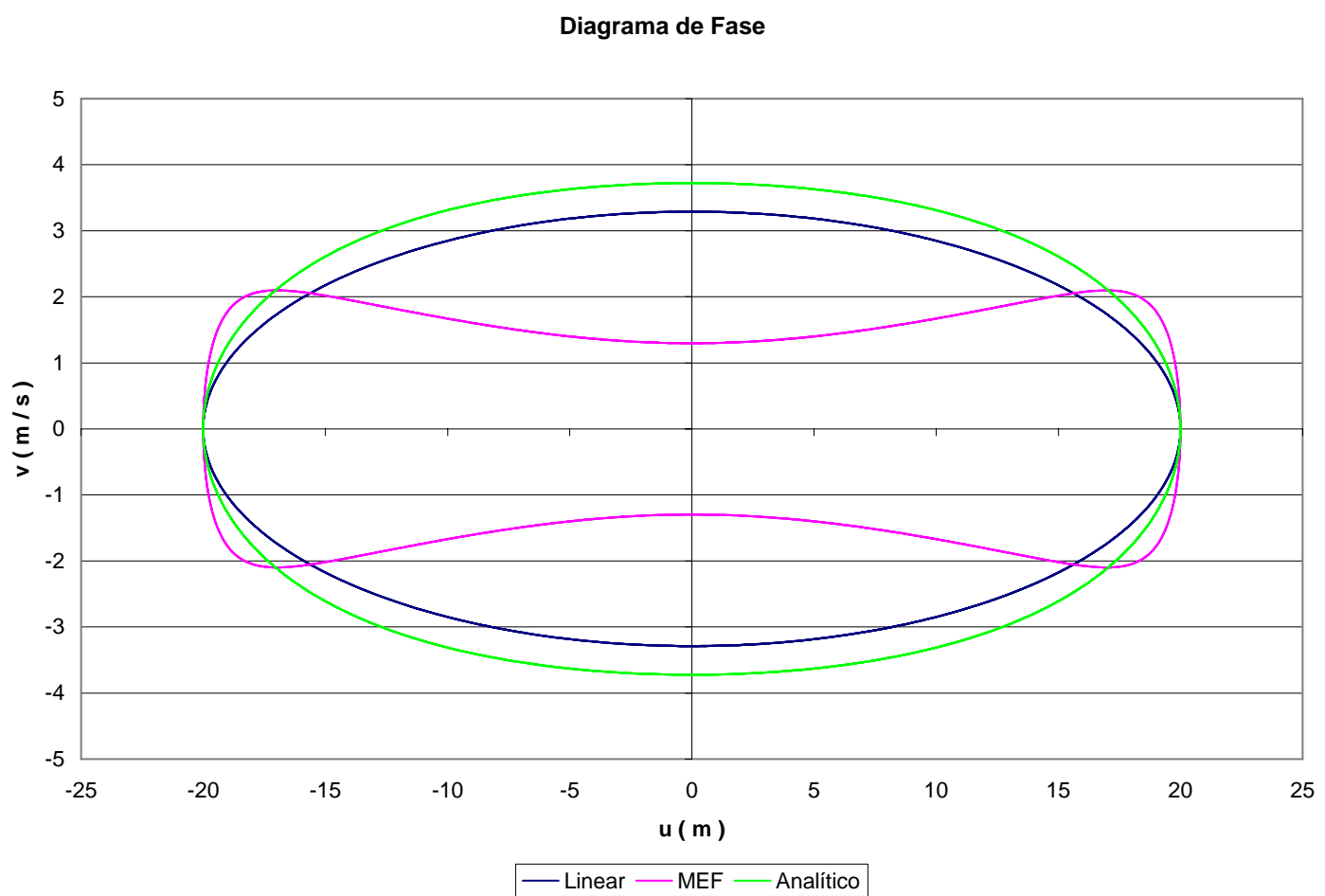

Figura 3.18 - Diagrama de fase para o primeiro modo; amplitude inicial de $20 \mathrm{~m}$. 


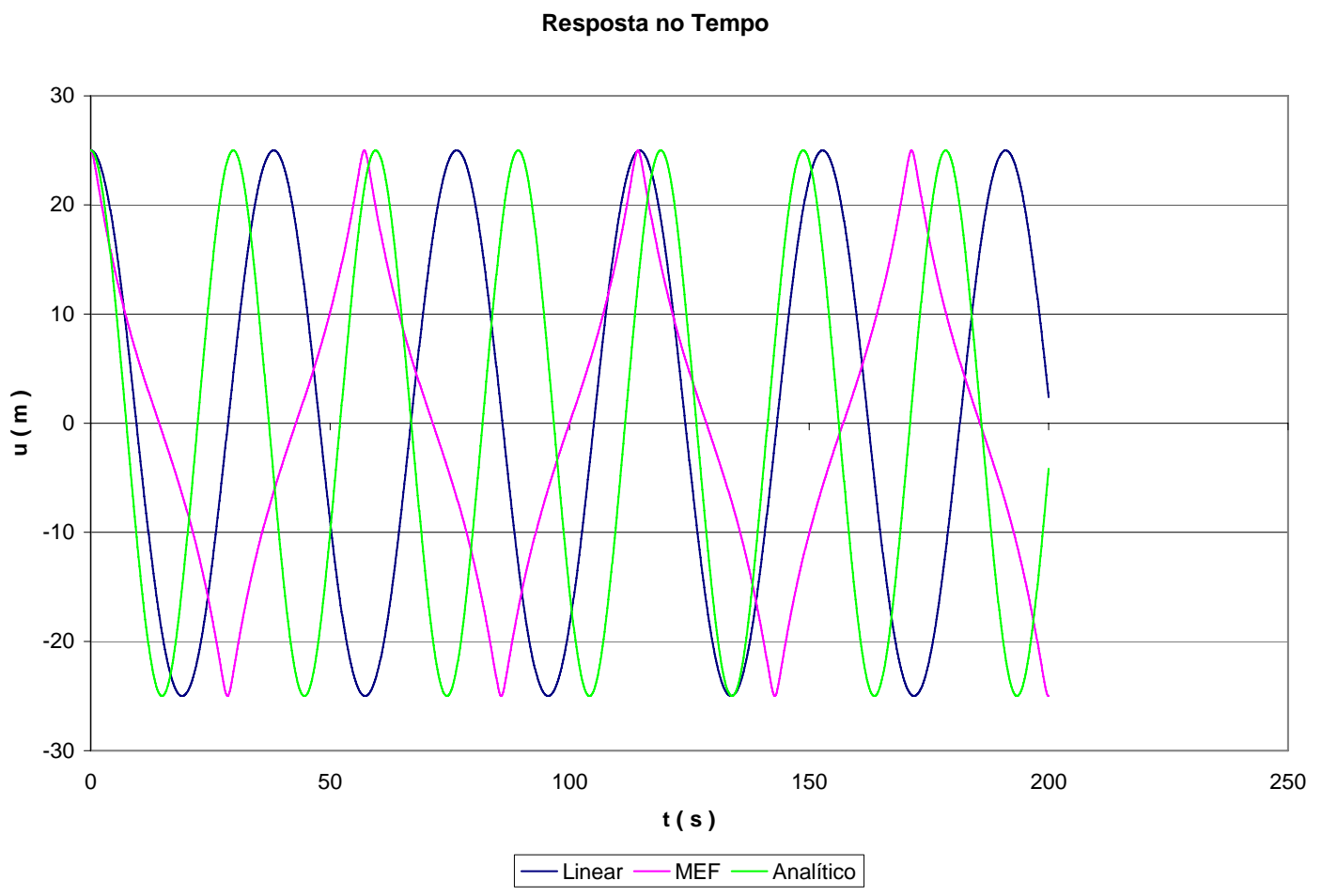

Figura 3.19 - Resposta no tempo para o primeiro modo; amplitude inicial de $25 \mathrm{~m}$. Períodos: 38,21 s (linear); 57,13 s (MEF); 29,75 s (analítico)

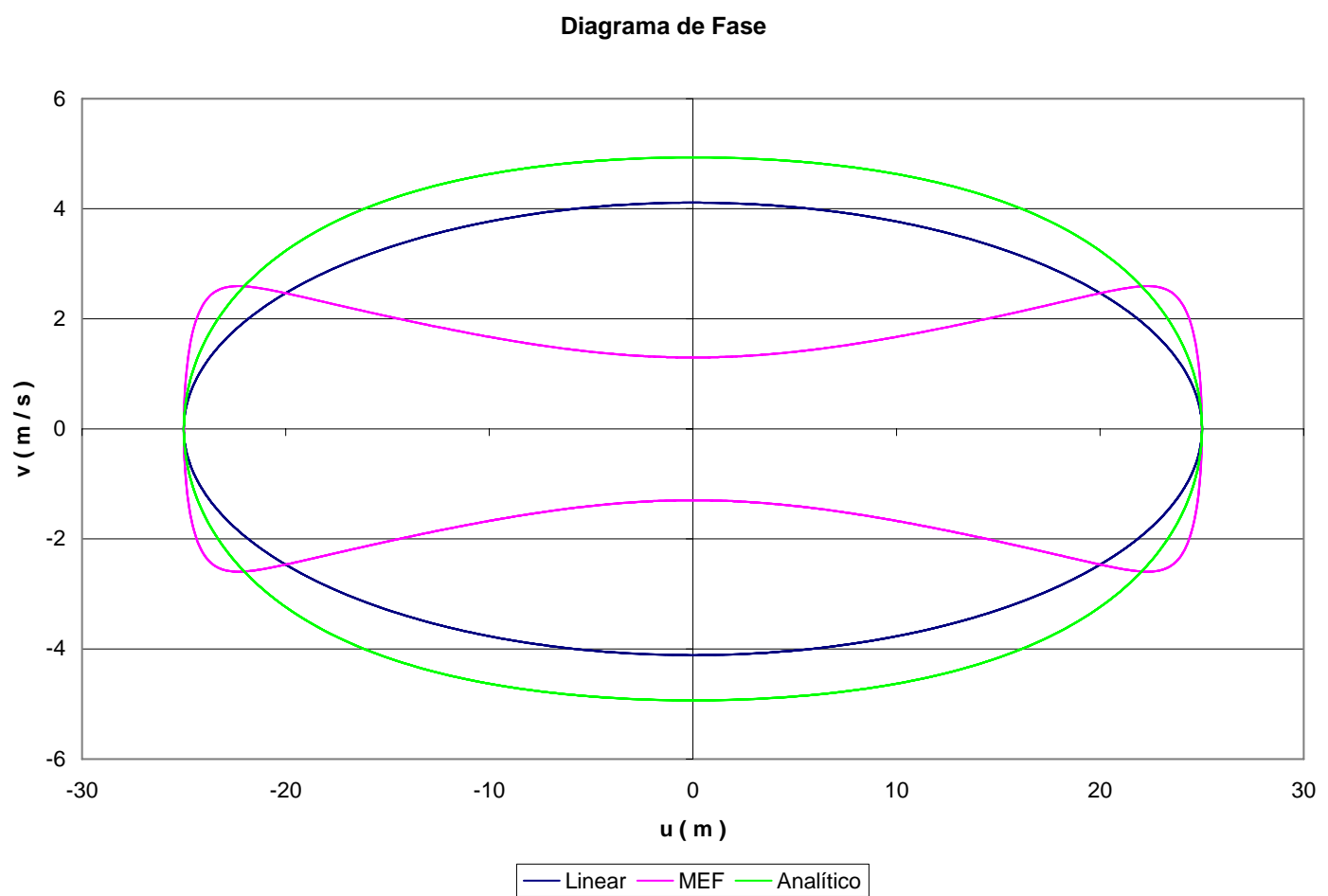

Figura 3.20 - Diagrama de fase para o primeiro modo; amplitude inicial de $25 \mathrm{~m}$. 


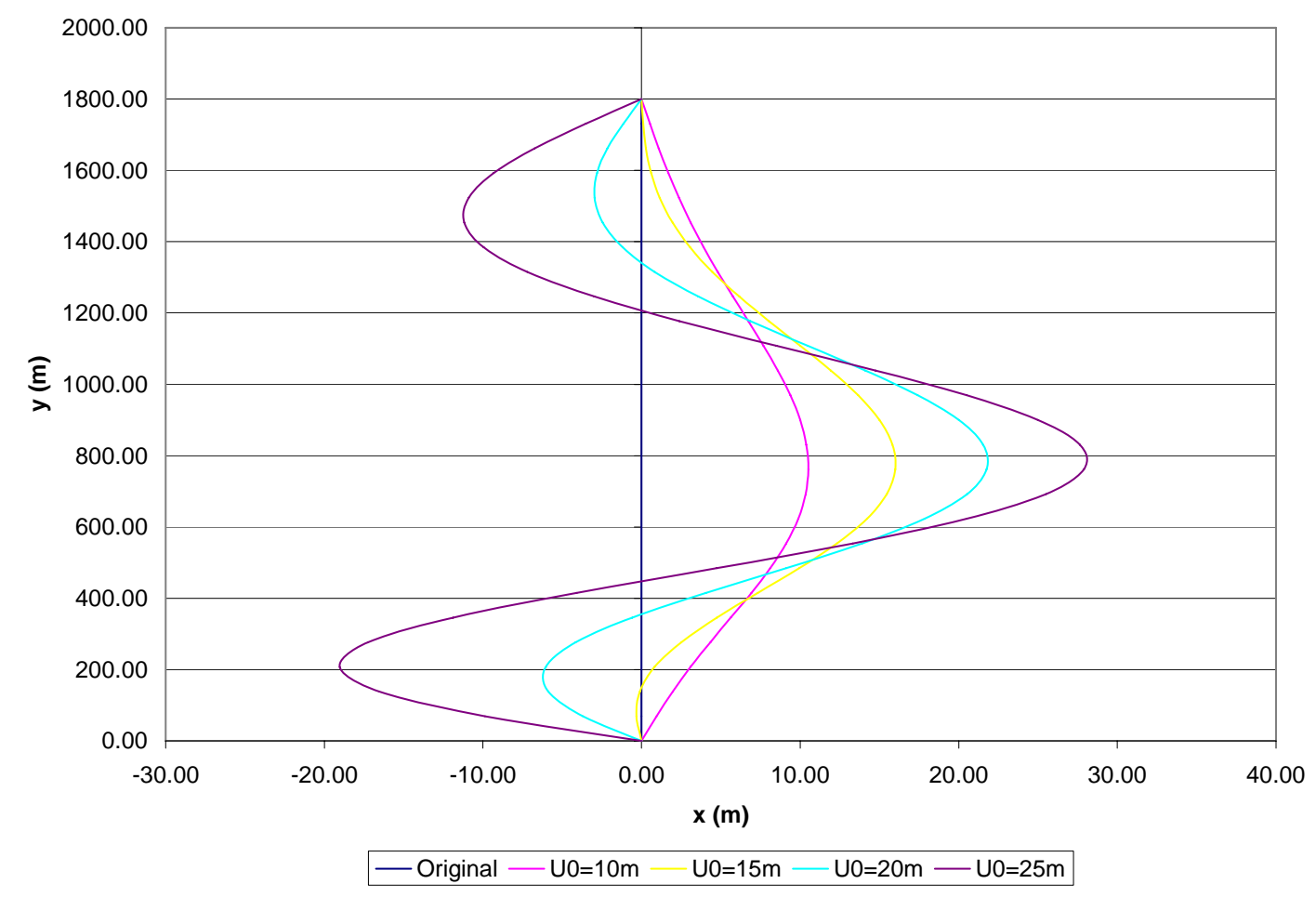

Figura 3.21 - Forma modal do primeiro modo de vibração normal não-linear para diferentes valores de amplitude inicial $U_{0}$. 


\section{Multi-modos Não-Lineares}

Um multi-modo não-linear, que acopla $n$ modos normais, é um padrão de vibrações livres não-lineares a partir de uma configuração estática de equilibro, que se desenvolve numa variedade invariante de dimensão $2 n$ contida no espaço de fase. Portanto essa variedade invariante é tangente no ponto de equilíbrio aos correspondentes auto planos do sistema linear (maiores detalhes ver Shaw et al [82] e Nayfeh [63]). Logo, uma vez que as condições iniciais pertençam à variedade invariante, a dinâmica do sistema se desenvolverá exclusivamente dentro dessa variedade.

Os multi-modos serão determinados de forma semelhante aos modos normais, utilizando-se o método das múltiplas escalas.

\section{Solução de Ordem $\varepsilon$}

Coletando os termos em $\varepsilon$, vem (ver capítulo 2):

$$
D_{0}^{2} w_{1}+\alpha w_{1}^{I V}-\beta w_{1}^{\prime \prime}=0
$$

A solução de 3.50 pode ser escrita na forma:

$$
w_{1 k}=Y_{k}\left(T_{2}\right) e^{i \omega_{k} T_{0}} \sin \frac{k \pi x}{\ell}+\text { C.C. }
$$

A partir deste ponto, considera-se um multi-modo que acopla o primeiro e o terceiro modo apenas. Estes modos foram escolhidos para compor o multi-modo devido às não-linearidades cúbicas que são características dos sistemas estruturais abordados neste texto. O primeiro e o terceiro modo serão identificados com os índices I e II, respectivamente. A resposta temporal de ordem $\varepsilon$, que tem contribuição exclusivamente desses dois modos, pode ser escrita na forma: 


$$
w_{1}=Y_{1}\left(T_{2}\right) e^{i \omega_{I} T_{0}} \sin \frac{\pi x}{\ell}+Y_{3}\left(T_{2}\right) e^{i \omega_{I I} T_{0}} \sin \frac{3 \pi x}{\ell}+\text { c.c. },
$$

onde

$$
\omega_{I I}=3 \omega_{I}
$$

\section{Solução de Ordem $\varepsilon^{2}$}

Coletando os termos em $\varepsilon^{2}$ e observando as condições de solvabilidade, vem:

$$
Y_{I}=Y_{I}\left(T_{2}\right), \quad Y_{I I}=Y_{I I}\left(T_{2}\right) .
$$

\section{Solução de Ordem $\varepsilon^{3}$}

Coletando os termos em $\varepsilon^{3}$, vem:

$$
D_{0}^{2} w_{3}+\alpha w_{3}^{I V}-\beta w_{3}^{\prime \prime}=-2 D_{0} D_{2} w_{1}+\mu w_{1}^{\prime \prime} \int_{0}^{\ell}\left(w_{1}^{\prime}\right)^{2} d x .
$$

A solução de 3.55 é escrita na forma:

$$
w_{3}=A_{3, I} \sin \frac{\pi x}{\ell}+A_{3, I I} \sin \frac{3 \pi x}{\ell} .
$$

Substituindo 3.56 em 3.55, vem: 


$$
\begin{aligned}
& \left(D_{0}^{2} A_{3, I}+\omega_{I}^{2} A_{3, I}\right) \sin \frac{\pi x}{\ell}+\left(D_{0}^{2} A_{3, I I}+\omega_{I I}^{2} A_{3, I I}\right) \sin \frac{3 \pi x}{\ell}= \\
& -2 i \omega_{I}\left(D_{2} Y_{I} e^{i \omega_{I} T_{0}}+c c\right) \sin \frac{\pi x}{\ell}-2 i \omega_{I I}\left(D_{2} Y_{I I} e^{i \omega_{I I} T_{0}}+c c\right) \sin \frac{3 \pi x}{\ell}- \\
& \frac{\pi^{4} \mu}{2 \ell^{3}}\left(Y_{I} e^{i \omega_{I} T_{0}}+c c\right)\left(Y_{I}^{2} e^{2 i \omega_{I} T_{0}}+Y_{I} \bar{Y}_{I}+9 Y_{I I}^{2} e^{2 i \omega_{I I} T_{0}}+9 Y_{I I} \bar{Y}_{I I}+c c\right) \sin \frac{\pi x}{\ell}- \\
& \frac{9 \pi^{4} \mu}{2 \ell^{3}}\left(Y_{I I} e^{i \omega_{I I} T_{0}}+c c\right)\left(Y_{I}^{2} e^{2 i \omega_{I} T_{0}}+Y_{I} \bar{Y}_{I}+9 Y_{I I}^{2} e^{2 i \omega_{I I} T_{0}}+9 Y_{I I} \bar{Y}_{I I}+c C\right) \sin \frac{3 \pi x}{\ell} .
\end{aligned}
$$

Coletando os termos em $\sin \frac{\pi x}{\ell}$ na expressão 3.57 :

$$
\begin{aligned}
& D_{0}^{2} A_{3, I}+\omega_{I}^{2} A_{3, I}=-2 i \omega_{I}\left(D_{2} Y_{I} e^{i \omega_{I} T_{0}}+c c\right)- \\
& \frac{\pi^{4} \mu}{2 \ell^{3}}\left(Y_{I} e^{i \omega_{I} T_{0}}+c c\right)\left(Y_{I}^{2} e^{2 i \omega_{I} T_{0}}+Y_{I} \bar{Y}_{I}+9 Y_{I I}^{2} e^{2 i \omega_{I I} T_{0}}+9 Y_{I I} \bar{Y}_{I I}+c C\right) .
\end{aligned}
$$

Coletando os termos em $\sin \frac{3 \pi x}{\ell}$ na expressão 3.57 :

$$
\begin{aligned}
& D_{0}^{2} A_{3, I I}+\omega_{I I}^{2} A_{3, I I}=-2 i \omega_{I I}\left(D_{2} Y_{I I} e^{i \omega_{I I} T_{0}}+c C\right)- \\
& \frac{9 \pi^{4} \mu}{2 \ell^{3}}\left(Y_{I I} e^{i \omega_{I I} T_{0}}+c C\right)\left(Y_{I}^{2} e^{2 i \omega_{I} T_{0}}+Y_{I} \bar{Y}_{I}+9 Y_{I I}^{2} e^{2 i \omega_{I I} T_{0}}+9 Y_{I I} \bar{Y}_{I I}+C C\right)
\end{aligned}
$$

A eliminação dos termos seculares em 3.58 e 3.59 leva a:

$$
\begin{aligned}
& -2 i \omega_{I} D_{2} Y_{I}-3 \Lambda_{1} Y_{I}^{2} \bar{Y}_{I}-18 \Lambda_{1} Y_{I} Y_{I I} \bar{Y}_{I I}=0, \\
& -2 i \omega_{I I} D_{2} Y_{I I}-18 \Lambda_{1} Y_{I} \bar{Y}_{I} Y_{I I}-243 \Lambda_{1} Y_{I I}^{2} \bar{Y}_{I I}=0,
\end{aligned}
$$

Separando as partes reais e imaginárias, as seguintes equações diferenciais são integradas: 


$$
\begin{aligned}
& D_{2} a_{I}^{\prime}=0 \Rightarrow a_{I}=\text { const }, \quad D_{2} a_{I I}^{\prime}=0 \Rightarrow a_{I I}=\text { const }, \\
& \omega_{I} a_{I} D_{2} \theta_{I}-\frac{3 \Lambda_{1}}{8} a_{I}^{3}-\frac{18 \Lambda_{1}}{8} a_{I} a_{I I}^{2}=0 \Rightarrow \theta_{I}=\theta_{I, 0}+\frac{\Lambda_{1}}{8 \omega_{I}}\left(3 a_{I}^{2}+18 a_{I I}^{2}\right) \Gamma_{2} \\
& \omega_{I I} a_{I I} D_{2} \theta_{I I}-\frac{18 \Lambda_{1}}{8} a_{I}^{2} a_{I I}-\frac{243 \Lambda_{1}}{8} a_{I I}^{3}=0 \Rightarrow \theta_{I I}=\theta_{I I, 0}+\frac{\Lambda_{1}}{8 \omega_{I I}}\left(18 a_{I}^{2}+243 a_{I I}^{2}\right) \Gamma_{2} .
\end{aligned}
$$

Após a eliminação dos termos seculares, as soluções podem ser escritas como:

$$
\begin{aligned}
& A_{3, I}=\frac{\Lambda_{1}}{8 \omega_{I}^{2}} Y_{I}^{3} e^{3 i \omega_{I} T_{0}}+\frac{9 \Lambda_{1}}{24 \omega_{I}^{2}} \bar{Y}_{I} Y_{I I}^{2} e^{i\left(2 \omega_{I I}-\omega_{I}\right) T_{0}}+\frac{9 \Lambda_{1}}{48 \omega_{I}^{2}} Y_{I} Y_{I I}^{2} e^{i\left(2 \omega_{I I}+\omega_{I}\right) T_{0}}+c C, \\
& A_{3, I I}=-\frac{9 \Lambda_{1}}{8 \omega_{I}^{2}} \bar{Y}_{I}^{2} Y_{I I} e^{i\left(\omega_{I I}-2 \omega_{I}\right) T_{0}}+\frac{9 \Lambda_{1}}{16 \omega_{I}^{2}} Y_{I}^{2} Y_{I I} e^{i\left(\omega_{I I}+2 \omega_{I}\right) T_{0}}+\frac{81 \Lambda_{1}}{72 \omega_{I}^{2}} Y_{I I}^{3} e^{3 i \omega_{I I} T_{0}}+C C .
\end{aligned}
$$

As expressões 3.63 e 3.64 definem o multi-modo que acopla o primeiro e terceiro modos. 


$$
\begin{aligned}
& w_{I, I I}(x, t)=\left(a_{I}\right)\left[\cos \left(\Omega_{I} t+\theta_{I, 0}\right)+\frac{\Lambda_{1}}{32 \omega_{I}^{2}}\left(z_{I}\right)^{2} \cos 3\left(\Omega_{I} t+\theta_{I, 0}\right)\right] \sin \frac{\pi x}{\ell} \\
& +\left({z a_{I}}_{I}\right)\left(2 a_{I I}\right)^{2}\left\{\begin{array}{l}
\frac{9 \Lambda_{1}}{96 \omega_{I}^{2}} \cos \left[\left(2 \Omega_{I I}-\Omega_{I}\right) t+2 \theta_{I I, 0}-\theta_{I, 0}\right]+ \\
+\frac{9 \Lambda_{1}}{192 \omega_{I}^{2}} \cos \left[\left(2 \Omega_{I I}+\Omega_{I}\right) t+2 \theta_{I I, 0}+\theta_{I, 0}\right]
\end{array}\right\} \sin \frac{\pi x}{\ell} \\
& +\left(ฉ_{I I}\right)\left[\cos \left(\Omega_{I I} t+\theta_{I I, 0}\right)+\frac{81 \Lambda_{1}}{288 \omega_{I}^{2}}\left(æ a_{I I}\right)^{2} \cos 3\left(\Omega_{I I} t+\theta_{I I, 0}\right)\right] \sin \frac{3 \pi x}{\ell} \\
& +\left(a_{I I}\right)\left({\left.ฉ a_{I}\right)^{2}}^{2}\left\{\begin{array}{l}
\frac{9 \Lambda_{1}}{64 \omega_{I}^{2}} \cos \left[\left(\Omega_{I I}+2 \Omega_{I}\right) t+\theta_{I I, 0}+2 \theta_{I, 0}\right]+ \\
-\frac{9 \Lambda_{1}}{32 \omega_{I}^{2}} \cos \left[\left(\Omega_{I I}-2 \Omega_{I}\right) t+\theta_{I I, 0}-2 \theta_{I, 0}\right]
\end{array}\right\} \sin \frac{3 \pi x}{\ell},\right. \\
& u_{I, I I}(x, t)=-\frac{\pi}{16 \ell}\left(z a_{I}\right)^{2}\left[1+\cos 2\left(\Omega_{I} t+\theta_{I, 0}\right)\right] \sin \frac{2 \pi x}{\ell}+ \\
& -\frac{3 \pi}{16 \ell}\left(z a_{I I}\right)^{2}\left[1+\cos 2\left(\Omega_{I I} t+\theta_{I I, 0}\right)\right] \sin \frac{6 \pi x}{\ell}+ \\
& -\frac{3 \pi}{8 \ell}\left(a a_{I}\right)\left(2 a_{I I}\right) \cos \left(\Omega_{I} t+\theta_{I, 0}\right) \cos \left(\Omega_{I I} t+\theta_{I I, 0}\right)\left(2 \sin \frac{2 \pi x}{\ell}+\sin \frac{4 \pi x}{\ell}\right)+O\left(\varepsilon^{4}\right) \\
& \Omega_{I}=\omega_{I}+\frac{\Lambda_{1}}{8 \omega_{I}}\left[3\left(z a_{I}\right)^{2}+18\left(z a_{I I}\right)^{2}\right] ; \Omega_{I I}=\omega_{I I}+\frac{\Lambda_{1}}{8 \omega_{I I}}\left[18\left(z a_{I}\right)^{2}+243\left(z a_{I I}\right)^{2}\right] .
\end{aligned}
$$

Um programa de computador para cálculo automático dos multi-modos foi desenvolvido por Baracho Neto [6]. Este programa utiliza o M.E.F. para a discretização do sistema estrutural e o método das múltiplas escalas para determinação dos multi-modos.

A seguir será apresentado o multi-modo 1:3 (leia-se o multi-modo que acopla o primeiro e o terceiro modos) para o riser reto com peso próprio calculado analiticamente e numericamente (via M.E.F.). 

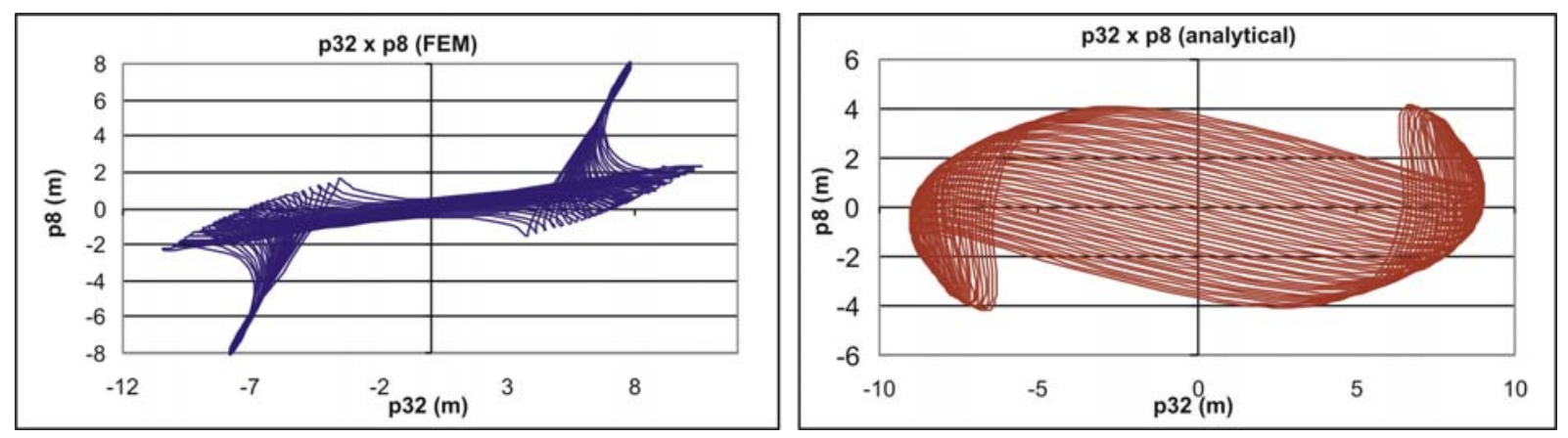

Figura $3.22-$ Diagramas de fase $p_{32}=w(0.42 \ell) \times p_{8}=w(0.12 \ell)$ para o multimodo não-linear obtido analiticamente (direita) e via MEF (esquerda).

A figura 3.22 mostra as trajetórias de fase do multi-modo 1:3 projetadas no plano $p_{32} \times p_{8}$. Escolheu-se os deslocamentos $p_{32}$ e $p_{8}$ como variáveis modais, pois o primeiro é o grau de liberdade (deslocamento transversal próximo do meio do comprimento - $0.42 \ell$ ) com maior amplitude para o primeiro modo, e o segundo é o grau de liberdade (deslocamento transversal de um ponto localizado a uma distância $0.12 \ell$ da superfície) com maior amplitude para o terceiro modo. A trajetória à esquerda foi obtida via M.E.F. e a da direita foi obtida analiticamente. As duas trajetórias de fase são qualitativamente similares, vale lembrar que ambas as abordagens se tratam de aproximações e que, portanto, ambas são corretas dentro de suas hipóteses de aplicação.

A figura 3.23 apresenta os diagramas de fase $p_{8} \times \dot{p}_{8}$ e $p_{32} \times \dot{p}_{32}$. Observa-se um movimento menor próximo do deslocamento inicial seguido por um movimento maior que leva o sistema a um novo atrator simétrico ao primeiro. A interação entre os dois modos acoplados (primeiro e terceiro) é capturada pelo multi-modo. Portanto, acredita-se que multi-modos sejam capazes de representar o principal comportamento global dos sistemas estruturais com um número reduzido de graus de liberdade. 

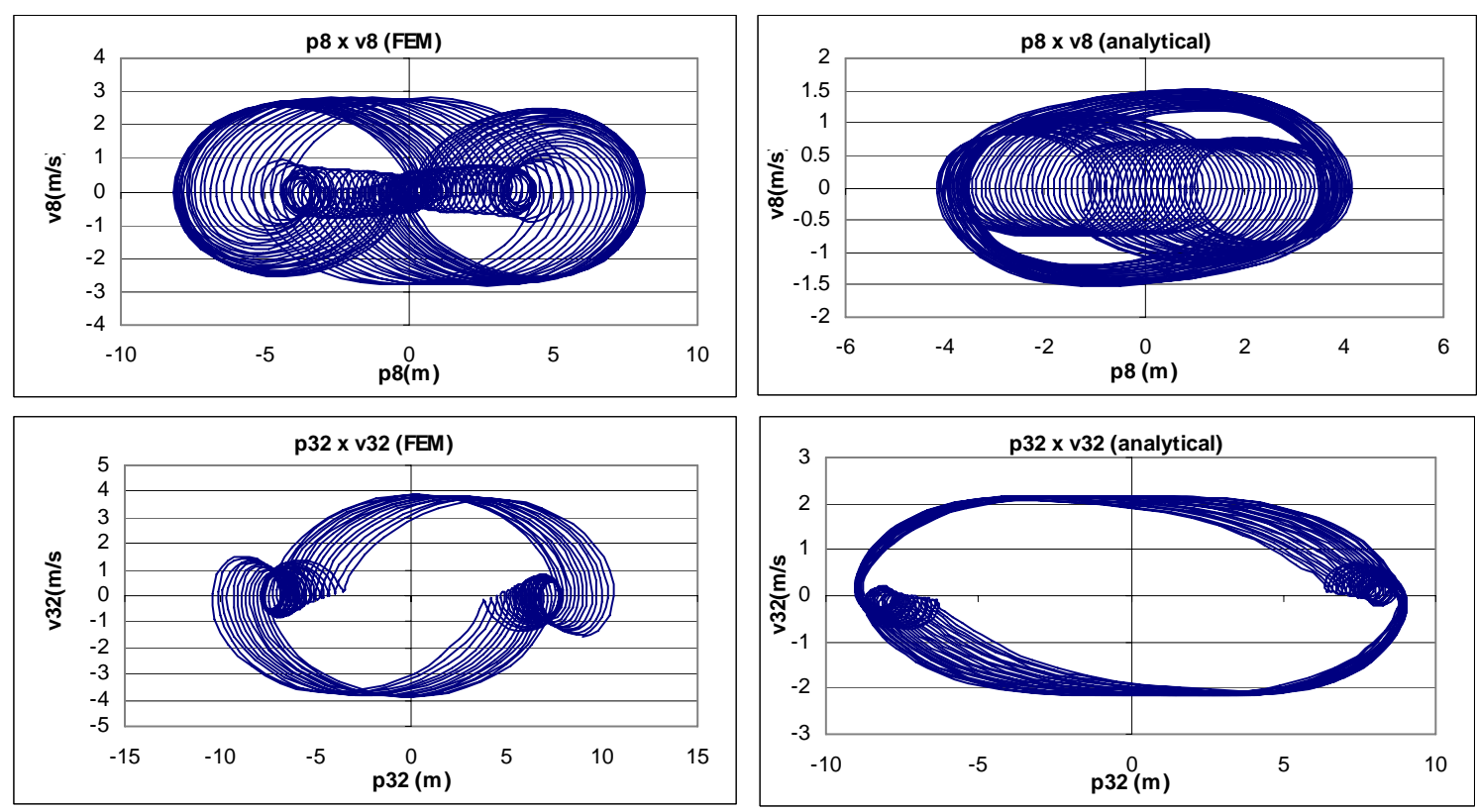

Figura 3.23 - Diagramas de fase $p_{8} \times \dot{p}_{8}=v_{8}$ and $p_{32} \times \dot{p}_{32}=v_{32}$ para as interações multi-modais obtidas analiticamente (direita) e via MEF (esquerda).

A figura 3.24 apresenta instantâneos do movimento do multi-modo e os modos acoplados desenvolvendo-se no tempo. O intervalo de tempo apresentado é de meio período. No instante $t=0$ nota-se a participação significativa do terceiro modo influenciando a forma da resposta composta. Contrariamente, a participação do terceiro modo um doze avos de período à frente $t=3.1 \mathrm{~s}$ é quase nulo uma vez que a freqüência do terceiro modo linear é três a do primeiro modo linear. Com o sexto do período $t=6 \mathrm{~s}$ o terceiro modo alcança outro máximo enquanto o primeiro modo caminha para configuração de equilíbrio, neste instante o terceiro modo tem sua maior influência na resposta. Com o quarto de período $t=9 \mathrm{~s}$, o primeiro e o terceiro modos quase se anulam ao mesmo tempo. De forma análoga aos instantes anteriores, o primeiro e o terceiro modos interagem para compor a resposta total alternando suas participações nos instantes 12.2 e 15.3s. O meio período vale $18.4 \mathrm{~s}$. O último quadro foi omitido por ser simétrico ao primeiro. 
Modal Response $t=0 \mathrm{~s}$

(a)

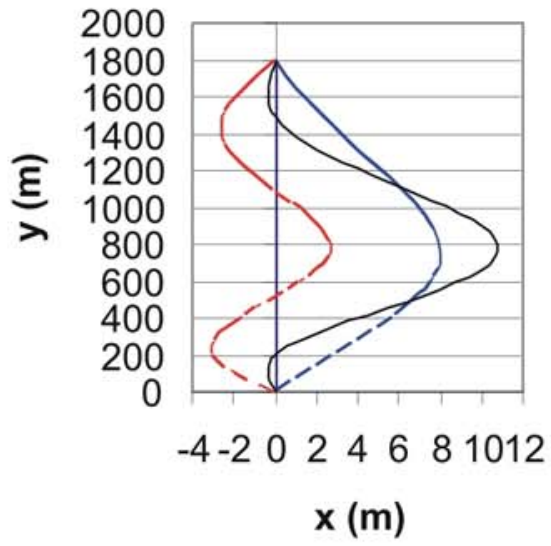

Modal Response $t=6.1 \mathrm{~s}$

(c)

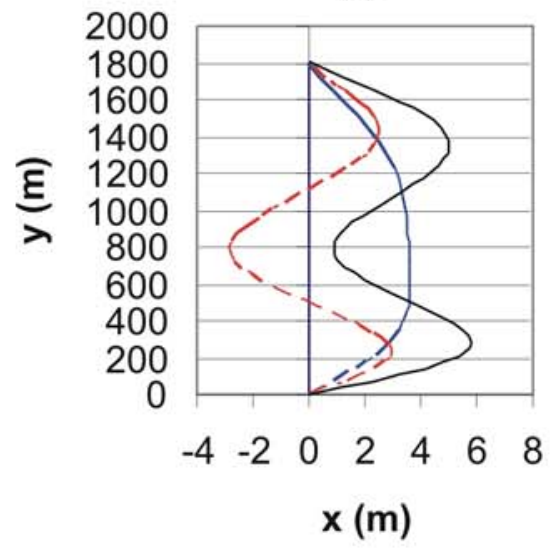

Modal Response $t=12.2 \mathrm{~s}$
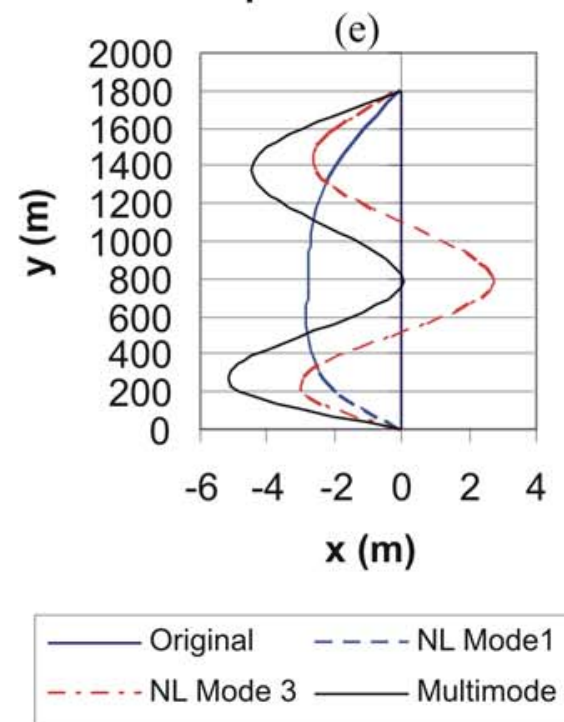

Modal Response $t=3.1 \mathrm{~s}$

(b)

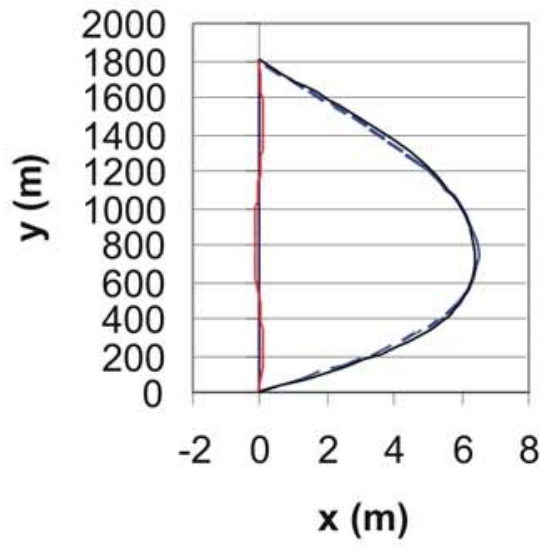

Modal Response $t=9.2 \mathrm{~s}$

(d)

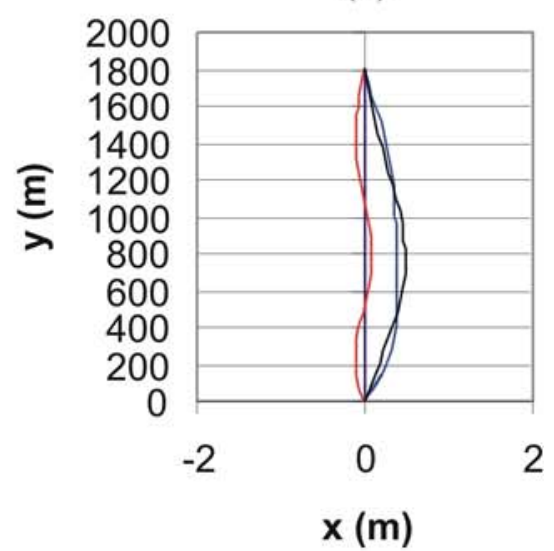

Modal Response $t=15.3 \mathrm{~s}$

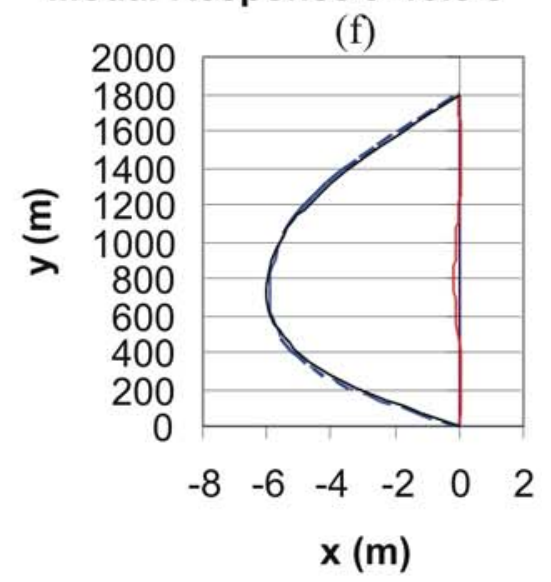

$\begin{array}{ll}- \text { Original } & ---- \text { NL Mode } 1 \\ -\cdots \text { NL Mode } 3 \longrightarrow \text { Multimode }\end{array}$

Figura 3.24 - Fotografias do multi-modo (acopla primeiro e terceiro modos lineares). 


\subsection{Correnteza}

Nesta seção aborda-se o mesmo riser da seção anterior acrescendo-se uma correnteza perpendicular ao eixo longitudinal $(x)$ com perfil de velocidade constante ao longo de todo o comprimento da estrutura. As propriedades físicas do riser e da corrente encontram-se na tabela 3.3.

\begin{tabular}{|l|c|}
\hline Módulo de Young & $E=2,1 \times 10^{11} \mathrm{~N} / \mathrm{m}^{2}$ \\
\hline Comprimento & $\ell=1800 \mathrm{~m}$ \\
\hline Área da Seção Transversal & $A=1,1021 \times 10^{-2} \mathrm{~m}^{2}$ \\
\hline Momento de Inércia & $I=4,72143 \times 10^{-5} \mathrm{~m}^{4}$ \\
\hline Força Normal Inicial (no topo) & $N_{0}=2 \times 10^{6} \mathrm{~N}$ \\
\hline $\begin{array}{l}\text { Massa por unidade de comprimento (água interna }+ \\
\text { massa adicionada) }\end{array}$ & $m=141,24 \mathrm{~kg} / \mathrm{m}$ \\
\hline Peso submerso do riser por unidade de comprimento & $p=727 \mathrm{~N} / \mathrm{m}$ \\
\hline Velocidade da corrente & $U=0,5 \mathrm{~m} / \mathrm{s}$ \\
\hline Coeficiente de arrasto & $C_{D}=1,2$ \\
\hline Peso específico da água do mar & $\rho_{a}=1025 \mathrm{~kg} / \mathrm{m}^{3}$ \\
\hline Diâmetro externo & $D=0,2032 \mathrm{~m}$ \\
\hline
\end{tabular}

Tabela 3.3 - Propriedades físicas do riser e da corrente.

A figura 3.25 mostra uma representação esquemática do sistema estrutural. O carregamento distribuído oriundo da aplicação da corrente é calculado segundo a clássica equação de Morrison, ver [69]:

$$
f_{c}=\frac{1}{2} C_{D} \rho_{a} D U|U|
$$

onde $C_{D}$ é o coeficiente de arrasto, $\rho_{a}$ é o peso específico da água, $D$ é o diâmetro do riser e $U$ é a velocidade da corrente. De forma geral, a consideração da corrente é feita como um carregamento estático, ou seja, nenhum efeito dinâmico, como VIV, é abordado nesta seção. O carregamento distribuído devido à correnteza aumentou as forças normais iniciais nos elementos. Sendo assim, as freqüências tendem a ser ligeiramente superiores às freqüências do exemplo sem corrente. 
Os modos não-lineares são determinados com o auxílio do programa de cálculo modonlr. A discretização do modelo de elementos finitos é a mesma da figura 3.2 .

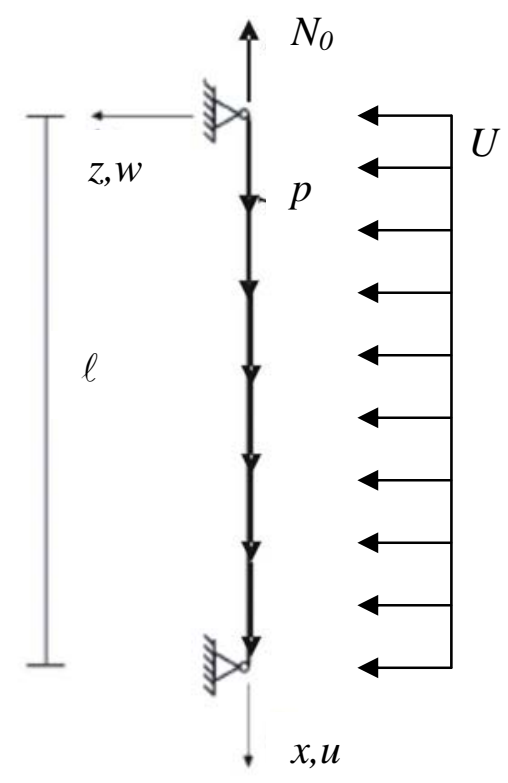

Figura 3.25 - Riser com corrente constante de velocidade $U$.

Utilizando-se o M.E.F. e a abordagem por variedades invariantes, encontra-se a seguinte equação do oscilador modal para o primeiro modo:

$$
\begin{aligned}
& \ddot{U}+2,71 \times 10^{-2} U-2,74 \times 10^{-14} V-6,70 \times 10^{-16} U^{2}-8,08 \times 10^{-17} U V+2,71 \times 10^{-14} V^{2} \\
& +2,49 \times 10^{-4} U^{3}-9,59 \times 10^{-14} U^{2} V-2,23 \times 10^{-2} U V^{2}+1,19 \times 10^{-12} V^{3}=0
\end{aligned} .
$$

Estuda-se, como já mencionado anteriormente, apenas o primeiro modo nãolinear deste exemplo com corrente.

Na equação 3.66, os coeficientes de $V, U^{2}, U V, V^{2}, U^{2} V$ e $V^{3}$ são desprezíveis. De maneira simplificada, a equação 3.66 pode ser reescrita na forma:

$$
\ddot{U}+2,71 \times 10^{-2} U+2,49 \times 10^{-4} U^{3}-2,23 \times 10^{-2} U V^{2}=0
$$


Nota-se que o módulo do coeficiente de $U V^{2}$ para o exemplo com apenas o peso próprio é $1.79 \%$ maior que o módulo do mesmo coeficiente para o exemplo com corrente. Não obstante, o coeficiente (no caso com apenas peso próprio) de $U$ é $0.37 \%$ menor que o mesmo coeficiente no caso com corrente. No entanto, ambas as variações apontam para a mesma tendência de enrijecimento. Este último era previsível uma vez que a rigidez do sistema estrutural é dependente da intensidade da força normal de tração. Com a aplicação da corrente, a força normal de tração sofre um aumento ao longo de todo o comprimento do riser. O aumento de tração leva ao aumento da rigidez e ao conseqüente aumento da freqüência que se observa na variação dos coeficientes.

É verdade que o termo em $U^{3}$ é $1.20 \%$ maior para o caso com apenas peso próprio. Mas, para os valores de amplitude inicial do exemplo nesta seção, a velocidade modal se torna significativa e a influência do termo em $U V^{2}$ é sentida com mais força e, portanto, o que se observa é um leve aumento da freqüência do sistema estrutural.

As figuras 3.26 e 3.27 apresentam os resultados para uma amplitude inicial de $10 \mathrm{~m}$. As figuras 3.28 e 3.29 mostram os resultados para uma amplitude de $15 \mathrm{~m}$. No primeiro conjunto já é possível notar o efeito de "softening" devido às influências não -lineares, mas essa tendência fica realmente clara para o caso com amplitude de $15 \mathrm{~m}$. Neste caso, o "estrangulamento" do diagrama de fase fica nítido e a freqüência do sistema cai significativamente.

As figuras 3.30 e 3.31 mostram resultados para amplitude de $20 \mathrm{~m}$ e as figuras 3.32 e 3.33 para amplitude $25 \mathrm{~m}$. Para estes casos, além do "estrangulamento" do diagrama de fase, fica evidente também a mudança do comportamento da resposta no tempo, que se distancia do padrão senoidal da solução linear. 


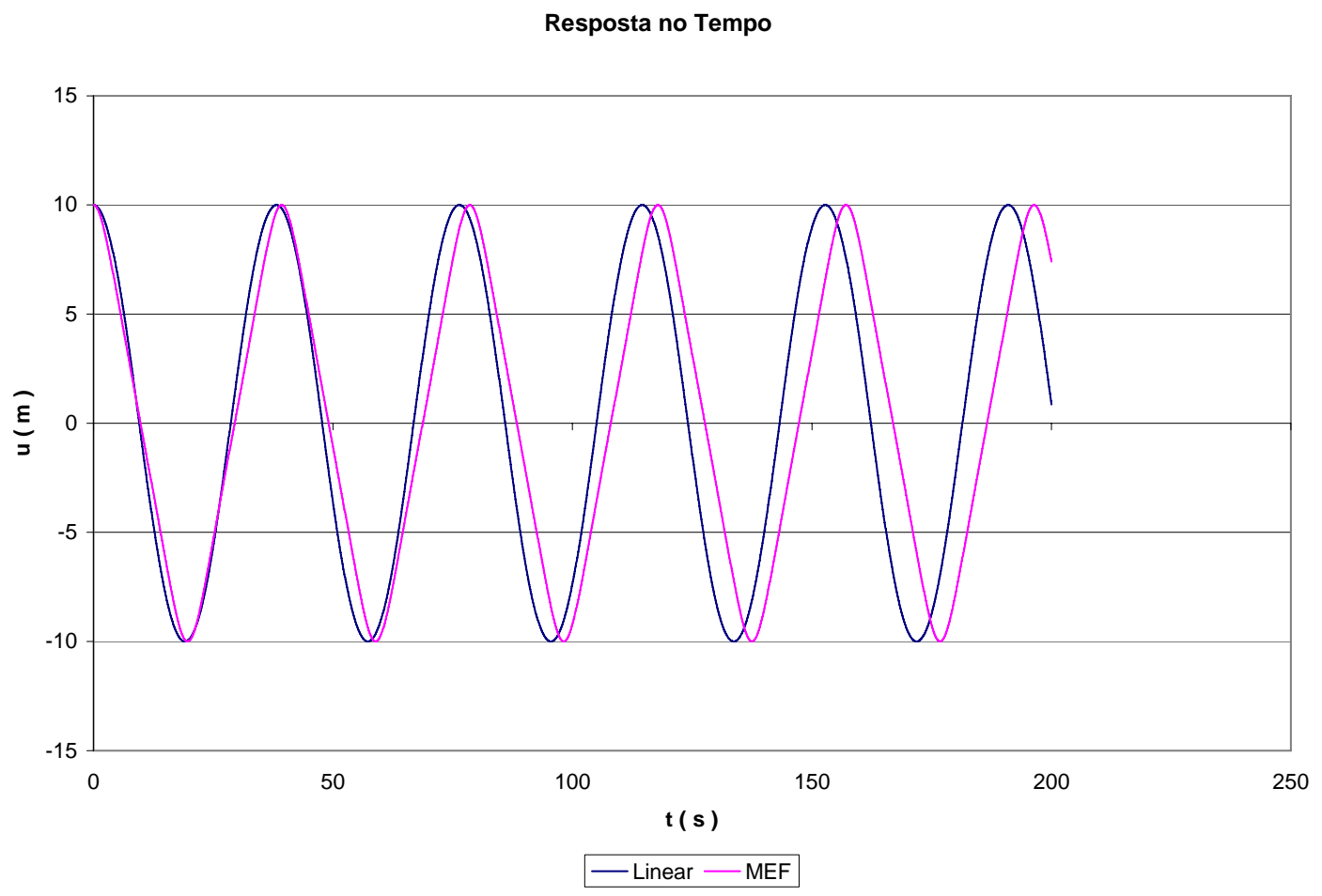

Figura 3.26 - Resposta no tempo para o primeiro modo; amplitude inicial de $10 \mathrm{~m}$. Períodos: 38,19 s (linear); 39,28 s (MEF).

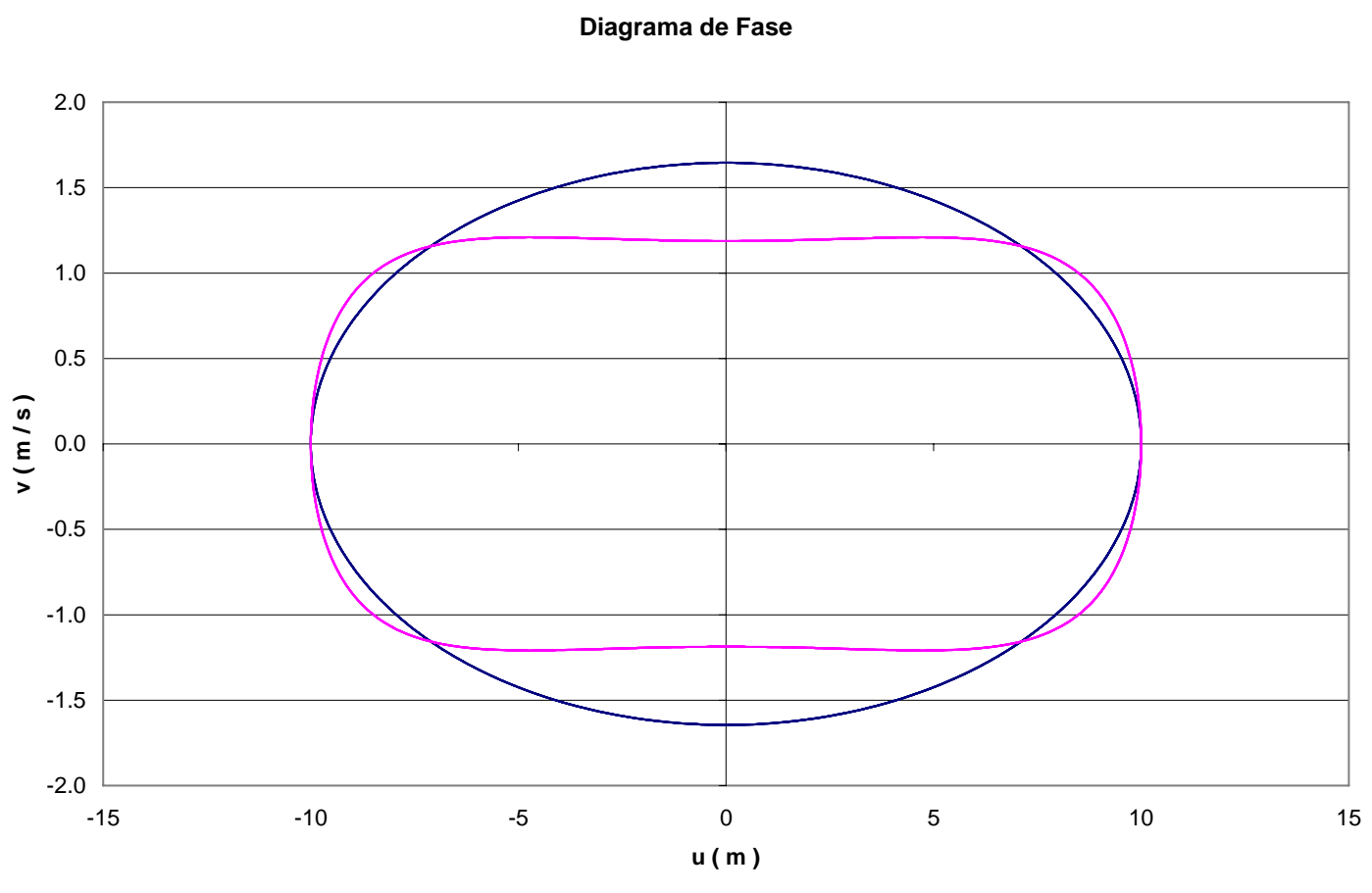

- Linear - MEF

Figura 3.27 - Diagrama de fase para o primeiro modo; amplitude inicial de $10 \mathrm{~m}$. 


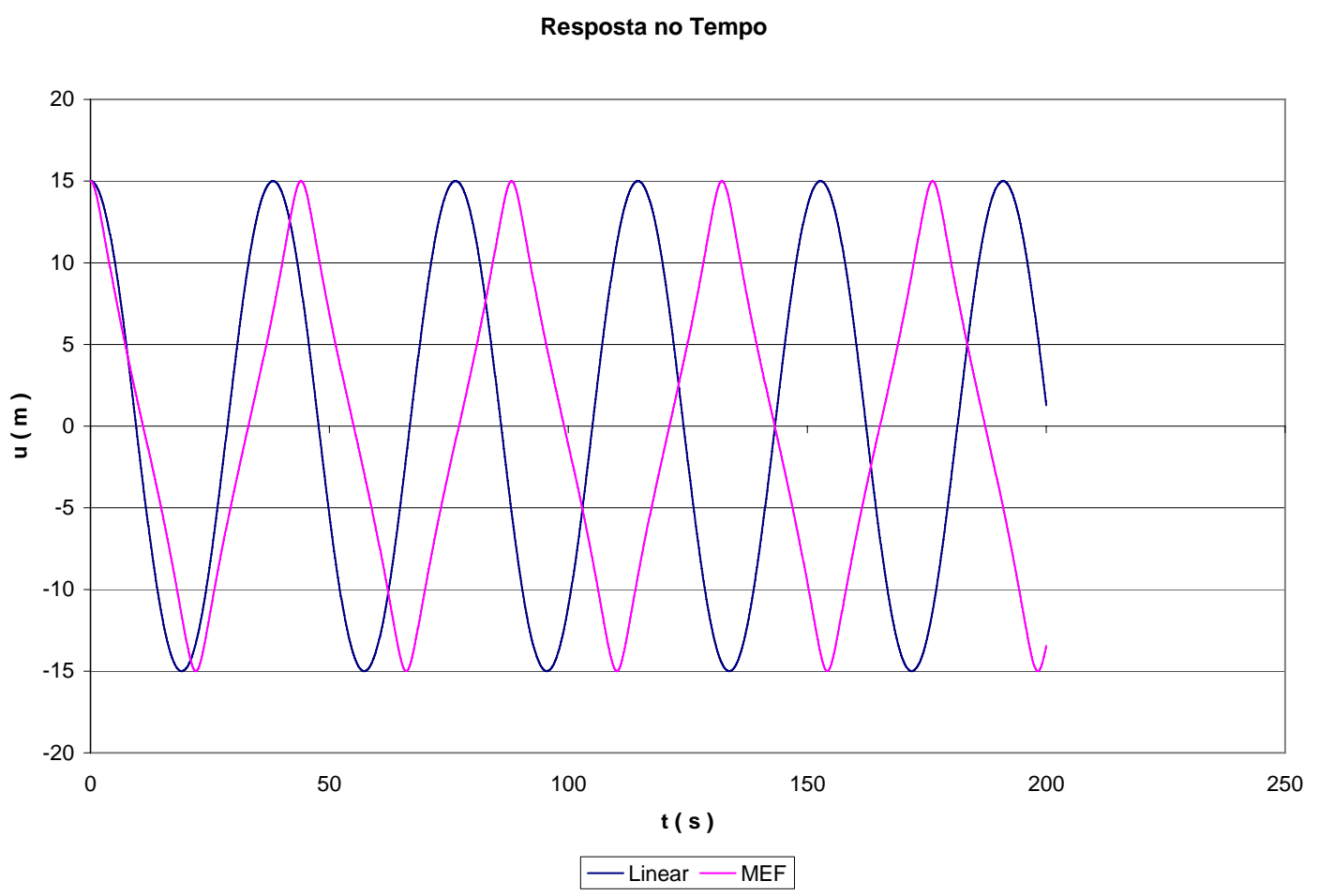

Figura 3.28 - Resposta no tempo para o primeiro modo; amplitude inicial de $15 \mathrm{~m}$. Períodos: 38,19 s (linear); $44.06 \mathrm{~s}$ (MEF).

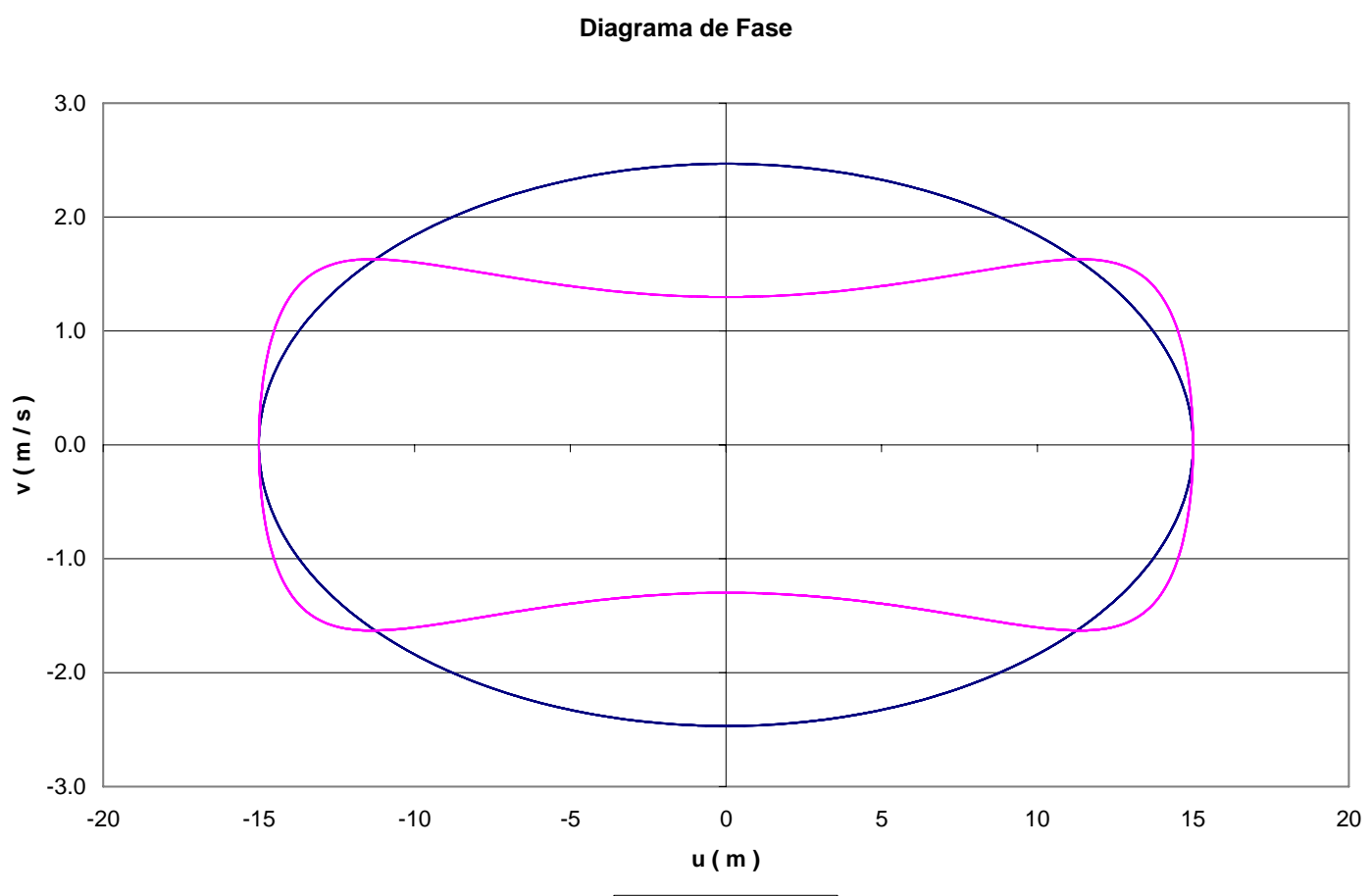

Figura 3.29 - Diagrama de fase para o primeiro modo; amplitude inicial de $15 \mathrm{~m}$. 


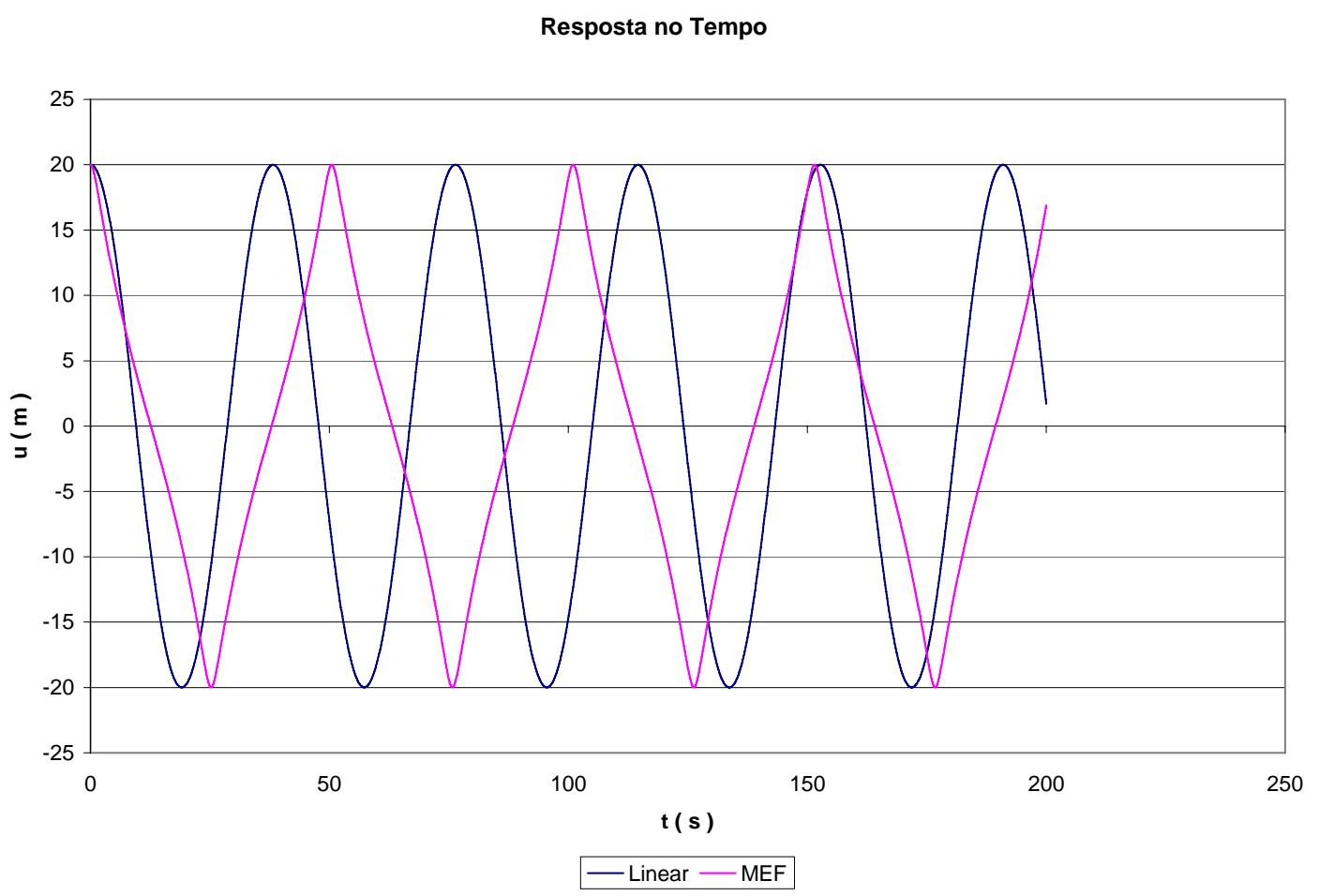

Figura 3.30 - Resposta no tempo para o primeiro modo; amplitude inicial de $20 \mathrm{~m}$. Períodos: 38,19 s (linear); 50,50 s (MEF).

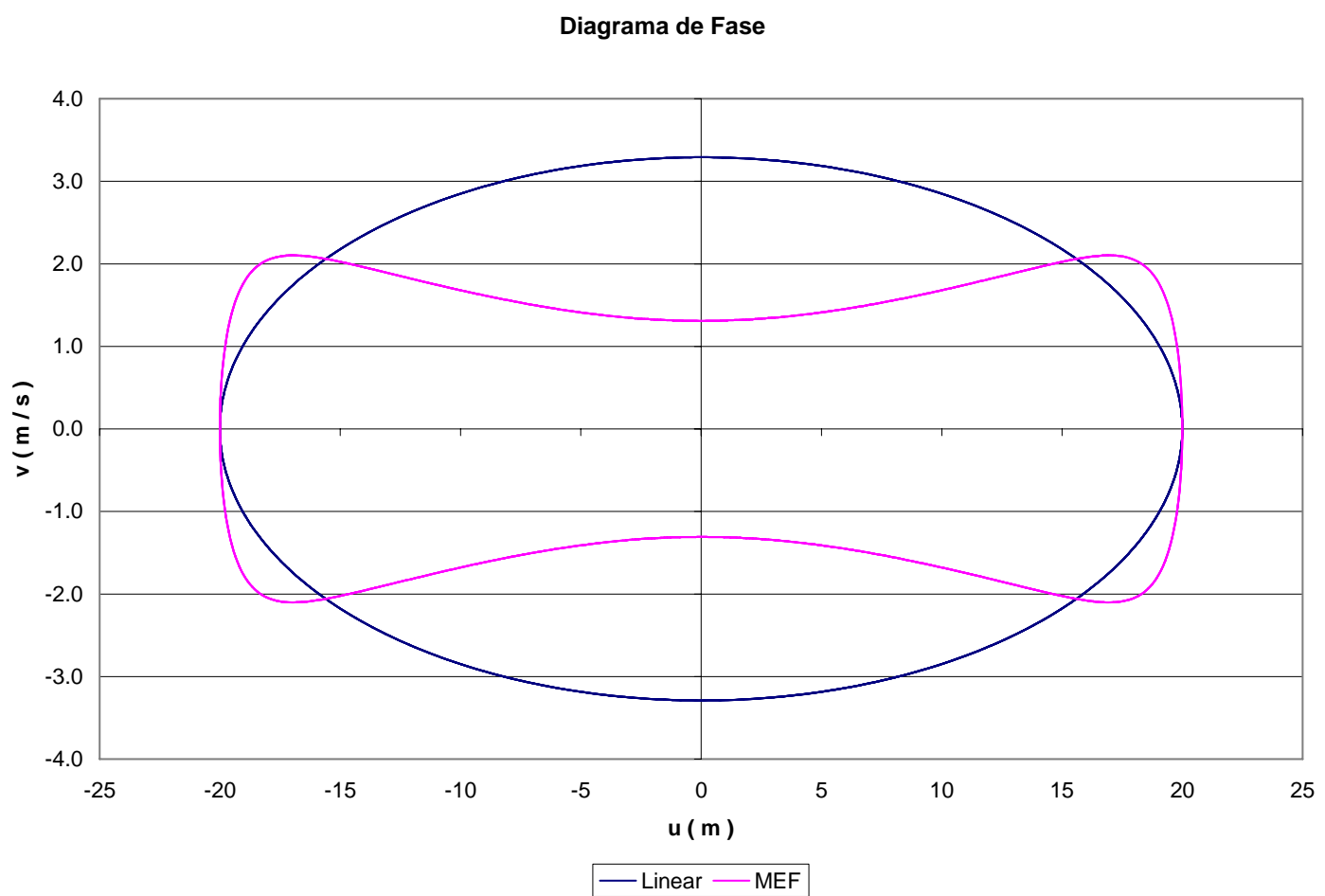

Figura 3.31 - Diagrama de fase para o primeiro modo; amplitude inicial de $20 \mathrm{~m}$. 


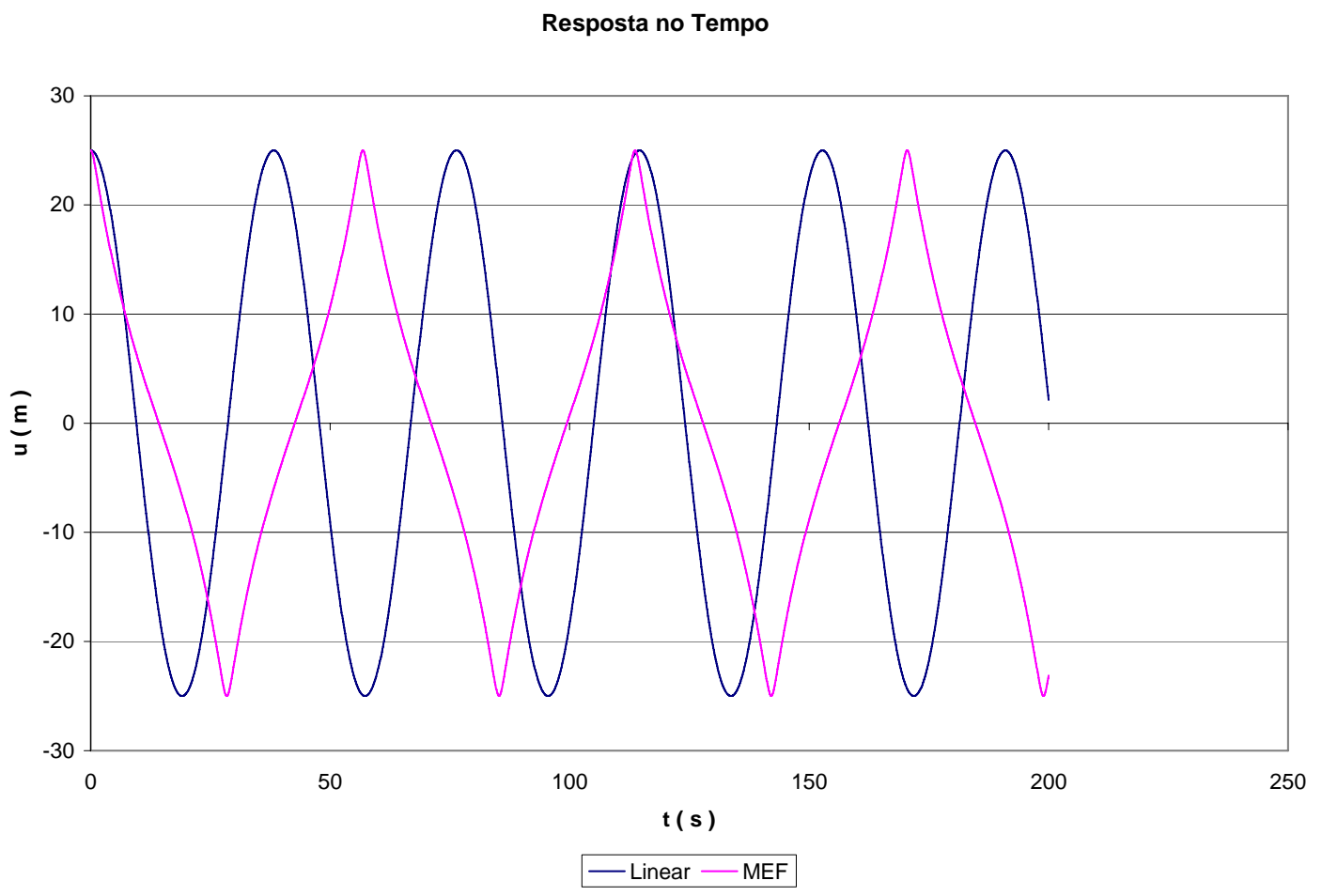

Figura 3.32 - Resposta no tempo para o primeiro modo; amplitude inicial de $25 \mathrm{~m}$. Períodos: 38,19 s (linear); 56,84 s (MEF).

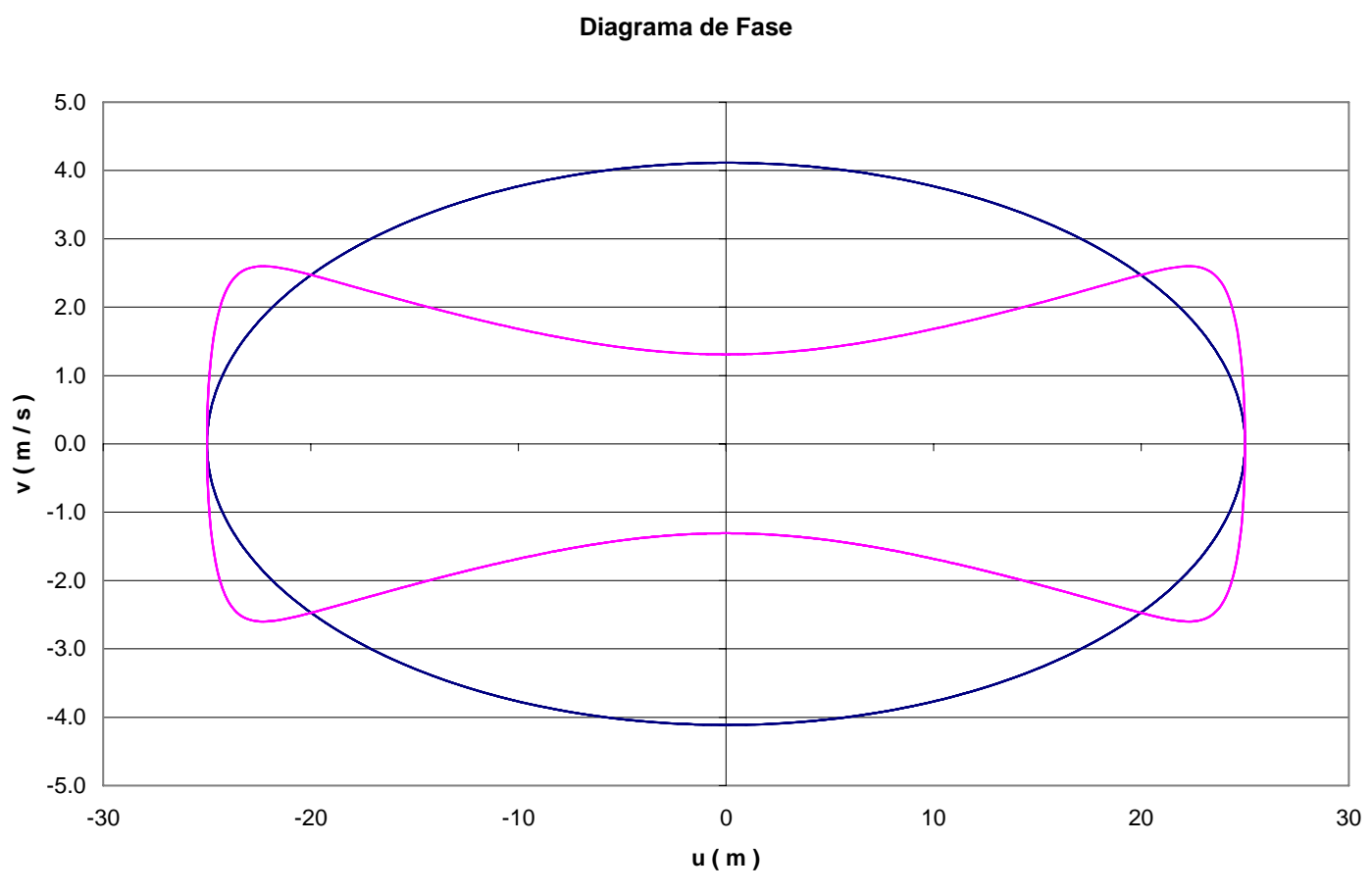

- Linear - MEF

Figura 3.33 - Diagrama de fase para o primeiro modo; amplitude inicial de $25 \mathrm{~m}$. 


\subsubsection{Vibração Induzida por Vórtices - Riser Reto}

Um corpo rombudo é definido como sendo aquele que, no caso de ser imerso em uma corrente de fluido, gera separação do escoamento e esta ocorre em uma porção considerável de sua superfície, ver [56]. Tais corpos, em nossas aplicações, são os risers. Esta separação, em um corpo com seção transversal bidimensional, faz com que haja a formação de duas camadas cisalhantes livres na região imediatamente posterior ao corpo. Estas duas camadas possuem vorticidade de sinal oposto. A interação destas duas camadas cisalhantes de fluido representam a razão principal da formação e desprendimento de vórtices atrás do corpo. O número de Strouhal $S_{t}=\frac{f D}{U}$ relaciona a freqüência de desprendimento de vórtices $f$, o diâmetro da seção transversal $D$ e a velocidade da corrente $U$. A vibração induzida pelo desprendimento de vórtices é perpendicular à direção de escoamento do fluido. Sendo assim, para todos os exemplos de VIV neste texto, admite-se que a direção de escoamento do fluido seja perpendicular ao plano que contém o movimento. A fileira dupla de vórtices, idealizada por Von Kármán, que se forma atrás de um corpo rombudo bidimensional pode ser idealizada, para um fluido invíscido, de forma que cada vórtice é admitido como tendo circulação concentrada ao longo de uma linha perpendicular ao plano do escoamento, conforme a figura 3.34 .

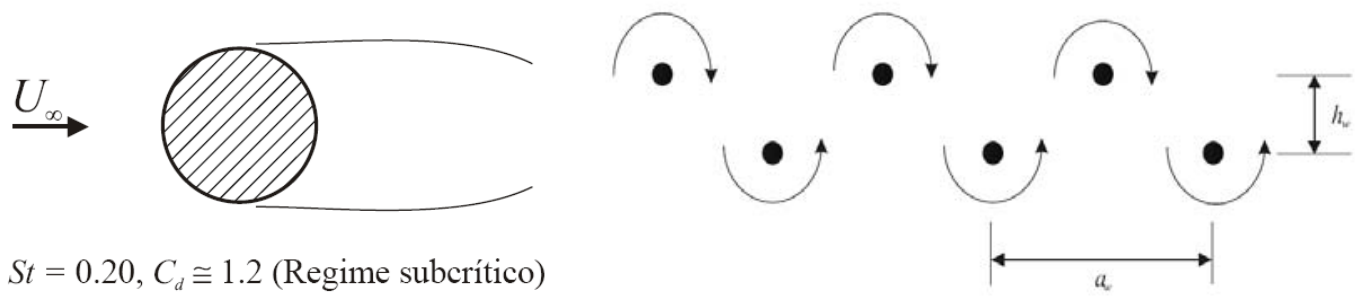

Figura 3.34 - Número de Strouhal (St) e coeficiente de arrasto (Cd) para um corpo cilindríco; fileira dupla de vórtices idealizada por Von Kármán (ver [56]).

Para o riser abordado na seção anterior, estuda-se, nesta seção, seu comportamento dinâmico quando consideradas vibrações induzidas por vórtices $(V I V)$. O exemplo de VIV aqui abordado não tem a intenção de representar as 
condições freqüentemente encontradas no campo, tendo por objetivo apenas mostrar as capacidades da técnica empregada neste trabalho. As equações que representam a dinâmica do fluido foram retiradas de Blevins [10] e Parra [67]. Admite-se que, para o seguinte número de Strouhal $S_{t}=\frac{f D}{U} \cong 0,20$, o primeiro modo seja excitado, ou seja, o lock-in (ver [69]) ocorre para o primeiro modo de vibração. Isto leva às equações 3.68 e 3.69. A equação 3.68 representa a dinâmica da estrutura e a equação 3.69 a dinâmica do fluido.

$$
\begin{gathered}
\ddot{U}+2,71 \times 10^{-2} U+8,14733 V+2,49 \times 10^{-4} U^{3}-2,23 \times 10^{-2} U V^{2}=8,01911 \dot{z}, \\
\dot{z}^{2}+0,0271 z=-19,9196 \dot{z}^{3}+0,06332 \dot{z}+0,40105 V .
\end{gathered}
$$

Nas equações 3.68 e $3.69, U$ e $V$ são, respectivamente, o deslocamento e a velocidades referentes ao grau de liberdade localizado na metade do comprimento do riser. Uma interpretação para a variável $z$, aqui utilizada para representar a dinâmica do fluido, é apresentada por Aranha [3], [4].

A figura 3.35 mostra a resposta no tempo do deslocamento $U$ para excitação por VIV. Ambas as respostas linear e não-linear praticamente coincidem, pois os valores da amplitude não são suficientes para que as não-linearidades sejam perceptíveis. A figura 3.36 mostra o diagrama de fase. É possível notar em ambas as figuras uma fase inicial transiente seguida pelo regime permanente com amplitude de 1,5m. Obviamente, este valor de amplitude é meramente ilustrativo e serve apenas para mostrar a robustez do modelo estrutural. Usualmente, valores de amplitude devido a VIV são da ordem de um diâmetro e meio a dois diâmetros do riser. 


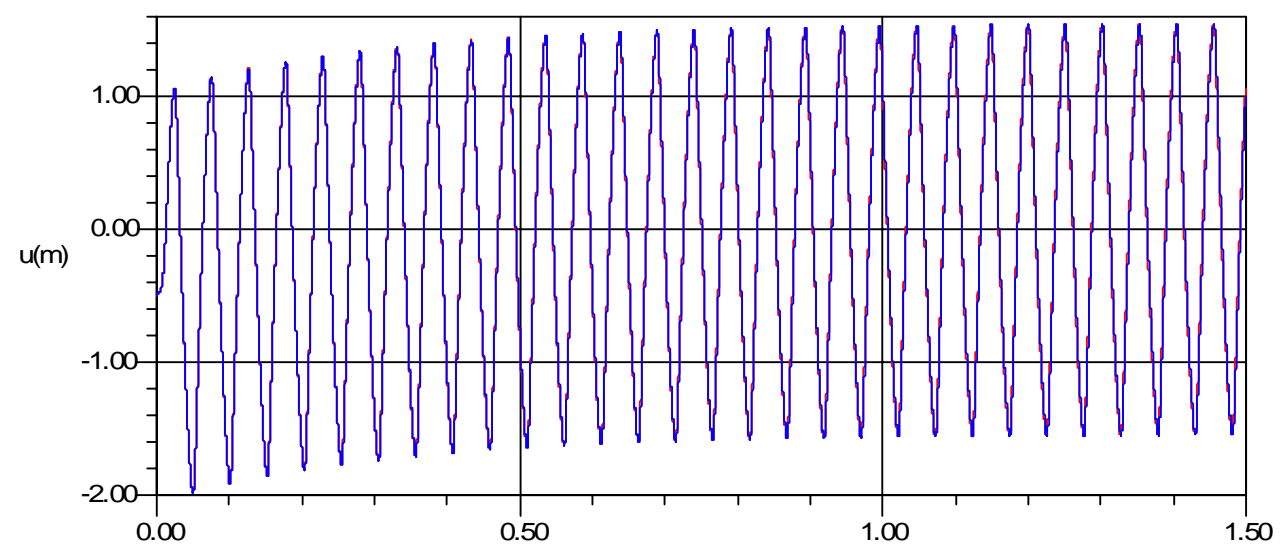

$0.00<\mathrm{X}<1.50 \mathrm{e}+3 ;-2.0<\mathrm{Y}<1.5 \quad \mathrm{t}(\mathrm{s})(103)$

Figura 3.35 - Resposta no tempo para VIV, riser reto - Linear (Azul) e Não-Linear (Vermelho).

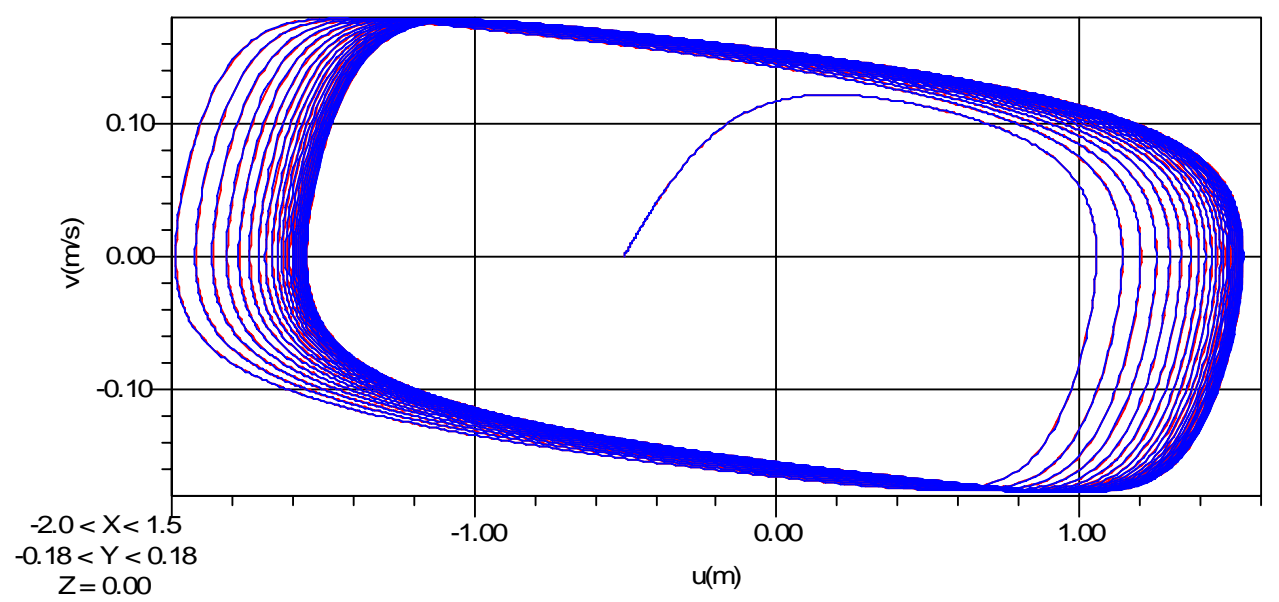

Figura 3.36 - Diagrama de fase para VIV, riser reto - Linear (Azul) e Não-Linear (Vermelho).

Vale notar que a construção dos modelos não-lineares de elementos finitos exige grande esforço computacional, alcançando o limite das máquinas disponíveis para a realização deste trabalho. Para fins acadêmicos, os resultados encontrados neste trabalho são adequados e permitem uma análise qualitativa dos fenômenos. No entanto, para aplicações específicas, mais desenvolvimentos se fazem necessários. 
Os resultados apresentados nesta tese sobre a dinâmica de risers excitados por $V I V$, não são conclusivos. Ao contrário, estes resultados têm o intuito de, apenas, mostrar as potencialidades da abordagem por modos não-lineares na representação adequada da dinâmica de risers e, se possível, apontar algumas direções para trabalhos futuros. 


\section{Capítulo 4}

\section{Risers em Catenária}

Neste capítulo estudam-se risers em catenária. A figura 4.1 apresenta um modelo esquemático de riser em catenária no plano. Os exemplos seguintes consistem em risers de aço em catenária ("Steel Catenary Riser - S.C.R.”) bi-dimensionais. Neste texto, a sigla "SCR" será utilizada para significar "Steel Catenary Riser". A figura 4.2 mostra o modelo de elementos finitos para os risers estudados neste capítulo. O grau de liberdade 50 é escolhido para coordenada generalizada modal. Os modos não-lineares serão determinados para dois exemplos. O primeiro se trata de um SCR com extremidades fixas (figura 4.3), denominado "SCR fixo-fixo". Para o segundo exemplo, altera-se a vinculação da extremidade superior do primeiro exemplo pela introdução de um sistema de amarração (figura 4.4), denomina-se este sistema como "SCR fixo-ancorado". Este sistema consiste na introdução de uma massa concentrada $M=151360$ ton e de uma mola de rigidez $K=90000 \mathrm{~N} / \mathrm{m}$. Esse sistema massa-mola representa um sistema de amarração mais uma embarcação. A tabela 4.1 apresenta as propriedades físicas para todos os SCR's deste capítulo.

\begin{tabular}{|l|c|}
\hline Módulo de Young & $E=2,1 \times 10^{11} \mathrm{~N} / \mathrm{m}^{2}$ \\
\hline Comprimento & $\ell=1800 \mathrm{~m}$ \\
\hline Área da Seção Transversal & $A=1,1021 \times 10^{-2} \mathrm{~m}^{2}$ \\
\hline Momento de Inércia & $I=4,72143 \times 10^{-5} \mathrm{~m}^{4}$ \\
\hline Força Normal Inicial (no topo) & $N_{0}=2 \times 10^{6} \mathrm{~N}$ \\
\hline $\begin{array}{l}\text { Massa por unidade de comprimento (água interna }+ \\
\text { massa adicionada) }\end{array}$ & $m=141,24 \mathrm{~kg} / \mathrm{m}$ \\
\hline Peso submerso do riser por unidade de comprimento & $p=727 \mathrm{~N} / \mathrm{m}$ \\
\hline Velocidade da corrente & $U=0,5 \mathrm{~m} / \mathrm{s}$ \\
\hline Coeficiente de arrasto & $C_{D}=1.2$ \\
\hline Peso específico da água do mar & $\rho_{a}=1025 \mathrm{~kg} / \mathrm{m}^{3}$ \\
\hline Diâmetro externo & $D=0,2032 \mathrm{~m}$ \\
\hline Espessura & $e=19,05 \mathrm{~mm}$ \\
\hline
\end{tabular}

Tabela 4.1 - Propriedades físicas do SCR, ver Pesce et al [72]. 


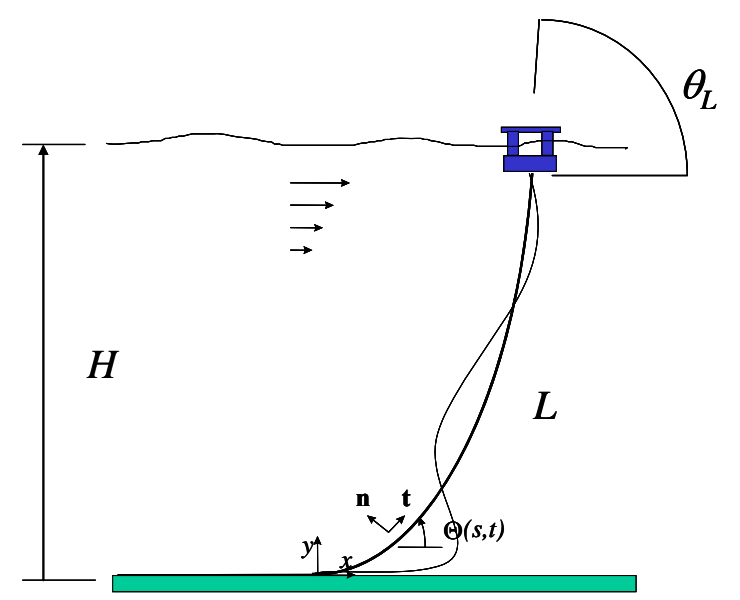

Figura 4.1 - Modelo esquemático de riser em catenária (bi-dimensional) ver [69].

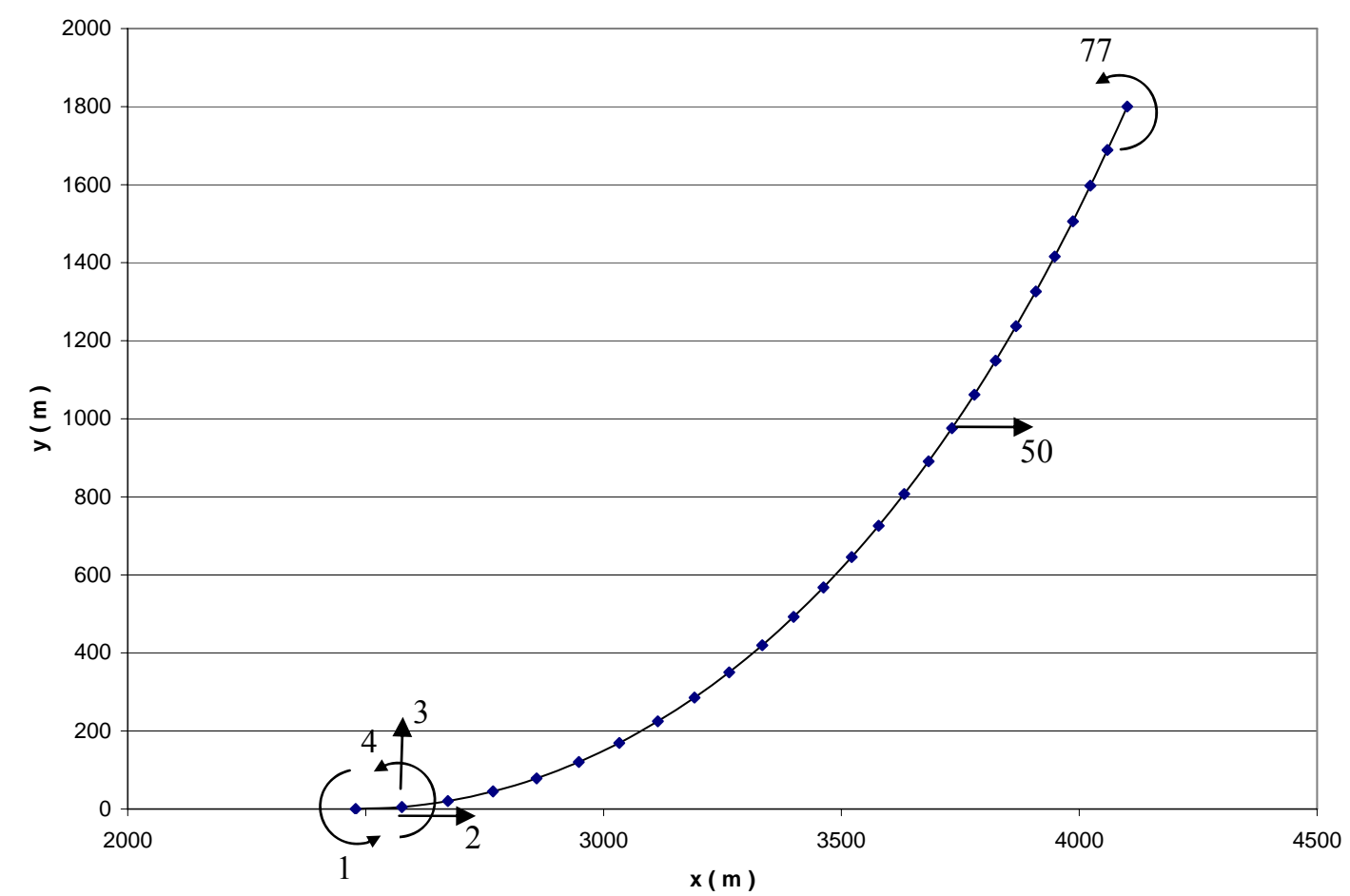

Figura 4.2 - Modelo de elementos finitos com numeração dos graus de liberdade. Configuração indeformada.O grau de liberdade 50 é o deslocamento modal. 


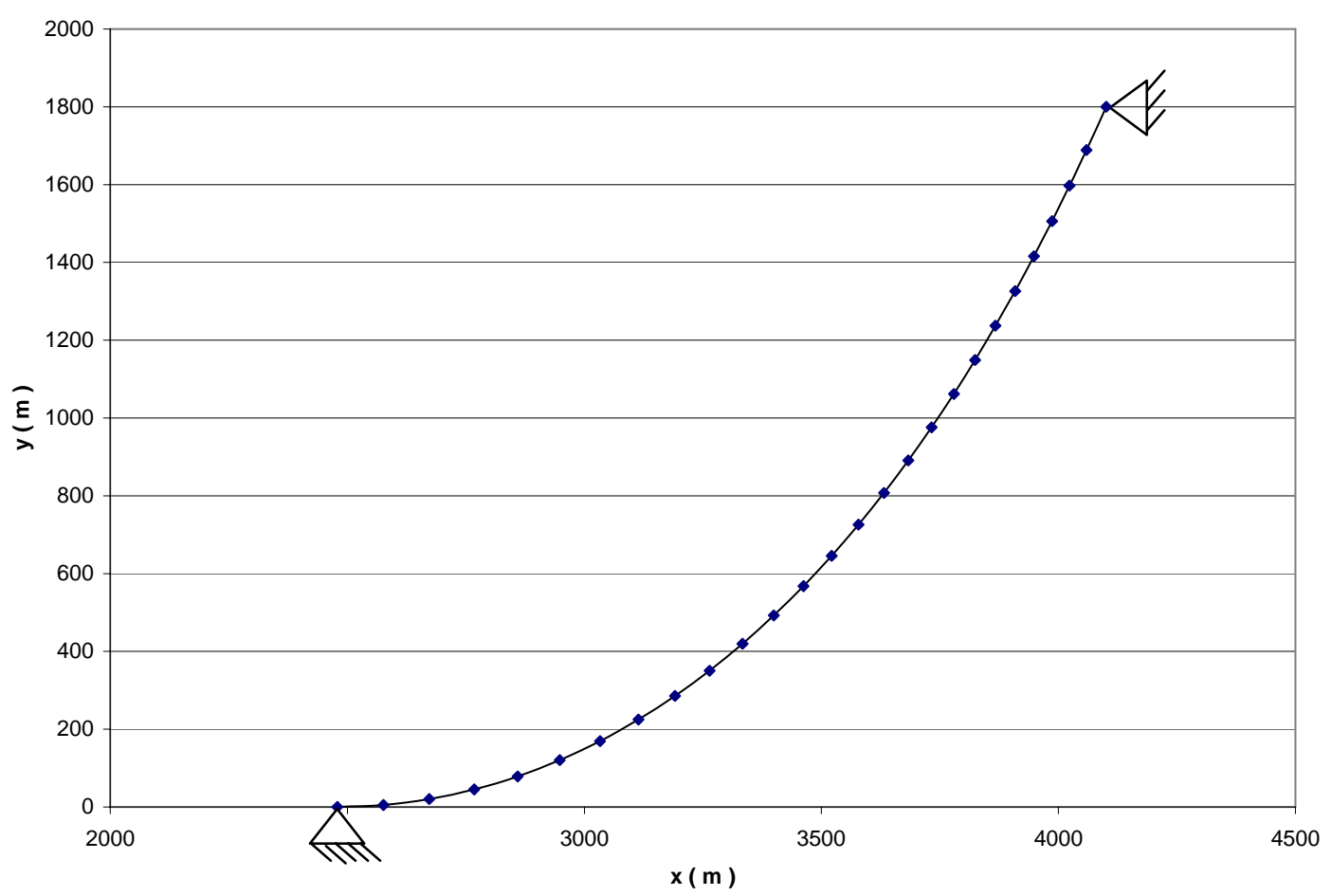

Figura $4.3-\quad$ SCR: condições de contorno para o caso fixo-fixo.

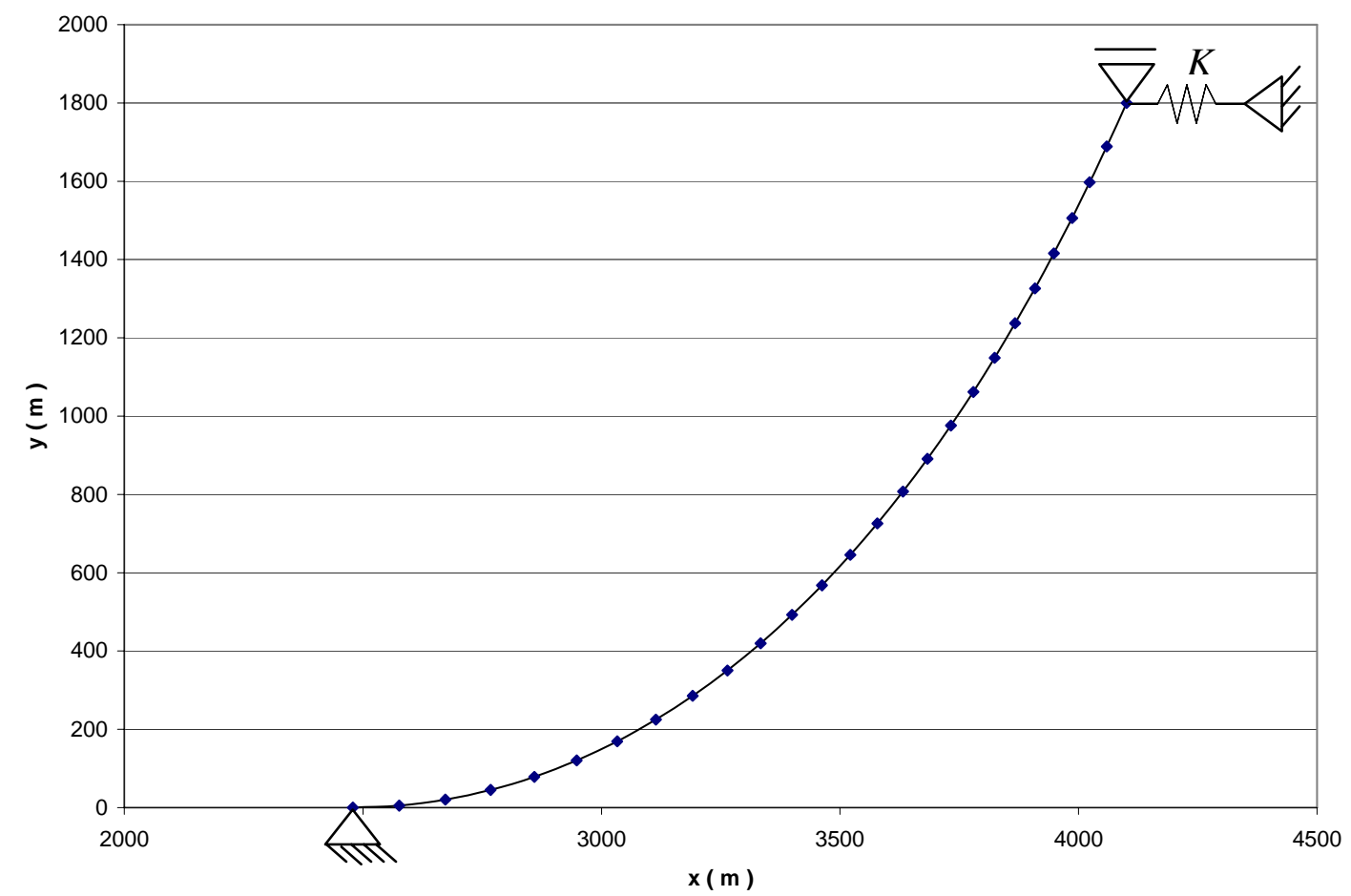

Figura $4.4-\quad$ SCR: condições de contorno para o caso fixo-ancorado. 
Valores típicos de períodos naturais de oscilação de uma plataforma semisubmersível ancorada em $1800 m$ de profundidade são da ordem de 250s, muito maiores que o primeiro período natural linear de um SCR que é da ordem de 30s. (ver figura 4.5). Desta forma, a dinâmica da ligação SCR-plataforma pode ser considerada quase-estática o que torna a hipótese de extremos fixos completamente adequada ver Pesce et al [72]. Uma correção posterior pode ser feita no TDP (sigla para "Touch Down Point") via Técnica da Camada Limite (ver Pesce et al [71]) ou uma mudança de variáveis, que transforme o problema de contornos móveis em contornos fixos, ver Mazzilli [52].

É possível observar na figura 4.5 as freqüências naturais de um SCR proposto por Pesce et al [72]. Apresentam-se três soluções numéricas com diferentes valores de EA e uma analítica (WKB - Wentzel-Kramers-Brillouin), ver [9]. Ressalta-se a relação praticamente linear entre o número do modo e a freqüência natural. Tal comportamento aponta para possíveis ressonâncias internas. Portanto, os modos nãolineares poderão ser uma ferramenta interessante para o entendimento e descrição destes fenômenos de acoplamento em SCR's. Estas evidências motivam os estudos dos modos não-lineares nos exemplos que se seguem.

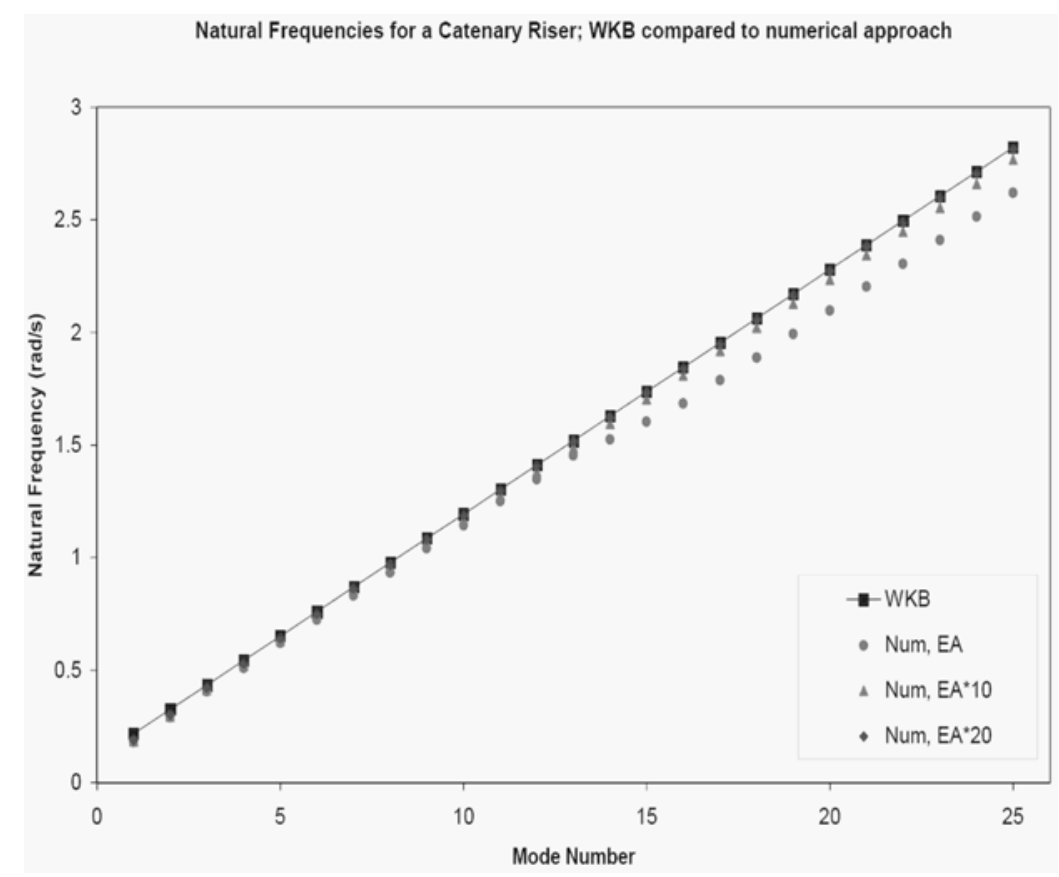

Figura $4.5-\quad$ Freqüências naturais de um SCR segundo Pesce et al [72]. 


\subsection{Fixo-Fixo}

Esta seção estuda o primeiro modo não-linear do riser apresentado na figura 4.3. Na figura 4.6, observa-se o primeiro modo linear e o primeiro modo não-linear do sistema estrutural. Nota-se no caso linear um ponto de inflexão, enquanto que no modo não-linear encontram-se dois pontos de inflexão. Esta constatação evidencia a influência do terceiro modo linear no primeiro modo não-linear. Esta capacidade de um modo não-linear ser influenciado por outro modo superior também pode ser notada para risers retos e está relacionada à participação de não-linearidades. Tais fenômenos reafirmam a importância dos modos não-lineares na compreensão da dinâmica desses sistemas estruturais.

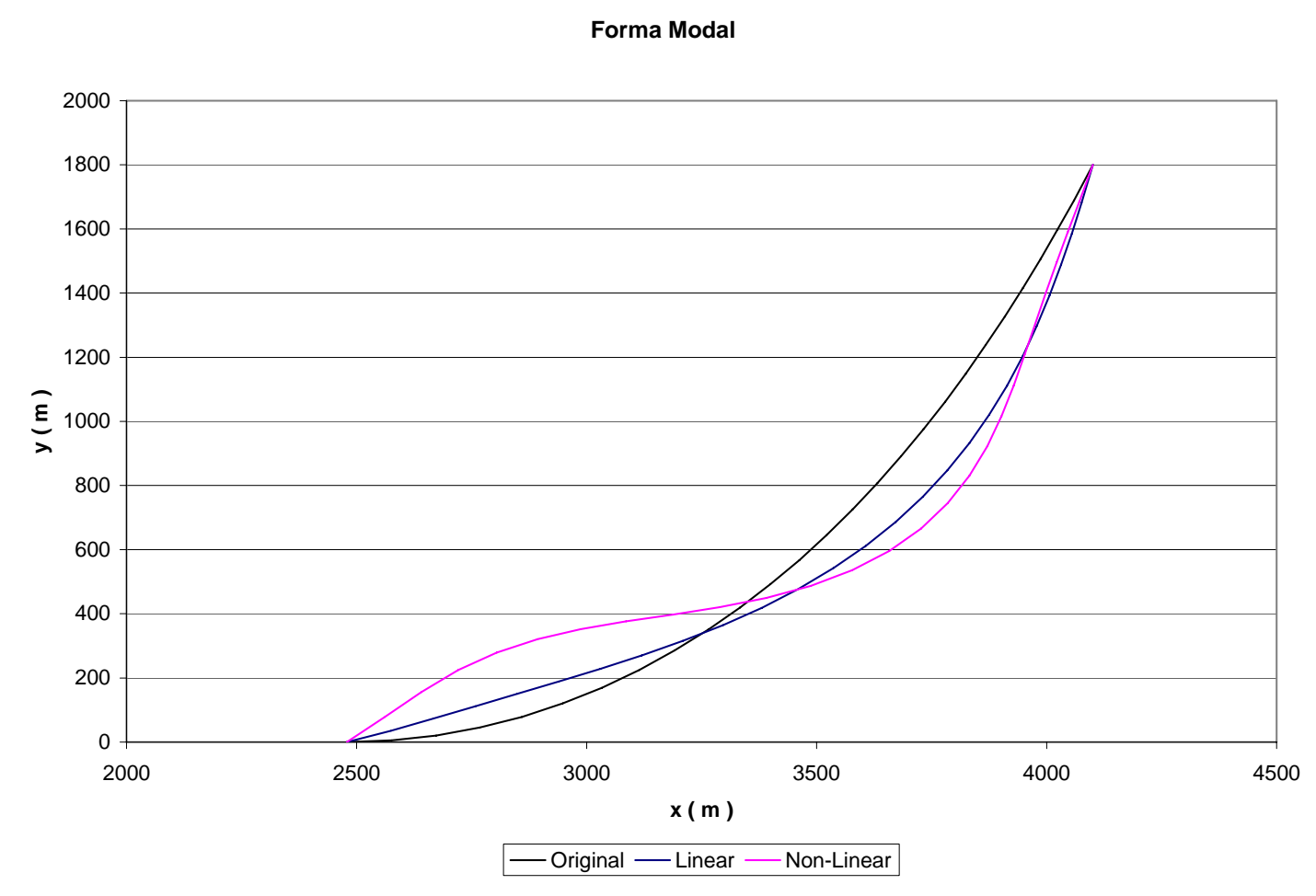

Figura $4.6-\quad$ Forma modal do primeiro modo; amplitude inicial $=100 \mathrm{~m}$. 
Um importante efeito não-linear da curvatura estática inicial do riser é apresentado nas figuras 4.7 e 4.8: a resposta não-simétrica da amplitude. Para os exemplos do capítulo anterior, o deslocamento máximo é igual em módulo ao mínimo deslocamento. Porém, no caso do "SCR", a geometria assimétrica leva a uma rigidez estrutural também não-simétrica. Tal assimetria é introduzida pelos termos em $U^{2} \mathrm{e}$ $V^{2}$ que eram ausentes nos risers retos.

A equação não-linear do oscilador modal (primeiro modo) para o "SCR" de extremos fixos é:

$$
\ddot{U}+3,46 \times 10^{-2} U+8,17 \times 10^{-5} U^{2}+5,38 \times 10^{-3} V^{2}+3,52 \times 10^{-7} U^{3}+5^{5}, 78 \times 10^{-5} U V^{2}=0 .
$$

Nota-se que o período da solução não-linear é menor que o da solução linear indicando um aumento da freqüência e, portanto, um aumento da rigidez para o sistema não-linear. Observando a equação 4.1 verifica-se que ambos os termos $U^{3}$ e $U V^{2}$ contribuem para o aumento da rigidez.

No intuito de evidenciar ainda mais os efeitos não-lineares, a figura apresenta o mesmo "SCR" de extremos fixos com uma amplitude modal inicial (grau de liberdade 50 ver figura 4.2) de $200 \mathrm{~m}$ (a velocidade modal inicial é nula para todos os exemplos deste capítulo). Apesar do valor de amplitude exagerado, o modelo baseado nas variedades invariantes mostra-se robusto.

Os dois pontos de inflexão agora são bastante evidentes. Uma forte deformação do diagrama de fase indica a grande participação das não-linearidades. A tendência de enrijecimento fica óbvia com a queda ainda maior do período não-linear. A assimetria dos deslocamentos máximos e mínimos se intensifica como esperado, já que quanto maiores as amplitudes, maiores as participações dos termos não-lineares. 


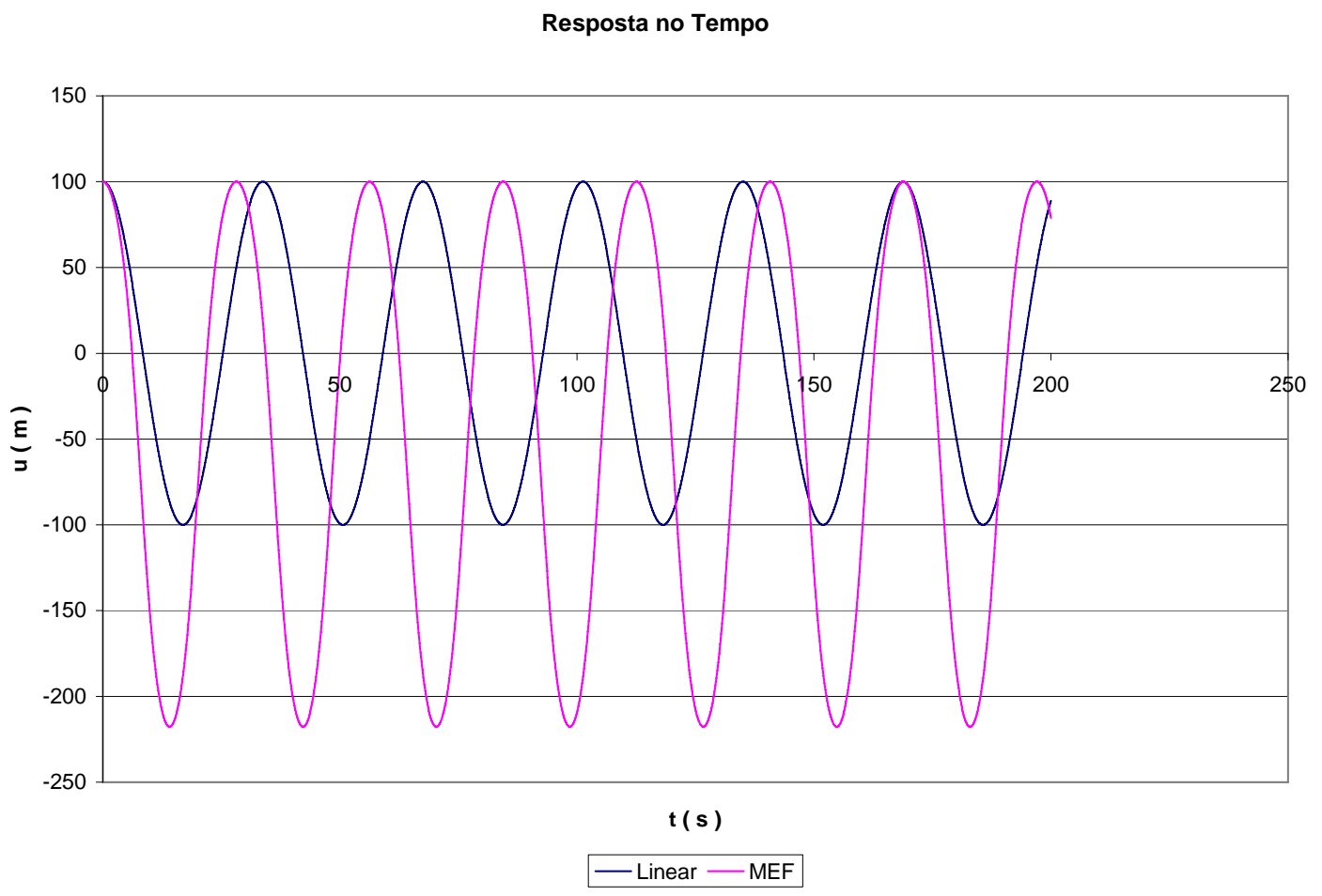

Figura 4.7- Riser em catenária; primeiro modo; amplitude inicial de $100 \mathrm{~m}^{*}$. Períodos: 33,76 s (linear); 28,15 s (MEF).

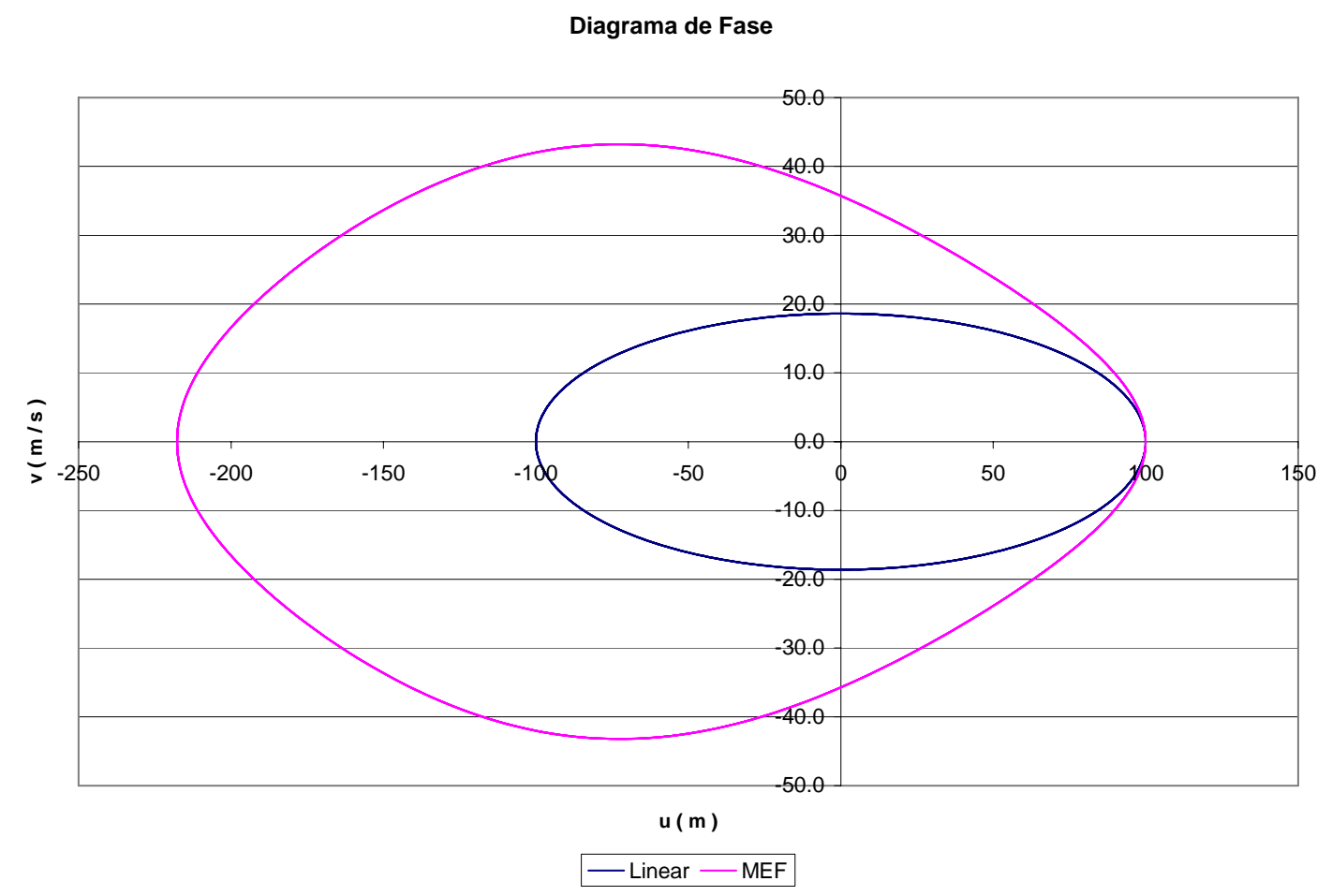

Figura 4.8 - Diagrama de fase para o primeiro modo; amplitude inicial de $100 \mathrm{~m}$.

\footnotetext{
* Os valores de amplitude e velocidade não condizem com os valores comumente encotrados em aplicações práticas e são aqui utilizados apenas para estudo dos fenômenos não-lineares e robustez dos modelos matemáticos e computacionais.
} 


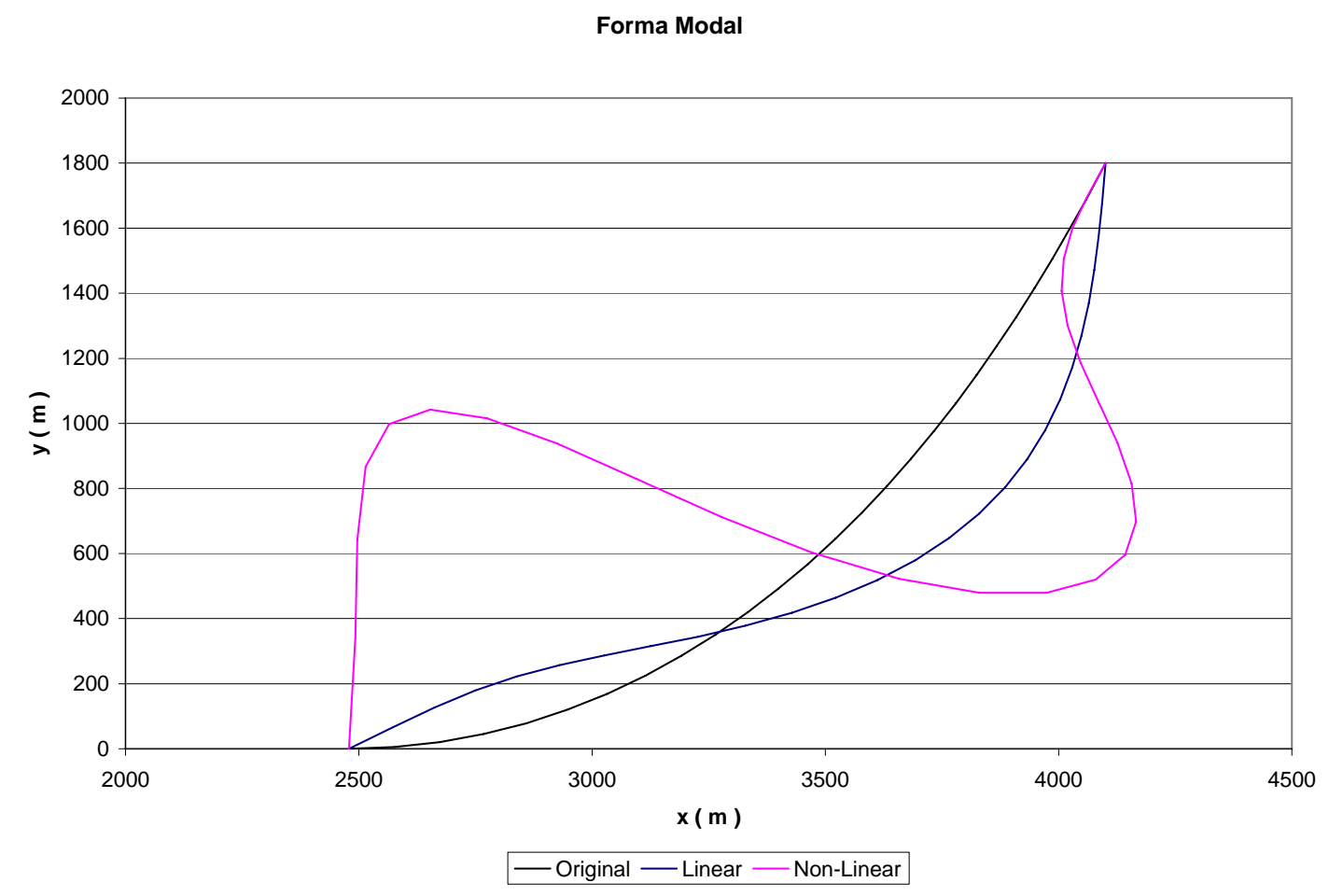

Figura $4.9-\quad$ Forma modal do primeiro modo; amplitude inicial $=200 \mathrm{~m}^{\dagger}$.

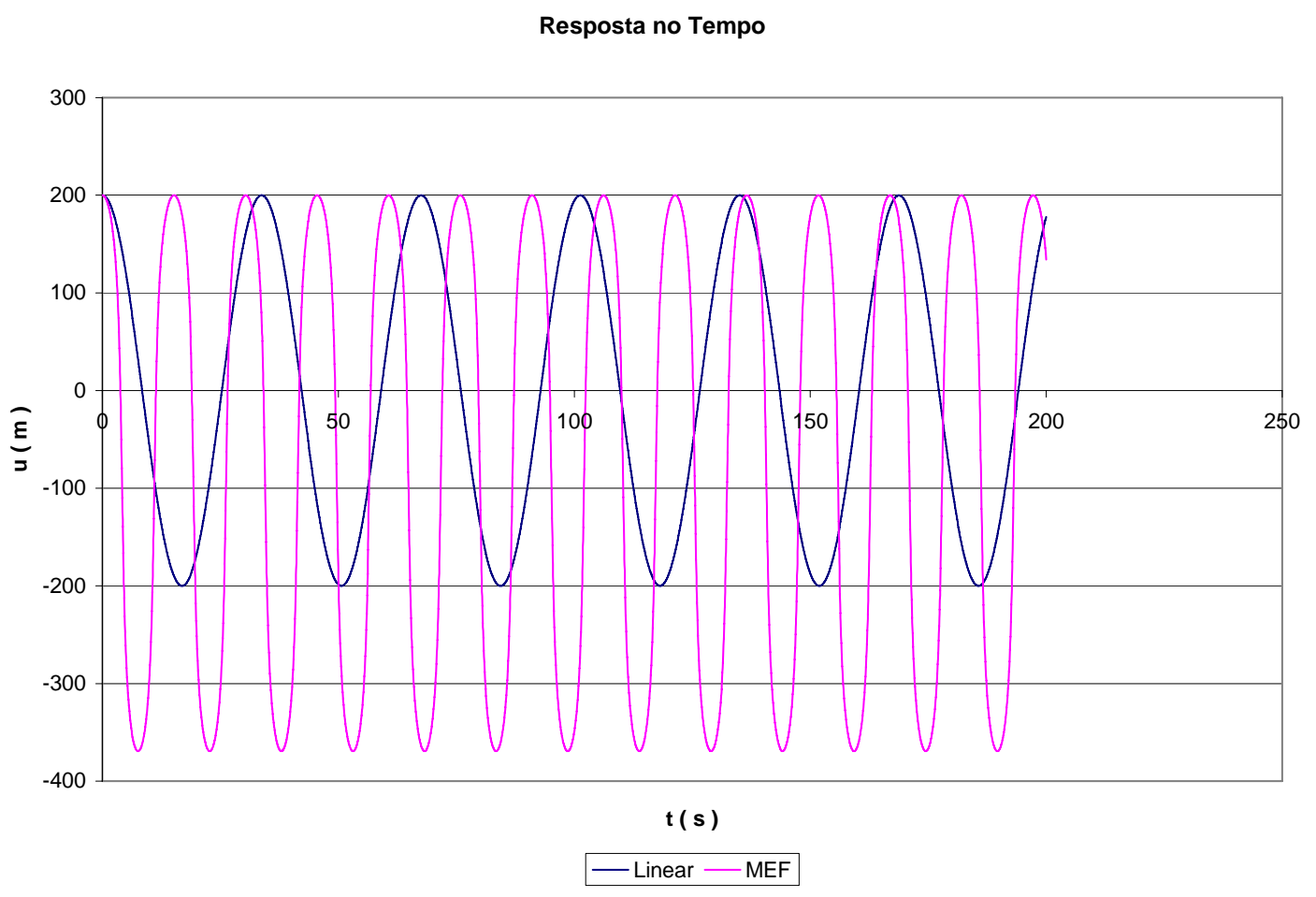

Figura 4.10- Riser em catenária; primeiro modo; amplitude inicial de $200 \mathrm{~m}$. Períodos: 33,76 s (linear); 15,17 s (MEF).

\footnotetext{
${ }^{\dagger}$ Os valores de amplitude e velocidade não condizem com os valores comumente encotrados em aplicações práticas e são aqui utilizados apenas para estudo dos fenômenos não-lineares e robustez dos modelos matemáticos e computacionais.
} 


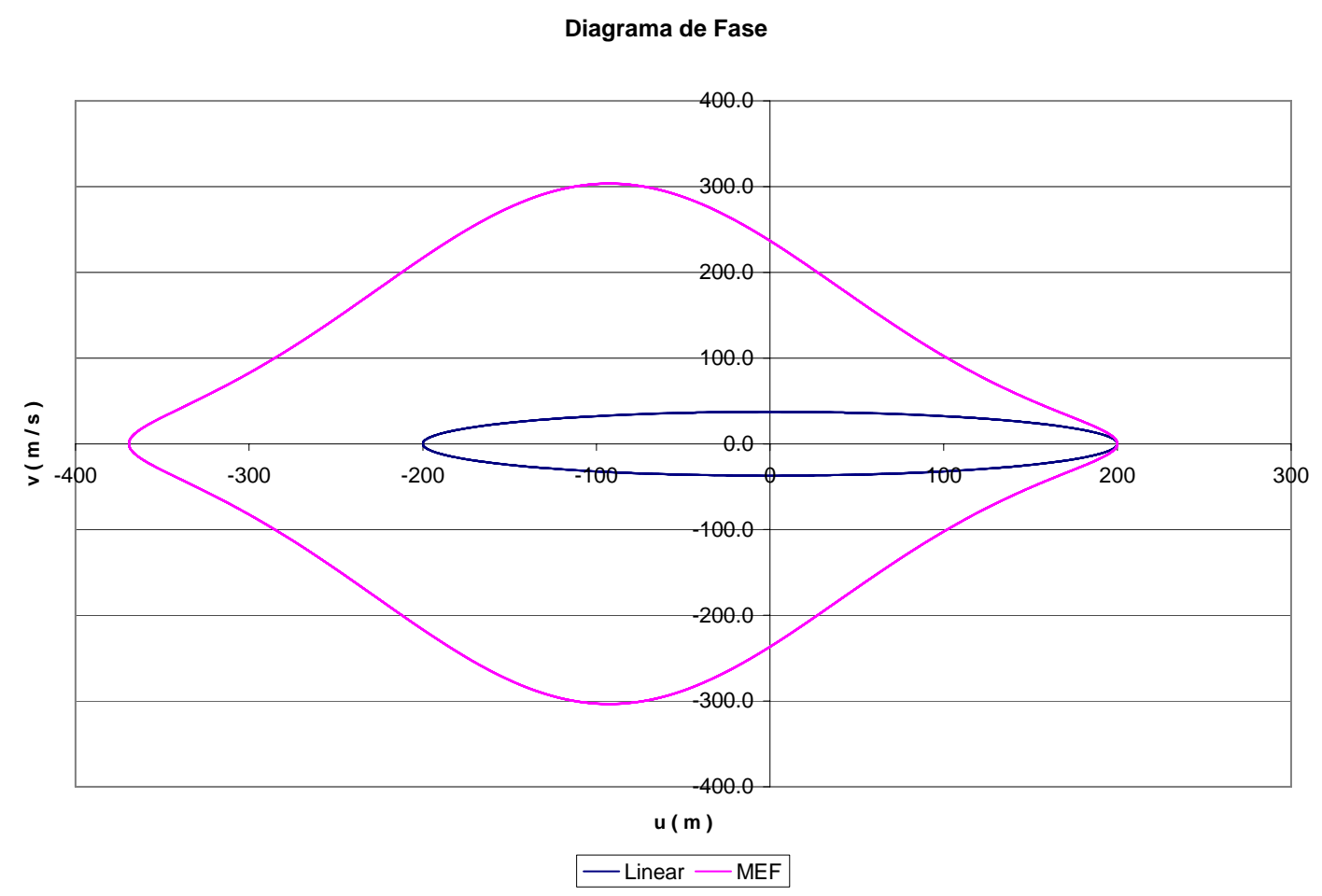

Figura 4.11 - Diagrama de fase para o primeiro modo; amplitude inicial de $200 \mathrm{~m}^{\ddagger}$.

\subsubsection{Vibração Induzida por Vórtices - Riser em Catenária}

Para o SCR fixo-fixo abordado na seção anterior, estuda-se a dinâmica devida às vibrações induzidas por vórtices (VIV). Admitindo-se o seguinte número de Strouhal $S_{t}=\frac{f D}{U} \cong 0,20$ e a velocidade da corrente $U=0,5 \mathrm{~m} / \mathrm{s}$ (perpendicular ao plano do movimento), o lock-in ocorre para o modo de vibração 26. Isto leva às equações 4.2 e 4.3. O lock-in é a sintonia entre a freqüência de liberação de vórtices e uma das freqüências naturais de oscilação do riser. Esta sintonia tende a amplificar a oscilação da linha. No entanto, esta mesma oscilação acaba por destruir o padrão de escoamento, auto-limitando as amplitudes do movimento, ver [69]. As equações que representam a dinâmica do fluido foram retiradas de Blevins [10] e Parra [67]. A equação 4.2 representa a dinâmica da estrutura e a equação 4.3 a dinâmica do fluido.

$$
\ddot{U}+8,1921 U+39 V+22,216 U^{2}-3,0673 V^{2}-70,823 U^{3}+533,54 U V^{2}=38,95 \dot{z} .
$$

\footnotetext{
* Os valores de amplitude e velocidade não condizem com os valores comumente encotrados em aplicações práticas e são aqui utilizados apenas para estudo dos fenômenos não-lineares e robustez dos modelos matemáticos e computacionais.
} 


$$
\dot{z}^{2}+9,8696 z=-4,17 z^{3}+0,3125 \dot{z}+1,98 V .
$$

Nas equações 4.2 e 4.3, $U$ e $V$ são, respectivamente, o deslocamento e a velocidades referentes ao grau de liberdade 50. A variável $z$ é utilizada para representar a dinâmica do fluido.

A figura 4.12 mostra a resposta no tempo para o deslocamento do grau de liberdade 50. Nota-se um regime transiente inicial seguido do regime permanente. No regime permanente, apesar da amplitude total do sistema não-linear $(0,565 \mathrm{~m})$ não ser muito diferente da amplitude do sistema linear $(0,567 \mathrm{~m})$, é possível observar na resposta não-linear que a amplitude máxima $(0,3057 \mathrm{~m})$ é, em módulo, diferente da amplitude mínima $(0,2596)$. Tal comportamento está relacionado à assimetria geométrica do sistema estrutural. A resposta linear apresenta extremos iguais a $+0,2834 m$ e $-0,2834 m$, no regime permanente.

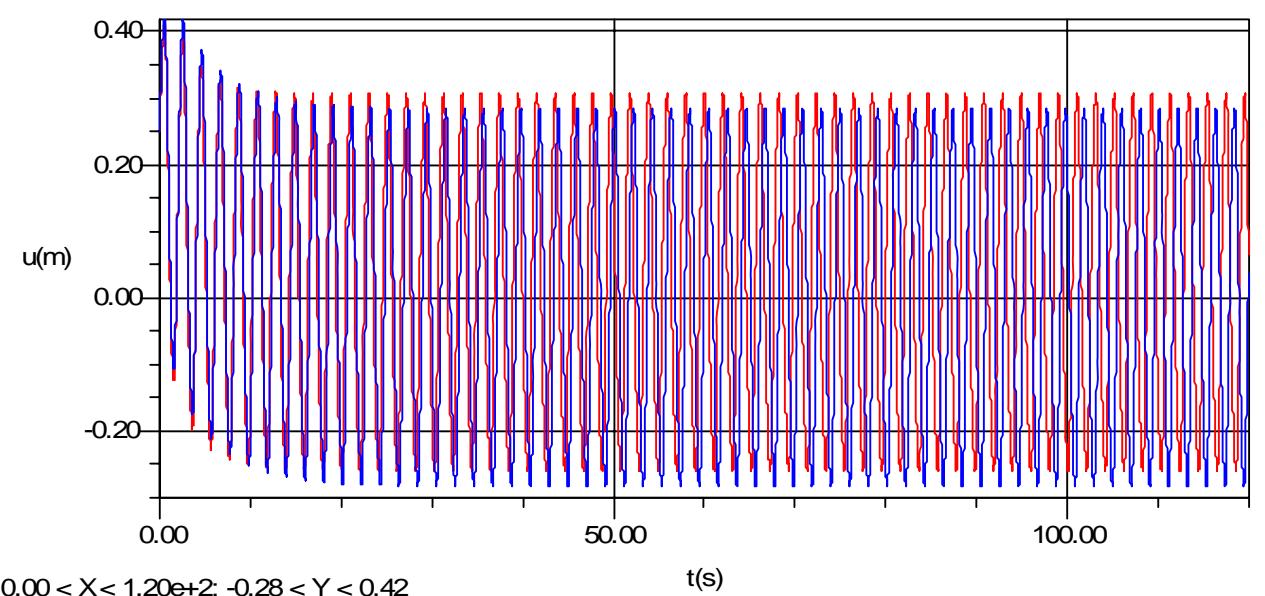

Figura 4.12 - Resposta no tempo, SCR - Linear (Azul) e Não-Linear (Vermelho). 


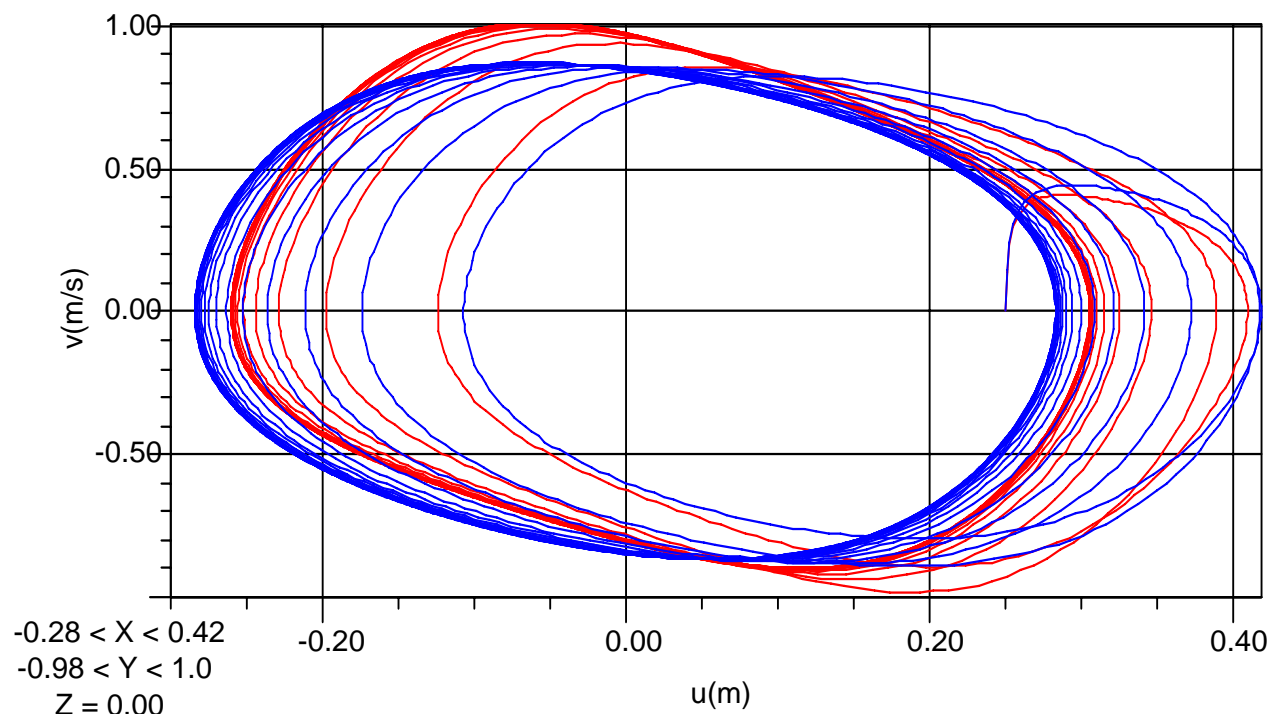

Figura 4.13 - Diagrama de fase, SCR - Linear (Azul) e Não-Linear (Vermelho).

O mesmo comportamento com relação às amplitudes dos deslocamentos é observado na figura 4.13. Além disso, verifica-se a assimetria das velocidades do modelo não-linear e, também, valores de velocidades superiores àqueles encontrados na resposta linear. A amplitude do deslocamento é pequena, no entanto, mas é possível notar as primeiras influências das não-linearidades.

Os resultados aqui apresentados sobre a dinâmica de risers excitados por VIV, não são conclusivos e têm o intuito de, apenas, mostrar as potencialidades do método. 


\subsection{Fixo-Ancorado}

Nesta seção estuda-se o "SCR" fixo-ancorado da figura 4.4, sendo a massa concentrada $M=151360$ ton e a mola de rigidez $K=90000 \mathrm{~N} / \mathrm{m}$. A massa e a mola modelam a plataforma e seu sistema de amarração. A figura 4.14 apresenta o primeiro modo de vibração (linear e não-linear) que é alvo de estudo a seguir.

A equação não-linear do oscilador modal (primeiro modo) para o "SCR" de extremos fixo-ancorado é:

$$
\ddot{U}+3,46 \times 10^{-2} U+1,18 \times 10^{-5} U^{2}+3,36 \times 10^{-3} V^{2}-3,19 \times 10^{-7} U^{3}+3,47 \times 10^{-5} U V^{2}=0 .
$$

As figuras 4.15 e 4.16 são encontradas a partir da integração da equação 4.4 pelo método de Runge-Kutta. Observa-se a assimetria dos deslocamentos máximos e mínimos já abordada na seção anterior. No entanto, esta assimetria é menor para o "SCR" fixo-ancorado $U_{\text {máx }}=100 m$ e $U_{\text {mín }}=-171,29 m$ se comparado ao "SCR" fixo-fixo $U_{\text {máx }}=100 \mathrm{~m}$ e $U_{\text {mín }}-217,69 \mathrm{~m}$. Explica-se esse fenômeno pela presença de $M$ e $K$ (plataforma mais sistema de ancoragem) que absorvem parte da energia do sistema estrutural.

Nota-se também um aumento do período do sistema não-linear (34.86s) se comparado ao período natural (33,76s). Novamente, $M$ e $K$ têm papel importante no fenômeno do aumento do período, pois aumentam a flexibilidade e a massa modal do sistema estrutural, diminuindo a freqüência e, portanto, elevando o período.

Observando-se a equação do oscilador modal 4.4 é possível verificar que os termos $U^{3}$ e $U V^{2}$ contribuem contrariamente para a rigidez do sistema: o primeiro flexibiliza e o segundo enrijece. O termo vencedor depende dos valores da amplitude inicial e da velocidade inicial. Portanto, neste caso, não é trivial identificar uma tendência ao enrijecimento ou à flexibilização. Os termos em $U^{2}$ e $V^{2}$ estão novamente presentes garantindo a assimetria da resposta. 


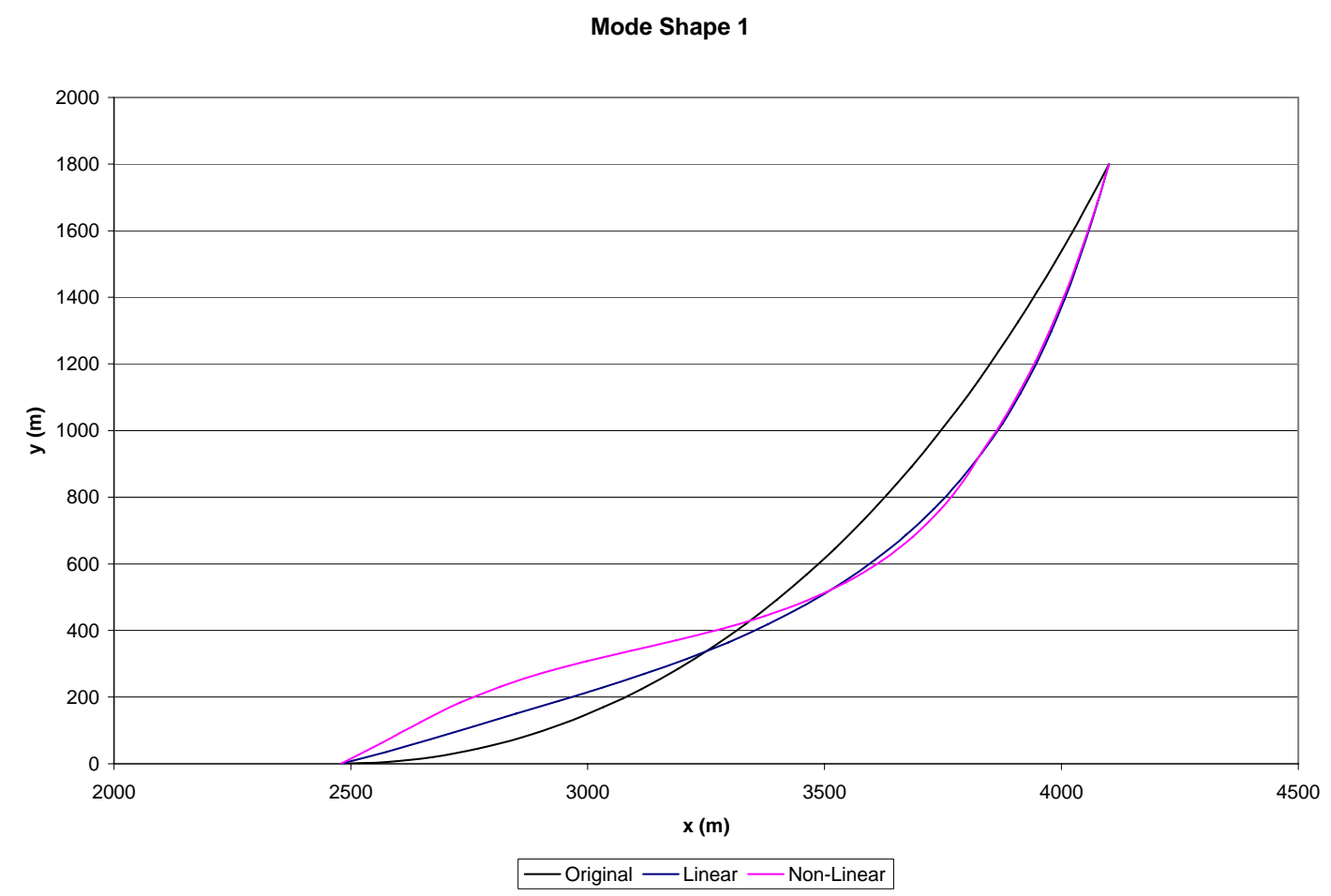

Figura $4.14-$ Forma modal do primeiro modo; amplitude inicial $=100 \mathrm{~m}$.

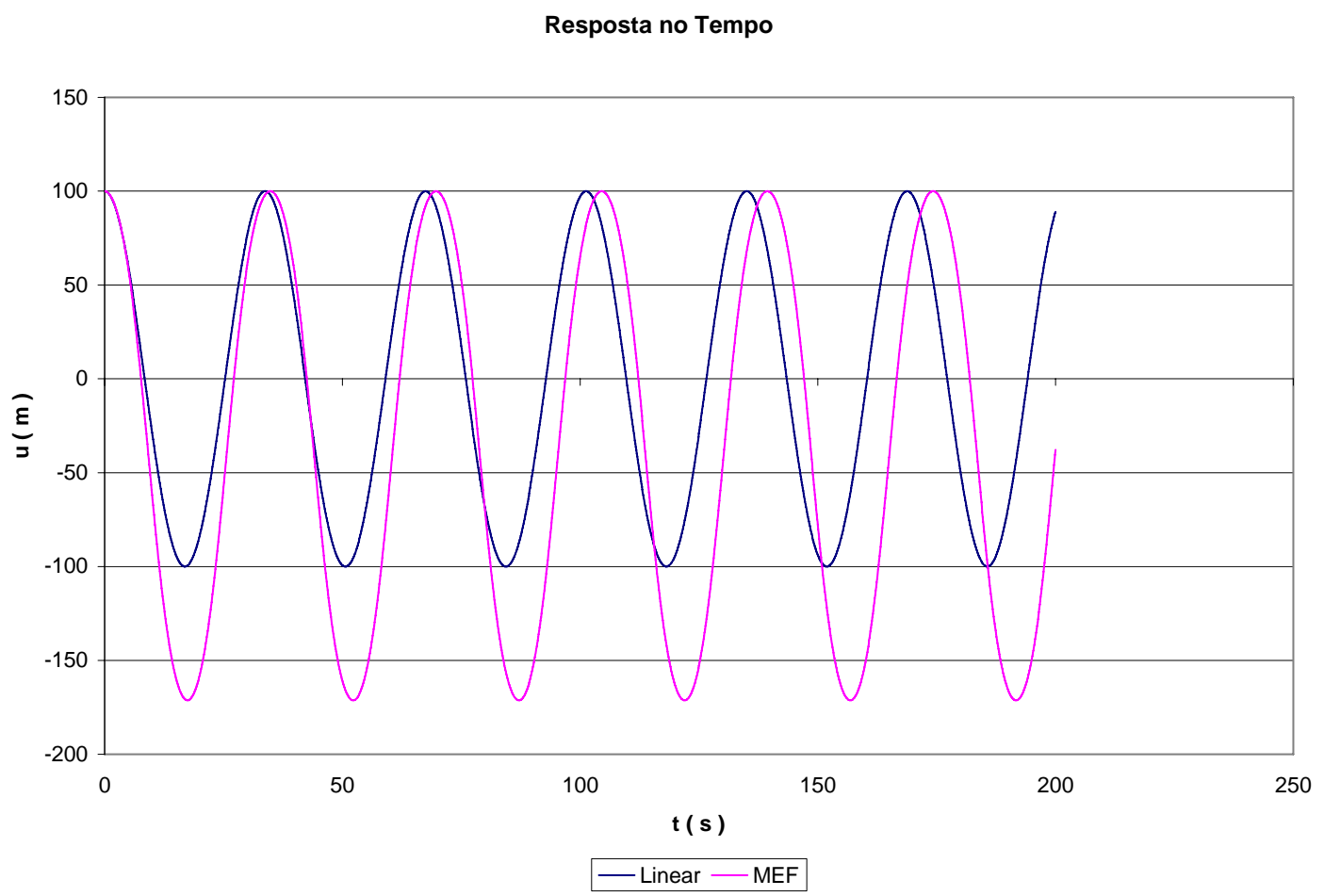

Figura 4.15 - Riser em catenária; primeiro modo; amplitude inicial de $100 \mathrm{~m}^{\S}$. Períodos: 33,76 s (linear); 34.86 s (MEF).

\footnotetext{
$\S$ Os valores de amplitude e velocidade não condizem com os valores comumente encotrados em aplicações práticas e são aqui utilizados apenas para estudo dos fenômenos não-lineares e robustez dos modelos matemáticos e computacionais.
} 
Diagrama de Fase

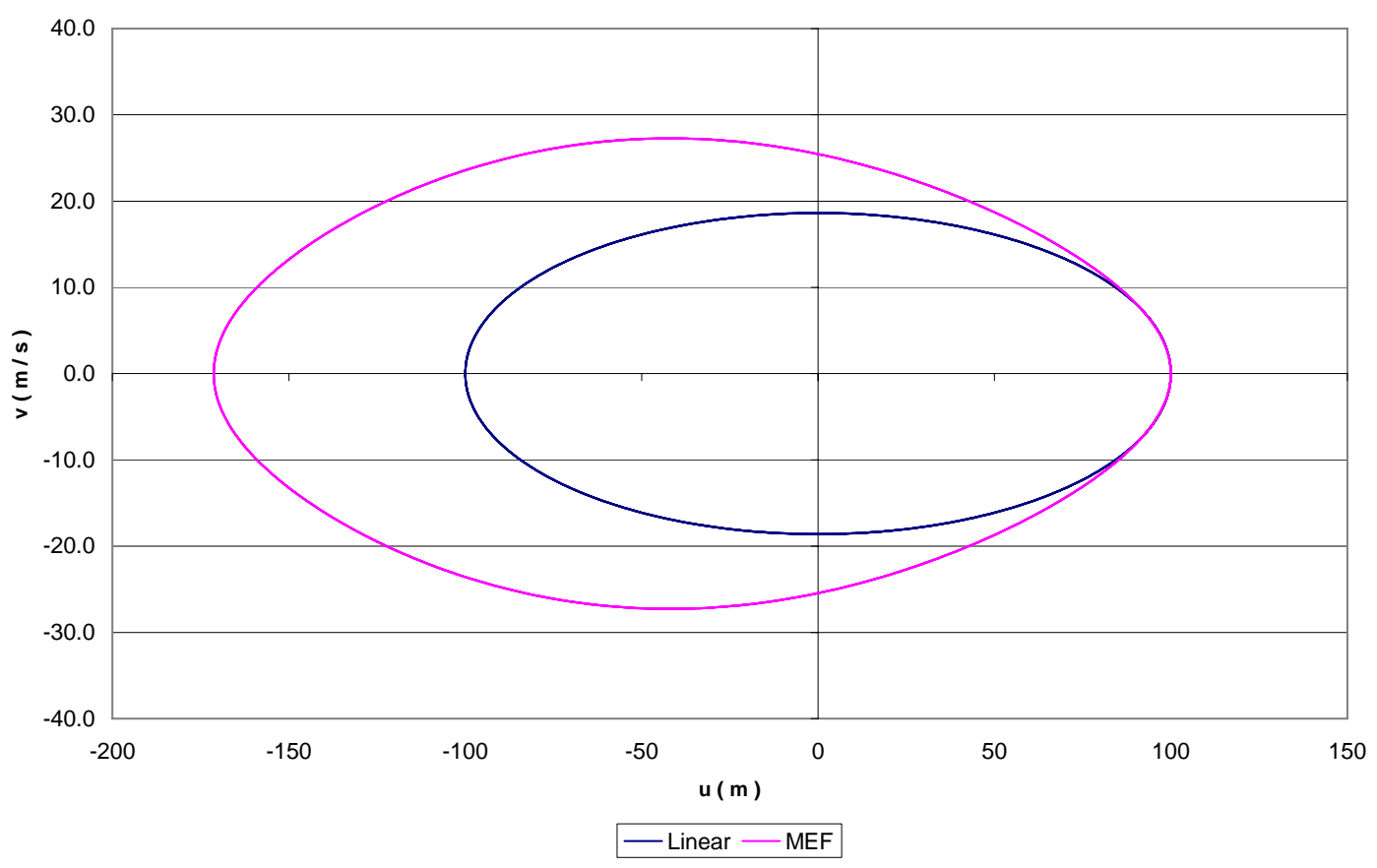

Figura 4.16 - Diagrama de fase para o primeiro modo; amplitude inicial de $100 \mathrm{~m}$. 


\section{Capítulo 5}

\section{Controle Ativo}

Este capítulo apresenta o estudo de controle ativo para os exemplos de risers dos capítulos 3 e 4 . O método de controle é o da alocação de pólos. O observador utilizado é o de Luenberger, ver [44]. Neste texto estuda-se apenas o projeto da lógica do sistema de controle que diz respeito à matriz de ganho do sistema, à matriz de ganho do observador e os valores ótimos dessas matrizes, ver Ogata [65]. Não é objeto de estudo o projeto das instalações físicas (atuadores e sensores) e nem sua viabilidade. Serão estudadas basicamente as vibrações livres e apenas introdutoriamente as vibrações induzidas por vórtices.

Neste estudo será controlado apenas o primeiro modo não-linear. Serão utilizadas as equações dos osciladores modais obtidas em capítulos anteriores. Serão utilizados ganho linear e observador, também linear, apesar de o sistema estrutural ser não-linear. A utilização de um observador não-linear abre a janela de estabilidade e, claramente, é um tema possível para estudos futuros. Desconsideram-se os demais modos, pois um único modo não-linear já introduz uma complexidade considerável ao problema enfrentado. Além disso, a inclusão de muitos modos no projeto de controle eleva as dimensões das matrizes. Essa elevação requer um maior tempo de processamento retardando a resposta do sistema de controle. No entanto, futuramente, a introdução de mais modos não-lineares de vibração, ou mesmo multi-modos, se faz necessária para evitar que ao controlar um modo outros sejam excitados, por exemplo, por ressonâncias internas.

Destaca-se nesse texto a utilização de controle ótimo para alocação dos pólos. Utiliza-se o Regulador Linear Quadrático Determinístico (Linear Quadratic Regulator - LQR). Esse método consiste na minimização de funcional quadrático de custo. As expressões finais, demonstrações e implementação computacional são apresentadas. 
Nesta seção realiza-se o estudo de controle para os risers do capítulo 3 . Primeiramente, aborda-se a teoria considerando sistemas lineares e posteriormente estendem-se os conceitos para os sistemas não-lineares.

Considere-se a seguinte equação linear matricial de movimento originária do método dos elementos finitos,

$$
\mathbf{m} \ddot{\mathbf{q}}+\mathbf{k} \mathbf{q}=\mathbf{n} u
$$

onde $\mathbf{m}$ é a matriz de massa, $\mathbf{k}$ é a matriz de rigidez do sistema, $\mathbf{q}$ é o vetor dos deslocamentos generalizados e $u$ é a força introduzida pelo atuador. As matrizes $\mathbf{m}$ e $\mathbf{k}$ têm dimensão $n x n$ e os vetores $\mathbf{q}$ e $\mathbf{n}$ têm dimensão $n x 1$, com $n$ sendo o número de graus de liberdade. $\mathrm{O}$ vetor $\mathbf{n}$ é vetor posição da força do atuador.

Fazendo a transformação para o espaço de configuração modal vem,

$$
\mathbf{T}^{t} \mathbf{m} \mathbf{T} \ddot{\mathbf{z}}+\mathbf{T}^{t} \mathbf{k} \mathbf{T} \mathbf{z}=\mathbf{T}^{t} \mathbf{n} u
$$

onde, $\mathrm{T}$ é matriz dos autovetores do sistema normalizada em relação à matriz de massa.

Da expressão 5.2 vem,

$$
\mathbf{I} \ddot{\mathbf{z}}+\mathbf{K} \mathbf{z}=\mathbf{b} u
$$

onde, $\mathbf{K}=\mathbf{T}^{t} \mathbf{k} \mathbf{T}$ e $\mathbf{b}=\mathbf{T}^{t} \mathbf{n}$.

Fazendo a transformação do sistema de equações diferenciais de segunda ordem para primeira ordem tem-se, no espaço de estados,

$$
\left\{\begin{array}{l}
\ddot{\mathbf{z}} \\
\dot{\mathbf{z}}
\end{array}\right\}=\left[\begin{array}{cc}
\mathbf{0} & -\mathbf{K} \\
\mathbf{I} & \mathbf{0}
\end{array}\right]\left\{\begin{array}{l}
\dot{\mathbf{z}} \\
\mathbf{z}
\end{array}\right\}+\left\{\begin{array}{l}
\mathbf{b} \\
\mathbf{0}
\end{array}\right\} u
$$


ou ainda,

$$
\dot{\mathbf{x}}=\mathbf{A} \mathbf{x}+\mathbf{B} u
$$

onde,

$\mathbf{x}=\left\{\begin{array}{l}\dot{\mathbf{z}} \\ \mathbf{z}\end{array}\right\}, \quad \mathbf{A}=\left[\begin{array}{cc}\mathbf{0} & -\mathbf{K} \\ \mathbf{I} & \mathbf{0}\end{array}\right], \quad \mathbf{B}=\left\{\begin{array}{l}\mathbf{b} \\ \mathbf{0}\end{array}\right\}$.

O sistema de controle é apresentado na 5.1.

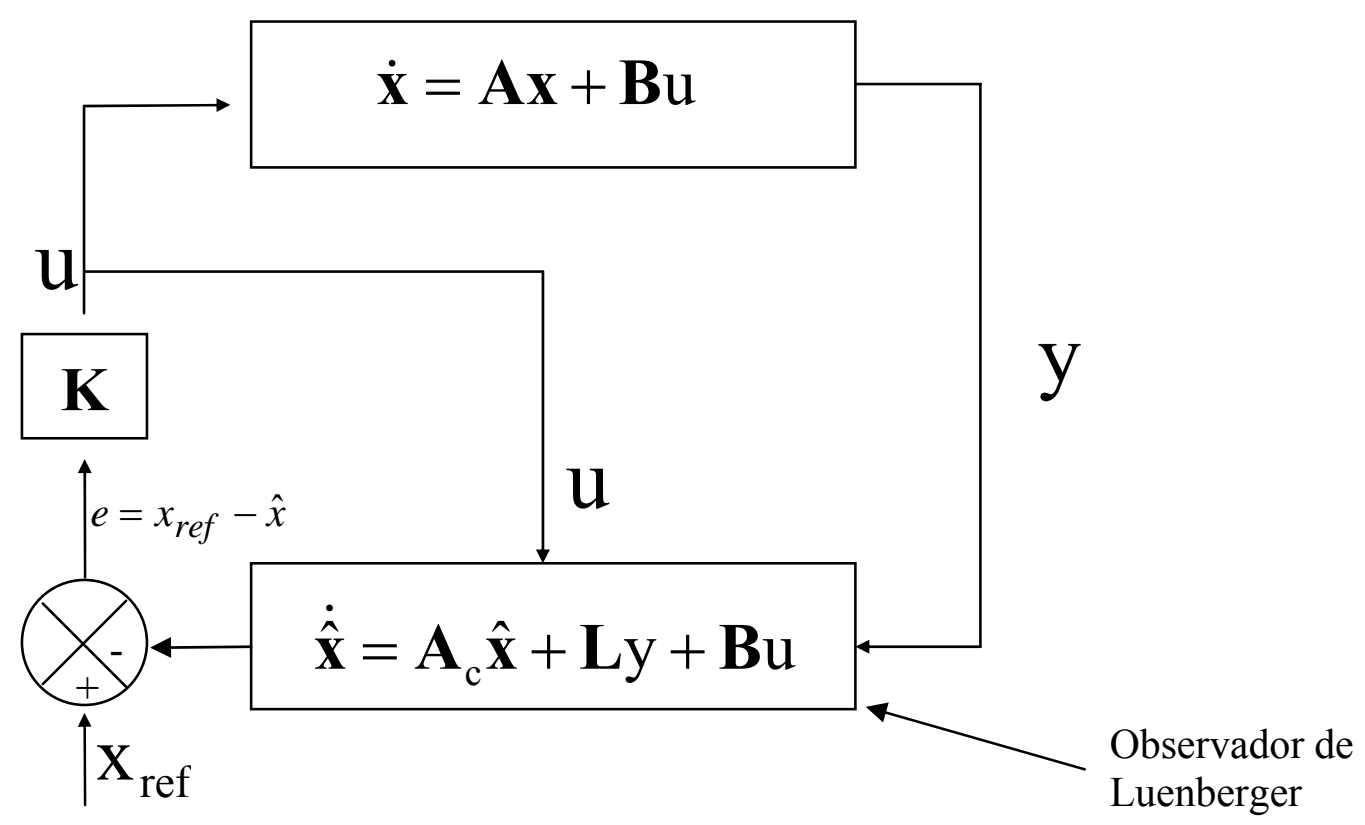

Figura $5.1-\quad$ Sistema de controle - observador linear.

A força $u$ é dada por,

$$
u=-\mathbf{K} \mathbf{x} .
$$

onde $\mathrm{K}$ é a matriz de ganhos.

A matriz L é a matriz de ganhos do observador. Vale notar que, no plano complexo, os pólos do observador devem estar localizados à esquerda dos pólos do sistema para que exista garantia de convergência. Isto pode ser compreendido como o 
observardor tendo um amortecimento maior que o sistema real. Desta forma, o observador responde mais rápido que o sistema real, garantindo a convergência do sistema de controle.

A partir da figura 5.1 encontra-se o seguinte sistema de equações diferenciais

$$
\left\{\begin{array}{c}
\dot{\mathbf{x}} \\
\dot{\hat{\mathbf{x}}}
\end{array}\right\}=\left[\begin{array}{cc}
\mathbf{A} & -\mathbf{B K} \\
\mathbf{L C} & \mathbf{A}_{c}-\mathbf{B K}
\end{array}\right]\left\{\begin{array}{l}
\mathbf{x} \\
\hat{\mathbf{x}}
\end{array}\right\}+\left\{\begin{array}{l}
\mathbf{B} \\
\mathbf{B}
\end{array}\right\} u_{r e f},
$$

onde $\mathbf{A}_{C}=\mathbf{A}-\mathbf{L C}$ e $u_{r e f}$ é um valor arbitrário de força.

A solução do sistema 5.7 leva à dinâmica do sistema de controle. Essa solução é feita através de integração numérica via Runge-Kutta.

Primeiramente, são apresentados resultados do sistema utilizando-se valores de ganhos encontrados por tentativa e erro. Em seguida, apresentam-se soluções utilizando-se as expressões ótimas encontradas na seção anterior.

A matriz $\mathrm{C}$ pode ser determinada de,

$$
\mathrm{y}=\mathbf{h q}
$$

onde $\mathbf{h}$ é o vetor posição dos sensores.

De 5.8 aplicando a matriz dos autovetores $\mathbf{T}$ vem

$$
y=\mathbf{h} \mathbf{T} \mathbf{z} .
$$

A expressão 5.9 pode ser escrita na forma

$$
y=\mathbf{c} \mathbf{z}, \quad \text { onde } \mathbf{c}=\mathbf{h} \mathbf{T} .
$$

Pode-se escrever 5.10 na forma, 
$y=\mathbf{C} \mathbf{x}$,

onde $\mathbf{C}=\left[\begin{array}{ll}\mathbf{0} & \mathbf{c}\end{array}\right]$.

5.11 


\subsection{Riser Reto sem Peso Próprio}

Esta seção estuda os efeitos da introdução de um sistema de controle ativo para o riser reto sem peso próprio do capítulo 3. Para tanto, admite-se uma força modal genérica de atuador $f_{u}$ que é introduzida na equação não-linear do oscilador modal. Neste contexto, não é abordado como este controle será executado fisicamente. Tal controle poderia ser introduzido por atuadores, variações da força normal, variações da vazão interna, strakes e etc., ou uma combinação destes últimos.

Assim, considera-se a seguinte equação não-linear do oscilador do riser reto sem peso próprio retirada do capítulo 3.

$$
\ddot{U}+4,32 \times 10^{-2} U-5,28 \times 10^{-5} U^{3}+6,31 \times 10^{-3} U V^{2}=0
$$

Adiciona-se à equação 5.12 a força modal proveniente do sistema de controle:

$$
\ddot{U}+4,32 \times 10^{-2} U-5,28 \times 10^{-5} U^{3}+6,31 \times 10^{-3} U V^{2}=f u
$$

Nesta seção, a alocação de pólos será feita pela imposição de valores de taxas de amortecimento e pelo aumento da tensão no riser. Através desses valores de amortecimento determinam-se os pólos e, por conseguinte, as matrizes de ganhos do atuador e do observador. Posteriormente, será utilizado o método LQR (Linear Quadratic Regulator) para a alocação de pólos. O aumento da tensão no riser é introduzido pela força de controle modal fu (equação 5.6), que possui duas parcelas uma em $U$ e outa em $V$. A parcela em $U$ influência a rigidez do sistema e, portanto, altera a freqüência do mesmo. É através desta parcela que a alteração da tensão é introduzida.

Para a resposta da figura 5.2 tem-se a taxa de amortecimento $\xi=0.20$. A amplitude inicial adotada é de $10 \mathrm{~m}$. A velocidade modal inicial é zero. Como anteriormente, todos os exemplos terão velocidade inicial modal nula. Em todos os exemplos deste capítulo, o sistema de controle foi aplicado apenas no sistema não- 
linear. Do capítulo 3, sabe-se que o período não-linear para a mesma amplitude modal inicial, vale 29.33s. Com a introdução do sistema de controle, o período muda para 30,08s. Ou seja, o período não-linear tende para o período linear 30,23s e, portanto, existe uma flexibilização do sistema estrutural. Isto acontece, pois o sistema de controle diminui o valor da amplitude do movimento modal ao longo do tempo e, desta forma, a dinâmica se aproxima da dinâmica do sistema linear.

Não obstante, a figura 5.3 mostra o diagrama de fase para a resposta linear e a resposta para o sistema não-linear com o sistema de controle. Observando essa figura é possível notar que o sistema não-linear se distancia da resposta linear seguindo a tendência do diagrama não-linear apresentado na figura 3.4. No entanto, o sistema de controle se faz presente conduzindo o sistema à origem. É interessante que mesmo sob a influência de efeitos não-lineares o sistema de controle é capaz de eliminar as vibrações. As figuras 5.5 e 5.6 reforçam a robustez do sistema de controle mesmo para uma amplitude inicial de $20 \mathrm{~m}$. Nestas figuras fica mais clara a tendência inicial do sistema para o modelo não-linear que é atenuada pelo sistema de controle, que leva o sistema estrutural para a região de comportamento predominantemente linear.

A figura 5.4 mostra a resposta da força modal de controle fu no tempo para a amplitude inicial de $10 \mathrm{~m}$ e a figura 5.7 para amplitude inicial de $20 \mathrm{~m}$. A força modal de controle fu é dada em Newtons. No entanto, ela representa apenas a projeção da força de controle no modo de vibração estudado. Ainda assim, verificou-se, durante os estudos, que seus valores reduzidos são devidos à escolha da tensão no riser como variável de controle, evidenciando, desta forma, a baixa controlabilidade do sistema. Outras formas de controle foram vislumbradas e constam da lista de trabalhos futuros. Uma posível abordagem pode ser apreciada em [91]. Nota-se que a força inicia com valor nulo $\left(f_{u}=0\right.$ para $\left.t=0\right)$. Portanto, existe um intervalo de tempo para que o sistema de controle se torne realmente efetivo. Mesmo com este efeito de atraso, o sistema de controle ativo mostra-se eficiente na dissipação das vibrações e na natural redução das amplitudes modais. Tais propriedades interferem diretamente na vida à fadiga dos risers, tendo, graças à redução da amplitude e do número de ciclos, efeitos positivos. 


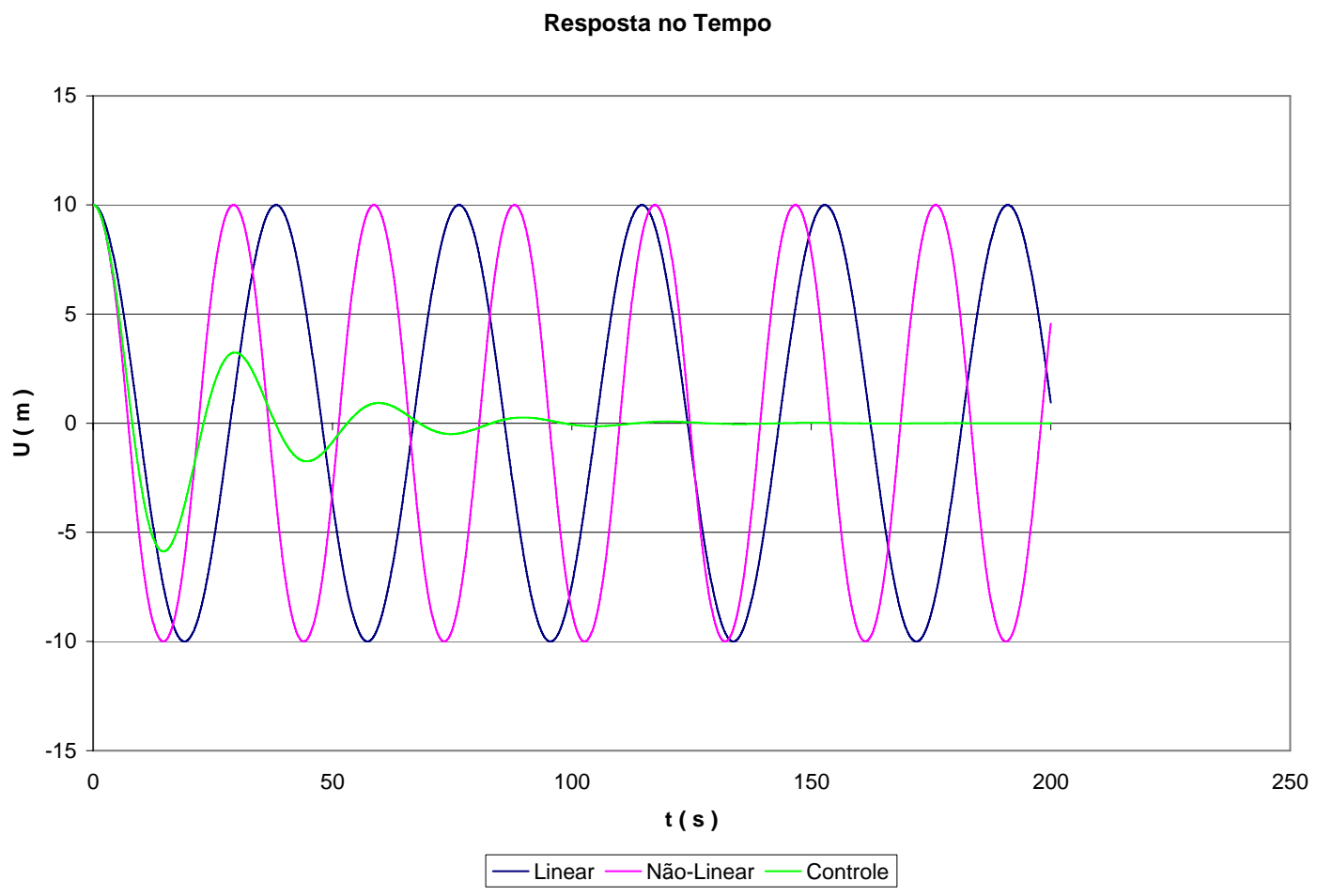

Figura $5.2-\quad$ Riser reto sem peso próprio com controle $\xi=0.20$.

Primeiro modo; amplitude inicial de $10 \mathrm{~m}$.

Períodos: 30,23 s (linear); 29.33 s (Não-Linear); 30,08 s (Controle).

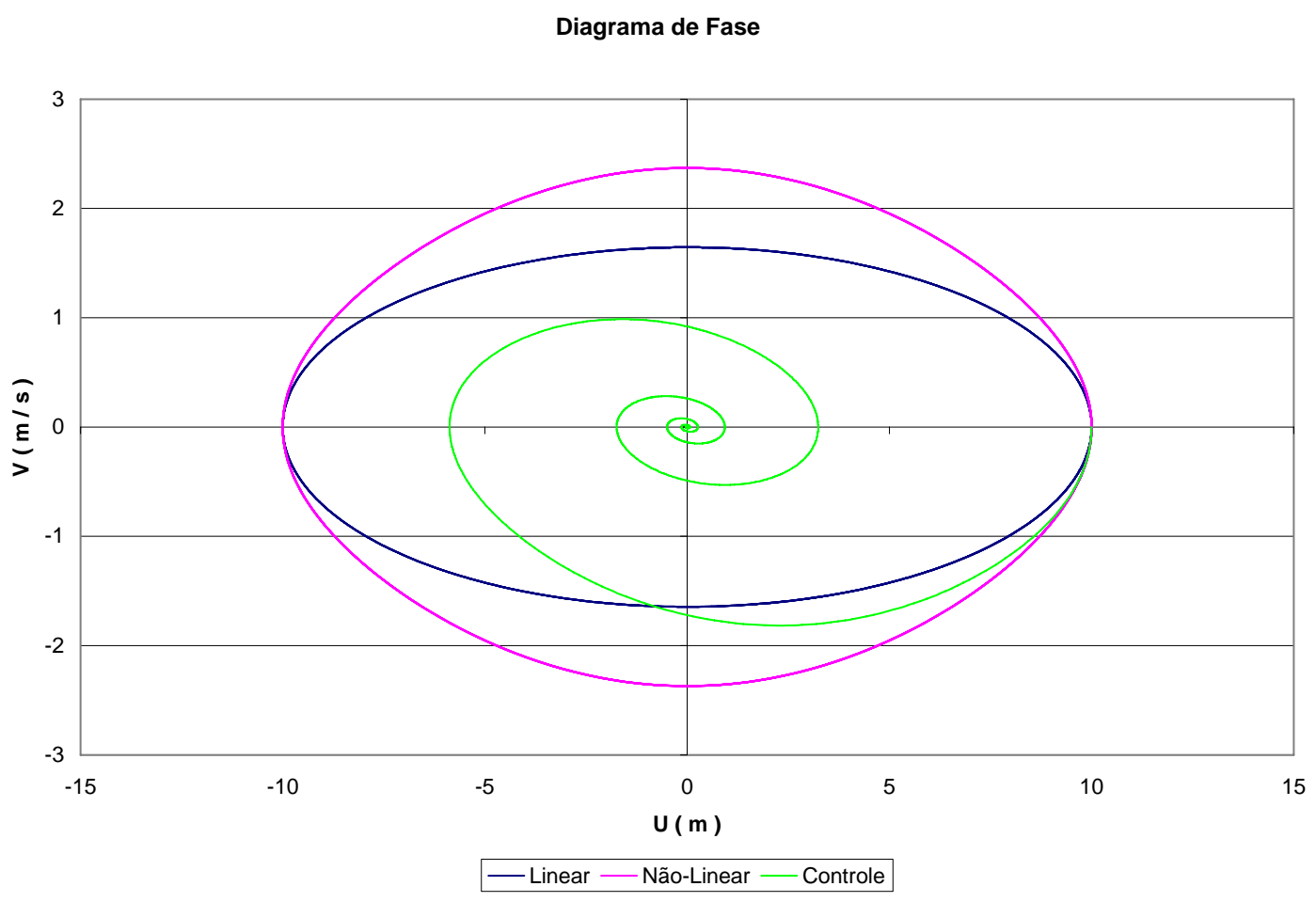

Figura $5.3-\quad$ Diagrama de fase; $\xi=0.20$; primeiro modo; amplitude $10 \mathrm{~m}$ 


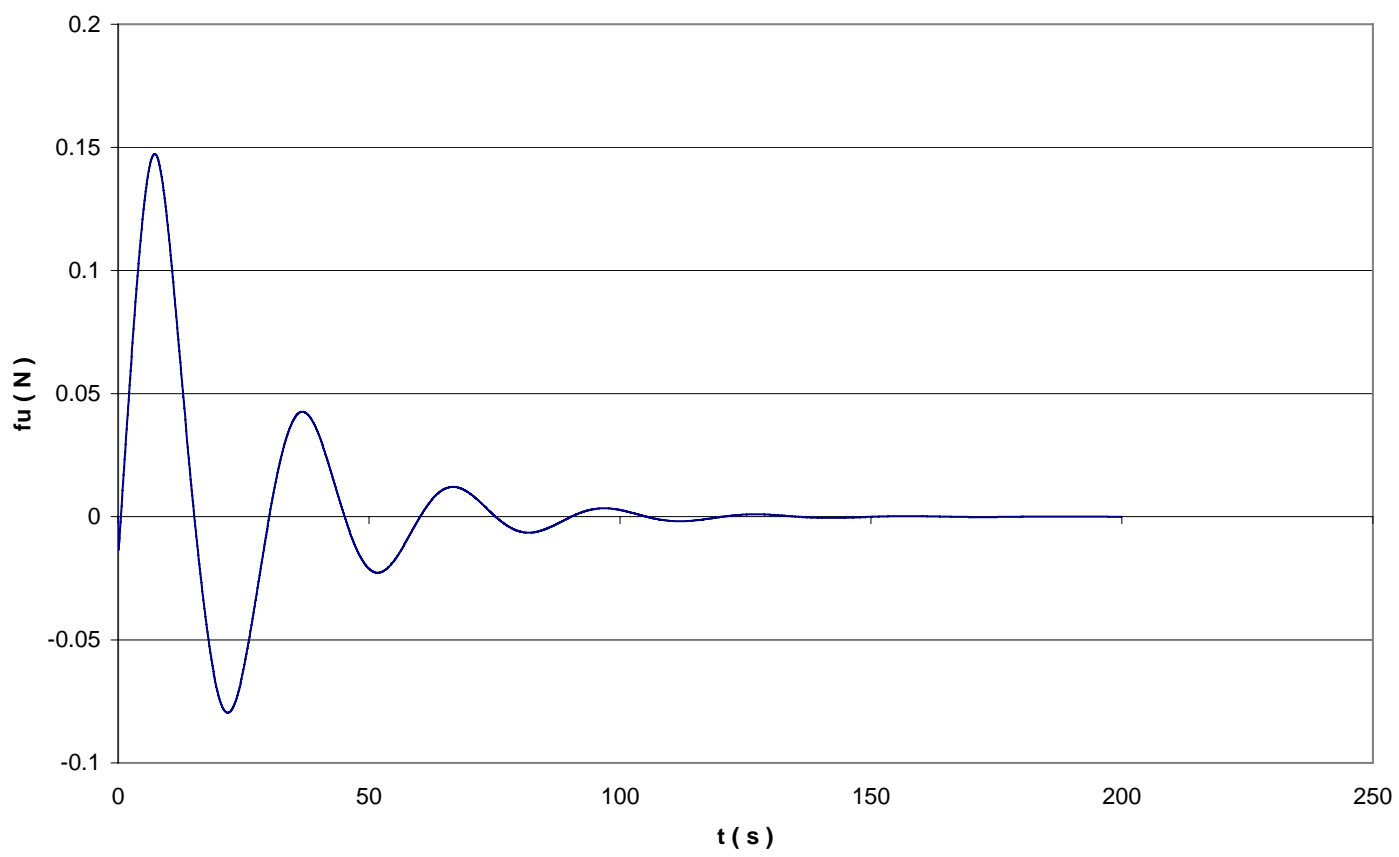

Figura $5.4-$ Força de controle; $\xi=0.20$; primeiro modo; amplitude $10 \mathrm{~m}$

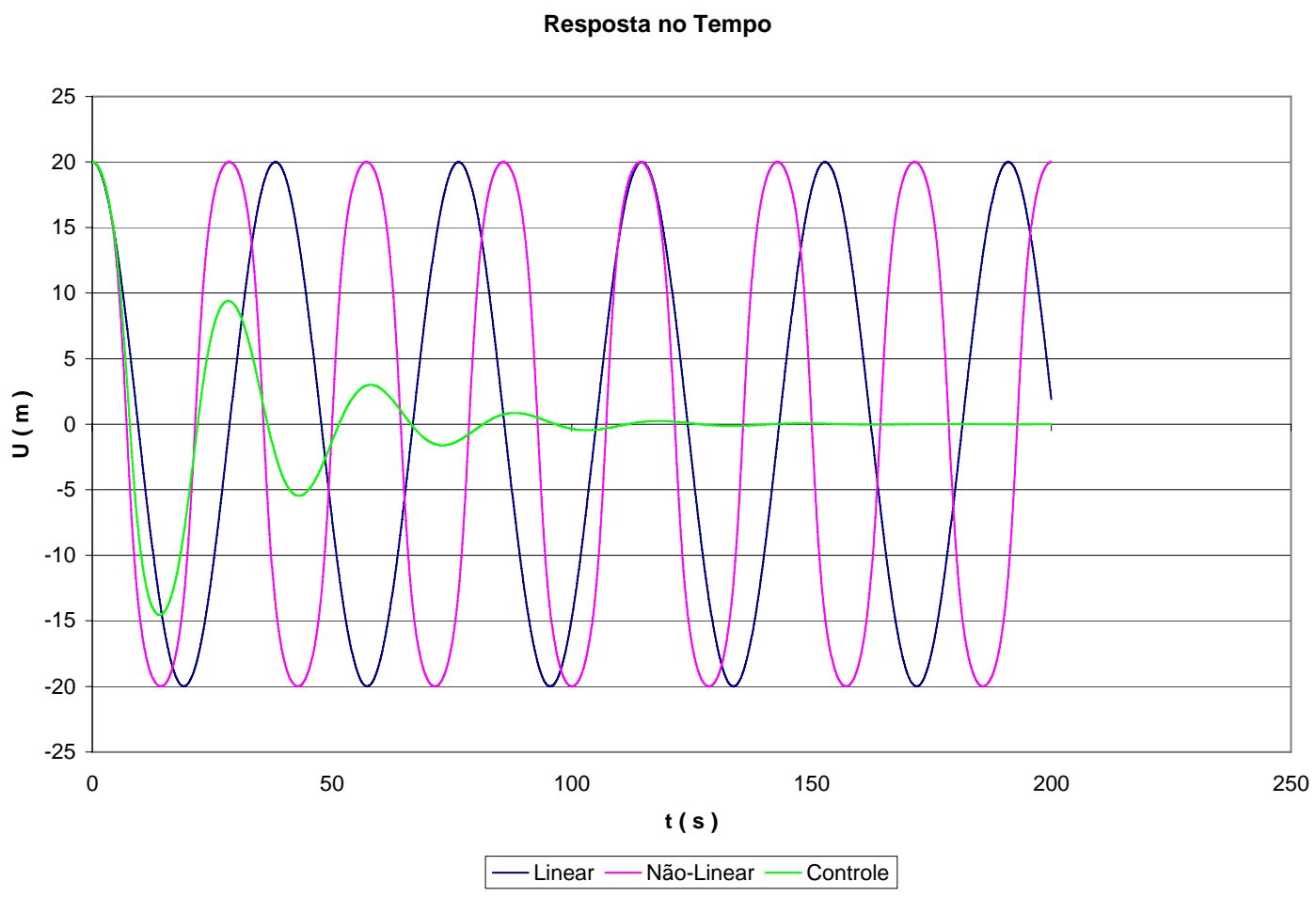

Figura $5.5-\quad$ Riser reto sem peso próprio com controle $\xi=0.20$.

Primeiro modo; amplitude inicial de $20 \mathrm{~m}$.

Períodos: 30,23 s (linear); 29,98 s (MEF). 


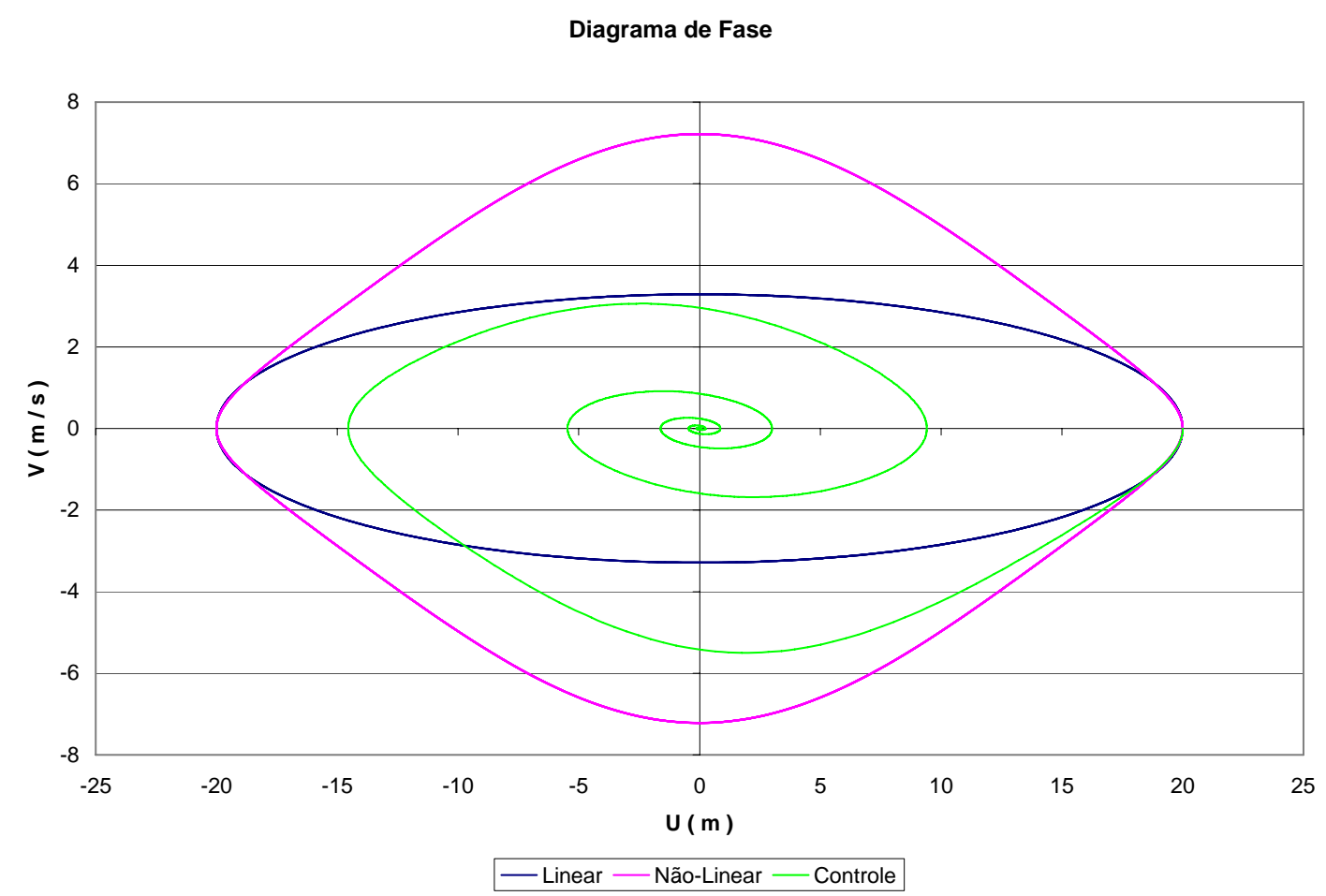

Figura 5.6 - Diagrama de fase; $\xi=0.20$; primeiro modo; amplitude $20 \mathrm{~m}$

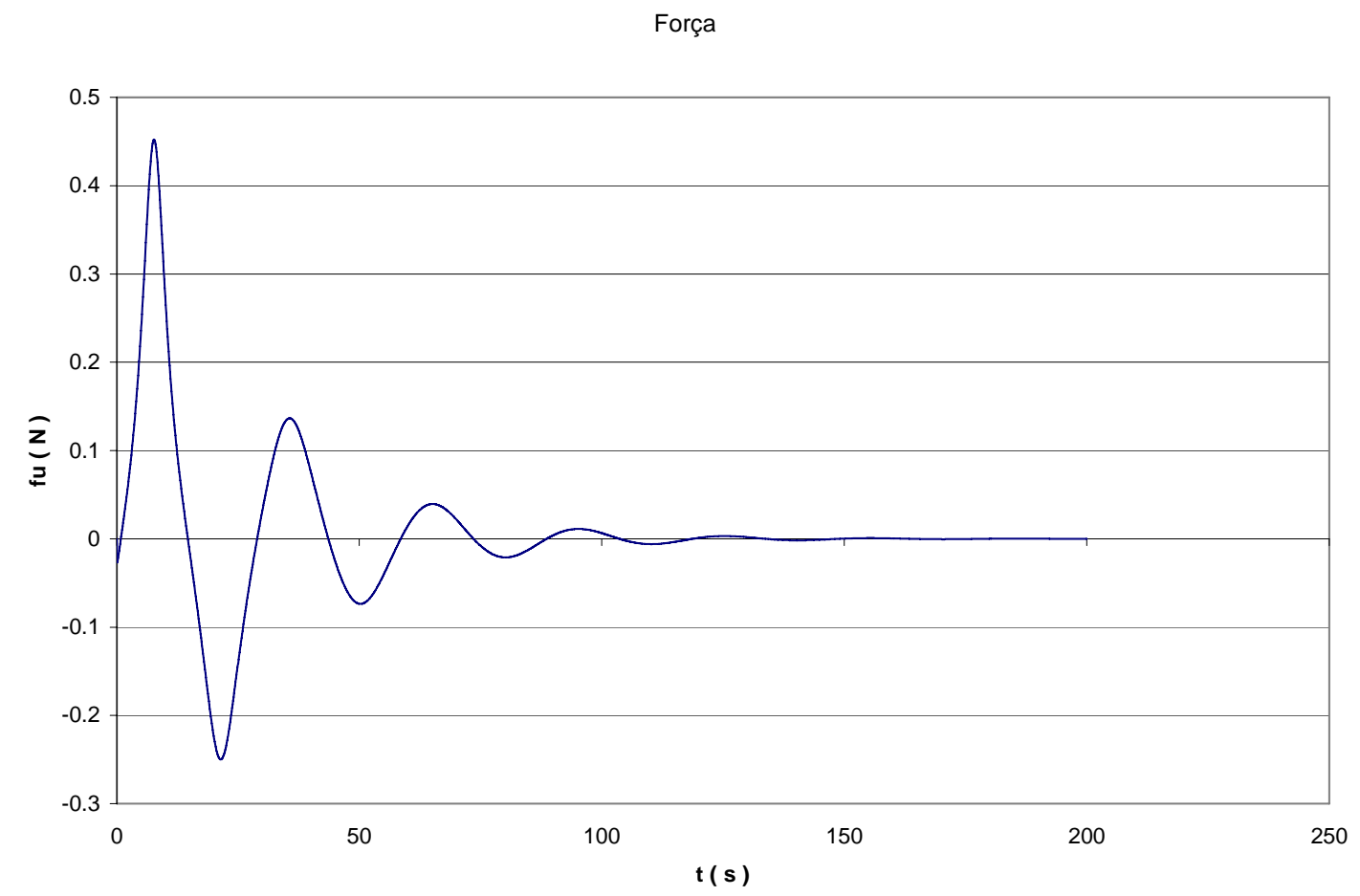

Figura $5.7-$ Força de controle; $\xi=0.20$; primeiro modo; amplitude $20 \mathrm{~m}$. 
Observa-se para esta resposta um aumento da protensão do riser de $\Delta T_{0}=7,88 \mathrm{tf}$ ou um aumento de 3,94\%. A introdução do sistema de controle leva a uma taxa de amortecimento modal de $\zeta=0,20$, como havia sido definido na alocação de pólos. Ou seja, as respostas encontradas nesta seção poderiam ser alcançadas em um modelo físico pelo aumento da protensão em 3,94\% e pela introdução de um sistema de dissipação de energia equivalente a uma taxa de amortecimento $\operatorname{de} \zeta=0,20$ para o primeiro modo de vibração.

Desta forma, torna-se possível determinar valores de protensão adequados a determinadas intensidades de controle. O projeto do sistema de controle e, portanto, da tensão no riser dependem das solicitações e da capacidade da embarcação em suportar esforços extras, que seriam introduzidos para redução das vibrações. Não obstante, o procedimento aqui apresentado auxilia a determinação das protensões e taxas de amortecimento adequadas para um determinado sistema estrutural. 


\subsection{Riser Reto com Peso Próprio}

Considera-se nesta seção o riser reto com peso próprio abordado no capítulo 3 (seção 3.2). Para o estudo do sistema de controle, admite-se o primeiro modo normal não-linear. A equação não-linear do oscilador modal pode ser observada em 5.14, já com a introdução do termo de controle $f_{u}$. Como mencionado na seção 5.1 , não é abordado como este controle será executado fisicamente. A utilização de atuadores, variações da força normal, variações da vazão interna, strakes e etc. são condicionadas às propriedades da embarcação, às condições de carregamento, etc.

$$
\ddot{U}+2,70 \times 10^{-2} U+2,52 \times 10^{-4} U^{3}-2,27 \times 10^{-2} U V^{2}=f u
$$

A alocação de pólos será feita pela imposição de valores de taxas de amortecimento e pelo aumento da tensão no riser. Através desses valores de amortecimento determinam-se os pólos e, por conseguinte, as matrizes de ganhos do atuador e do observador. Para as respostas das figuras 5.8, 5.9 e 5.10 tem-se a taxa de amortecimento $\xi=0.20$. A amplitude inicial adotada é de $10 \mathrm{~m}$. A velocidade modal inicial é zero. Como anteriormente, todos os exemplos terão velocidade modal inicial nula.

Nota-se que o termo em $U V^{2}$ tem coeficiente negativo que leva a uma redução da freqüência do sistema estrutural. Tal redução será influenciada pela força do sistema de controle $f_{u}$. Como pode ser observado na figura 5.10. O período não-linear sem controle vale $39,36 \mathrm{~s}$, enquanto que com a introdução da força $f_{u}$ o período cai para 38,09s aproximando-se do linear 38,21s. Essa alteração pode ser explicada pela redução das amplitudes e velocidades que podem ser apreciada nas figuras 5.8 e 5.9.

Não obstante, o sistema de controle implica um aumento na protensão de $\Delta T_{0}=5,10 t f$ ou 2,55\%. Esse sistema de controle leva, também, a uma taxa de amortecimento de $\zeta=0,20$. Tal alteração da força de protensão influencia a freqüência aumentando-a. Nota-se que a freqüência do sistema com controle é maior do que a do sistema linear. 


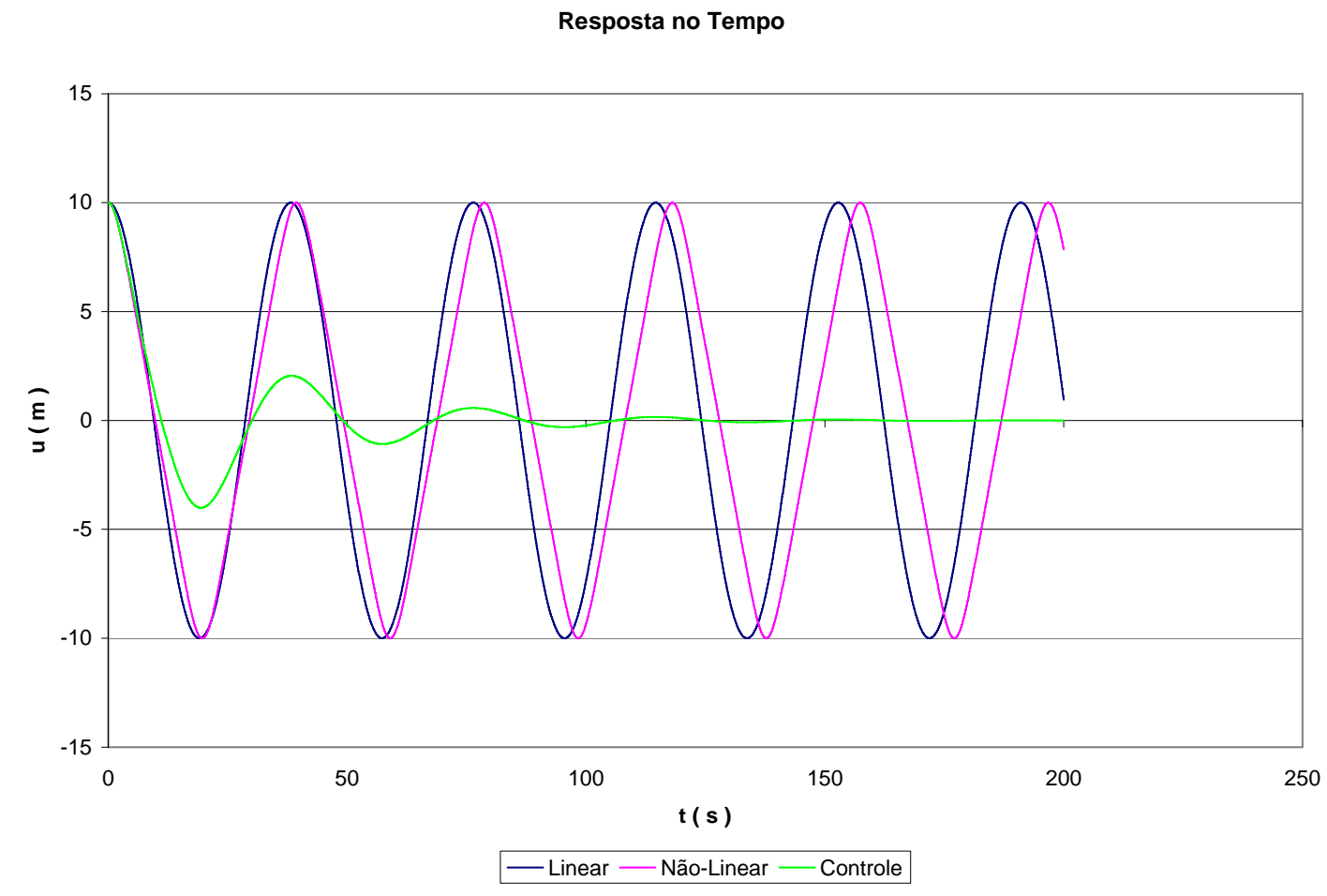

Figura $5.8-\quad$ Riser reto com peso próprio com controle $\xi=0.20$.

Primeiro modo; amplitude inicial de $10 \mathrm{~m}$.

Períodos: 38,21 s (linear); 39.36 s (não-linear); 38,09 s (controle).

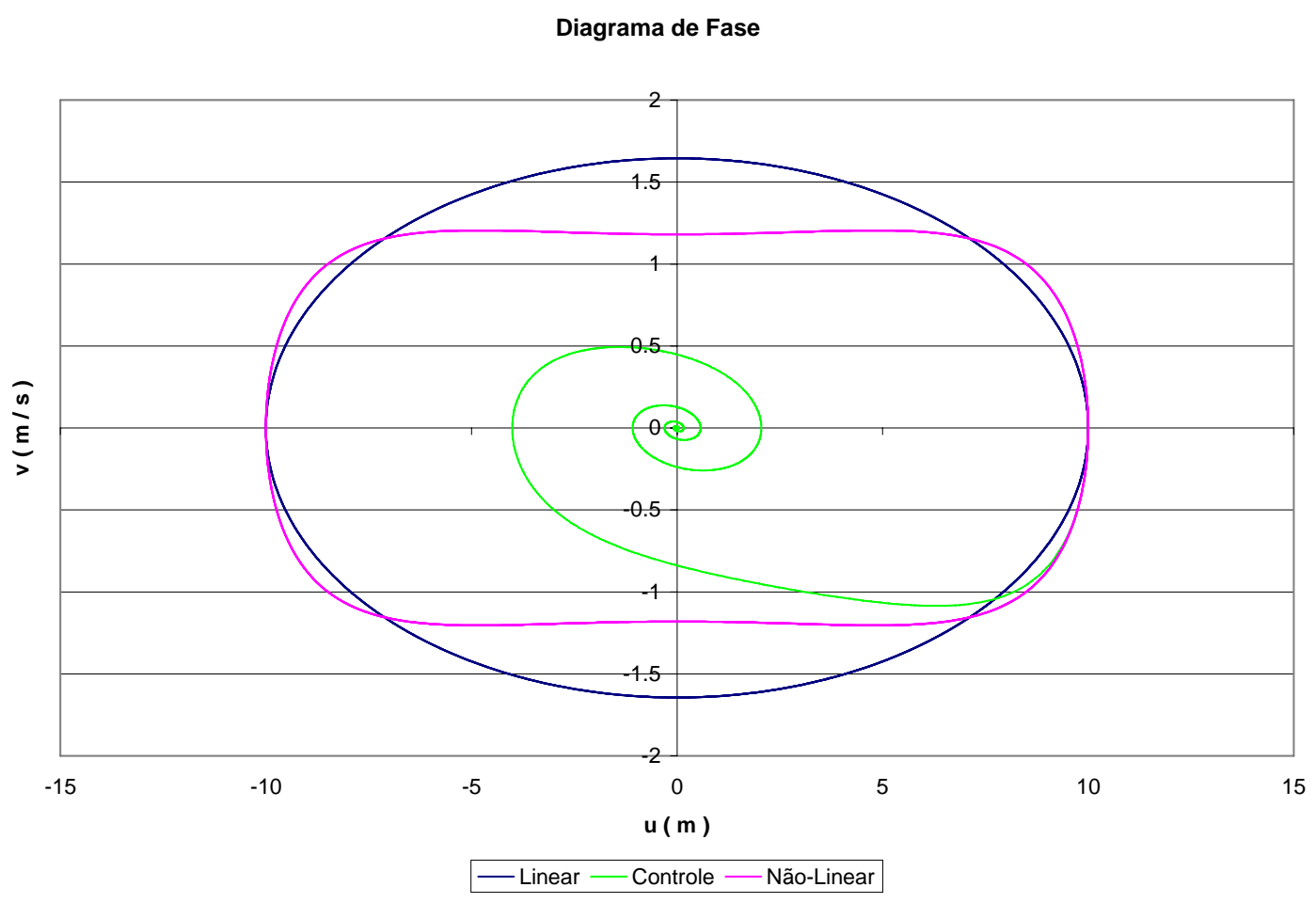

Figura $5.9-\quad$ Diagrama de fase; $\xi=0.20$; primeiro modo; amplitude $10 \mathrm{~m}$ 


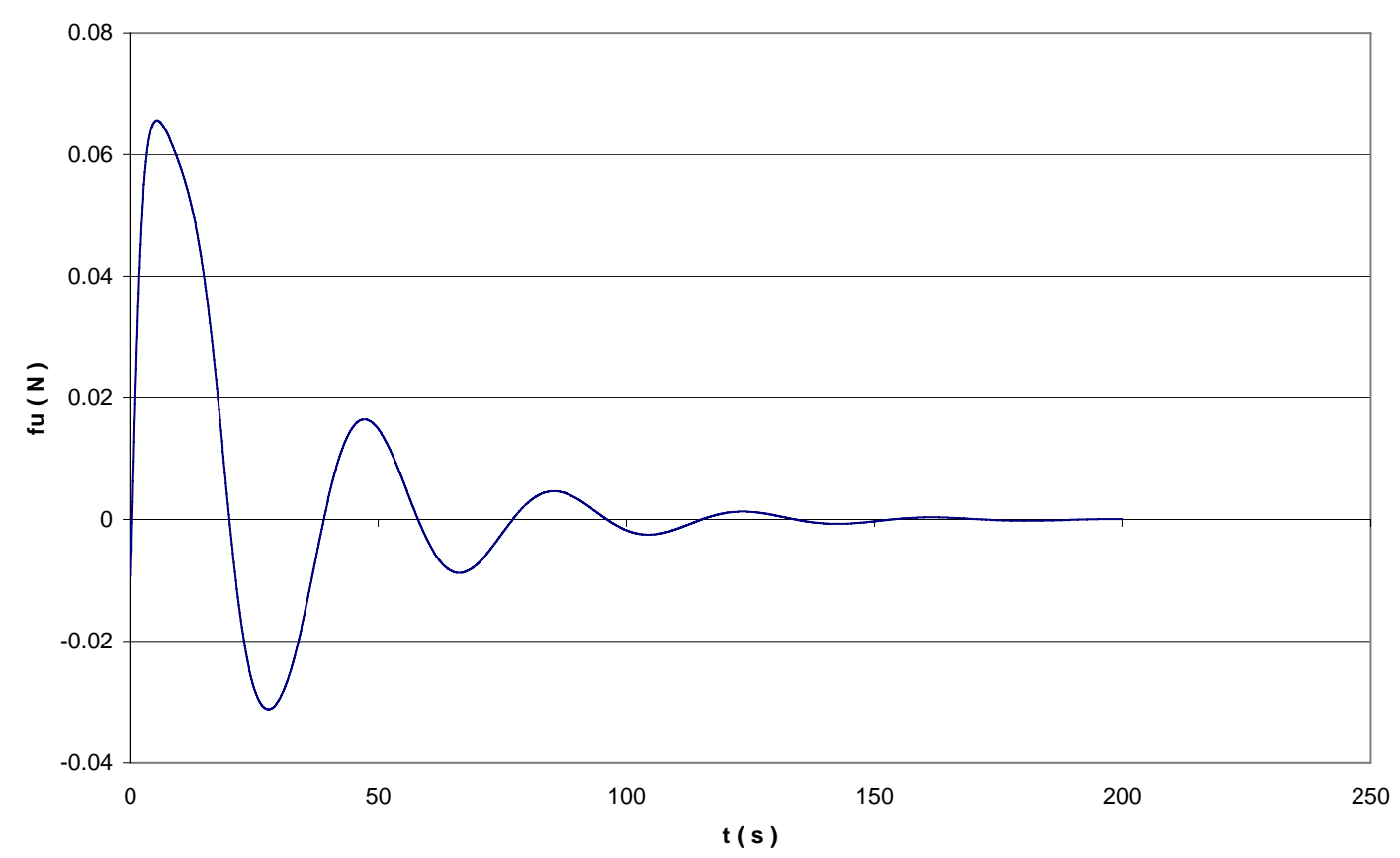

Figura 5.10 - Força de controle; $\xi=0.20$; primeiro modo; amplitude $10 \mathrm{~m}$

Na figura 5.11 o período do sistema com controle $(38,34 \mathrm{~s})$ continua menor do que o período do sistema não-linear $(50,77 \mathrm{~s})$ sem controle. De fato, o período é significativamente menor, o que mostra a importância do sistema de controle para grandes amplitudes. Com relação ao sistema linear (38,21s), o sistema com controle apresenta período pouco maior devido ao aumento da amplitude inicial. O aumento da amplitude inicial supera o efeito do aumento da protensão. Isto significa que, apesar do aumento da rigidez devido ao aumento da protensão inicial, o efeito não-linear oriundo do termo $U V^{2}$ leva a uma leve flexibilização do sistema tornando o período do sistema com controle pouco maior que o período do sistema linear. Em outras palavras, o sstema de controle "lineariza" o sistema original, graças à rápida diminuição das amplitudes.

As figuras 5.11 e 5.12 mostram a robustez do sistema de controle para uma amplitude inicial de $20 \mathrm{~m}$. Nestas figuras fica nítida a tendência inicial do sistema para o modelo não-linear que é atenuada pelo sistema de controle. 


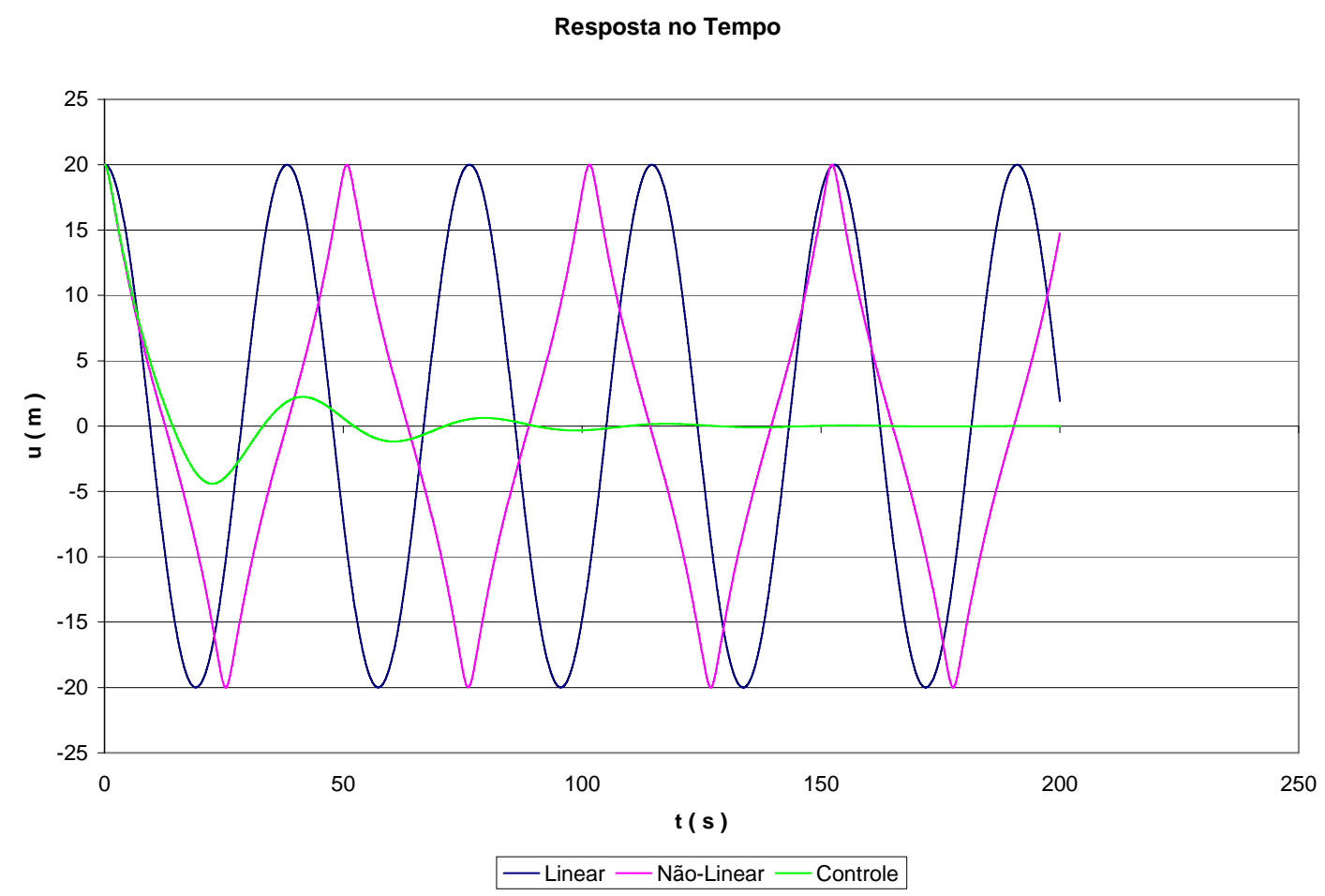

Figura $5.11-$ Riser reto com peso próprio com controle $\xi=0.20$.

Primeiro modo; amplitude inicial de $20 \mathrm{~m}$.

Períodos: 38,21 s (linear); 50.77 (não-linear); 38,34 s (controle).

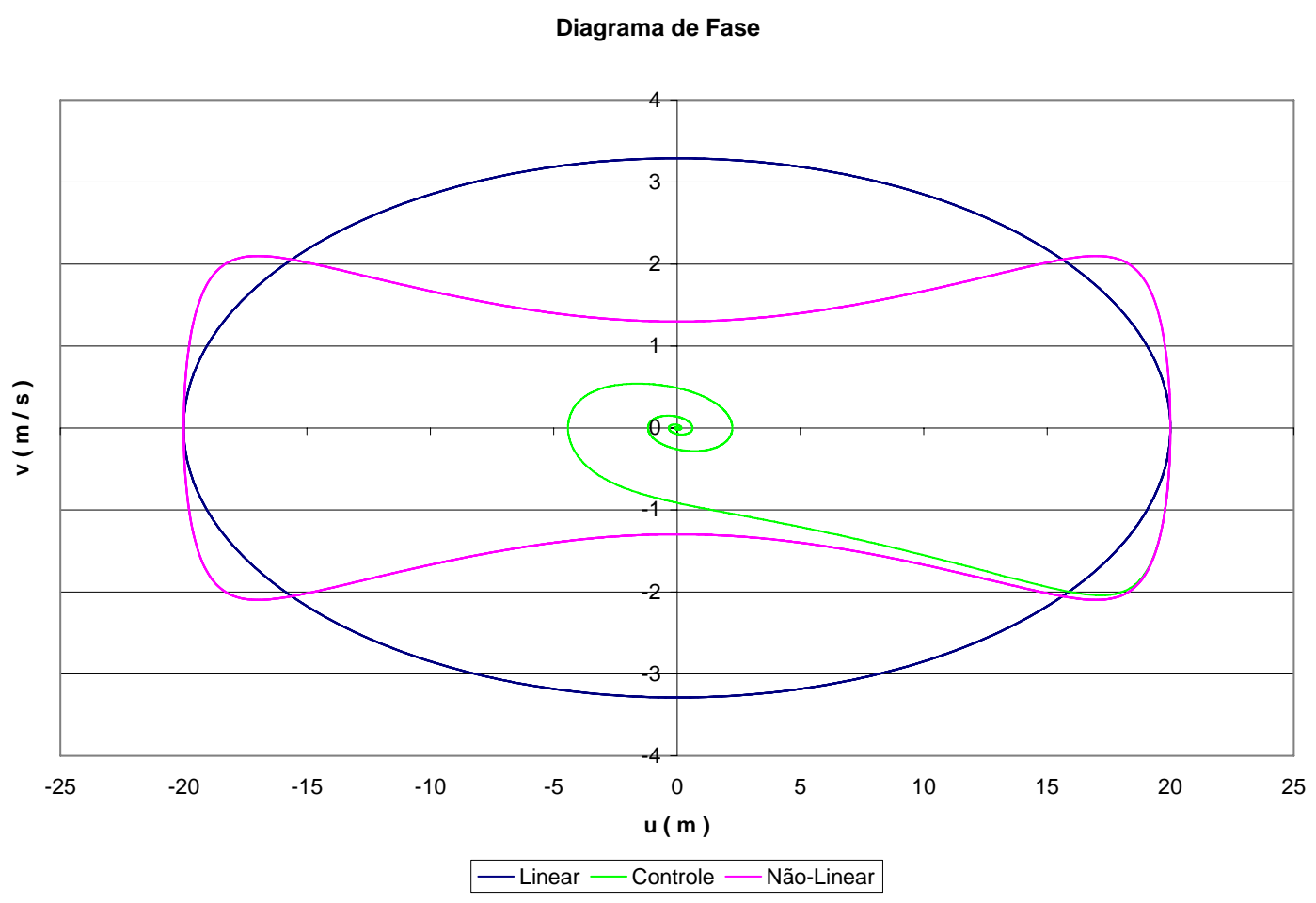

Figura 5.12 - Diagrama de fase; $\xi=0.20$; primeiro modo; amplitude $20 \mathrm{~m}$ 
Força

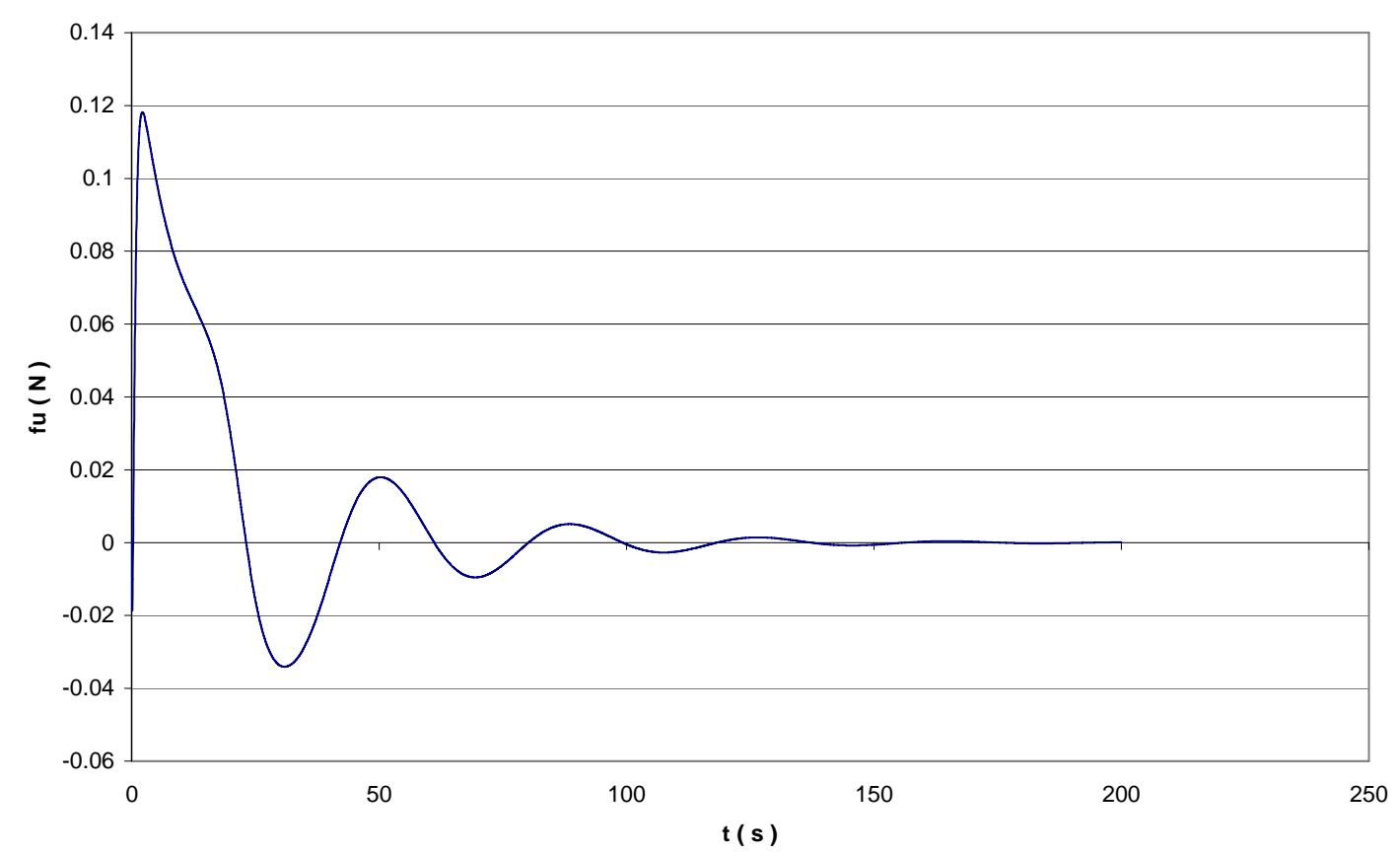

Figura 5.13 - Força de controle; $\xi=0.20$; primeiro modo; amplitude $20 \mathrm{~m}$

A figura 5.13 mostra a resposta da força modal $u$ no tempo para a amplitude inicial de 20m. Novamente, nota-se que a força inicia com valor nulo $\left(f_{u}=0\right.$ para $t=$ 0 ) e decorre um intervalo de tempo para que o sistema de controle se torne realmente efetivo. Mesmo com este efeito de atraso, o sistema de controle ativo continua se mostrando eficiente na dissipação das vibrações. 


\subsection{Riser em Catenária Com Extremos Fixos, VIV e Controle}

Considera-se nesta seção o riser em catenária de extremos fixos e com VIV abordado no capítulo 4 (seção 4.1.1). Para o estudo do sistema de controle admite-se o modo 26 normal não-linear. Como feito na seção 5.1, não é abordado como este controle será executado fisicamente. A utilização de atuadores e outros equipamentos é condicionada às propriedades da embarcação, às condições de carregamento e etc.

Para o riser abordado na seção 4.1.1, estuda-se, nesta seção, seu comportamento dinâmico quando consideradas vibrações induzidas por vórtices (VIV) e controle. O sistema de controle adotado leva a um aumento de $10 \%$ para a taxa de amortecimento e uma elevação da freqüência de 22,5\%. As equações que representam a dinâmica do fluido foram retiradas de Blevins [10] e Parra [67]. Admite-se que, para o seguinte número de Strouhal $S_{t}=\frac{f D}{U} \cong 0,20$, o modo 26 seja excitado, considerando uma velocidade de corrente de $0,5 \mathrm{~m} / \mathrm{s}$. As equações 5.15 e 5.16 representam, respectivamente, as dinâmicas do riser e do fluido.

$$
\begin{gathered}
\ddot{U}+8,1921 U+39 V+22,216 U^{2}-3,0673 V^{2}-70,823 U^{3}+533,54 U V^{2}=38,95 \dot{z}+f u . \\
\dot{z}^{2}+9,8696 z=-4,17 z^{3}+0,3125 \dot{z}+1,98 V .
\end{gathered}
$$

Nas equações 5.15 e 5.16, $U, V$ e fu são, respectivamente, o deslocamento, a velocidades referentes ao grau de liberdade 50 e a força de controle. A variável z é utilizada para representar a dinâmica do fluido.

A figura 5.14 mostra a resposta no tempo para o deslocamento do grau de liberdade 50. Nota-se um regime transiente inicial seguido do regime permanente. No regime permanente, é possível observar uma redução da amplitude total, para o caso nãolinear, de 0,565 $\mathrm{m}$ (seção 4.1.1) para 0,485 $\mathrm{m}$, e, para o caso linear, de 0,567 $\mathrm{m}$ para 0,486 m. Nota-se que na resposta não-linear a amplitude máxima vale 0,2571 $\mathrm{m}$ e a amplitude mínima $-0,2277 \mathrm{~m}$. Tal comportamento está relacionado à assimetria geométrica do sistema estrutural. A resposta linear apresenta extremos iguais a 
$+0,2432 m e-0,2432 m$, no regime permanente. No caso não-linear, a redução da amplitude encontrada é de 14,16\%, em contrapartida, o aumeto necessário para elevar a freqüência em 22,5\% é da ordem de 50\% na tensão de topo do riser. Questiona-se, portanto, a contrabilidade do sistema quando se adota a tensão de topo do riser como variável de controle. Observa-se que as forças modais de controle são pequenas frente aos acréscimos de tensão introduzidos no topo do riser, o que evidencia a baixa controlabilidade do sistema. Uma abordagem futura seria a introdução de massas que se deslocassem ao longo do riser, utilizando, por exemplo, robôs, para a atenuação das vibrações. Um ponto de partida poderia ser o trabalho desenvolvido por Terceiro [91].

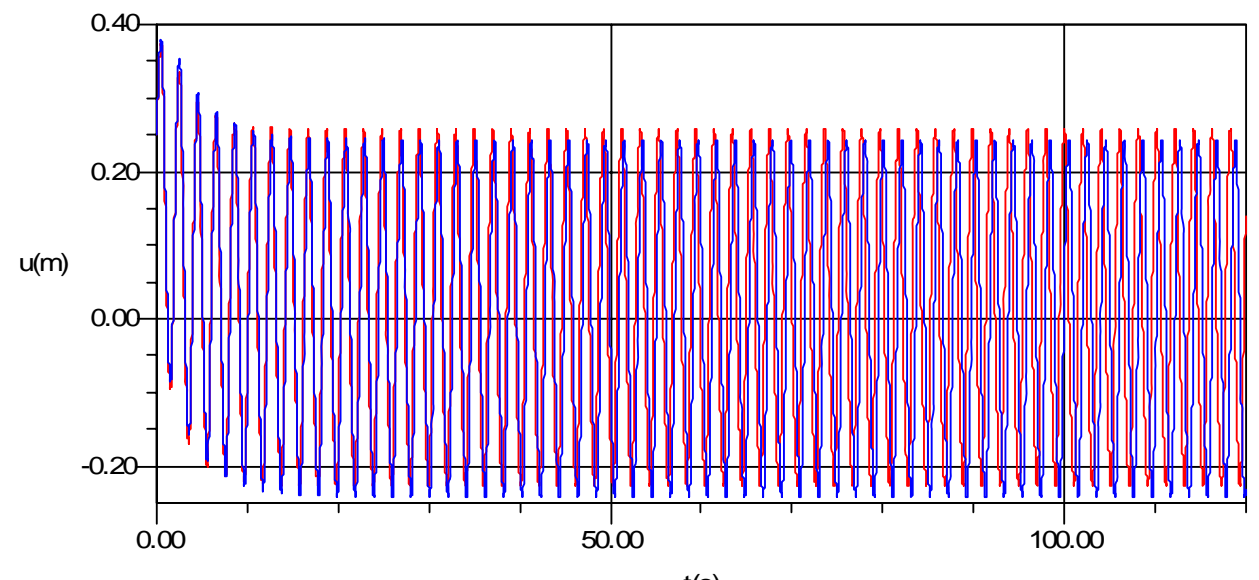

$0.00<\mathrm{X}<1.20 \mathrm{e}+2 ;-0.24<\mathrm{Y}<0.38$

$t(s)$

Figura 5.14 - Resposta no tempo - Linear (Azul) e Não Linear (Vermelho).

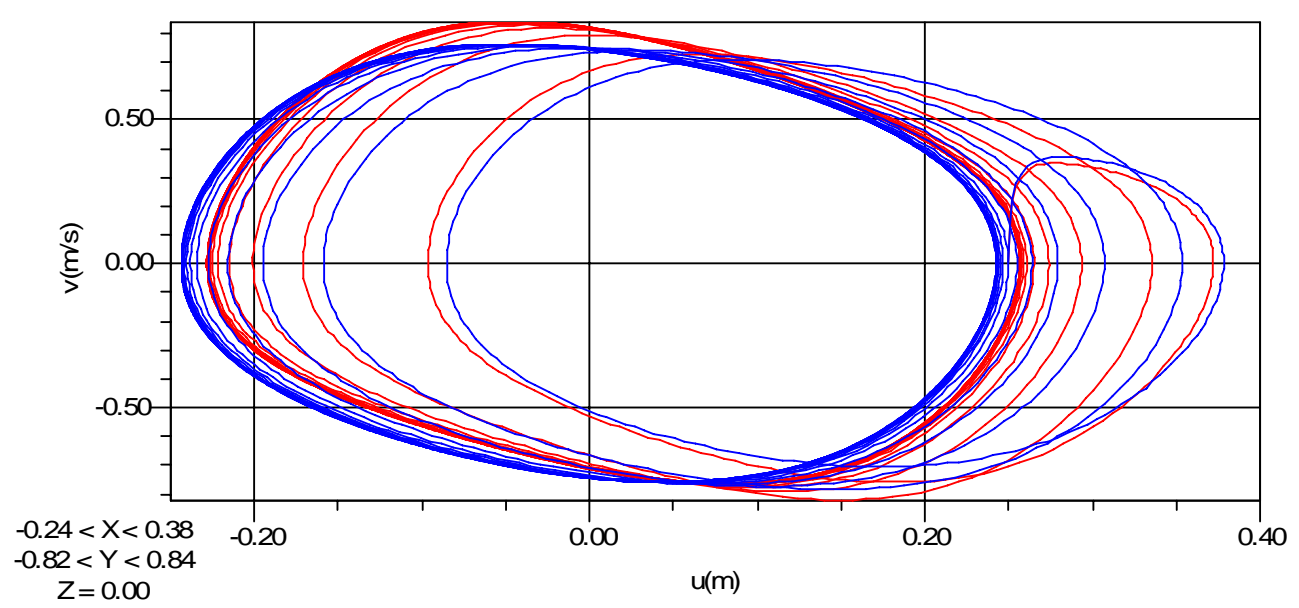

Figura 5.15 - Diagrama de Fase - Linear (Azul) e Não Linear (Vermelho). 
Estes resultados, sobre a dinâmica de risers excitados por VIV, são preliminares, sendo possível e desejável desenvolvimentos futuros. 


\subsection{Controle Ótimo - Regulador Quadrático Linear}

Considere-se o sistema linear:

$$
\dot{\mathbf{x}}=\mathbf{A} \mathbf{x}+\mathbf{B} \mathbf{u},
$$

onde a matriz A não é necessariamente estável (ver [73]) mas o par A, B é controlável. Um sistema dinâmico descrito na forma de espaço de estados é dito controlável se a matrix $C_{b}=\left[\begin{array}{llll}B & A B & \cdots & A^{n-1} B\end{array}\right]$ tem posto $n$, onde $n$ é a ordem da matriz de estados.

Admite-se a função de controle

$$
\mathbf{u}=-\mathbf{K} \mathbf{x}
$$

onde $\mathbf{K}$ é a matriz de ganhos.

Procura-se a matriz $\mathbf{K}$ de tal forma que o funcional

$$
J=\int_{0}^{\infty}\left(\frac{1}{2} \mathbf{x}^{T} \mathbf{Q} \mathbf{x}+\frac{1}{2} \mathbf{u}^{T} \mathbf{R} \mathbf{u}\right) d t
$$

seja mínimo. Na expressão 5.19, Q é positiva semidefinida e $\mathbf{R}$ é estritamente positiva-definida. $\mathrm{O}$ fato de a matriz $\mathbf{R}$ ser estritamente positiva-definida é conseqüência de todo controle ter um custo. A matriz $\mathbf{Q}$ é positiva semidefinida, porque alguns modos de vibração podem ser irrelevantes dependendo do sistema a ser estudado. Nos exemplos que se seguem a calibração de $\mathbf{Q}$ e $\mathbf{R}$ é feita em função de cada sistema a ser controlado.

O problema de otimização é: 


$$
\text { Min } \quad J=\int_{0}^{\infty}\left(\frac{1}{2} \mathbf{x}^{T} \mathbf{Q} \mathbf{x}+\frac{1}{2} \mathbf{u}^{T} \mathbf{R} \mathbf{u}\right) d t
$$

tal que

$$
\dot{\mathbf{x}}=\mathbf{A} \mathbf{x}+\mathbf{B} \mathbf{u}
$$

Utilizando o método dos multiplicadores de Lagrange vem,

$$
\text { Min } \quad J=\int_{0}^{\infty}\left[\frac{1}{2} \mathbf{x}^{\mathrm{T}} \mathbf{Q} \mathbf{x}+\frac{1}{2} \mathbf{u}^{\mathrm{T}} \mathbf{R} \mathbf{u}+\boldsymbol{\lambda}^{\mathrm{T}}(\mathbf{A} \mathbf{x}+\mathbf{B} \mathbf{u}-\dot{\mathbf{x}})\right] \mathrm{dt}
$$

Integrando-se o último termo da expressão 5.21 por partes tem-se,

Min $\quad \mathrm{J}=-\left[\lambda^{\mathrm{T}} \mathbf{x}\right]_{0}^{\infty}+\int_{0}^{\infty}\left(\frac{1}{2} \mathbf{x}^{\mathrm{T}} \mathbf{Q} \mathbf{x}+\frac{1}{2} \mathbf{u}^{\mathrm{T}} \mathbf{R} \mathbf{u}+\lambda^{\mathrm{T}} \mathbf{A} \mathbf{x}+\lambda^{\mathrm{T}} \mathbf{B} \mathbf{u}+\dot{\boldsymbol{\lambda}}^{\mathrm{T}} \mathbf{x}\right) \mathrm{dt}$

Expressando a variação do funcional 5.22, vem

$$
\delta J=\int_{0}^{\infty}\left(\delta \mathbf{x}^{T} \mathbf{Q} \mathbf{x}+\delta \mathbf{u}^{T} \mathbf{R} \mathbf{u}+\boldsymbol{\lambda}^{T} \mathbf{A} \delta \mathbf{x}+\lambda^{T} \mathbf{B} \delta \mathbf{u}+\dot{\lambda}^{T} \delta \mathbf{x}\right) d t
$$

ou, ainda,

$$
\delta J=\int_{0}^{\infty}\left(\delta \mathbf{x}^{T} \mathbf{Q} \mathbf{x}+\delta \mathbf{u}^{T} \mathbf{R} \mathbf{u}+\delta \mathbf{x}^{T} \mathbf{A}^{T} \lambda+\delta \mathbf{u}^{T} \mathbf{B}^{T} \lambda+\delta \mathbf{x}^{T} \dot{\lambda}\right) d t .
$$

Agrupando os termos em $\delta \mathbf{x}^{T}$ e $\delta \mathbf{u}^{T}$, tem-se

$$
\delta J=\int_{0}^{\infty}\left[\delta \mathbf{x}^{T}\left(\mathbf{Q} \mathbf{x}+\mathbf{A}^{T} \boldsymbol{\lambda}+\dot{\lambda}\right)+\delta \mathbf{u}^{T}\left(\mathbf{R} \mathbf{u}+\mathbf{B}^{T} \boldsymbol{\lambda}\right)\right] d t .
$$

A condição de optimalidade é: 


$$
\delta \mathbf{J}=\int_{0}^{\infty}\left[\delta \mathbf{x}^{\mathrm{T}}\left(\mathbf{Q} \mathbf{x}+\mathbf{A}^{\mathrm{T}} \boldsymbol{\lambda}+\dot{\boldsymbol{\lambda}}\right)+\delta \mathbf{u}^{\mathrm{T}}\left(\mathbf{R} \mathbf{u}+\mathbf{B}^{\mathrm{T}} \boldsymbol{\lambda}\right)\right] \mathrm{dt}=0
$$

Da expressão 5.26, conclui-se que

$$
\mathbf{Q} \mathbf{x}+\mathbf{A}^{T} \lambda+\dot{\lambda}=\mathbf{0}
$$

$$
\mathbf{R} \mathbf{u}+\mathbf{B}^{T} \boldsymbol{\lambda}=\mathbf{0} .
$$

Manipulando-se a equação 5.28, encontra-se

$$
\mathbf{u}=-\mathbf{R}^{-1} \mathbf{B}^{\mathrm{T}} \boldsymbol{\lambda}
$$

Para que o controle seja linear, admite-se que

$$
\lambda=\mathbf{P} \mathbf{x} .
$$

Substituindo 5.30 em 5.29, vem

$$
\mathbf{u}=-\mathbf{R}^{-1} \mathbf{B}^{T} \mathbf{P} \mathbf{x} .
$$

Comparando-se 5.18 com 5.31, obtém-se

$$
\mathbf{K}=\mathbf{R}^{-1} \mathbf{B}^{T} \mathbf{P} \text {. }
$$

Substituindo-se 5.17, 5.18, 5.30 e 5.32 em 5.27 tem-se,

$$
\mathbf{Q} \mathbf{x}+\mathbf{A}^{\mathrm{T}} \mathbf{P} \mathbf{x}+\mathbf{P}\left(\mathbf{A} \mathbf{x}-\mathbf{B R}^{-1} \mathbf{B}^{\mathrm{T}} \mathbf{P} \mathbf{x}\right)=\mathbf{0} .
$$

A partir da expressão 5.33 encontra-se a equação matricial de Riccati: 


$$
\mathbf{Q}+\mathbf{A}^{\mathrm{T}} \mathbf{P}+\mathbf{P A}-\mathbf{P B R}^{-1} \mathbf{B}^{\mathrm{T}} \mathbf{P}=\mathbf{0} .
$$

A matriz P é simétrica e positiva-definida, ver [65] e [73]. Essa matriz é encontrada a partir da solução da equação de Riccati. A existência e a unicidade da solução são garantidas se o par $(\mathbf{A}, \mathbf{B})$ for controlável.

\subsection{1 "Riser" Reto sem Peso Próprio}

Esta seção estuda os efeitos da introdução de um sistema de controle ativo ótimo para o riser reto sem peso próprio do capítulo 3. Para tanto, admite-se uma $Q=0,0005 I, Q_{o b}=0,0006 I$ e $R=I$, onde I é a matriz identidade. Estes valores foram calibrados depois de várias tentativas, de forma a encontrar uma resposta adequada. A força modal de atuador genérica $f_{u}$ é introduzida na equação não-linear do oscilador modal, conforme 5.35. Neste contexto, não é abordado como este controle será executado fisicamente.

$$
\ddot{U}+4,32 \times 10^{-2} U-5,28 \times 10^{-5} U^{3}+6,31 \times 10^{-3} U V^{2}=f u
$$

Não obstante, este sistema de controle ótimo representa um aumento na protensão de $\Delta T_{0}=25,04 t f$ ou $12,52 \%$. Esse sistema de controle leva, também, a uma taxa de amortecimento de $\zeta=0,2567$. Tal alteração da força de protensão influencia a freqüência aumentando-a. Nota-se que a freqüência do sistema com controle $(0.03427 \mathrm{~Hz})$ é maior do que a do sistema linear $(0,03308 \mathrm{~Hz})$, conforme figura 5.14. O mesmo sistema estrutural, porém com diferente sistema de controle, apresentado na seção 5.1 leva a uma freqüência ligeiramente inferior $(0,03324 \mathrm{~Hz})$. No entanto, esta variação da frequiência está associada ao aumento na tensão inicial que leva a uma redução das amplitudes. Comparando-se as figuras 5.1 e 5.16, encontram-se as primeiras amplitudes negativas 5,88m e 5,12m, respectivamente. Isto significa uma redução de 12,93\% no valor da primeira amplitude negativa, o que é benéfico para vida à fadiga do riser. 


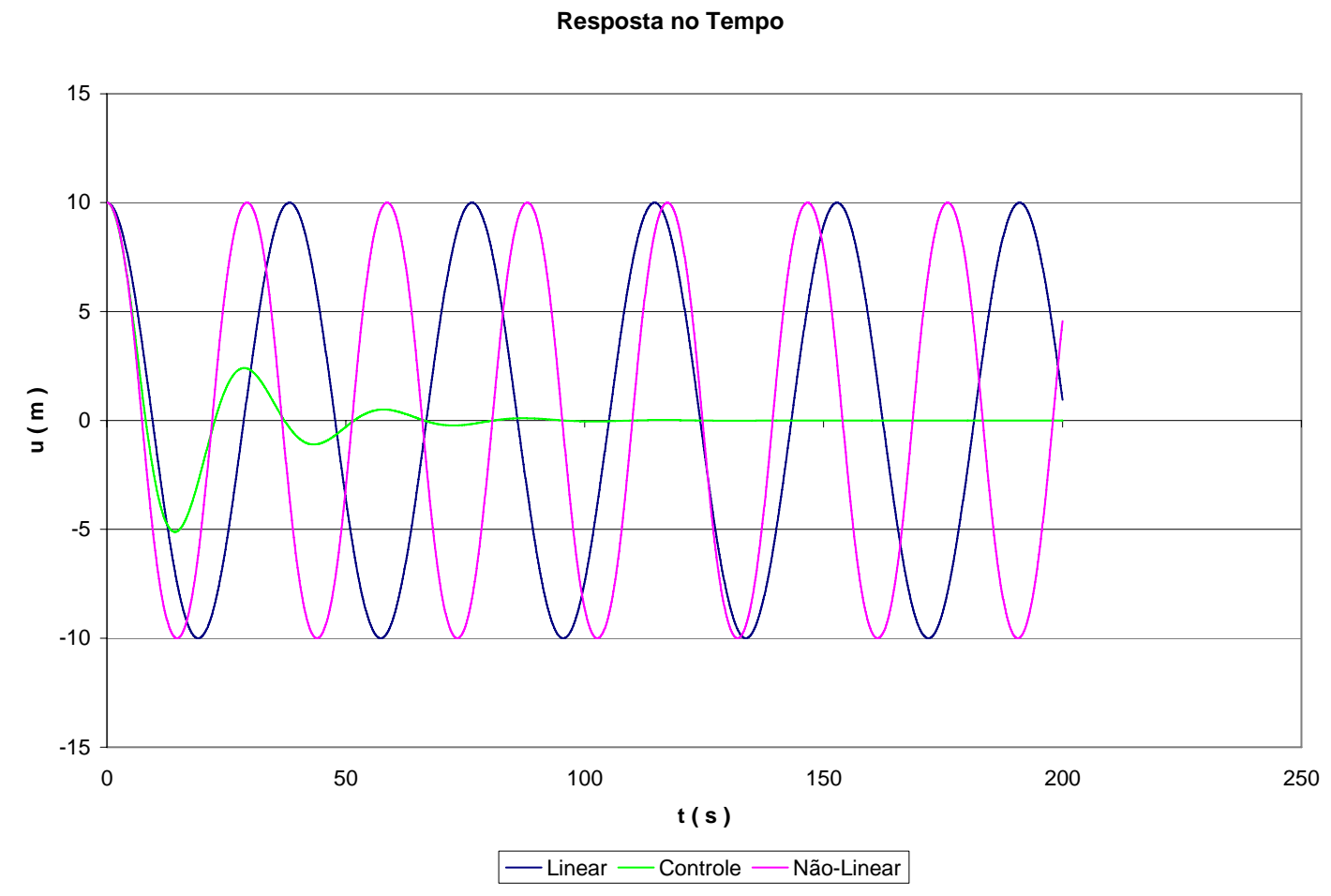

Figura 5.16 - Riser reto sem peso próprio com controle ótimo $Q=0.0005 I \mathrm{e}$ $Q_{o b}=0.0006 I$.

Primeiro modo; amplitude inicial de $10 \mathrm{~m}$.

Períodos: 30,23 s (linear); 29,33 s (não-linear); 29,18 s (controle).

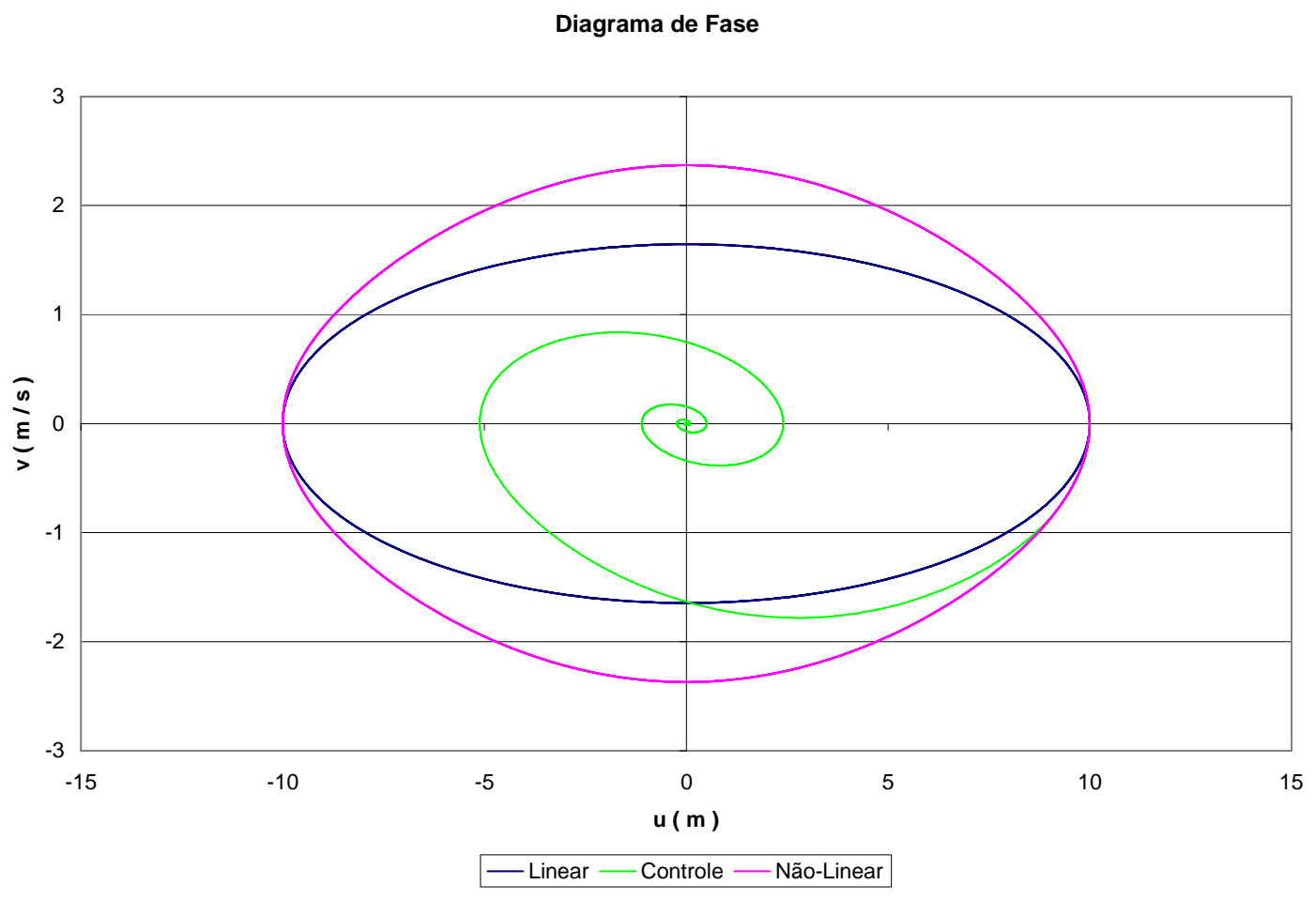

Figura 5.17 - Diagrama de fase; primeiro modo; amplitude inicial de $10 \mathrm{~m}$. 
Força

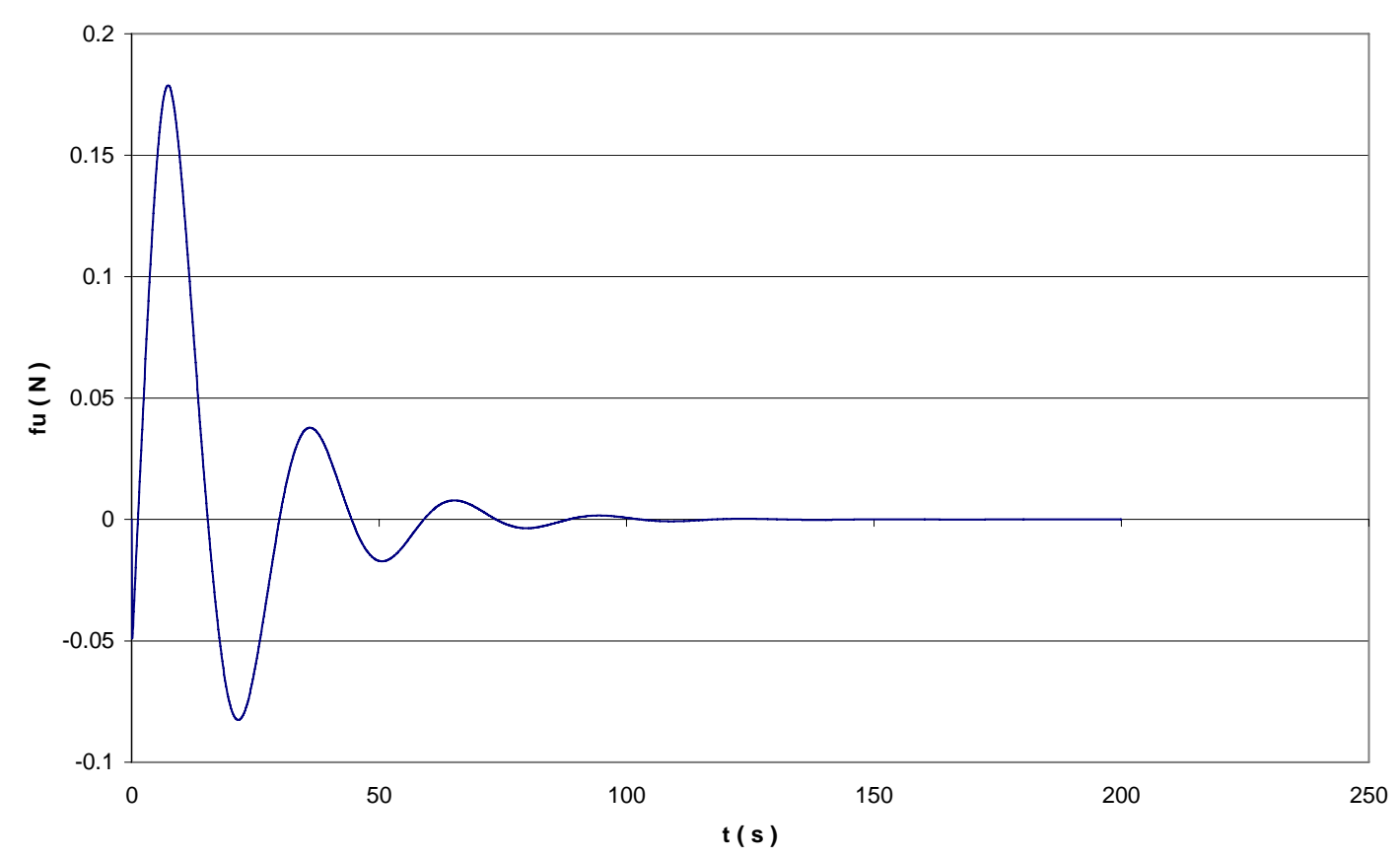

Figura 5.18 - Força de controle; primeiro modo; amplitude inicial de $10 \mathrm{~m}$.

A figura 5.18 mostra a resposta da força modal $u$ no tempo para a amplitude inicial de $10 \mathrm{~m}$ e a figura 5.20 para amplitude inicial de $20 \mathrm{~m}$. A força inicia com valor nulo $\left(f_{u}=0\right.$ para $\left.t=0\right)$. O intervalo de tempo para que o sistema de controle se torne realmente efetivo não impede que o sistema de controle estabilize a dinâmica. Isso se deve ao fato de a força de controle ser uma função não só do deslocamento, mas, também, da velocidade. Observando as figuras 5.17 e 5.20, nota-se que a força de controle atua mesmo nos trechos de deslocamentos pequenos, uma vez que nesses intervalos as velocidades são significativas.

Inicialmente o sistema com controle tende a seguir o sistema não-linear. É possível notar esse comportamento na figura 5.16, porém a tendência é ainda mais evidente na figura 5.19. Nota-se, também, que o sistema de controle ótimo mantém-se estável. Comparando-se as figuras 5.5 e 5.19, é possível observar as primeiras amplitudes negativas $-14,55 m$ e $-13,43 m$, respectivamente. Isto significa uma redução de $7,70 \%$. 


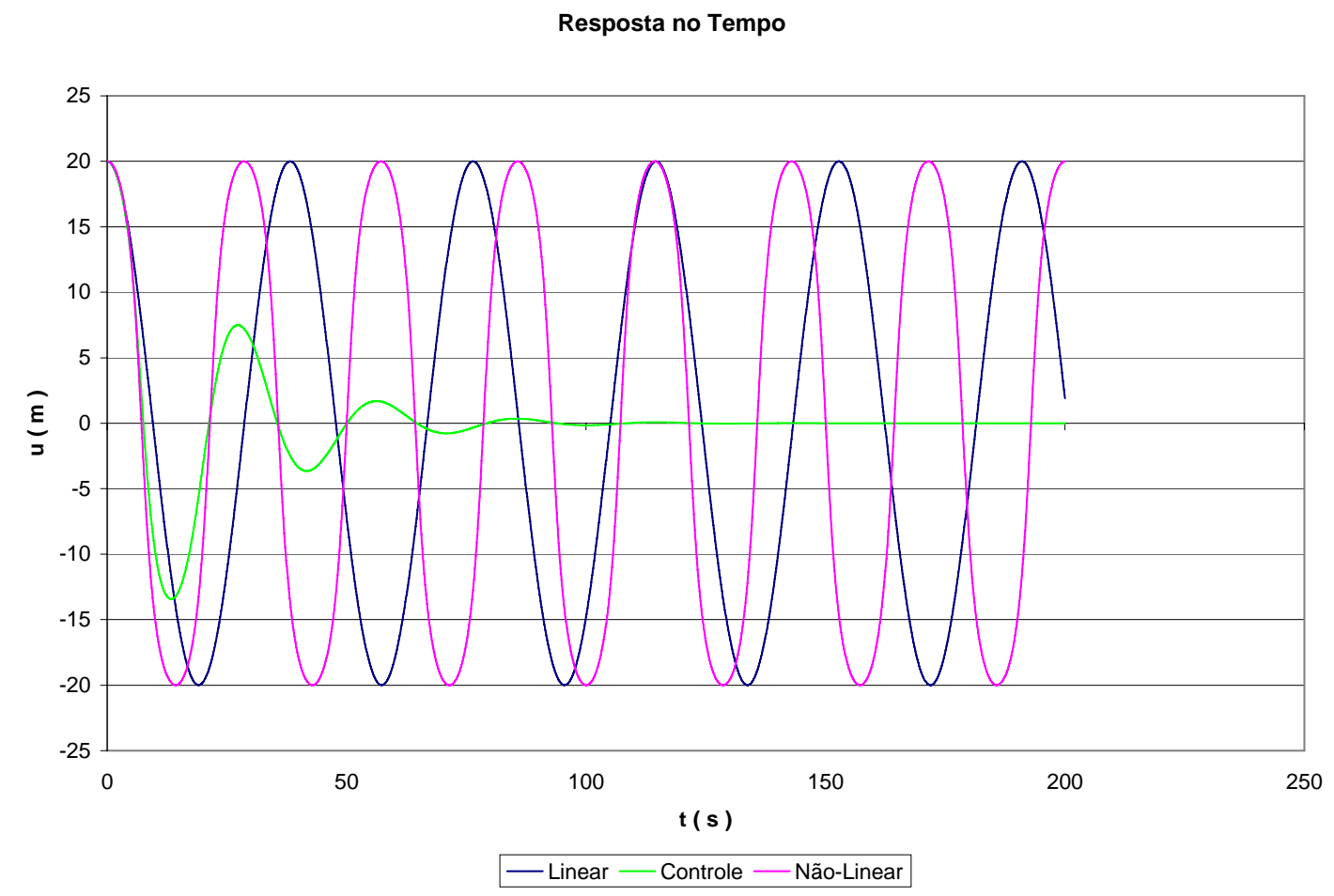

Figura 5.19 - "Riser" reto sem peso próprio com controle ótimo $Q=0.0005$ I e $Q_{o b}=0.0006 I$.

Primeiro modo; amplitude inicial de $20 \mathrm{~m}$.

Períodos: 30,23 s (linear); 28,58 s (não-linear); 29.08 s (controle).

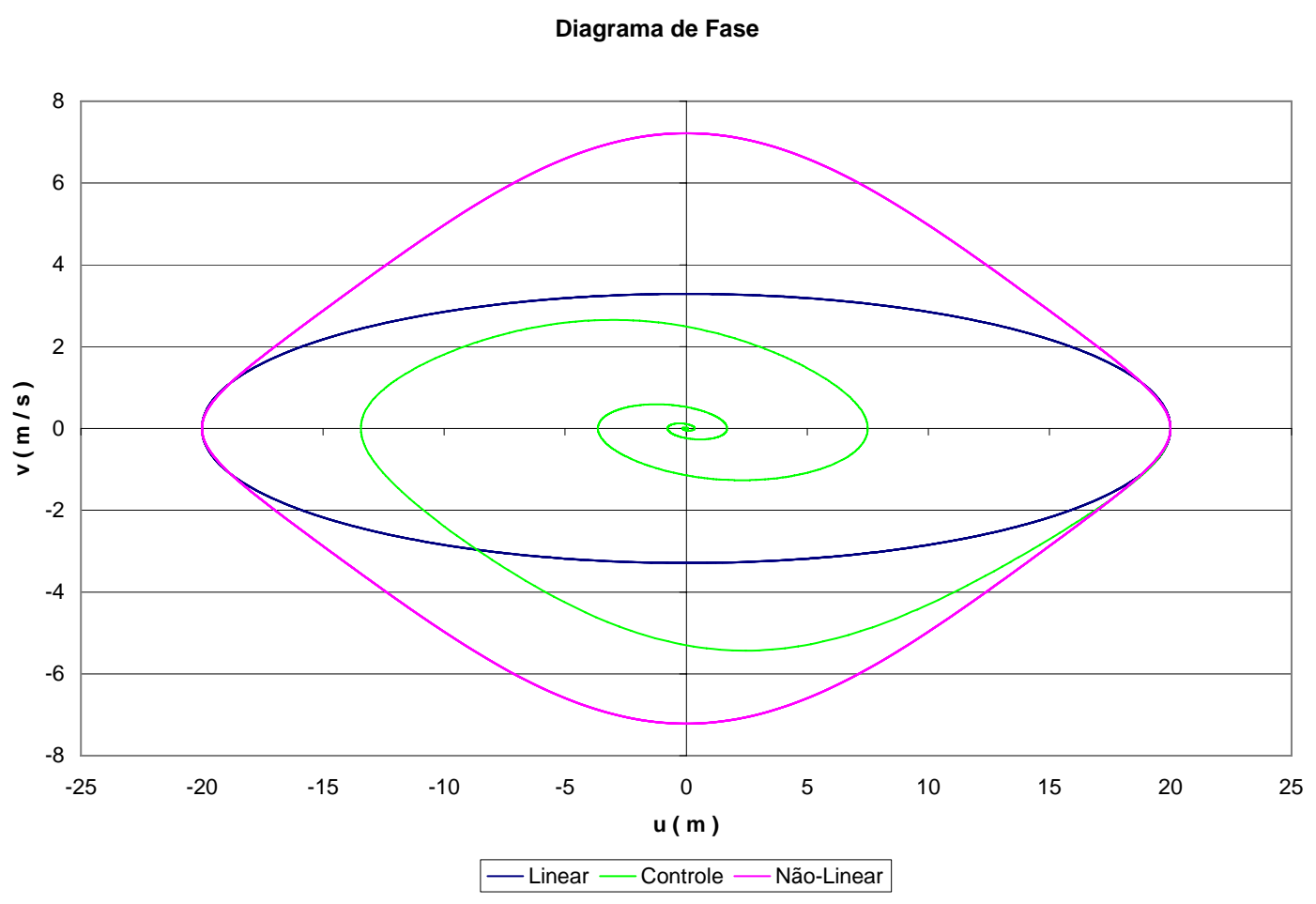

Figura 5.20 - Diagrama de fase ; primeiro modo; amplitude inicial de $20 \mathrm{~m}$. 
Força

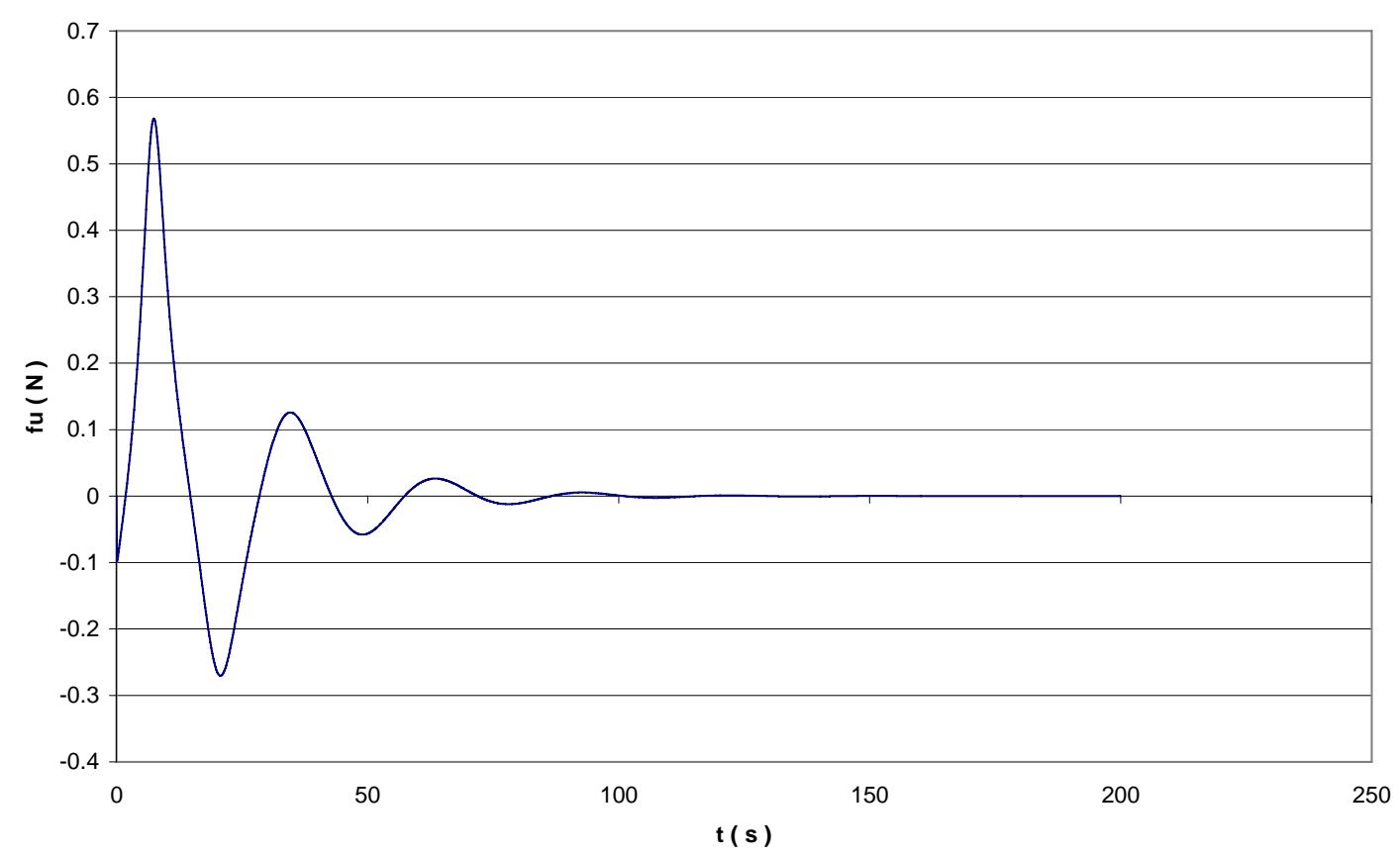

Figura 5.21 - Força de controle ; primeiro modo; amplitude inicial de $20 \mathrm{~m}$.

\subsection{2 “Riser” Reto com Peso Próprio}

Esta seção analisa os efeitos da introdução de um sistema de controle ativo ótimo para o riser reto com peso próprio do capítulo 3. Para tanto, admite-se $Q=0,0003 I, Q_{o b}=0,0004 I$ e $R=I$, onde I é a matriz identidade. A força modal do atuador $f_{u}$ é introduzida na equação não-linear do oscilador modal, conforme 5.36. Neste trabalho não será abordada a execução física do sistema de controle. A equação do oscilador modal não-linear com a força de controle é escrita na forma:

$$
\ddot{U}+2,70 \times 10^{-2} U+2,52 \times 10^{-4} U^{3}-2,27 \times 10^{-2} U V^{2}=f u .
$$

O sistema de controle ótimo representa um aumento na protensão de $\Delta T_{0}=23,65 t f$ ou $11,83 \%$. Esse sistema de controle leva, também, a uma taxa de amortecimento de $\zeta=0,3107$. Tal alteração da força de protensão influencia a freqüência aumentando-a. Nota-se que o período do sistema com controle ótimo $(36,43 s)$ é menor do que a do sistema não-linear $(39,36 s)$, conforme figura 5.22. 


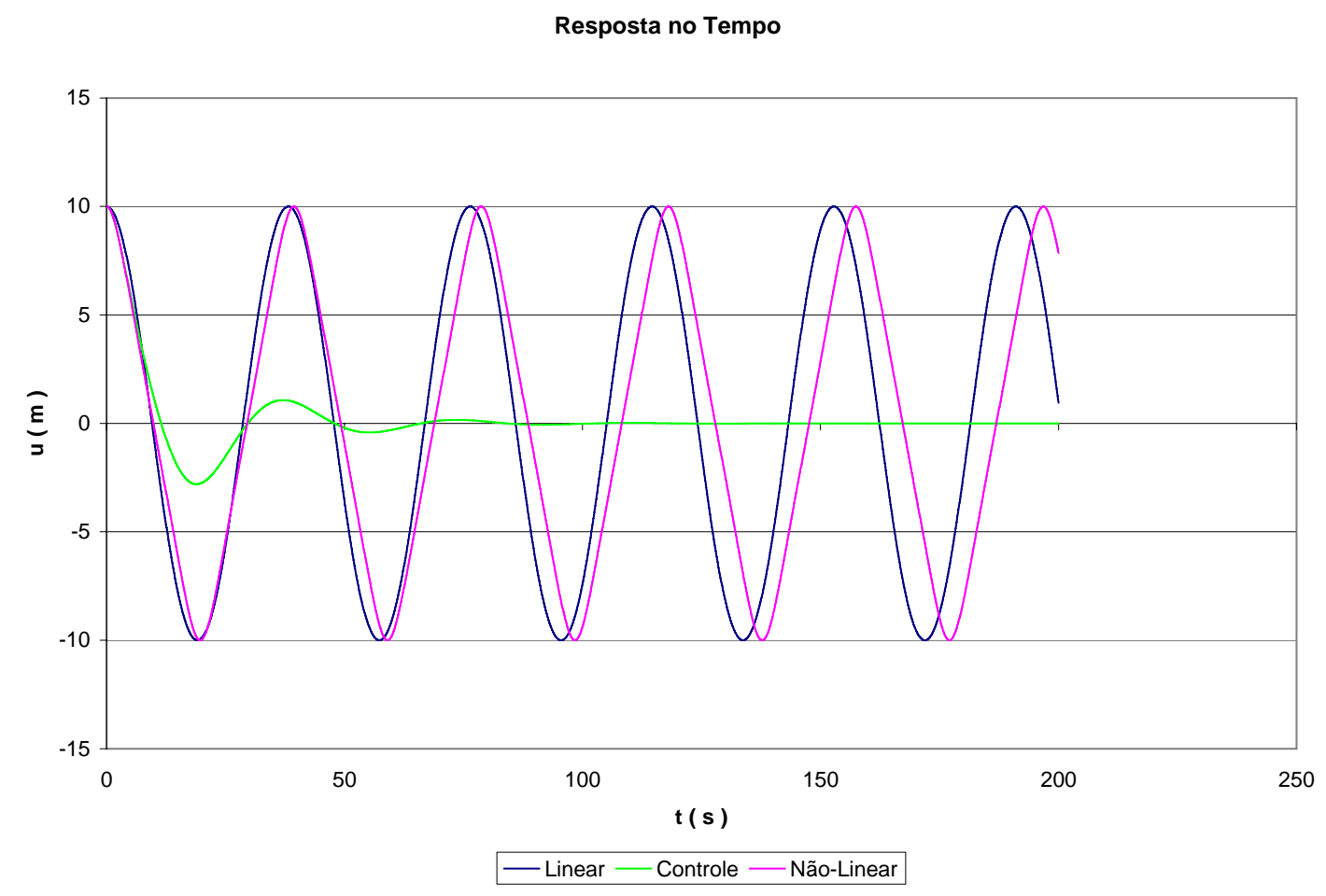

Figura 5.22 - "Riser" reto com peso próprio com controle ótimo $Q=0.0003 \mathrm{I}$ e $Q_{o b}=0.0004 I$.

Primeiro modo; amplitude inicial de $10 \mathrm{~m}$.

Períodos: 30,23 s (linear); 39,36 s (não-linear); 36,43 s (controle).

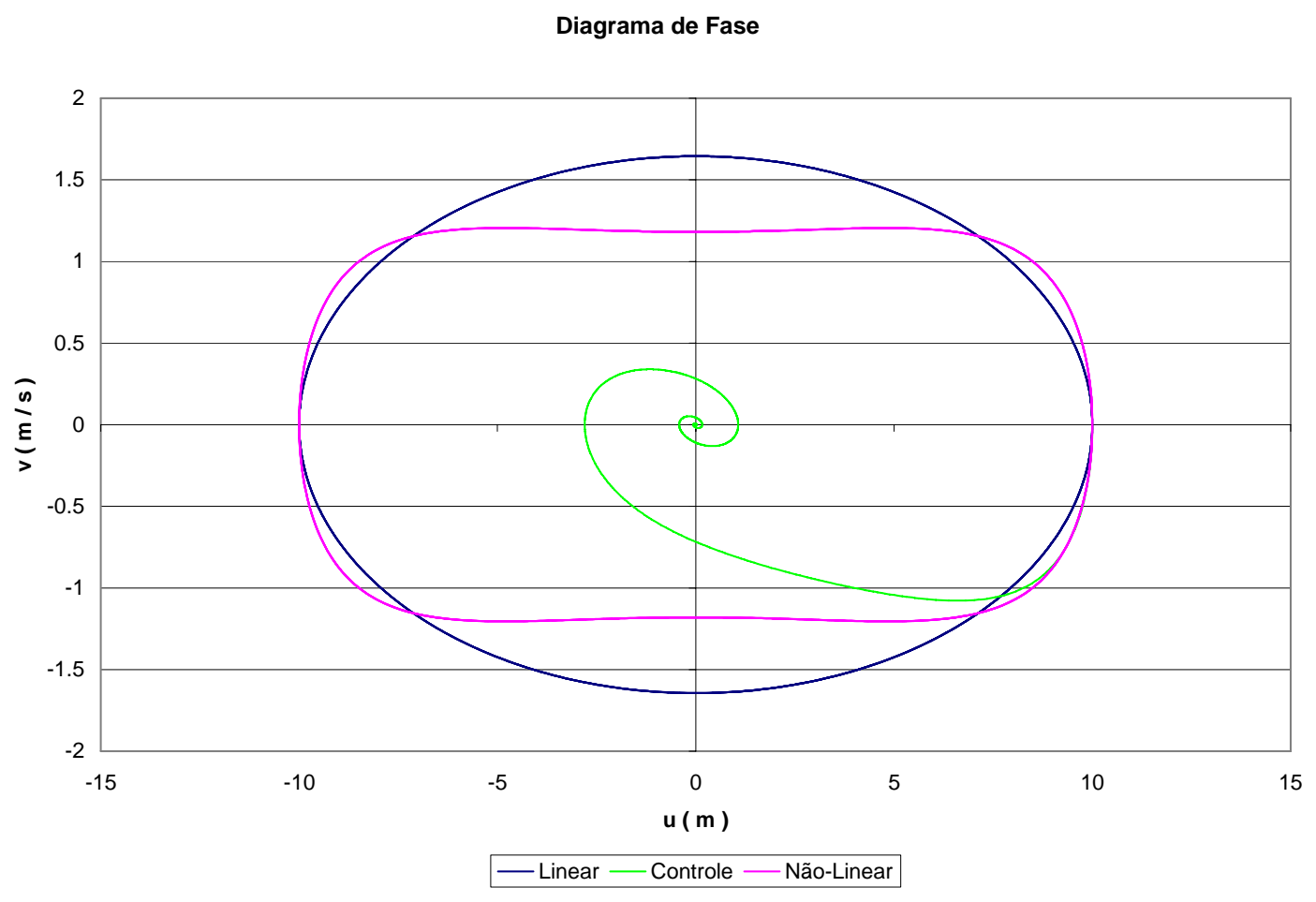

Figura 5.23 - Diagrama de fase; primeiro modo; amplitude inicial de $10 \mathrm{~m}$. 
Força

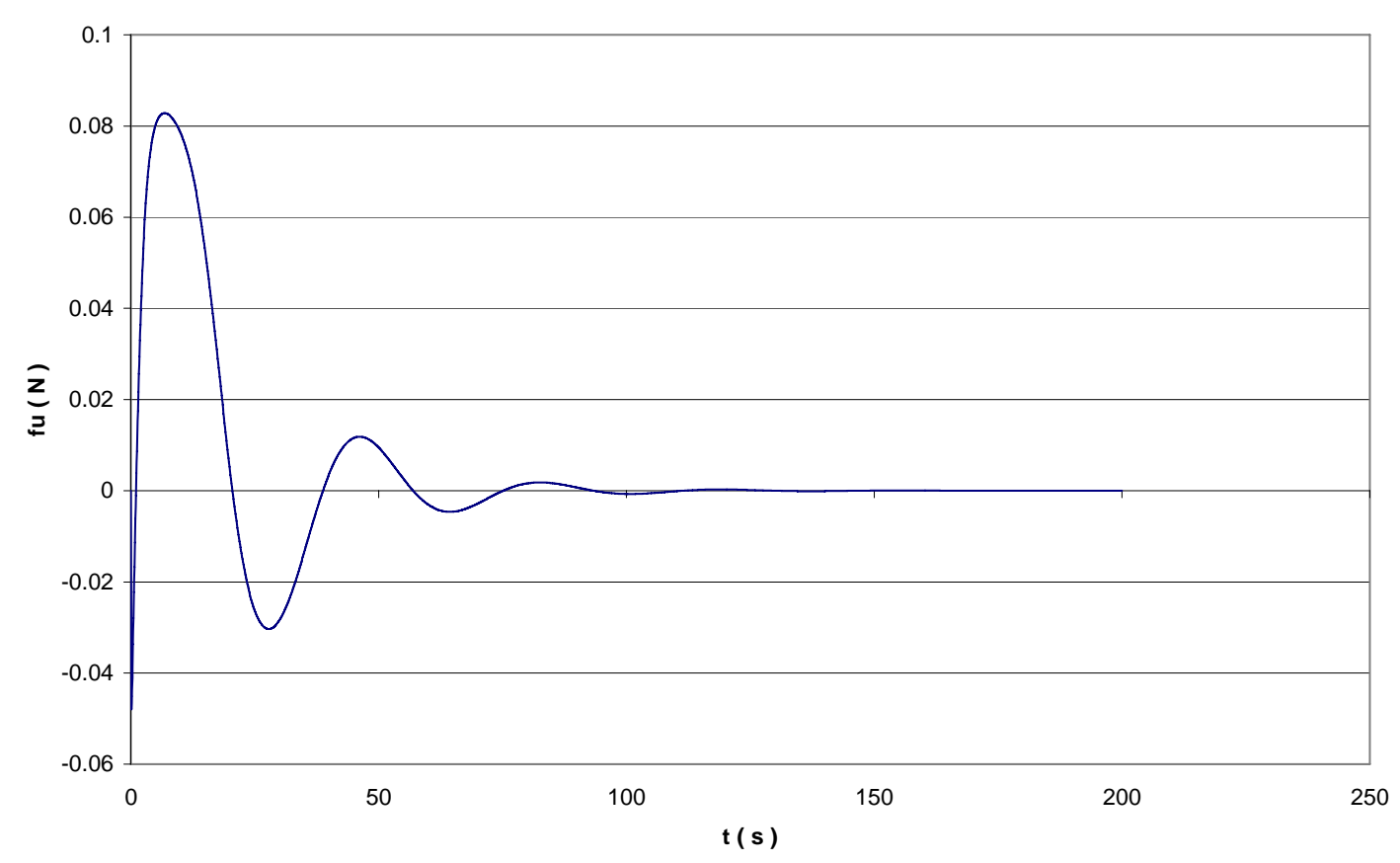

Figura 5.24 - Força de controle ; primeiro modo; amplitude inicial de $10 \mathrm{~m}$.

No entanto, o período do sistema linear $(30,23 s)$ é o menor e, portanto, o de maior freqüência, ou seja, mesmo com o aumento da tensão inicial os efeitos nãolineares de flexibilização, oriundos do termo em $U V^{2}$, levam a uma redução da freqüência do sistema com controle ótimo em relação ao sistema linear. O mesmo sistema estrutural, porém com diferente sistema de controle, apresentado na seção 5.2 leva a um período ligeiramente superior $(38,09 s)$. Esta variação do período está associada ao aumento na tensão inicial que leva a uma redução das amplitudes. Comparando-se as figuras 5.8 e 5.22, encontram-se as primeiras amplitudes negativas $4,01 \mathrm{~m}$ e $2.80 \mathrm{~m}$, respectivamente. Isto significa uma redução de 30,17\%. Comparandose as figuras 5.11 e 5.25 , encontram-se as primeiras amplitudes negativas 4,42m e $3.04 m$, respectivamente, portanto, uma redução de 31,22\%. 


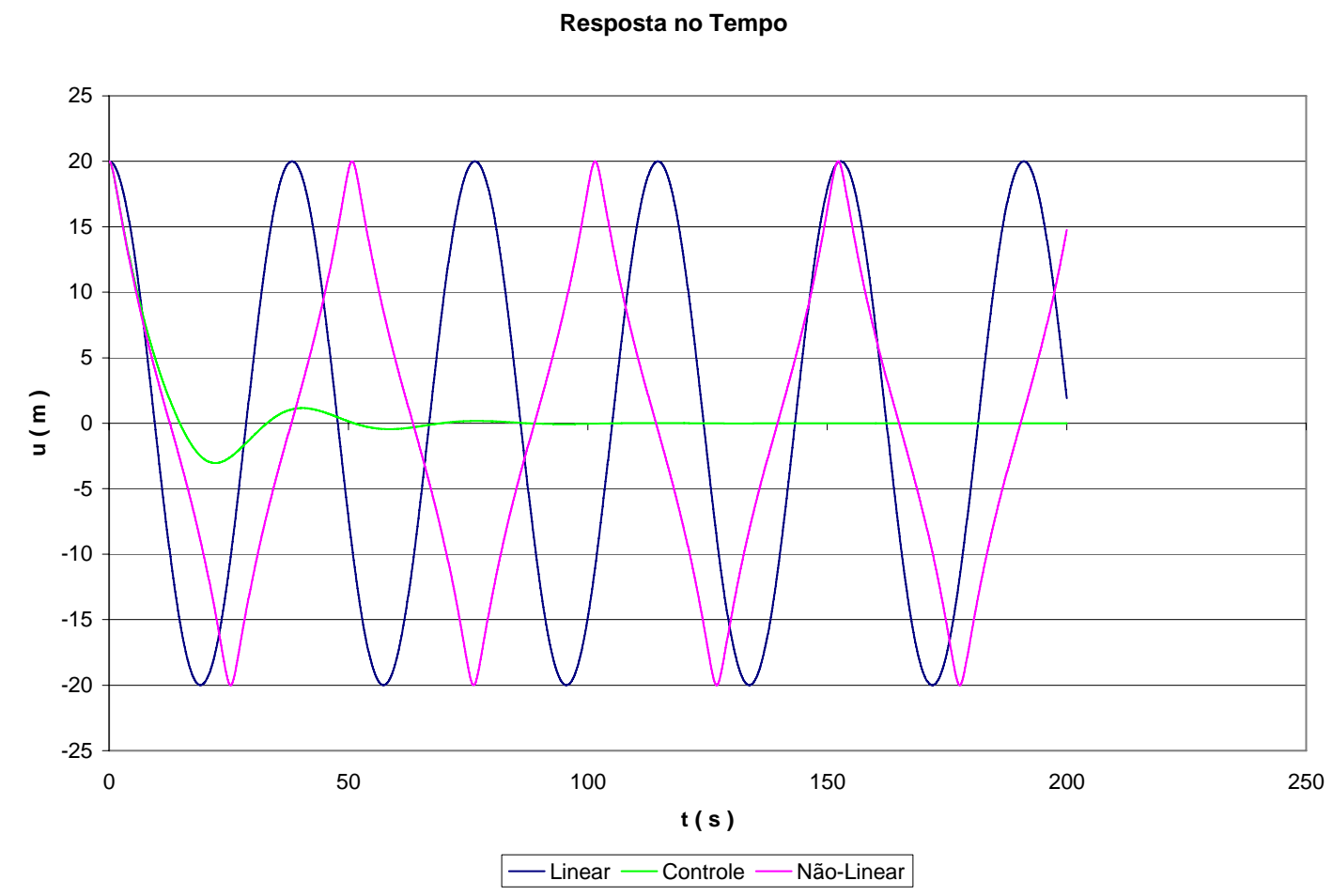

Figura 5.25 - "Riser" reto com peso próprio com controle ótimo $Q=0.0003 \mathrm{I}$ e $Q_{o b}=0.0004 \mathrm{I}$.

Primeiro modo; amplitude inicial de $20 \mathrm{~m}$.

Períodos: 30,23 s (linear); 50,77 s (não-linear); 36,67 s (controle).

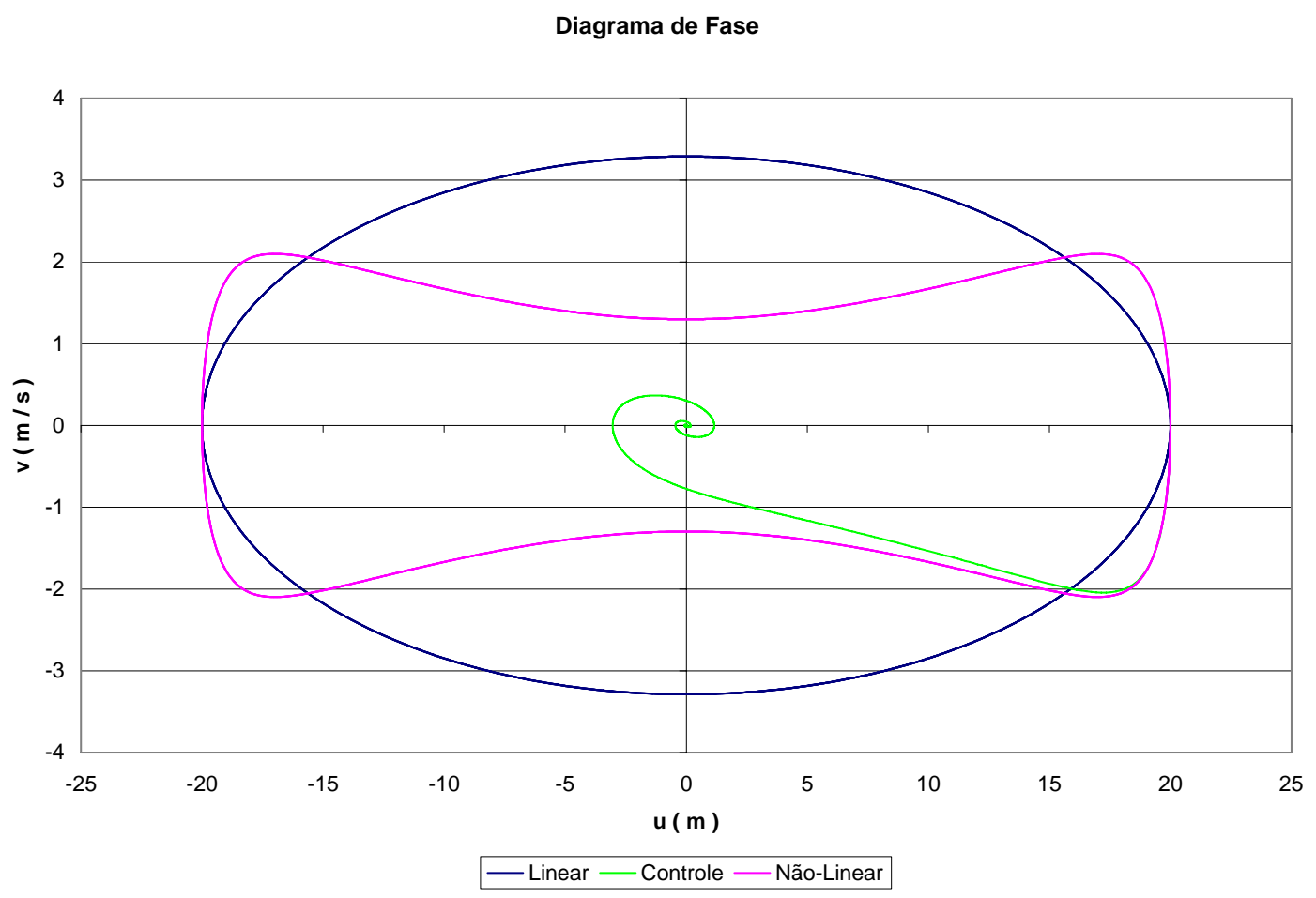

Figura 5.26 - Diagrama de fase; primeiro modo; amplitude inicial de $20 \mathrm{~m}$. 
Força

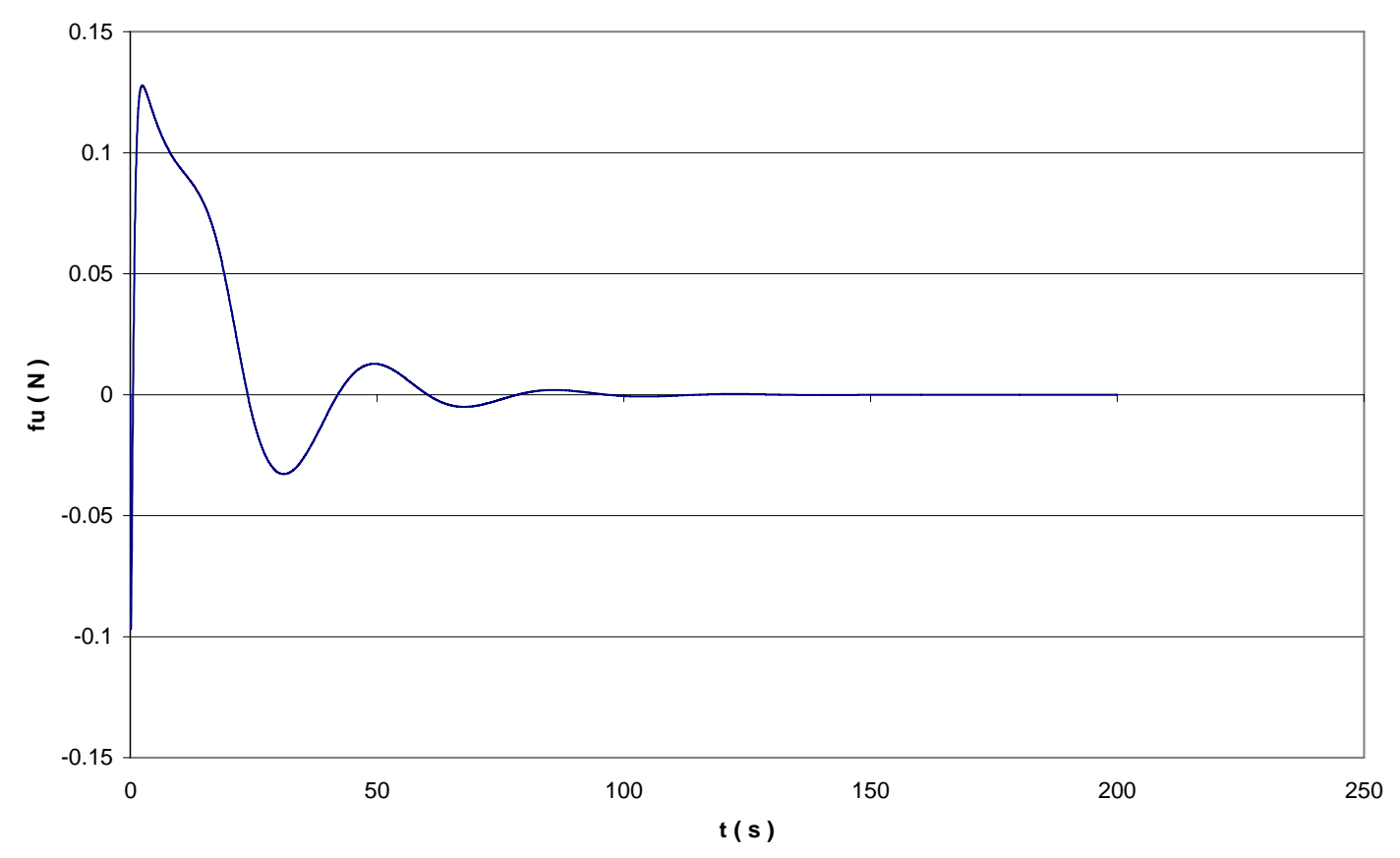

Figura 5.27 - Força de controle; primeiro modo; amplitude inicial de $20 \mathrm{~m}$.

A figura 5.24 mostra a resposta da força modal $u$ no tempo para a amplitude inicial de $10 \mathrm{~m}$ e a figura 5.27 para amplitude inicial de $20 \mathrm{~m}$. A força $f_{u}$ inicia com valor nulo $\left(f_{u}=0\right.$ para $\left.t=0\right)$. Mais uma vez, o sistema de controle ótimo se mostra estável. Observando as figuras 5.23 e 5.26, nota-se a tendência não-linear inicial do sistema com controle, que é bastante marcada na figura 5.26. Nessa mesma figura vale notar o acentuado estrangulamento do diagrama de fase no intervalo com deslocamentos próximos de zero. Este feito se deve ao termo em $U V^{2}$ e mostra a flexibilização do sistema, uma vez que a trajetória no diagrama de fase é maior e, portanto, o período do sistema é maior também.

Por fim, o estudo dos sistemas de controle se mostra valioso não só pela possibilidade do desenvolvimento de tais sistemas em aplicações práticas, mas, também, para a otimização e calibração dos sistemas já em operação, através do ajuste dos valores ótimos de tensão nos risers e dos valores de amortecimento pelo estudo de sistemas reduzidos contendo possivelmente apenas um ou dois modos não-lineares de vibração que representem adequadamente o comportamento do sistema estrutural em função da geometria e tipo de excitação que se deseja estudar. 


\subsubsection{Riser Reto com VIV e Controle}

Para o riser abordado na seção 3.3.1, estuda-se, nesta seção, seu comportamento dinâmico quando consideradas vibrações induzidas por vórtices (VIV) e controle. O sistema de controle adotado é o mesmo apresentado na seção anterior. As equações que representam a dinâmica do fluido foram retiradas de Blevins [10] e Parra [67]. Admite-se que, para o seguinte número de Strouhal $S_{t}=\frac{f D}{U} \cong 0,20,0$ primeiro modo seja excitado.

Sendo assim, a figura 5.28 mostra a resposta no tempo para o deslocamento do grau de liberdade posicionado na metade do comprimento do riser. Nota-se um regime transiente inicial seguido do regime permanente. Apesar de a amplitude do sistema não-linear não ser muito diferente da amplitude do sistema linear, é possível observar uma redução da amplitude máxima em módulo de 2,0 m, encontrada na seção 3.3.1, para 1,9 m, encontrada nessa seção. Tal redução se deve à elevação de 11.83\% na força de protensão. Novamente, a tensão do riser mostra-se pouca adequada para ser utilizada como variável de controle. A elevação da tensão reduz pouco a amplitude das vibrações, indicando a baixa controlabilidade do sistema. Os resultados aqui apresentados sobre a dinâmica de risers excitados por VIV e com sistema de controle, não são conclusivos. Ao contrário, estes resultados têm o intuito de, apenas, mostrar os possíveis problemas e potencialidades da abordagem por modos não-lineares na representação adequada da dinâmica de risers. Também, objetiva-se a compreensação da dinâmica não-linear dos risers com e sem um sistema de controle, estudando-se, por exemplo, os efeitos da mudança da tensão e apontando novas idéias como a utilização de massas móveis ao longo das linhas para a atenuação das vibrações. 


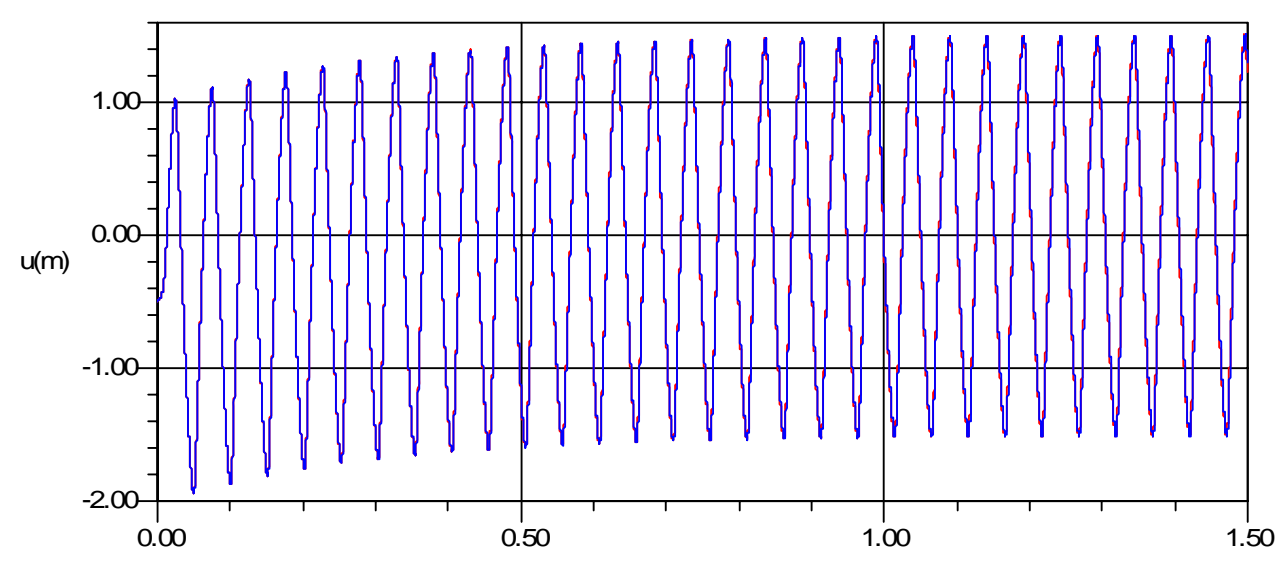

$0.00<\mathrm{X}<1.50 \mathrm{e}+3 ;-1.9<\mathrm{Y}<1.5 \quad \mathrm{t}(\mathrm{s})(103)$

Figura 5.28 - Resposta no tempo, riser reto com controle - Linear (Azul) e Não Linear (Vermelho).

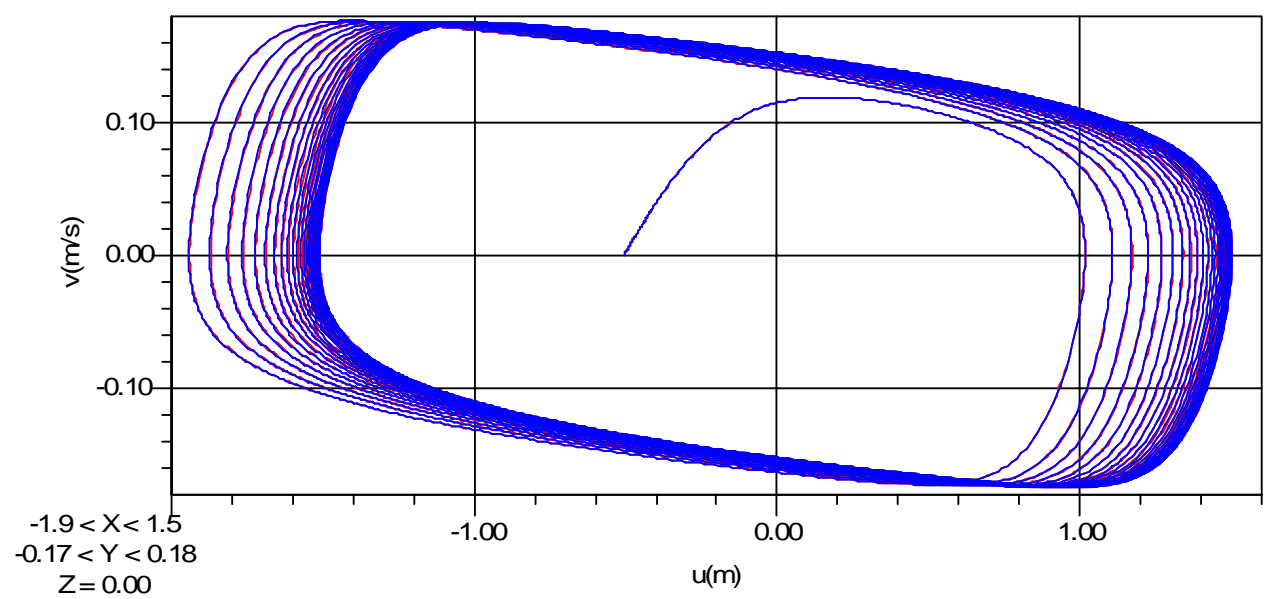

Figura 5.29 - Diagrama de fase, riser reto com controle - Linear (Azul) e Não Linear (Vermelho). 


\section{Conclusão}

Neste trabalho foram abordados modos normais não-lineares de vibração e multi-modos em risers discretizados pelo método dos elementos finitos. Além disso, foi estudada a dinâmica desses sistemas estruturais com a introdução de dispositivos de controle ativo. Os risers foram discretizados através do método dos elementos finitos. Tal abordagem foi bastante eficiente, uma vez que o método dos elementos finitos se mostra adequado para abordar sistemas estruturais com geometrias e propriedades físicas complexas.

Modos não-lineares de vibração são uma extensão natural do conceito de modos de vibração para sistemas lineares. Diferentemente dos modos de vibração clássicos, os modos não-lineares apresentam uma variação de sua forma modal e de sua freqüência em função da amplitude do movimento. Desta forma, um único modo não-linear apresenta conteúdos de diferentes freqüências associadas a diferentes modos lineares. Multi-modos são entendidos como uma extensão dos modos nãolineares normais de vibração no caso em que há acoplamento não-linear entre dois ou mais dos correspondentes modos lineares. Tais interações se devem à existência de ressonância interna. A compreensão deste fenômeno é importante para a elaboração de modelos para análise de VIV.

Foram estudados, primeiramente, os modos normais de risers retos sem peso próprio, com peso próprio e com carregamento distribuído induzido por corrente. Também foram estudados os multi-modos não-lineares de vibração sem peso próprio e com peso próprio. Duas abordagens distintas foram utilizadas: uma analítica e outra numérica. Para a primeira, as equações de movimento, os modos normais e os multimodos foram determinados analiticamente. Depois, para o mesmo sistema estrutural, determinaram-se as equações de movimento, os modos normais e os multi-modos utilizando-se um sistema discretizado pelo método dos elementos finitos. As respostas das duas abordagens (analítica e MEF) foram comparadas. Para um determinado intervalo de amplitude, as duas abordagens mostraram uma boa aderência no caso sem peso próprio. Porém, para valores altos de amplitudes, encontraram-se divergências entre as duas metodologias para todos os exemplos. Tal comportamento é coerente 
uma vez que a abordagem analítica se vale de simplificações mais drásticas do que as adotadas na via numérica. As simplificações adotadas na solução analítica fazem com que a forma modal não-linear analítica seja similar à forma modal linear, o que não se verifica para a solução numérica, e, portanto, as soluções analítica e numérica possuem topologias distintas. Além disso, a solução analítica não consegue capturar os efeitos inerciais, caracterizados pelos termos em $U V^{2}$, que representam o fenômeno das travelling waves.

Notou-se a capacidade de os modos não-lineares capturarem efeitos de softening e hardening, o que não seria possível explicar através dos modos lineares. Notaram-se variações da freqüência com a amplitude e variações nas trajetórias no diagrama de fase. Ficou claro, também, o potencial dos multi-modos para abordar ressonâncias internas que, por exemplo, poderiam ser excitadas por vibrações induzidas por vórtices.

Para os SCR's, foram determinados os modos não-lineares normais, via abordagem numérica, ou seja, utilizando-se o método dos elementos finitos. A abordagem analítica não foi utilizada devido ao elevado esforço matemático envolvido em função, especialmente, da geometria complexa dos SCR's. No entanto, novas abordagens analíticas têm obtido sucesso na modelagem de SCR's, ver [52]. Duas condições de contorno foram utilizadas: fixo-fixo e fixo-ancorado. Observou-se nestes exemplos que mesmo um único modo normal não-linear é capaz de capturar a influência de modos superiores na sua forma modal e na sua trajetória no diagrama de fase. Efeitos de softening e hardening foram encontrados. Além disso, notou-se a assimetria das amplitudes modais devido à geometria do riser, o que não seria possível apreciar utilizando-se apenas os modos lineares. Notou-se, também, a influência do sistema de amarração que absorve parte da energia do sistema, reduzindo os valores das amplitudes.

Não obstante, sistemas de controle ativo foram introduzidos nos risers retos sem e com peso próprio. Também foram estudados sistemas de controle ótimo. Nestes exemplos ficou evidente a eficiência dos modos não-lineares em capturar a dinâmica global dos risers com poucos graus de liberdade. Os sistemas de controle foram projetados utilizando apenas um único modo não-linear. Para trabalhos futuros, seria 
interessante utilizar mais de um modo não-linear, pois ao controlar apenas um modo de vibração outros modos podem ser excitados, por exemplo, através de ressonância interna. Nestes estudos, mostrou-se a possibilidade de desenvolver sistemas de controle ativo que, possivelmente, reduziriam as amplitudes de vibração dos risers, aumentando vida à fadiga dessas estruturas. No entanto, a utilização da tensão do riser como variável de controle mostrou-se pouco eficiente levando a grandes aumentos de tensão para pequenos decréscimos da amplitude do movimento. Tal comportamento deixa em evidência a baixa controlabilidade do sistema.

O estudo da dinâmica dos risers excitados por VIV (vibrações induzidas por vórtices) mostrou a capacidade dos modos não-lineares de, através de poucos modos, capturarem a essência do comportamento do sistema, como, por exemplo, no caso de $S C R$, o modelo foi capaz de capturar os efeitos da assimetria geométrica. Embora as amplitudes totais dos modelos linear e não-linear sejam próximas, a assimetria geométrica leva ao deslocamento do valor médio dos deslocamentos e, portanto, das tensões também. Este efeito pode alterar significativamente o cáculo da vida à fadiga. Estes modelos mostram-se úteis, também, no estudo de sistema de controle. Por serem modelos menores, consomem menos tempo de simulação, mesmo sendo modelos nãolineares, levando a respostas mais rápidas, essenciais para sistemas de controle eficientes. $\mathrm{Na}$ verdade, a maior parte do tempo de processamento é gasta na determinação dos modos não-lineares.

A abordagem via modos não-lineares de vibração mostra-se, portanto, capaz de reduzir o número de graus de liberdade dos sistemas estruturais sem perder o comportamento, ou característica, principal dos sistemas, sendo necessários poucos modos para representar adequadamente o comportamento global dos risers. Isto se deve à capacidade de um único modo não-linear capturar a influência de modos superiores devido à consideração de termos não-lineares na sua formulação. Assim um único modo não-linear pode guardar informações de dois ou mais modos lineares.

Por fim, apontam-se alguns trabalhos futuros. O primeiro deles seria, utilizando multi-modos, estudar as ressonâncias internas geradas pelas vibrações induzidas por vórtices. Para tanto seria necessário acoplar às equações não-lineares de movimentos as equações não-lineares do fluido. Isto poderia ser feito acoplando um 
oscilador de Van der Pol a cada nó do modelo de elementos finitos e fazendo a projeção do vetor de carregamentos nos modos não-lineares selecionados para estudo. O trabalho desenvolvido por Silveira et al [83] poderia ser de grande valia para este fim. Um outro desafio seria a modelagem e implementação computacional do TDZ (Touch Down Zone) para uma abordagem via modos não-lineares. Talvez seja necessária a consideração de elementos de contato, ou, o que seria ainda mais desafiador, uma mudança de variáveis, que transforme o problema de contornos móveis em contornos fixos, ver [52]. 


\section{Referências Bibliográficas}

[1] Achkire, Y., Preumont, A., Active tendon control of cable-stayed bridges, Earthquake Engineering and Structural Dynamics, vol. 25(6), pp. 585-597, 1996.

[2] Achkire, Y., Preumont, A., Optical measurement of cable and string vibration, Shock and Vibration, vol. 5, pp. 171-179, 1998.

[3] Aranha, J.A.P., Weak three dimensionality of a flow around a slender cylindder : the Ginzburg-Landau equation, Journal of the Brazilian Society of Mechanical Sciences and Engineering, Rio de Janeiro, v. 26, n. 4, p.355-367, October-December, 2004.

[4] Aranha, J.A.P., The flow around a slender cylinder: the Ginzburg-Landau equation, Conference on Bluff Body Wakes and Vortex-Induced Vibrations: Proceedings of BBVIV, 4, 1, Santorini, 2005.

[5] Baracho Neto, O.G.P., Mazzilli, C.E.N., Evaluation of non-linear normal modes for finite-element models, Computers and Structures, vol. 80, pp. 957-965, 2002.

[6] Baracho Neto, O.G.P., Modos normais e multi-modos na dinâmica das estruturas de comportamento não-linear, Tese de Doutorado, Escola Politécnica da Universidade de São Paulo, 2003.

[7] Baracho Neto, O.G.P., Mazzilli, C.E.N., Evaluation of multi-modes for finiteelement models: systems tuned into 1:2 internal resonance, International Journal of Solids and Structures, vol. 42, pp. 5795-5820, 2005.

[8] Baracho Neto, O.G.P., Mazzilli, C.E.N., Evaluation of multi-modes for finiteelement models: systems tuned into 1:3 internal resonance, to be published in Journal of Sound and Vibration, 2008.

[9] Bender, C.; Orszag, S., Advanced Mathematical Methods for Scientists and Engineers, McGraw-Hill, 1978.

[10] Blevins, R.D., Flow-Induced Vibration, Second Edition, Krieger, 2001.

[11] Boivin, N.; Pierre, C.; Shaw, S.W.; Non-linear modal analysis of structural systems using multi-mode invariant manifolds, AIAA Dynamics Specialists Conference, South Carolina, 1994.

[12] Boivin, N.; Pierre, C.; Shaw, S.W.; Non-linear modal analysis of structural systems featuring internal resonances, Journal of Sound and Vibration, 182 (2), pp. 336-341, 1995.

[13] Boivin, N.; Pierre, C.; Shaw, S.W.; Non-linear modal analysis of the forced response of structural systems, AIAA Dynamics Specialists Conference, Utah, 1996. 
[14] Bossens, F. \& Preumont, A., Active tendon control of cable-stayed bridges: a large-scale demonstration, Earthquake Engineering and Structural Dynamics, vol. 30, pp. 961-979, 2001.

[15] Brasil, R.M.L.R.F., Mazzilli, C.E.N., A general formulation of non-linear dynamics applied to accessing the static loading effect upon the dynamic response of planar frames, Applied Mechanics Review, vol 46 (11), pp.110-117, 1993.

[16] Braun M., On some properties of the multiple pendulum, Archive of Applied Mechanics, 72, 899-910, 2003.

[17] Chang, S. M., Pulse response based minimum time control of flexible structures, Tese de Doutorado, University of Texas at Austin, 1993.

[18] Chirikov, V.A., Causes for the softening phenomenon at vibrations of beams undergoing large rotation, ENOC-2005, Eindhoven, Netherlands, 2541-2547, 2005.

[19] Clough, R.W., Penzien, J., Dynamics of Structures, McGraw-Hill, 1975.

[20] Elsgolts, L. E., Differential equations and the calculus of variations, Moscow, Mir, 1970.

[21] Fujino, Y., Susumpow, T., An experimental study on active control of planar cable vibration by axial support motion, Earthquake Engineering and Structural Dynamics, vol. 23, pp. 1283-1297, 1994.

[22] Fujino, Y., Warnitchai, P., Pacheco, M., Active stiffness control of cable vibration, ASME Journal of Applied Mechanics, vol. 60, pp. 948-953, 1993.

[23] Fung, Y.C., An Introduction to the Theory of Aeroelasticity, Dover, 1969.

[24] Gattulli, V., Pasca, M., Vestroni, F., Nonlinear oscillations of a nonresonant cable under in-plane excitation with a longitudinal control, Non-Linear Dynamics, vol. 14, pp. 139-156, 1997.

[25] Gattulli, V., Paolone, A., Planar motion of a cable supported beam with feedback controlled actions, Journal of Intelligent Materials Systems and Structures, vol. 8, pp. 767-774, 1997.

[26] Gattulli, V., Romeo, F., Integrated procedure for identification and control of MDOF structures, Journal of Engineering Mechanics, vol. 126 (7), pp. 730-737, 2000.

[27] Gattulli, V., Lepidi, M., Non-Linear interactions in the planar dynamics of cablestayed beam, International Journal of Solids and Structures, vol. 40, pp. 4729-4748, 2003.

[28] Gattulli, V., Lepidi, M., Macdonald, J. \& Taylor, C., One-to-two global-local interaction in a cable-stayed beam observed through analytical, finite element and 
experimental models, International Journal of Non-Linear Mechanics, vol. 40, Issue 4, pp. 571-588, May 2005.

[29] Gendelman, O., Manevitch, L.I., Vakakis, A.F., Bergman, L., A degenerate bifurcation structure in the dynamics of coupled oscillators with essential stiffness nonlinearities, Nonlinear Dynamics, vol. 33 (1), pp. 1-10, 2003.

[30] González Lima, R., Experimental investigation of minimum time translation of a flexible beam using pulse response based control, Tese de Doutorado, University of Texas, 1996.

[31] Hirsch, M.W., Smale, S., Differential Equations, Dynamical Systems and Linear Álgebra, Academic Press, 1974.

[32] Irvine, H.M., Caughey, T.K., The linear theory of the free vibrations of a suspended cable, Proceedings of the Royal Society of London, A. vol. 341, pp 299315, 1974.

[33] Jurjo, D.L.B.R., Vibration analysis of slender columns under self-weight using digital image processing techniques, EUROMECH, Colloquium 483 - Geometrically Non-linear Vibrations of Structures, 9 - 11 July 2007, FEUP, Porto, Portugal.

[34] Kauderer, H., Nichtlineare Mechanik, Springer-Verlag, 1958.

[35] Keber, M., Wiercigroch, M., Sanches, C.T., Mazzilli, C.E.N., Application of nonlinear normal modes to vortex induced vibration of risers, Euromech 484, Delft, 2006.

[36] Keber, M., Wiercigroch, M., Analysis of a vertical offshore riser with weak geometrical nonlinearity excited by currents, Euromech 483, Porto, 2007.

[37] Keber, M., Wiercigroch, M., Dynamics of a vertical riser with weak structural nonlinearity excited by wakes, Journal of Sound and Vibration, vol. 315, Issue 3, pp. 685-699, 19 August 2008.

[38] Keber, M., Wiercigroch, M., A reduced order for vortex-induced vibration of vertical riser in lock-in, Proceedings of the IUTAM Symposium on Fluid- Structure Interaction in Ocean Engineering, Hamburg, pp. 155-166, July 2007.

[39] King, M.E., Vakakis, A.F., An energy-based approach to computing resonant nonlinear normal modes, Journal of Applied Mechanics-Transactions of the ASME, vol. 63 (3), pp. 810-819, 1996.

[40] Kuiper, G., Metrikine, A., Efthymiou, M., Experimental investigation of the dynamic behaviour of a water intake riser, OMAE 2007, San Diego, 2007.

[41] Lacarbonara, W., Rega, G., Nayfeh, A. H., Resonant non-linear normal modes. Part I: analytical treatment for structural one-dimensional systems, International Journal of Non-Linear Mechanics, vol. 38, pp. 851-872, 2003. 
[42] Lacarbonara, W., Rega, G., Resonant non-linear normal modes. Part II: activation/orthogonality conditions for shallow structural systems, International Journal of Non-Linear Mechanics, vol.38, pp.873-887, 2003.

[43] Love, AE.H., A Treatise on the Mathematical Theory of Elasticity, 4th edition., Dover, 1944.

[44] Luenberger, D.G., Introduction to Dynamic Systems, Wiley, 1979.

[45] Manevitch, L.I., The description of localised normalised modes in a chain of nonlinear coupled oscillators using complex variables, Nonlinear Dynamics, vol. 25 (1-3), pp. 95-109, 2001.

[46] Manevitch, L.I., Gendelman, O., Musienko, A.I., Vakakis, A.F., Bergman, L., Dynamic interaction of a semi-infinite linear chain of coupled oscillators with a strongly nonlinear end attachment, Physica D, vol. 178 (1-2), pp. 1-18, 2003.

[47] Martins, C.A., Amortecimento ativo para reduzir vibrações induzidas por vórtices em risers. Tese de Doutoramento, Escola Politécnica da Universidade de São Paulo, 1989.

[48] Mazzilli, C.E.N. Non-linear finite-element formulation in dynamics-II, Boletim Técnico da Escola Politécnica da Universidade de São Paulo, Departamento de Engenharia de Estruturas e Fundações, BT/PEF/9008, 1990.

[49] Mazzilli, C.E.N., Soares, M.E.S. \& Baracho Neto, O.G.P., Reduction of finiteelement models of planar frames using non-linear normal modes, International Journal of Solids and Structures, vol. 38, pp. 1993-2008, 2001.

[50] Mazzilli, C.E.N., Sanches, C.T., Baracho Neto, O.G.P., Wiercigroch, M., Keber, M., Non-linear modes of beams subjected to axial loads, Euromech 483, Porto, 2007.

[51] Mazzilli, C.E.N., Sanches, C.E.S., Baracho Neto, O.G.P., Keber, M., Wiercigroch, M., Nonlinear modal analysis for beams subjected to axial loads: analytical and finite element solution, International Journal of Non-Linear Mechanics, vol. 43 , pp. 551-561, 2008.

[52] Mazzilli, C.E.N. \& Lenci, S., Normal vibration modes of a slender beam on elastic foundation with unilateral contact, XXII ICTAM, 25-29 de Agosto, 2008, Adelaide, Australia.

[53] Mazzilli, C.E.N. \& Wiercigroch, M., Effect of linearly varying normal force upon the non-linear modal analysis of slender beams, ENOC, Saint Petersburg, RUSSIA, June 30 to July 4, 2008.

[54] Meirovitch, L., Methods of Analytical Dynamics, McGraw Hill, 1970.

[55] Meirovitch, L., Elements of vibration analysis, New York, McGraw-Hill, 1975. 
[56] Meneghini, J.R., Projetos de Pesquisa no Tópico Geração e Desprendimento de Vórtices no Escoamento ao Redor de Cilindros, Livre Docência, Escola Politécnica da Universidade de São Paulo, São Paulo, 2002.

[57] Mikhlin, Y.V., Matching of local expansions in the theory of nonlinear vibrations, Journal of Sound and Vibration, vol. 182 (4), pp. 577-588, 1995.

[58] Mikhlin, Y.V., Zhupiev, A.L., An application of the Ince algebraization to the stability of non-linear normal vibration modes, International Journal of Non-Linear Mechanics, vol. 32 (2), pp. 393-409, 1997.

[59] Mikhlin, Y.V., Morgunov, B.I., Normal vibrations in near-conservative selfexcited and viscoelastic nonlinear systems, Nonlinear Dynamics, vol. 25 (1-3), pp. 33 48, 2001.

[60] Nayfeh, A.H., Mook, D.T., Nonlinear Oscillations, Wiley, 1979.

[61] Nayfeh A.H, Nayfeh S.A., On nonlinear modes of continuous systems, Journal of Vibration and Acoustics; vol. 116, pp. 129-136, 1994.

[62] Nayfeh, A.H., Chin, C., \& Nayfeh, S.A., Nonlinear normal modes of a cantilever beam, Journal of Vibration and Acoustics, vol. 117 (4), pp. 477-481, 1995.

[63] Nayfeh, A.H., Nonlinear interactions: analytical, computational and experimental methods, Meccanica, vol. 35 (6), pp. 583-586, 2000.

[64] Newberry, B.L. \& Perkins, N.C., Analysis of resonant tangential response in submerged cables resulting from 1-to-1 internal resonance, ISOPE 1997, Honolulu, vol. II, pp. 157-163, 1997.

[65] Ogata, K., Discrete-Time Control Systems, Prentice Hall, 1995.

[66] Pak, C.H., Rosenberg R.M., On the existence of normal mode vibrations in nonlinear systems, Quarterly of Applied Mathematics, pp. 403-416, 1968.

[67] Parra, P.H.C.C., Modelo Semi-Empírico de Vibrações Induzidas por Vortex Shedding - Análise Teórica e Experimental, Dissertação de Mestrado, Escola Politécnica da Universidade de São Paulo, 1996.

[68] Pars, L.A, A Treatise on Analytical Dynamics, Heinemann, 1965.

[69] Pesce, C.P., Mecânica de Cabos e Tubos Submersos Lançados em Catenária: Uma Abordagem Analítica e Experimental, Tese de Livre Docência, Escola Politécnica da Universidade de São Paulo, 1997.

[70] Pesce, CP, Fujarra, A.L.C., Simos, A.N., Tannuri, E.A., Analytical and closed form solutions for deep water riser-like eigenvalue problem, ISOPE 1999, vol. 2, pp. 255-264, Goa, Índia, 1999. 
[71] Pesce, C.P., and Martins, C.A., Numerical computation of riser dynamics, numerical modelling in fluid-structure interaction, Advances in Fluid Mechanics Series, S. Chakrabarti ed., WIT, chap. 7, pp. 253-309, 2005.

[72] Pesce, C.P., Martins, C.A., Silveira, L., Riser-soil interaction: local dynamics at TDP and a discussion on the eigenvalue and the VIV problems, Journal of Offshore Mechanics and Arctic Engineering, vol. 128, pp. 39-55, 2006.

[73] Preumont , A., Vibration Control of Active Structures An Introduction, 2nd ed., Kluwer, 2002.

[74] Preumont, A. \& Achkire, Y., Active dumping of structures with guy cables, AIAA, Journal of Guidance, Control and Dynamics, vol. 20(2), pp. 320-326, 1997.

[75] Rosenberg, R.M., On nonlinear vibrations of systems with degrees of freedom, Advances in Applied Mechanics, vol. 9, pp. 155-242, 1996.

[76] Saint-Marcoux, J., Meeting the challenges of ultra deep water riser systems, INTSOK Pipeline and Riser Workshop, Houston, 2004.

[77] Sanches, C.T., Modos não-lineares de vibração em arcos discretizados pelo método dos elementos finitos, Dissertação de Mestrado, Escola Politécnica da Universidade de São Paulo, 2003.

[78] Sanches, C.T., Mazzilli, C.E.N., Cunha, L.D., Pesce, C.P., Non-linear modal analysis applied to riser dynamics, ISOPE 2007, Lisboa, 2007.

[79] Shaw, S.W., Pierre, C., Non-linear normal modes and invariant manifolds, Journal of Sound and Vibration, vol. 150 (1), pp. 170-173, 1991.

[80] Shaw, S.W., Pierre, C., Normal modes for non-linear vibratory systems, Journal of Structures, vol. 164 (1), pp. 85-124, 1993.

[81] Shaw, S.W., Pierre, C., Normal modes of vibration for nonlinear continuous systems, Journal of Sound and Vibration, vol. 169(3), pp. 319-347, 1994.

[82] Shaw, S.W.; Pierre, C., Pesheck, E., Modal analysis-based reduced-order models for nonlinear structures - an invariant manifold approach, The Shock and Vibration Digest, vol. 31, pp. 3-16, 1999.

[83] Silveira, L.M.Y., Martins, C.A., Aranha, J.A.P., A qualitative study of the response of the Ginzburg-Landau equations when coupled to the structural model of a submerged cable, In: Fifth Conference on Bluff Body Wakes and Vortex-Induced Vibrations, 2007, Costa do Sauípe, Book of Papers, 2007, v. Único, p. 75-78.

[84] Silveira, L.M.Y., Martins, C.A., Cunha, L.D., Pesce, C.P., An investigation on the effect of tension variation on VIV of risers, OMAE, San Diego, 2007. 
[85] Singh G., Sharma A.K., Rao G.V., Large-amplitude free vibrations of beams - a discussion on various formulations and assumptions, Journal of Sound and Vibration vol.142(1), pp. 77-85, 1990.

[86] Soares, M.E.S., Modos não-lineares em sistemas discretizados pelo método dos elementos finitos, Tese de Doutorado, Escola Politécnica da Universidade de São Paulo, 1998.

[87] Soares, M.E.S., Mazzilli, C.E.N., Nonlinear normal modes of planar frames discretised by the finite element method, Computers and Structures, vol. 77, pp. 485493, 2000.

[88] Srinil, N., Rega, G. \& Chucheepsakul, S., Two-to-one resonant multi-modal dynamics of horizontal/inclined cables. Part I: Theoretical formulation and model validation, Nonlinear Dynamics, vol. 48, pp. 231-252, 2007.

[89] Srinil, N., Rega, G., Two-to-one resonant multi-modal dynamics of horizontal/inclined cables. Part II: Internal resonance activation, reduced-order models and nonlinear normal modes, Nonlinear Dynamics, vol. 48, pp. 253-274, 2007.

[90] Srinil, N., Rega, G., The effects of kinematic condensation on internally resonant forced vibrations of shallow horizontal cables, International Journal of Non-Linear Mechanics, vol. 42, pp. 180-195, 2007.

[91] Terceiro, E., Utilização de massas deslizantes para controle ótimo das vibrações de um braço flexível rotativo : generalização, simulação e resultados, Dissertação de Mestrado, Engenharia Mecânica, Escola Politécnica da Universidade de São Paulo, São Paulo, 2002.

[92] Vakakis, A.F., Rand, R.H., Normal modes and global dynamics of a two-degreeof-freedom non-linear system - I. Low energies, International Journal of Non-Linear Mechanics, vol. 27 (5), pp. 861-874, 1992.

[93] Vakakis, A.F., Rand, R.H., Normal modes and global dynamics of a two-degreeof-freedom non-linear system - II. High energies, International Journal of Non-Linear Mechanics, vol. 27 (5), pp. 875-888, 1992.

[94] Weaver Jr., W., Timoshenko, S.P., Young, D.H., Vibration problems in engineering, New York, Wiley, 5th ed., 1990.

[95] Weisstein, Eric W., Double Pendulum, http://scienceworld.wolfram.com/physics/DoublePendulum.html, em 23/03/2009.

[96] Wells, D.A., Theory and Problems of Lagrangian Dynamics, New York, McGraw-Hill, pp. 13-14, 24, and 320-321, 1967. 